\title{
Population-level Eccentricity Distributions of Imaged Exoplanets and Brown Dwarf Companions: Dynamical Evidence for Distinct Formation Channels*
}

\author{
Brendan P. Bowler ${ }^{1}$ (i), Sarah C. Blunt ${ }^{2,3,5}$ (1), and Eric L. Nielsen ${ }^{4}$ (B) \\ ${ }^{1}$ Department of Astronomy, The University of Texas at Austin, Austin, TX 78712, USA; bpbowler@astro.as.utexas.edu \\ ${ }^{2}$ Department of Astronomy, California Institute of Technology, Pasadena, CA, USA \\ ${ }^{3}$ Center for Astrophysics|Harvard \& Smithsonian, Cambridge, MA, USA \\ ${ }^{4}$ Kavli Institute for Particle Astrophysics and Cosmology, Stanford University, Stanford, CA 94305, USA \\ Received 2019 July 13; revised 2019 November 22; accepted 2019 November 23; published 2020 January 23
}

\begin{abstract}
The orbital eccentricities of directly imaged exoplanets and brown dwarf companions provide clues about their formation and dynamical histories. We combine new high-contrast imaging observations of substellar companions obtained primarily with Keck/NIRC2 together with astrometry from the literature to test for differences in the population-level eccentricity distributions of 27 long-period giant planets and brown dwarf companions between 5 and 100 au using hierarchical Bayesian modeling. Orbit fits are performed in a uniform manner for companions with short orbital arcs; this typically results in broad constraints for individual eccentricity distributions, but together as an ensemble, these systems provide valuable insight into their collective underlying orbital patterns. The shape of the eccentricity distribution function for our full sample of substellar companions is approximately flat from $e=0-1$. When subdivided by companion mass and mass ratio, the underlying distributions for giant planets and brown dwarfs show significant differences. Low mass ratio companions preferentially have low eccentricities, similar to the orbital properties of warm Jupiters found with radial velocities and transits. We interpret this as evidence for in situ formation on largely undisturbed orbits within massive extended disks. Brown dwarf companions exhibit a broad peak at $e \approx 0.6-0.9$ with evidence for a dependence on orbital period. This closely resembles the orbital properties and period-eccentricity trends of wide (1-200 au) stellar binaries, suggesting that brown dwarfs in this separation range predominantly form in a similar fashion. We also report evidence that the "eccentricity dichotomy" observed at small separations extends to planets on wide orbits: the mean eccentricity for the multi-planet system HR 8799 is lower than for systems with single planets. In the future, larger samples and continued astrometric orbit monitoring will help establish whether these eccentricity distributions correlate with other parameters such as stellar host mass, multiplicity, and age.
\end{abstract}

Unified Astronomy Thesaurus concepts: Exoplanet formation (492); Brown dwarfs (185); Extrasolar gas giants (509)

\section{Introduction}

The orbital eccentricities of exoplanets directly trace their formation and dynamical histories. Planets are expected to form on circular coplanar orbits within protoplanetary disks, but can develop nonzero eccentricities through dynamical interactions with other planets (e.g., Rasio \& Ford 1996; Weidenschilling \& Marzari 1996; Ford \& Rasio 2008; Jurić \& Tremaine 2008; Dawson \& Murray-Clay 2013; Petrovich \& Tremaine 2016), secular Kozai-Lidov perturbations with a massive outer companion (e.g., Naoz 2016; Mustill et al. 2017), or planetdisk interactions (e.g., Goldreich \& Sari 2003). Over time, the eccentricities of close-in planets can be damped due to tidal dissipation with the host star (e.g., Ogilvie 2014) and at young ages as a result of torques and dynamical friction within a gas or planetesimal disk (Duffell \& Chiang 2015; Morbidelli 2018).

The observed eccentricities of giant planets measured from radial velocity surveys span the entire range of bound orbits $(0 \leqslant e<1)$, in stark contrast to the nearly circular orbits of gas

\footnotetext{
* Some of the data presented herein were obtained at the W.M. Keck Observatory, which is operated as a scientific partnership among the California Institute of Technology, the University of California, and the National Aeronautics and Space Administration. The Observatory was made possible by the generous financial support of the W.M. Keck Foundation. Based in part on data collected at Subaru Telescope, which is operated by the National Astronomical Observatory of Japan.

5 NSF Graduate Research Fellow.
}

and ice giants in the solar system $(e<0.05)$. The eccentricityperiod distribution of exoplanets within $\approx 3$ au is consistent with having been shaped by tidal circularization at short orbital periods and planet-planet scattering at longer orbital periods, with more massive planets having higher eccentricities on average (e.g., Chatterjee et al. 2008; Winn \& Fabrycky 2015). Ma \& Ge (2014) find that this trend continues into the brown dwarf regime: companions above $\approx 40 M_{\text {Jup }}$ and beyond the tidal circularization radius exhibit an approximately flat eccentricity distribution resembling the orbits of binary stars (e.g., Raghavan et al. 2010; Duchêne \& Kraus 2013). This similarity suggests that high-mass brown dwarfs within a few au predominantly form like stellar binaries.

At wider separations, high-contrast imaging has uncovered over 100 substellar companions spanning a large range of separations $(\approx 5-8000 \mathrm{au})$ and masses $\left(\approx 2-75 M_{\text {Jup }} ;\right.$ see compilations by, e.g., Zuckerman \& Song 2009; Faherty et al. 2010; Bowler 2016; Chauvin 2018, and Deacon et al. 2014). Many formation routes have been proposed for these brown dwarf and planetary-mass companions: core-nucleated or pebble-assisted accretion (e.g., Pollack et al. 1996; Alibert et al. 2005; Lambrechts \& Johansen 2012, 2014), gravitational instabilities in protoplanetary disks (e.g., Boss 1997; Durisen et al. 2007; Vorobyov 2013), fragmentation of collapsing molecular cloud cores (e.g., Boss 2001; Bate et al. 2002; Bate 2009) and gravitational outward scattering 
Table 1

Observations and Astrometry of Substellar Companions

\begin{tabular}{|c|c|c|c|c|c|c|c|c|c|}
\hline Name & $\begin{array}{l}\text { Telescope/ } \\
\text { Instrument }\end{array}$ & $\begin{array}{l}\text { UT Date } \\
\text { (Y-M-D) }\end{array}$ & $\begin{array}{l}\text { Epoch } \\
\text { (UT) }\end{array}$ & $\begin{array}{c}\text { Filter/ } \\
\text { Coronagraph }\end{array}$ & $N \times \underset{(\mathrm{s})}{\text { Coadds }} \times t_{\exp }$ & $\begin{array}{l}\theta_{\text {rot }} \\
\left(^{\circ}\right)\end{array}$ & $\begin{array}{l}\text { Separation } \\
\quad \text { (mas) }\end{array}$ & $\begin{array}{l}\text { P.A. } \\
\left({ }^{\circ}\right)\end{array}$ & $\begin{array}{l}\text { Comp. } \\
\mathrm{S} / \mathrm{N}\end{array}$ \\
\hline HD 49197 B & $\begin{array}{l}\text { Subaru/ } \\
\text { HiCIAO }\end{array}$ & 2011 Dec 28 & 2011.990 & $K_{S} / \operatorname{cor} 300$ & $60 \times 1 \times 30$ & 19 & $913 \pm 15$ & $77.1 \pm 0.7$ & 26 \\
\hline HD 49197 B & Keck/NIRC2 & 2014 Dec 04 & 2014.924 & $K_{S} /$ cor600 & $40 \times 1 \times 60$ & 17 & $875 \pm 5$ & $75.9 \pm 0.3$ & 816 \\
\hline HD 49197 B & Keck/NIRC2 & 2016 Mar 22 & 2016.223 & $K_{S} /$ cor600 & $40 \times 2 \times 15$ & 11 & $874 \pm 5$ & $76.5 \pm 0.3$ & 488 \\
\hline HD 49197 B & Keck/NIRC2 & 2018 Jan 30 & 2018.080 & $K_{S} / \operatorname{cor} 600$ & $27 \times 1 \times 30$ & 15 & $845 \pm 5$ & $76.1 \pm 0.3$ & 84 \\
\hline GJ 504 B & Keck/NIRC2 & 2016 Mar 22 & 2016.223 & $H / \operatorname{cor} 600$ & $100 \times 10 \times 3$ & 91 & $2504 \pm 5$ & $322.7 \pm 0.4$ & 13 \\
\hline GJ 504 B & Keck/NIRC2 & 2018 Jan 30 & 2018.081 & $H / \operatorname{cor} 600$ & $160 \times 10 \times 3$ & 119 & $2503 \pm 5$ & $320.8 \pm 0.3$ & 16 \\
\hline HD 19467 B & Keck/NIRC2 & 2018 Jan 30 & 2018.080 & $H / \operatorname{cor} 600$ & $81 \times 6 \times 5$ & 23 & $1628 \pm 5$ & $239.5 \pm 0.3$ & 26 \\
\hline$\kappa$ And B & Keck/NIRC2 & 2016 Jun 27 & 2016.489 & $H / \operatorname{cor} 600$ & $10 \times 10 \times 2$ & 3 & $965 \pm 5$ & $51.3 \pm 0.3$ & 9 \\
\hline HD $1160 \mathrm{~B}$ & Keck/NIRC2 & 2018 Jan 30 & 2018.080 & $K_{S} / \operatorname{cor} 600$ & $17 \times 1 \times 5$ & $\cdots$ & $790 \pm 5$ & $245.1 \pm 0.3$ & 31 \\
\hline $\begin{array}{r}\text { 1RXS0342 } \\
+1216 \mathrm{~B}\end{array}$ & Keck/NIRC2 & 2018 Jan 30 & 2018.080 & $K_{S} / \operatorname{cor} 600$ & $12 \times 1 \times 20$ & $\cdots$ & $772.3 \pm 1.8$ & $19.6 \pm 0.10$ & 360 \\
\hline CD-35 2722 B & Keck/NIRC2 & 2018 Jan 30 & 2018.080 & $K_{S} / \operatorname{cor} 600$ & $10 \times 1 \times 30$ & $\cdots$ & $2925 \pm 2$ & $241.07 \pm 0.10$ & 116 \\
\hline DH Tau B & Keck/NIRC2 & 2018 Jan 30 & 2018.080 & $K_{S} / \operatorname{cor} 600$ & $3 \times 1 \times 60$ & $\ldots$ & $2354 \pm 2$ & $138.46 \pm 0.10$ & 90 \\
\hline HD 23514 B & Keck/NIRC2 & 2018 Jan 30 & 2018.080 & $K_{S} / \operatorname{cor} 600$ & $16 \times 1 \times 30$ & $\cdots$ & $2648 \pm 2$ & $227.13 \pm 0.10$ & 34 \\
\hline Ross 458 B & Keck/NIRC2 & 2016 Mar 22 & 2016.223 & $K_{S}$ & $10 \times 100 \times 0.014$ & $\cdots$ & $505.4 \pm 1.7$ & $51.63 \pm 0.10$ & 33 \\
\hline Ross 458 B & Keck/NIRC2 & 2018 Jan 30 & 2018.081 & $K_{S}$ & $39 \times 100 \times 0.01$ & $\cdots$ & $361.6 \pm 1.7$ & $21.71 \pm 0.10$ & 10 \\
\hline TWA 5 B & Keck/NIRC2 & 2018 Jan 30 & 2018.081 & $K_{S} / \operatorname{cor} 600$ & $10 \times 1 \times 10$ & $\cdots$ & $1852.1 \pm 1.9$ & $353.09 \pm 0.10$ & 60 \\
\hline $2 \mathrm{M} 1559+4403 \mathrm{~B}$ & Keck/NIRC2 & 2018 Jan 30 & 2018.081 & $K_{S} / \operatorname{cor} 600$ & $10 \times 10 \times 3$ & $\cdots$ & $5609 \pm 3$ & $284.27 \pm 0.10$ & 646 \\
\hline $\begin{array}{l}1 \mathrm{RXS} 2351 \\
+3127 \mathrm{~B}\end{array}$ & Keck/NIRC2 & 2019 Jul 07 & 2019.513 & $H / \operatorname{cor} 600$ & $5 \times 1 \times 30$ & $\cdots$ & $2395 \pm 2$ & $90.71 \pm 0.10$ & 195 \\
\hline
\end{tabular}

by closer-in, higher-mass companions (e.g., Boss 2006; Scharf \& Menou 2009; Veras et al. 2009). Each formation mechanism operates over large overlapping windows of companion mass, orbital separation, and time, which has made it difficult to observationally distinguish the dominant origin of this population. For example, the discovery of extremely low-mass (but high mass ratio) binaries implies that cloud fragmentation can produce few- $M_{\text {Jup }}$ objects at the opacity limit for fragmentation (e.g., 2M1207-3932 b, Chauvin et al. 2004; 2M0441+2301 Bb, Todorov et al. 2010; Bowler \& Hillenbrand 2015; 2M1119-1137 AB, Best et al. 2017) ${ }^{6}$, whereas the orbital architectures of some directly imaged planetary systems indicate that they formed within a disk (e.g., HR 8799 bcde, Marois et al. 2008, 2010; $\beta$ Pic b, Lagrange et al. 2010; HD 95086 b, Rameau et al. 2013a, 2016; Chauvin et al. 2018).

These formation channels predict two broad patterns for the orbital eccentricities of companions: objects that assembled in protoplanetary disks (and without subsequent orbital evolution) should have low eccentricities, whereas those that formed from cloud fragmentation or migrated via outward scattering should exhibit a broad range of eccentricities (Ambartsumian 1937; Veras et al. 2009; Bate 2012). The orbital periods of most widely bound substellar companions are prohibitively long$1000 \mathrm{yr}$ at $100 \mathrm{au}$ for a Sun-like host star and over $10^{4} \mathrm{yr}$ at $500 \mathrm{au}$ - to detect orbital motion given the limited time baselines since their discoveries. Indeed, orbital motion has only been measured for a few substellar companions beyond 100 au (e.g., GQ Lup B, Ginski et al. 2014; Wu et al. 2017; ROXs12 B, Bryan et al. 2016a; GSC 6214-210 B, Pearce et al. 2019). On the other hand, the majority of imaged planets and brown dwarfs within 100 au have shown slight but significant orbital motion after only a few years of monitoring.

\footnotetext{
6 The discovery of isolated planetary-mass objects bolsters this conclusion, although these objects could also be ejected planets (e.g., Forgan et al. 2014).
}

In this study we combine new adaptive optics (AO) imaging observations of substellar companions with astrometry from the literature to uniformly constrain the orbits and underlying population-level eccentricity distributions of directly imaged giant planets $\left(\lesssim 15 M_{\text {Jup }}\right)$ and brown dwarf companions $\left(\approx 15-75 M_{\text {Jup }}\right)$. Each system typically traces out a short orbit arc, resulting in a broad eccentricity posterior distribution, but assembling them into a large sample allows us to infer population-level properties of these objects using hierarchical Bayesian inference.

In Section 2 we describe our AO imaging from Keck Observatory and Subaru Telescope. Our updated orbit fits for systems with new data are summarized in Section 3. We provide uniform orbit fits for our sample of substellar companions and present results using hierarchical Bayesian modeling in Section 4. Implications are discussed in the broader context of formation scenarios in Section 5. We summarize our findings in Section 6.

\section{Observations}

\subsection{Subaru/HiCIAO Adaptive Optics Imaging}

We targeted HD 49197 using the High Contrast Instrument for the Subaru Next Generation Adaptive Optics (HiCIAO; Hodapp et al. 2008; Suzuki et al. 2010) near-infrared imager coupled with the AO188 AO system (Hayano et al. 2010) at Subaru Telescope on UT 2011 December 28 (see Table 1 for details). Conditions were photometric, but the seeing was poor and variable; UKIRT reported $K$-band natural seeing measurements between 1."5 and $2^{\prime \prime}$ during our observations. We acquired a total of 60 frames in $K_{S}$ band, each with an integration time of $30 \mathrm{~s}$. Observations were taken using natural guide star AO in pupil-tracking mode (angular differential imaging; Liu 2004; Marois et al. 2006), which uses field rotation to distinguish speckles from real point sources. HD 49197 was placed behind the 300 mas diameter opaque Lyot 
coronagraph during the ADI sequence, which spanned $19^{\circ}$ of sky rotation.

Data reduction and point-spread function (PSF) subtraction follow the steps detailed in Bowler et al. (2015a). Systematic bias stripes from the detector readout electronics are measured and subtracted, cosmic rays and bad pixels are removed, then images are divided by a normalized flat field. The $K_{S}$-band distortion solution from Bowler et al. (2015a) is applied to each image to correct for optical aberrations. The corresponding $K_{S}$-band plate scale of $9.67 \pm 0.03$ mas pixel $^{-1}$ is adopted for this data set. The typical residual $\mathrm{rms}$ on the distortion correction is 1.2 pix, or 11.6 mas. Celestial North was found to be aligned with the detector columns to within the measurement errors, so no rotation was applied and a value of $0^{\circ} 0 \pm 0.1$ is adopted. Images are registered using a $2 \mathrm{D}$ elliptical Gaussian fit to the PSF wings surrounding the coronagraph and then assembled into a data cube. Finally, PSF subtraction is carried out using "aggressive" and "conservative" implementations of PSF subtraction with the locally optimized combination of images (LOCI; Lafrenière et al. 2007). The aggressive subtraction using LOCI parameters $W=8, N_{A}=300, g=1, N_{\delta}=0.5$, and $d r=2$ produced a higher signal-to-noise ratio $(\mathrm{S} / \mathrm{N})$ for the modest-contrast ( $\Delta K_{S} \approx 8 \mathrm{mag}$ ) companion HD $49197 \mathrm{~B}$, so we adopt this version of the reduction here. The final processed image is shown in Figure 1.

\subsection{Keck/NIRC2 Adaptive Optics Imaging}

We observed 13 targets with substellar companions between 2014 and 2019 (Table 1) with the NIRC2 camera behind natural guide star AO at Keck Observatory (Wizinowich et al. 2000). All observations were acquired with the narrow camera mode, which provides a plate scale of $\approx 10$ mas pix $^{-1}$ and a field of view of 10 ". $2 \times 10$ ". 2 . Observations of HD 19467, $\kappa$ And, GJ 504, and HD 49197 were acquired in pupil-tracking mode to facilitate standard post-processing PSF subtraction. Total on-source integration times ranged from 3 to 81 minutes and the field-of-view rotation angle ranged from $3^{\circ}$ to $119^{\circ}$. The brown dwarf companions to HD 1160, 1RXS0342+1216, CD 35-2722, DH Tau, HD 23514, Ross 458, TWA 5, 2 M1559 +4403 , and 1RXS2351+3127 have lower contrasts and were observed with shorter integration times. The partly transparent 600 mas diameter focal plane coronagraph was used for all systems except Ross 458. Details of the observations can be found in Table 1.

After removing bad pixels and cosmic rays, images are bias subtracted, flat fielded, and corrected for optical distortions using the distortion solution from Yelda et al. (2010) for observations taken before 2015 April and from Service et al. (2016) for observations after that date. The corresponding plate scales are $9.952 \pm 0.002$ mas pixel $^{-1}$ and $9.971 \pm 0.004$ mas pixel $^{-1}$ for these respective dates, and the P.A. offsets are $0.252 \pm 0.009$ and $0.262 \pm 0.020$ with respect to the detector columns. The typical rms residual after distortion correction is $\approx 1$ mas. PSF subtraction for the ADI sequences is carried out following the description in Bowler et al. (2015a): images are first registered using the position of the star visible behind the focal plane mask, then PSF subtraction is performed using LOCI. The mediancombined PSF-subtracted image is then north aligned and a noise map is created by measuring the rms in annuli centered on the host star with a width of 3 pixels. The final PSF-subtracted images and $\mathrm{S} / \mathrm{N}$ maps are shown in Figures 1-5. Note that HD
1160 was observed in field-tracking mode; PSF subtraction for this target entailed derotating to a common pupil angle, implementing PSF subtraction with LOCI, then rerotating to a common sky position angle before coadding the individual frames.

No PSF subtraction is necessary to recover the other substellar companions with modest flux ratios, which are readily visible in the raw frames. After basic image reduction, these frames are registered, north aligned, and then coadded to produce the final images shown in Figure 6.

\subsection{Astrometry and Orbital Motion}

Relative astrometry is measured in each final processed image. Separations in pixels are converted into angular distances using the detector plate scale. Following Bowler et al. (2018), uncertainties take into account random positional measurement errors, estimated to be 0.5 pix for HiCIAO and 0.1 pix for NIRC2 based on end-to-end injection-recovery tests of companions and host star positions in Bowler et al. (2015a) and Bowler et al. (2018); uncertainties in the plate scale; and rms errors from the distortion correction. Similarly, the P.A. uncertainty incorporates random measurement errors, uncertainty in the absolute orientation of celestial north on the detector, rms errors from the distortion correction, and angular uncertainty associated with average azimuthal shearing of the PSF within each image for observations taken in pupil-tracking mode. Bowler et al. (2018) found that systematic errors can dominate the astrometric uncertainty budget for ADI observations with NIRC2 using the 600 mas coronagraph. We therefore adopt conservative errors of 5 mas and 0.3 for our ADI observations in this work. ${ }^{7}$ Our final measurements are reported in Table 1.

The $\mathrm{S} / \mathrm{N}$ of each companion detection is calculated using aperture photometry. Aperture radii of $\approx \lambda / D$ are chosen to encapsulate the central Airy disk and minimize potential effects of oversubtraction at larger radii. Here $D$ is the telescope diameter and $\lambda$ is the central wavelength of the filter. We adopt a 6 pix aperture for observations using HiCIAO in $K_{S}$ band, a 5 pix aperture for NIRC2 in $K_{S}$ band, and a 4 pix aperture for NIRC2 in $H$ band. Noise levels are derived using counts in 100 circular apertures at the same separation but different position angles in each image. These $\mathrm{S} / \mathrm{N}$ measurements range from 9 (for $\kappa$ And B) to 816 for our 2014 epoch of HD 49197 (see Table 1).

Figures 7 and 8 compare our new astrometry (Table 1) to published values in the literature (Appendix A). Orbital motion is clearly detected in many systems and generally extends and refines linear evolution in separation and P.A. with time, with the exception of Ross 458, which has a well-determined orbit. The addition of recent observations is especially important for systems like CD-35 2722 B, for which no astrometry has been published since its discovery by Wahhaj et al. (2011).

There are several instances in which our observations were taken close in time with other published epochs, which provides an opportunity to compare both measurements for mutual consistency. Our astrometry of HD 49197 from UT 2016 March 22 was taken four months after the observations by Bottom et al. (2017) on 2015 November 22 UT with the Stellar Double

\footnotetext{
7 Note that we do not expect systematic errors to be as severe for relative astrometry with short exposures when the companion is bright and the host star is behind the coronagraph mask. See Appendix B of Bowler et al. (2015a) for calibration tests with a binary system.
} 
HD 49197

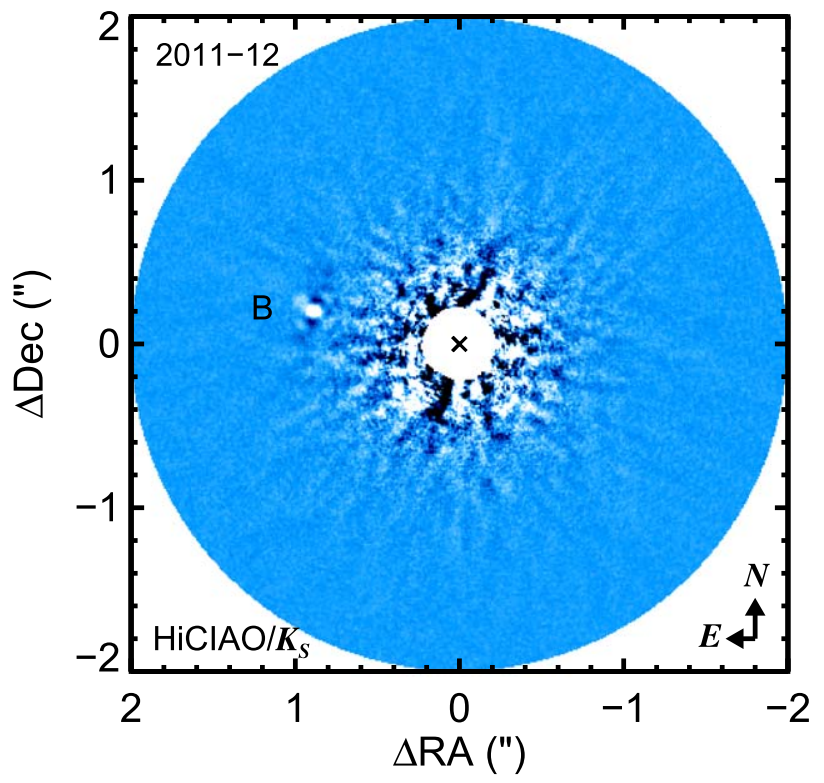

HD 49197

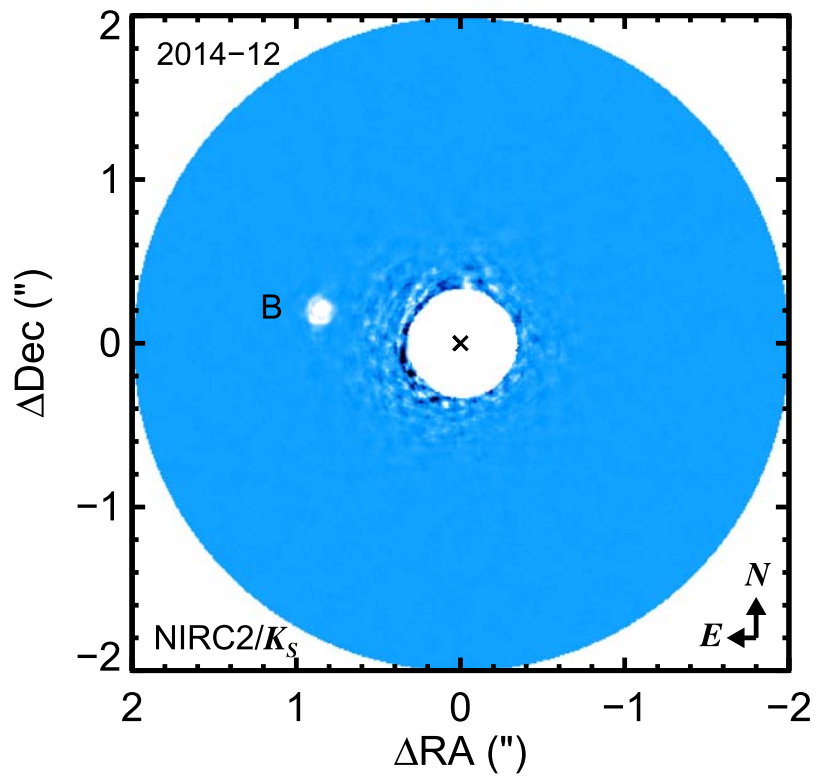

HD 49197

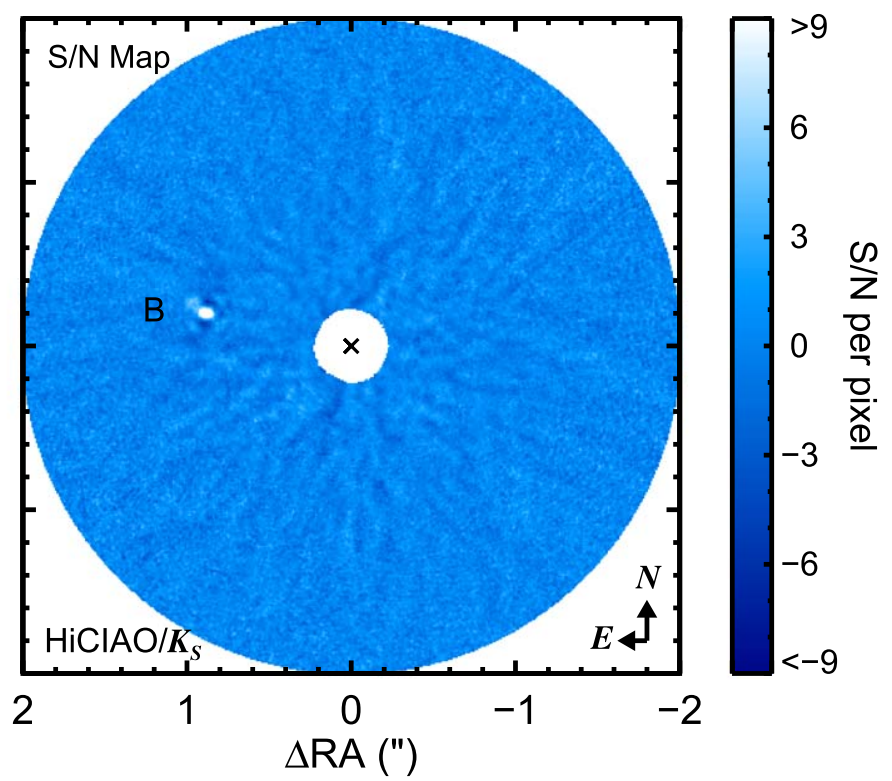

HD 49197

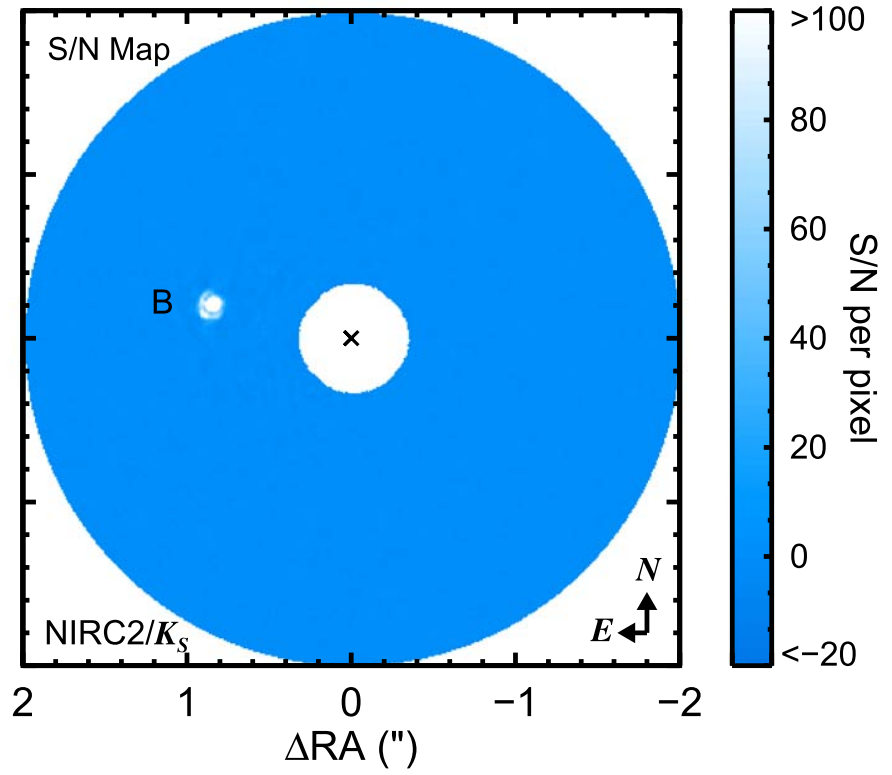

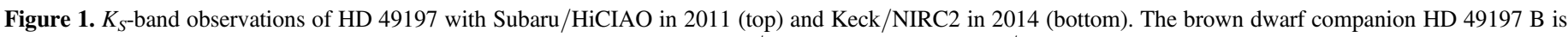

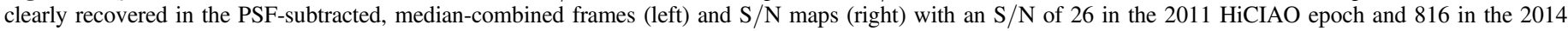
NIRC2 epoch. North is up and east is to the left. The color bars reflect the $\mathrm{S} / \mathrm{N}$ pixel values of the $\mathrm{S} / \mathrm{N}$ maps.

Coronagraph at Palomar Observatory. Our measured separation of $874 \pm 5$ mas and P.A. of $76.5 \pm 0.3$ agrees well with the values of $862 \pm 25$ mas and $76^{\circ} .6 \pm 1.8$ from Bottom et al. We targeted GJ 504 about one week before an observation taken with SPHERE that was reported in Bonnefoy et al. (2018). They find $\left\{\rho=2495 \pm 2\right.$ mas, $\left.\theta=322^{\circ} .48 \pm 0.05\right\}$ on UT 2016 March 29; our measurements of $\{\rho=2504 \pm 5$ mas, $\theta=$ $\left.322^{\circ} .7 \pm 0^{\circ} .4\right\}$ were obtained on UT 2016 March 22. These P.A.s are consistent at the $<1 \sigma$ level, and our separation measurement is consistent within $2 \sigma(9.0 \pm 5.4$ mas $)$. Our UT 2018 January 30 astrometry of HD 1160 B $(\{\rho=790.0 \pm$ 5 mas, $\left.\left.\theta=245^{\circ} .1 \pm 0.3\right\}\right)$ is within $1 \sigma$ of the values also taken with NIRC2 by Currie et al. (2018) on UT 2017 December 9 $\left(\left\{\rho=784 \pm 6\right.\right.$ mas, $\left.\left.\theta=244^{\circ} .9 \pm 0.3\right\}\right)$, about two months before our observations. Despite this good agreement with previous observations, below we attempt to address any potential systematic errors that could result from combining measurements taken with different instruments. This is especially important because systematic errors or underestimated uncertainties can mimic a strong acceleration, which is most readily accounted for in orbit fits through a high eccentricity and time of periastron close to the epoch of observations (see, e.g., results for HR 8799 e from Konopacky et al. 2016).

Linear evolution in astrometry is generally expected for most companions because their orbital periods are long relative to the time baseline of the observations $(\approx 5-20 \mathrm{yr})$. We adopt 
HD 49197

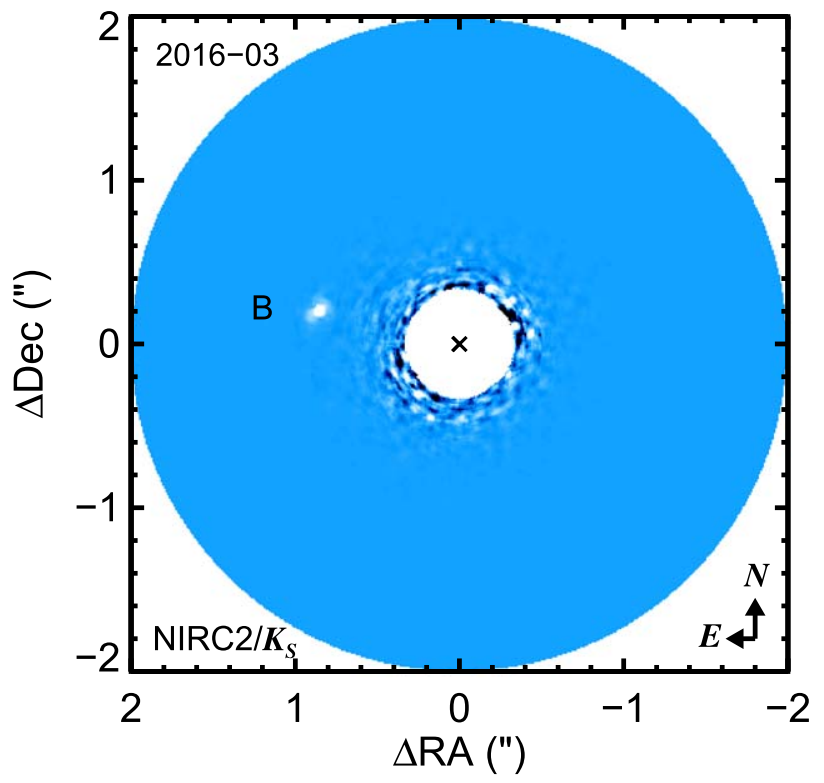

HD 49197

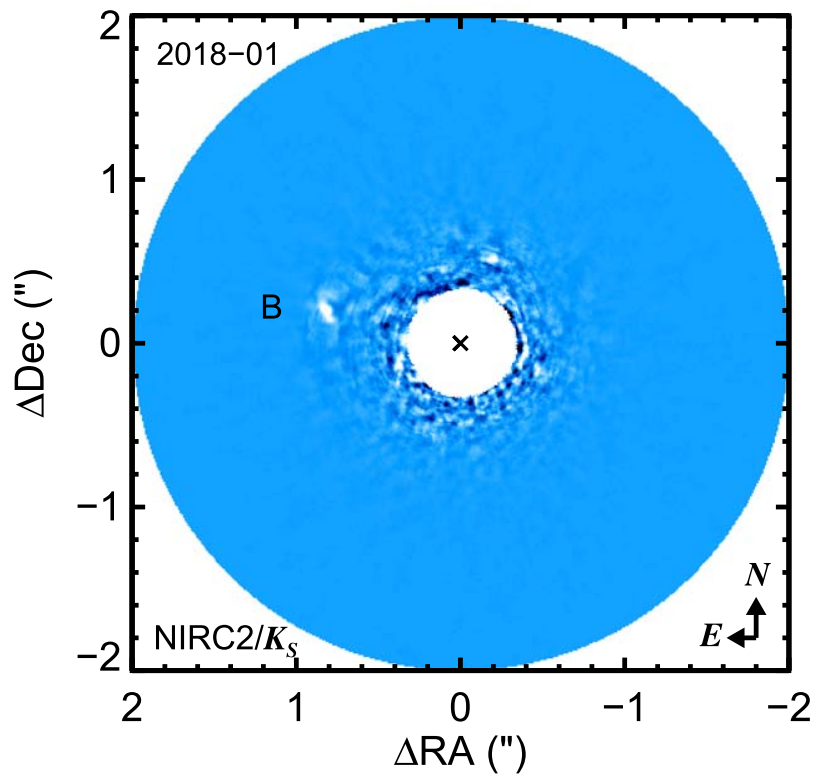

HD 49197

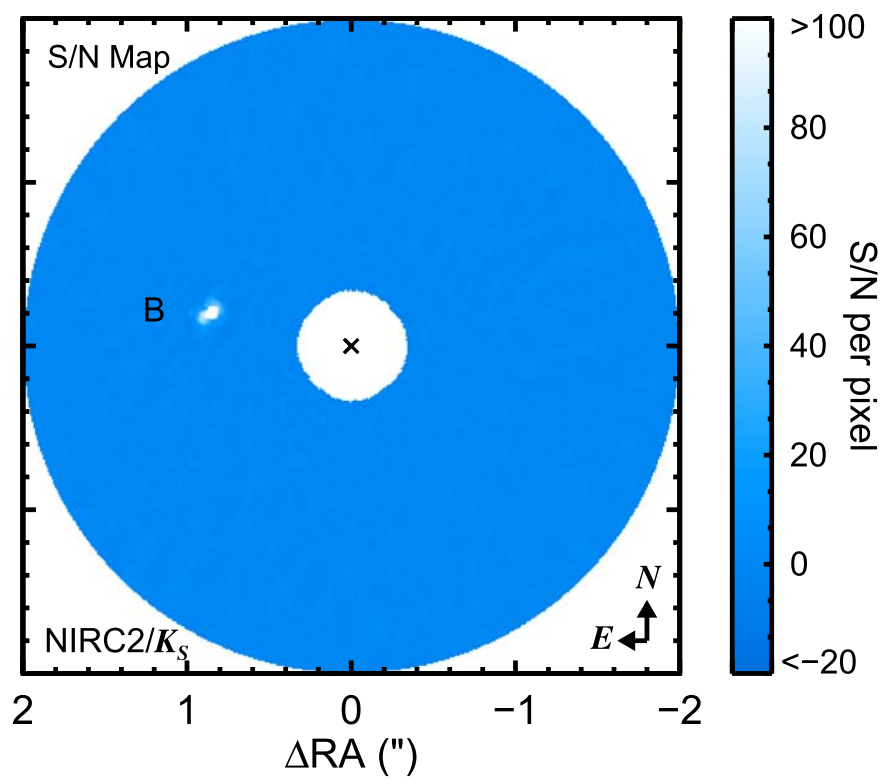

HD 49197

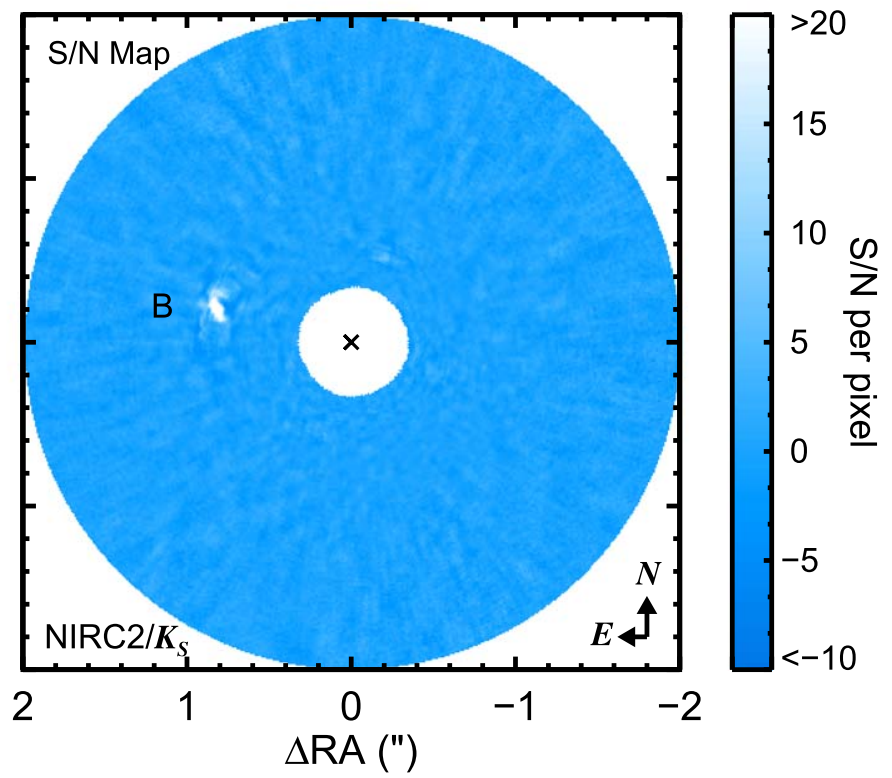

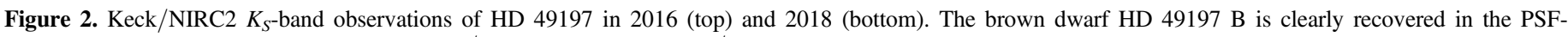

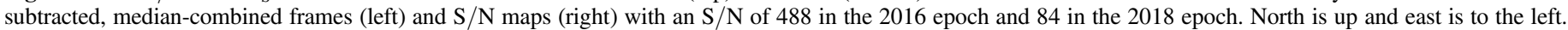
The color bars reflect the $\mathrm{S} / \mathrm{N}$ pixel values of the $\mathrm{S} / \mathrm{N}$ maps.

reduced $\chi^{2}$ values as a metric to assess the fidelity of the astrometric uncertainties, both for our own measurements and for those from the literature. These sample a wide range of instruments, PSF subtraction algorithms, and approaches to measuring astrometry, all of which have the potential to introduce systematic errors in the final astrometry. Linear fits generally provide good matches to the data $\left(\chi_{\nu}^{2} \approx 1\right)$, but in a few instances, the reduced $\chi^{2}$ value is unreasonably high, indicating biased astrometry or underestimated errors. DH Tau is an especially acute example: $\chi_{\nu}^{2}=6.8$ for $\rho(t)$ and $\chi_{\nu}^{2}=96$ for $\theta(t)$. The source of this scatter is most likely instrument-toinstrument calibration errors in distortion correction, plate scale, and north alignment.
To mitigate these biases for our orbit fits, we introduce a "jitter" term added in quadrature with the astrometric uncertainties ( $\sigma_{\mathrm{jit}, \rho}$ for separation and $\sigma_{\mathrm{jit}, \theta}$ for P.A.). This effectively increases the astrometric errors in a systematic fashion until they are consistent with the expected level due to random scatter about linear evolution in separation and P.A. with time (Figures 7 and 8). For fits with $\chi_{\nu}^{2}>1$, jitter is derived by iteratively increasing $\sigma_{\mathrm{jit}, \rho}$ and $\sigma_{\mathrm{jit}, \theta}$ until the linear fits result in $\chi_{\nu}^{2}=1$. Most systems do not require any additional additive error term. When needed, the typical jitter level for separation is $\approx 2-6 \mathrm{mas}$, and for the P.A. it is $\approx 0^{\circ} .1-0.4$. For DH Tau, values of $\sigma_{\mathrm{jit}, \rho}(4.9 \mathrm{mas})$ and $\sigma_{\mathrm{jit}, \theta}$ $(0.74)$ imply that especially strong systematic errors are present 


\section{GJ 504}

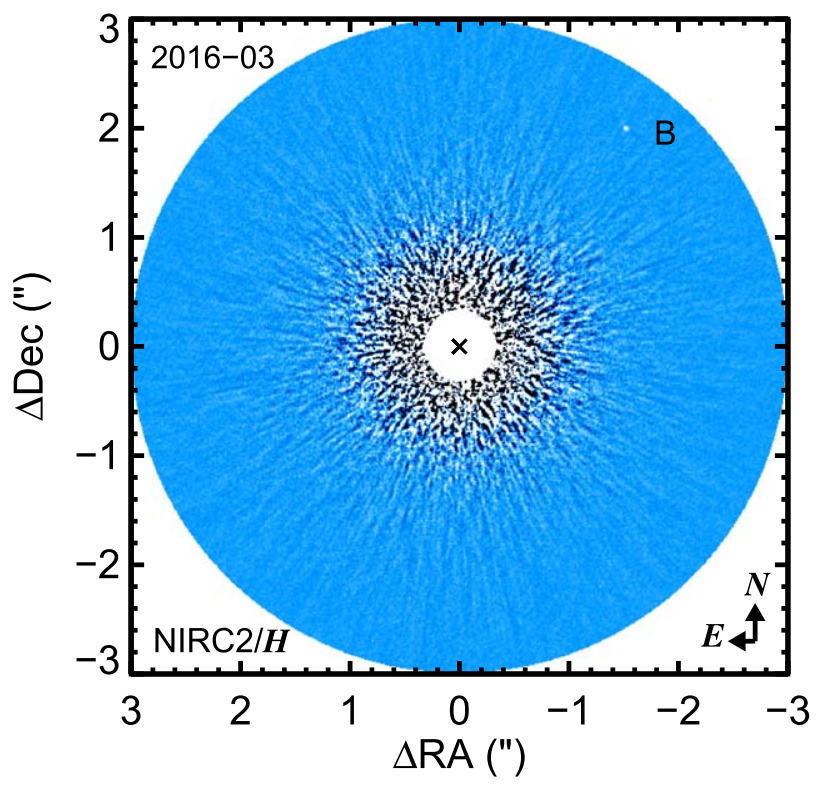

GJ 504

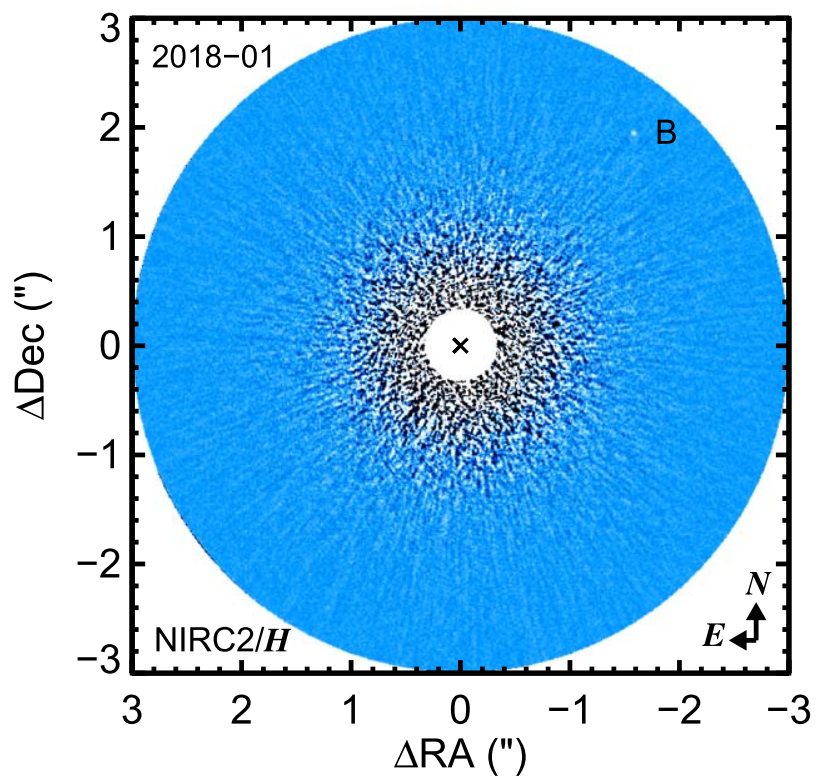

GJ 504

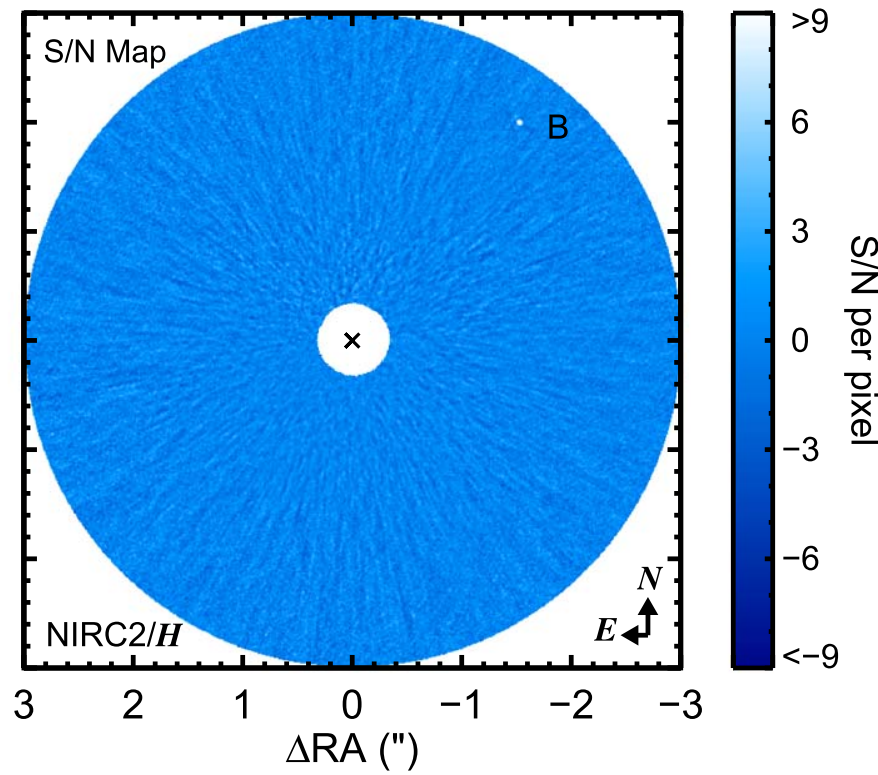

GJ 504

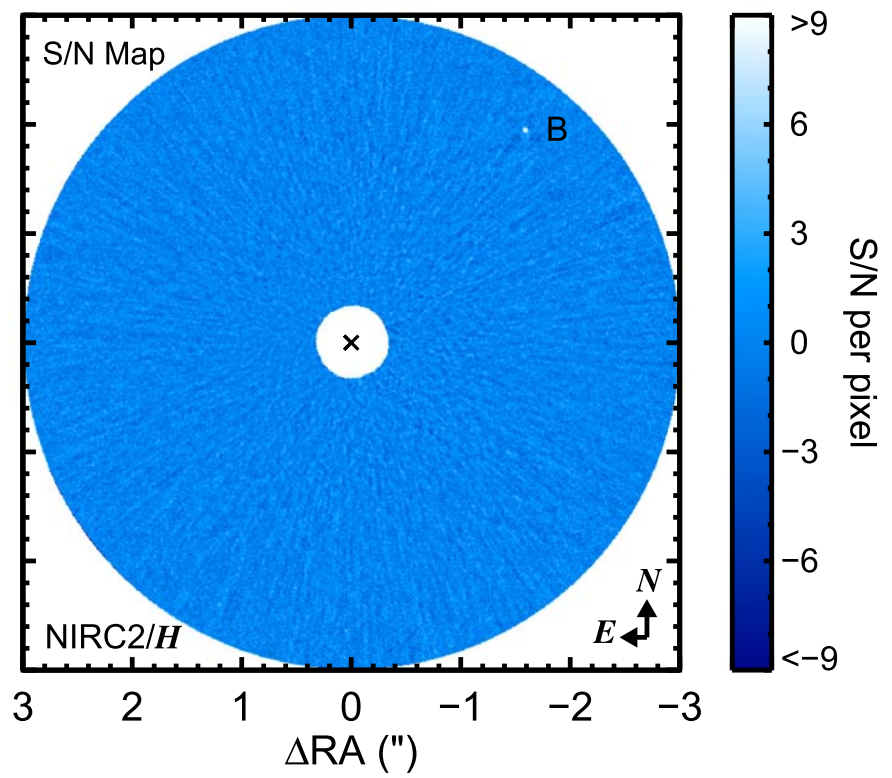

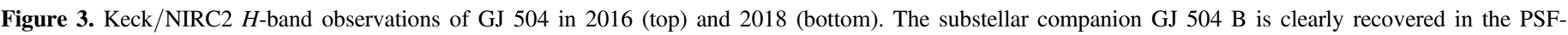

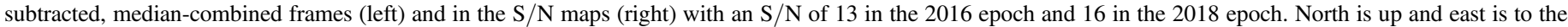
left. The color bars reflect the $S / N$ pixel values of the $S / N$ maps.

in this data set. Linear fits of the separation and P.A. over time incorporating this added uncertainty are provided in Table 2. Residuals of these fits do not show any trends, suggesting the primary source of the high $\chi_{\nu}^{2}$ values is systematic astrometric errors or underestimated uncertainties, rather than our assumption of linear evolution for curved orbital motion (acceleration).

\section{Updated Orbit Fits}

We use our new observations (Table 1) and published astrometry in the literature (Appendix A) to update the Keplerian orbit fits for the 13 systems we observed. In many cases no astrometric epochs have been reported for these systems over the past several years. Our new data reveal slight but significant orbital motion for the majority of targets. In a few instances, these represent the first clear indications of orbital motion for these systems (HD 49197 B, HD 23514 B, $2 \mathrm{M} 1559+4403 \mathrm{~B}$, and 1RXS2351+3127).

Orbit fits are carried out using the orbitize! package for fitting orbits of directly imaged planets ${ }^{8}$ (Blunt et al. 2019). orbitize! implements the Bayesian rejection sampling algorithm Orbits For The Impatient (OFTI), which is detailed in Blunt et al. (2017), and the ptemcee parallel-tempered Markov Chain Monte Carlo (MCMC) approach to sampling posterior distributions from Foreman-Mackey et al. (2013) and

\footnotetext{
$\overline{8 \text { https://github.com/sblunt/orbitize; https://zenodo.org/record/ }}$ 3242703\#.XRfV-5NKiu4
} 
HD 19467

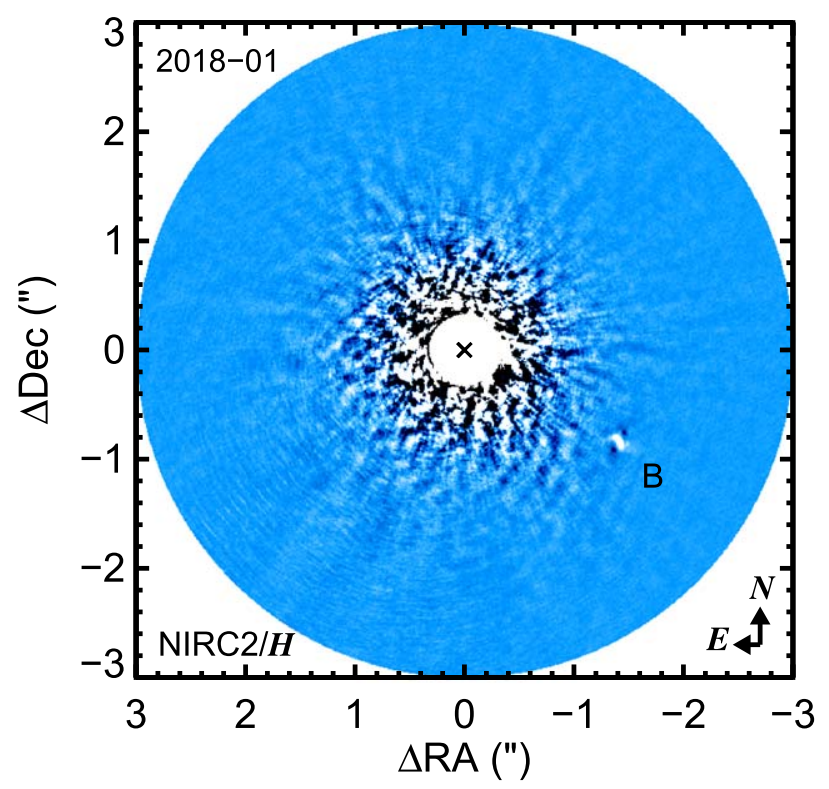

$\kappa$ And

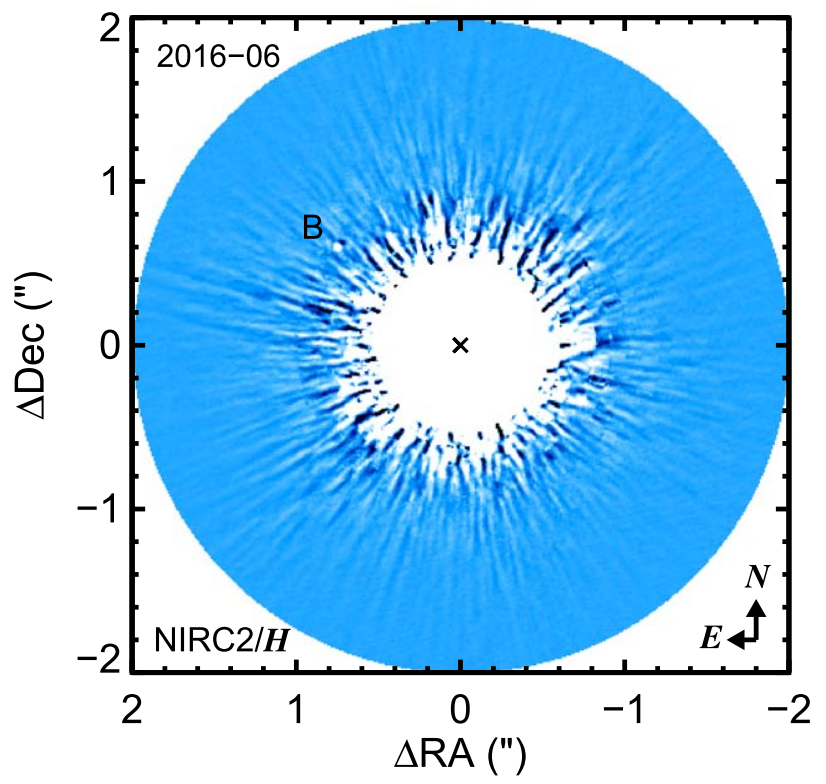

HD 19467

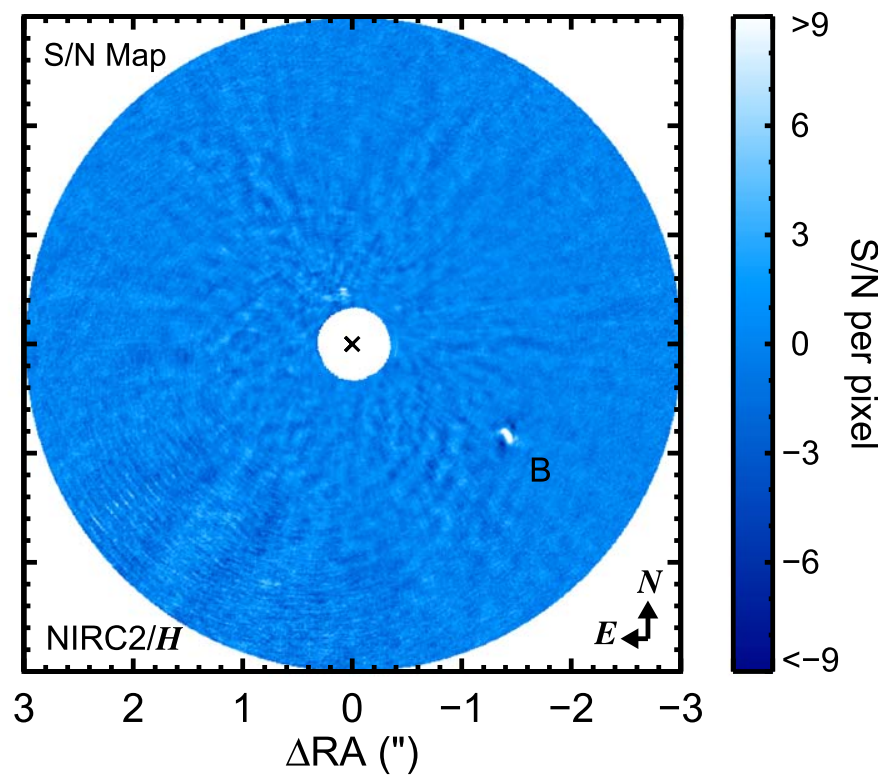

$\kappa$ And

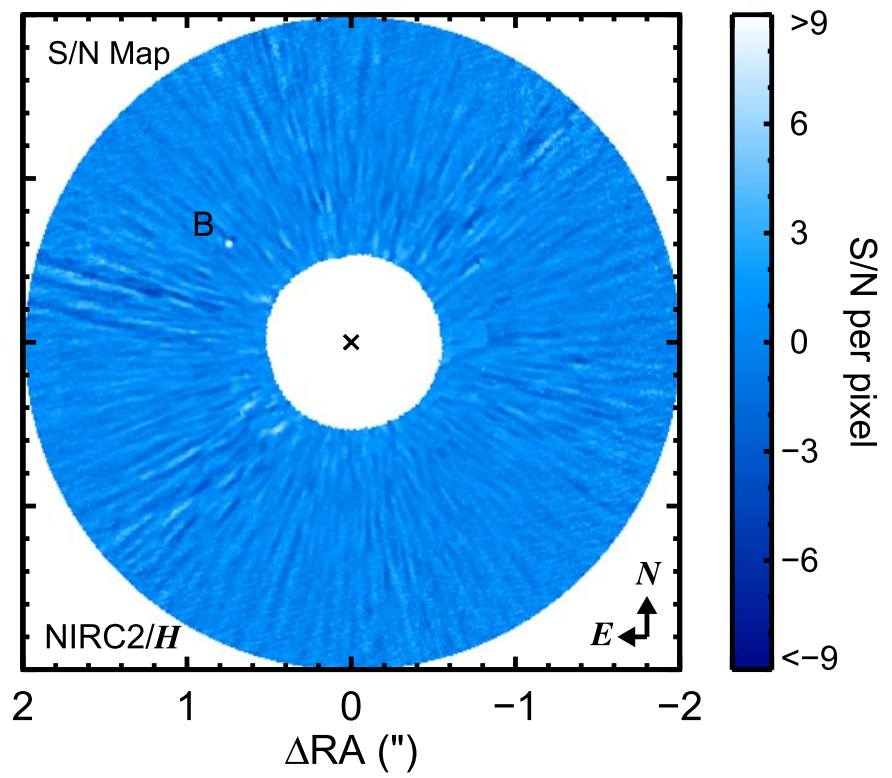

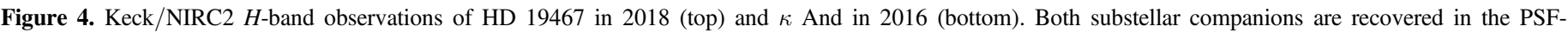

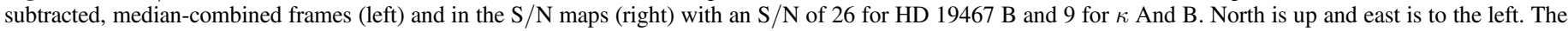
color bars reflect the $\mathrm{S} / \mathrm{N}$ pixel values of the $\mathrm{S} / \mathrm{N}$ maps.

Vousden et al. (2015). OFTI is computationally more efficient than MCMC for mapping complex multimodal posterior shapes, for constraining the orbital elements of systems with relative astrometry that spans only a small fraction of their orbital periods, and when the posteriors are similar to the priors, whereas MCMC is faster for well-constrained orbits. However, both approaches produce similar results when the same parameter priors are used. We use OFTI and MCMC in approximately equal proportions for orbit constraints in this study.

Astrometric errors include jitter values listed in Table 2 for separation and P.A. as described in Section 2.3. The following uninformative priors are adopted for the orbital elements: Jeffreys prior $(1 / a)$ for the semimajor axis (a) from 0.001 to $10^{7} \mathrm{au}$; uniform for the eccentricity $(e)$ from $e=0$ to 1 ; uniform in $\cos (i)$ for the inclination $(i)$ from 0 to $\pi$; uniform for the argument of periastron $(\omega)$; uniform for the longitude of the ascending node $(\Omega)$; and uniform for the time of periastron passage $(\tau)$ from $\tau=0$ to 1 , here expressed in units of period fraction past $\mathrm{MJD}=0$. Stellar masses and parallaxes are allowed to vary but are dominated by the following priors: normal distribution for stellar mass and normal distribution for parallax measurements taken from Gaia DR2 (Gaia Collaboration et al. 2018). ${ }^{9}$

\footnotetext{
9 For orbit fits in this work we assume that the total system mass is equal to the stellar mass plus the mass of the imaged companion.
} 


\section{HD 1160}

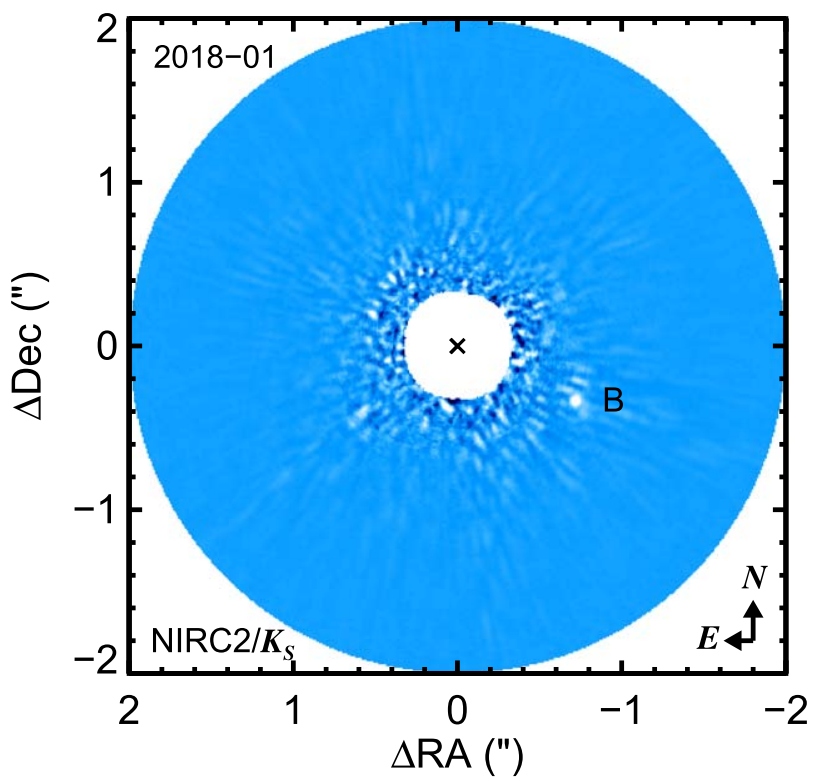

HD 1160

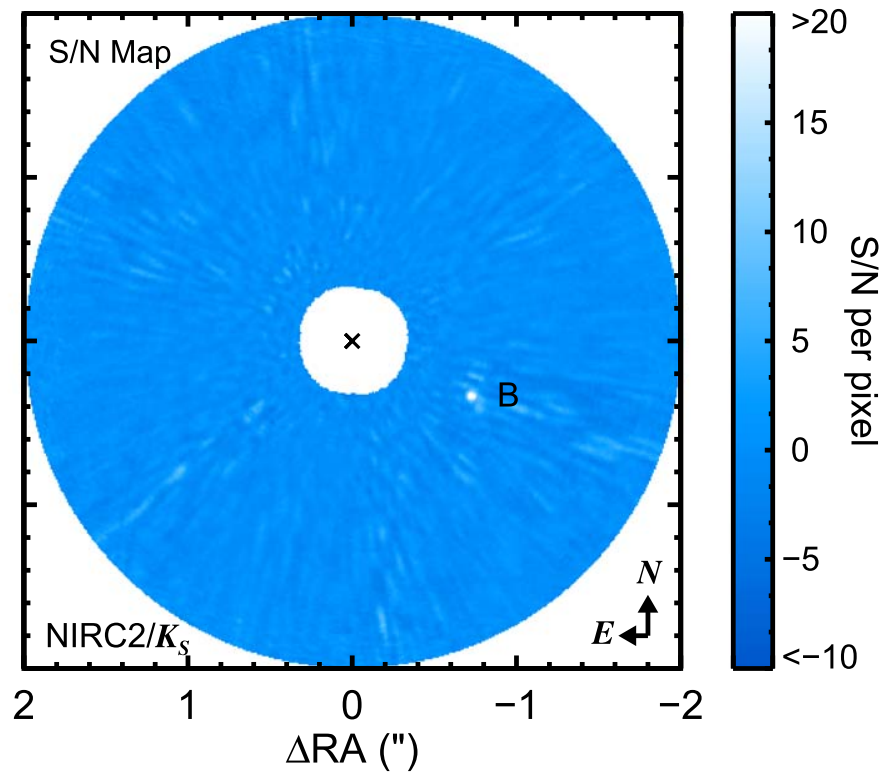

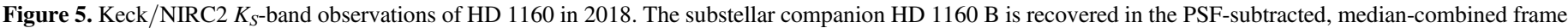
(left) and in the signal-to-noise map (right) with a $\mathrm{S} / \mathrm{N}$ of 31 . North is up and east is to the left. The color bar reflects the $\mathrm{S} / \mathrm{N}$ pixel values of the $\mathrm{S} / \mathrm{N}$ map.

Results for the updated orbit fits using our new astrometry are summarized in Table 3 and described in detail for each system below.

\subsection{HD 49197 B}

Metchev \& Hillenbrand (2004) discovered this companion as part of their Palomar AO search for substellar companions to young stars in the solar neighborhood. They measure a spectral type of L4 \pm 1 from a $K$-band spectrum and infer a mass of $\approx 63 M_{\text {Jup }}$ for HD $49197 \mathrm{~B}$ based on its absolute magnitude. We assume a stellar mass of $1.11 \pm 0.06 M_{\odot}$ from Mints \& Hekker (2017), a total system mass of $1.17 \pm 0.06 M_{\odot}$, and a parallax of $23.99 \pm 0.05$ mas from Gaia DR2 in our orbit analysis.

Two epochs of astrometry were obtained by Metchev \& Hillenbrand (2004) in 2002 and 2003, at which point the separation was $0 . \prime 95$ and P.A. was $\approx 78^{\circ}$. Additional epochs were obtained in 2006 by Serabyn et al. (2009) and in 2015 by Bottom et al. (2017), who first noted orbital motion toward the star. We have been monitoring this companion at a low cadence since 2011 and confirm this motion to smaller separations at a

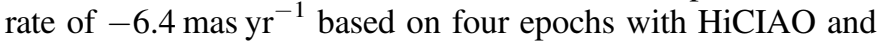
NIRC2. We also detect significant evolution in P.A. for the first time at a rate of $-0^{\circ} .12 \mathrm{yr}^{-1}$ in the clockwise direction. Significant astrometric jitter is required to lower $\chi_{\nu}^{2}$ to $\approx 1$ for the separation measurements $\left(\sigma_{\mathrm{jit}, \rho}=4.6 \mathrm{mas}\right)$. Overall, HD 49197 B has moved inward by about 0 " 1 and moved by about $2^{\circ}$ over the past $15 \mathrm{yr}$.

Our best-fitting orbital solution has a semimajor axis of $29_{-10}^{+7}$ au and an orbital period of $150_{-70}^{+50} \mathrm{yr}(2 \sigma$ credible interval: $80-450 \mathrm{yr}$ ). This implies that $\approx 10 \%$ of its orbit has currently been mapped. The eccentricity of HD 49197 B is poorly constrained. Continued monitoring will be needed to refine the orbital elements for this companion. Results from the orbit fit are shown in Figure 9 and Appendix B.

\section{2. $C D-352722 B$}

Wahhaj et al. (2011) identified this young L4 \pm 1 brown dwarf companion as part of the Gemini NICI Planet-Finding Campaign (Liu et al. 2010). The host is an M1 member of the $\approx 120 \mathrm{Myr} A B$ Dor association, implying a mass of $31 \pm 8 M_{\text {Jup }}$ for CD-35 2722 $\mathrm{B}$ based on its luminosity and young age. We adopt a host mass of $0.40 \pm 0.05 M_{\odot}$ from Wahhaj et al. (2011), a total system mass of $0.43 \pm 0.05 M_{\odot}$, and a parallax of $44.635 \pm 0.026$ mas from Gaia DR2 in our orbit analysis.

Wahhaj et al. presented two epochs from 2009 and 2010, but no additional astrometry has been reported since then. Our epoch from 2018 reveals significant orbital motion at a rate of $-27{\text { mas } \mathrm{yr}^{-1}}^{-1}$ toward the host star in separation and $-0.3 \mathrm{yr}^{-1}$ in P.A. in the clockwise direction (Figure 7). A modest level of additional jitter is needed- $\sigma_{\mathrm{jit}, \rho}=3.5$ mas and $\sigma_{\mathrm{jit}, \theta}=0.40$-although we note that with only three epochs of data, it is difficult to precisely determine the magnitude of any systematic error.

Our orbit fit is shown in Figure 9 and Appendix B and summarized in Table 3 . We find a semimajor axis of $240_{-130}^{+90}$ au, a high eccentricity of $\approx 0.94$, and an orbital period of $5400_{-3900}^{+2900}$ yr $(2 \sigma$ credible interval: $1000-32,000 \mathrm{yr})$. These results are largely consistent with the orbit constraints from Blunt et al. (2017), which only made use of the 2009 and 2010 epochs, but our new epoch now excludes low-eccentricity solutions.

\subsection{GJ 504 B}

GJ 504 B was discovered by Kuzuhara et al. (2013) as part of the Strategic Exploration of Exoplanets and Disks with the Subaru Telescope direct imaging survey (Tamura 2016). Depending on the system age $(21 \pm 2$ Myr or $4.0 \pm 1.8 \mathrm{Gyr})$ and assuming hotstart evolutionary models, this T8-T9.5 companion has a mass that may be as low as $\approx 1 M_{\text {Jup }}$ or as high as $\approx 33 M_{\text {Jup }}$ (Bonnefoy et al. 2018). For this study we assume a stellar host mass of 1.10-1.25 $M_{\odot}$ from Bonnefoy et al. (2018), a companion mass 

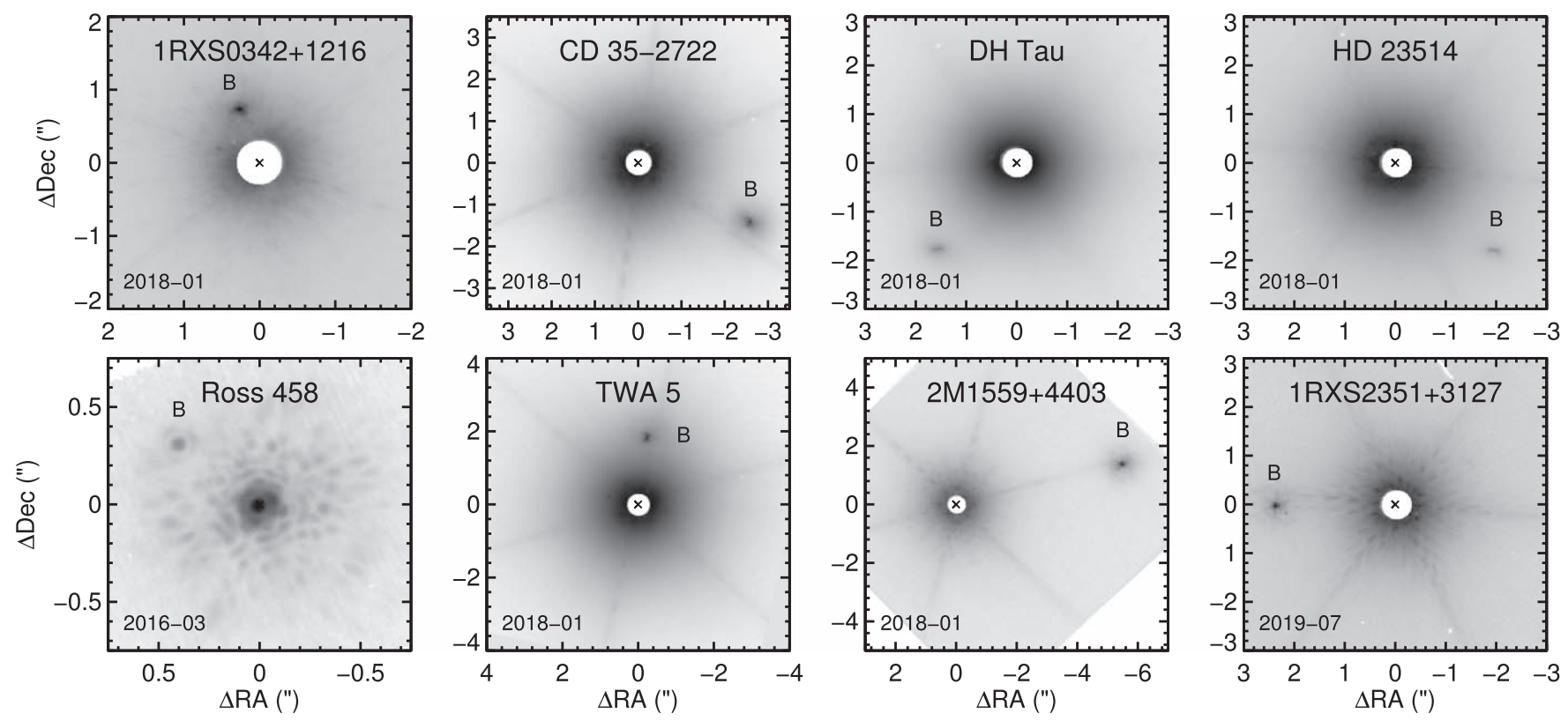

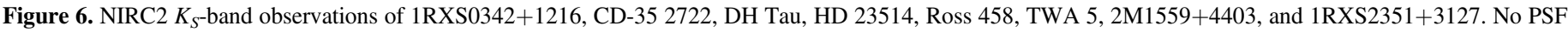
subtraction is needed to recover the substellar companions for these modest-contrast systems. North is up and east is to the left.

of $\approx 23 M_{\text {Jup }}$ from Bonnefoy et al. (2018), a total system mass of $1.20 \pm 0.04 M_{\odot}$, and a parallax of $57.02 \pm 0.25$ mas from Gaia DR2.

Our observations continue the astrometric trends noted by Kuzuhara et al. (2013) and Bonnefoy et al. (2018) from their data spanning 2011 to 2017: GJ 504 B is slowly moving away

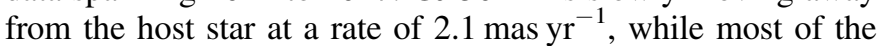
motion is in the P.A. with a rate of $-1^{\circ} .0 \mathrm{yr}^{-1}$ in the clockwise direction. Modest jitter levels of $\sigma_{\mathrm{jit}, \rho}=0.6 \mathrm{mas}$ and $\sigma_{\mathrm{jit}, \theta}=0.03$ are added in quadrature with the available astrometry for this system. Our orbit fit for GJ 504 B indicates a semimajor axis of $41_{-10}^{+4}$ au, a modest eccentricity of $0.22_{-0.17}^{+0.11}$, and a period of $240_{-80}^{+40} \mathrm{yr}$ ( $2 \sigma$ credible interval: $140-470 \mathrm{yr}$ ). Our posteriors generally resemble those from Blunt et al. (2017) and Bonnefoy et al. (2018), with slightly improved constraints due to the extended astrometric coverage. Results from the orbit fit are shown in Figure 9 and Appendix B.

\subsection{HD 19467 B}

HD 19467 B was first imaged by Crepp et al. (2014) based on a long-term radial acceleration of its Sun-like host star. Photometry and spectroscopy from Crepp et al. (2015) imply a mid-T spectral type and a mass of at least $52 M_{\text {Jup. We assume }}$ a stellar mass of $0.95 \pm 0.02 M_{\odot}$ from Crepp et al. (2014), a total system mass of $1.00 \pm 0.02 M_{\odot}$, and a parallax of $31.226 \pm 0.041$ mas from Gaia DR2 in our orbit analysis.

Published astrometry from Crepp et al. (2014) and Crepp et al. (2015) between 2011 and 2014 showed clockwise orbital motion and motion toward the host star. Our new astrometry in 2018 confirms this trend, revealing evolution in P.A. at a rate of $-0.5 \mathrm{yr}^{-1}$ and in separation at a rate of $-5.6 \mathrm{mas} \mathrm{yr}^{-1}$. A modest amount of additional jitter $\left(\sigma_{\mathrm{jit}, \rho}=1.8 \mathrm{mas}, \sigma_{\mathrm{jit}, \theta}=0.06\right)$ is needed to bring the $\chi_{\nu}^{2}$ to unity for a linear evolution of the separation and P.A. HD $19467 \mathrm{~B}$ has an orbital period of $420_{-250}^{+170} \mathrm{yr}$ ( $2 \sigma$ credible interval: $160-1530 \mathrm{yr}$ ) with a semimajor axis of $56_{-25}^{+15}$ au. It does not appear to have a high eccentricity $(e<0.8)$. Results from the orbit fit to the astrometry for this system are shown in Figure 9 and Appendix B.

\section{5. $H D 1160 B$}

Nielsen et al. (2012) identified this low-mass companion to the A0 star HD 1160 as part of the Gemini-NICI PlanetFinding Campaign, along with the low-mass stellar companion HD $1160 \mathrm{C}$ at a wider separation (5"'2). Garcia et al. (2017) found a mid-M spectral type for HD $1160 \mathrm{~B}$ and a mass range between 35 and $90 M_{\text {Jup }}$ depending on the system age. Recently, Curtis et al. (2019) found that this system may belong to the proposed $\approx 120 \mathrm{Myr}$ Psc-Eri stream, which stretches $120^{\circ}$ across the sky and about $400 \mathrm{pc}$ in space. At this age, the implied mass for the companion HD 1160 B would be about $0.12 M_{\odot}$. However, details about the origin, metallicity, membership probabilities, and potential for age gradients in this new proposed stream have not yet been established. For this study we adopt the more uncertain age and mass constraints for HD 1160 B from Nielsen et al. (2012) and Garcia et al. (2017), but caution that this companion may reside above the hydrogen-burning limit if the age is indeed older. The mass we adopt for HD $1160 \mathrm{~A}$ is $1.95 \pm 0.05 M_{\odot}$ (Blunt et al. 2017), the total system mass we use is $2.01 \pm 0.06 M_{\odot}$, and the Gaia DR2 parallax is $7.942 \pm 0.076$ mas.

Astrometry from 2002 to 2018 is presented by Nielsen et al. (2012), Maire et al. (2016), and Currie et al. (2018). Our new epoch in 2018 is consistent with that of Currie et al. (2018), which was taken about two months earlier. We find slow

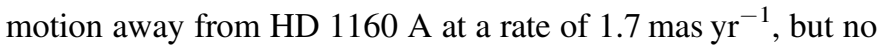
significant change in P.A. over time. There is no evidence that the separation measurement errors are underestimated, but we find that a jitter level of $0^{\circ} .24$ is needed to inflate the $\mathrm{P}$. A. measurements. Our orbit fit (Figure 9 and Appendix B) implies a semimajor axis of $80_{-30}^{+20}$ au and an orbital period of $520_{-270}^{+200} \mathrm{yr}(2 \sigma$ credible interval: $230-3700 \mathrm{yr})$. The eccentricity is poorly constrained. These results are consistent with those from Blunt et al. (2017). 

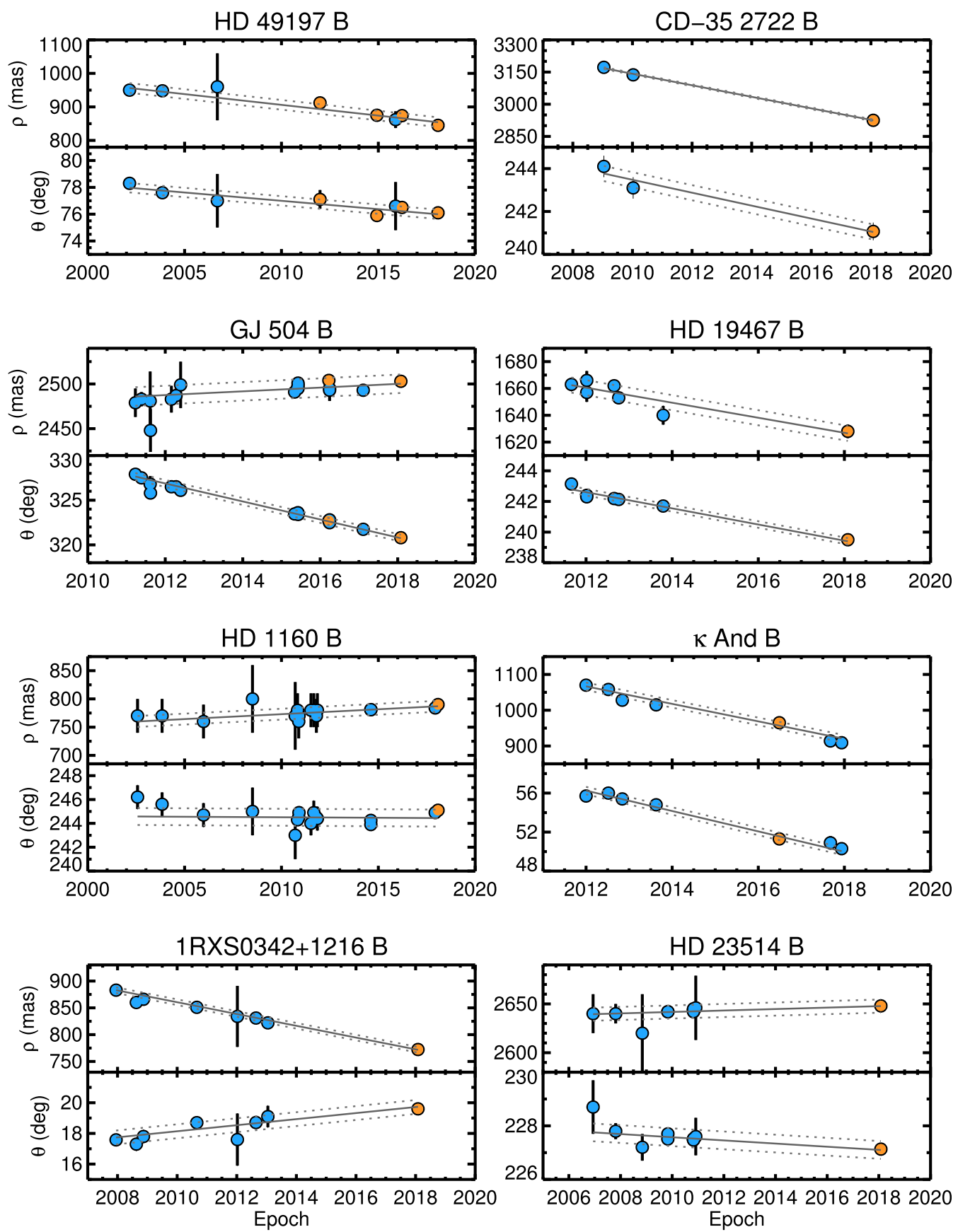

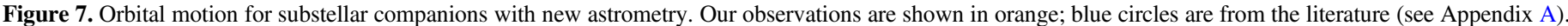

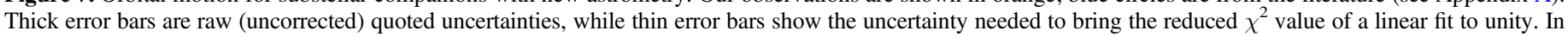

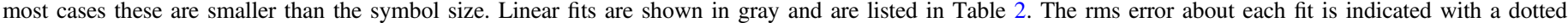
gray line.

\section{6. $\kappa$ And $B$}

$\kappa$ And B (Carson et al. 2013) is a substellar companion orbiting a young B9 star. The companion mass falls near the brown dwarf/planetary-mass boundary at $22 \pm 9 M_{\text {Jup. Fol- }}$ low-up photometry and spectroscopy from Hinkley et al. (2013), Bonnefoy et al. (2014b), and Currie et al. (2018) indicate an early-L dwarf with red colors similar to low-gravity planets and brown dwarfs. We adopt a host star mass of $2.8 \pm 0.1 M_{\odot}$ (Jones et al. 2016), a total system mass of $2.82 \pm 0.10 M_{\odot}$, and a parallax of $19.975 \pm 0.342$ mas from Gaia DR2.
Our astrometry from 2016 combined with measurements from the literature obtained between 2012 and 2018 reveal

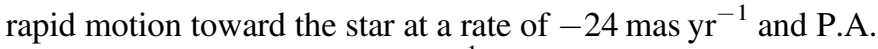
evolution at a rate of $-1.0 \mathrm{yr}^{-1}$ in the clockwise direction. Significant jitter is required for the separation measurements $\left(\sigma_{\mathrm{jit}, \rho}=5.2 \mathrm{mas}\right)$, but no jitter is needed for the P.A. uncertainties. Our orbit fit is shown in Figure 9 and the corner plot is displayed in Appendix B; we find a semimajor axis of $80_{-30}^{+20}$ au, an orbital period of $420_{-210}^{+150} \mathrm{yr}(2 \sigma$ credible interval: $170-1300 \mathrm{yr}$ ), and a high eccentricity of $0.74_{-0.08}^{+0.10}$. These results are consistent with the orbit fit from Currie et al. (2018). 

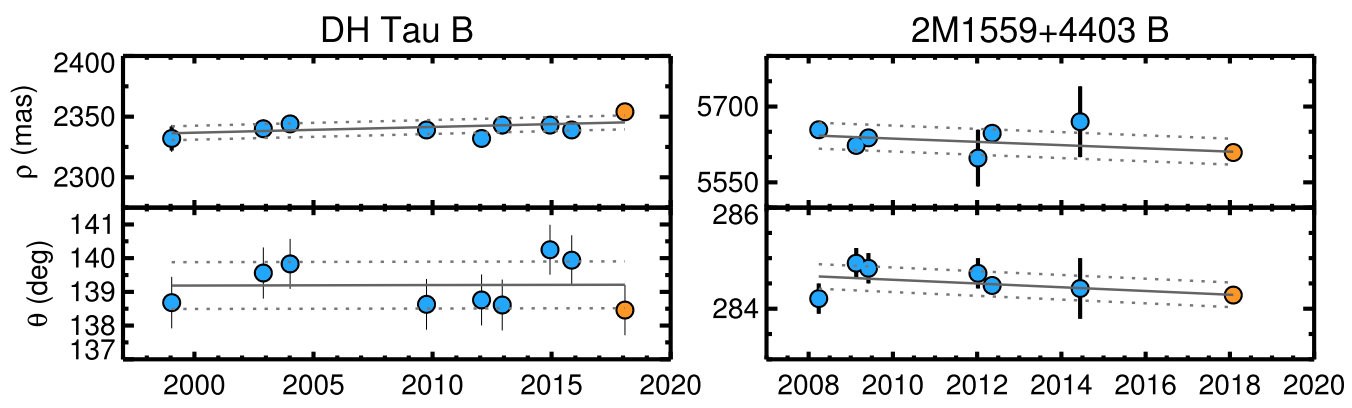

TWA 5 B

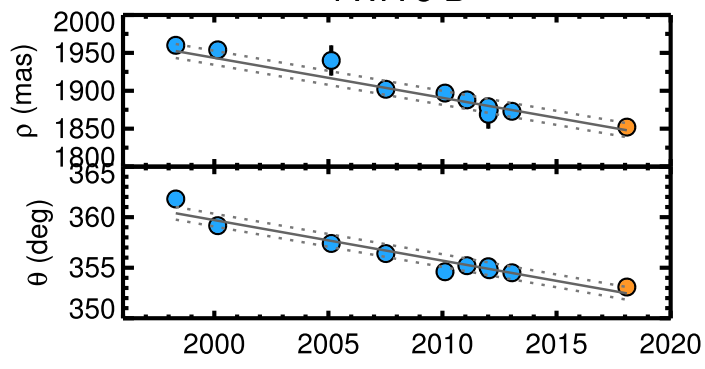

$1 \mathrm{RXS} 2351+3127 \mathrm{~B}$

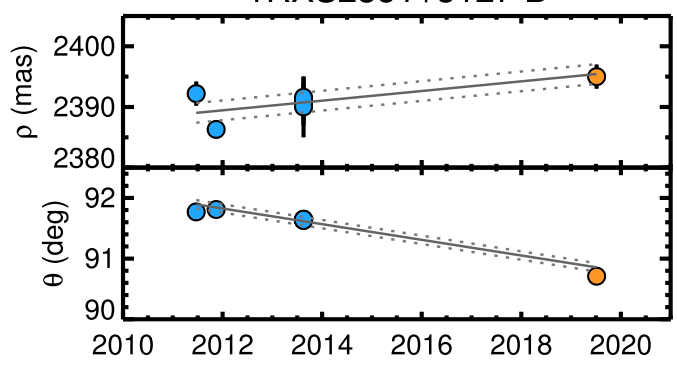

Ross 458 B

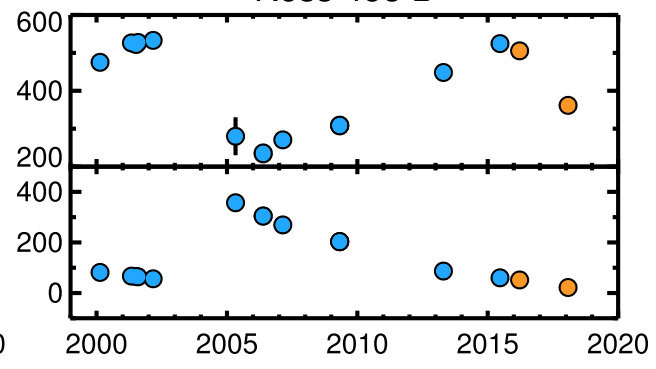

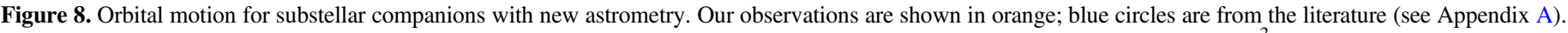

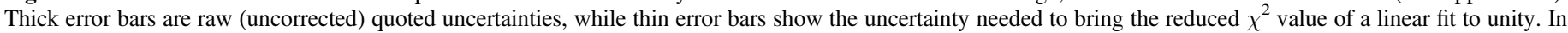

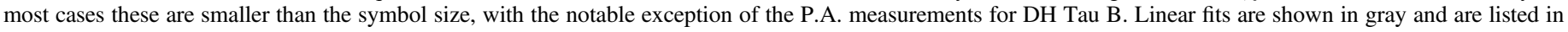
Table 2. The rms error about each fit is indicated with a dotted gray line.

\section{7. $1 R X S 0342+1216 B$}

This $35 \pm 8 M_{\text {Jup }}$ companion to the M4 dwarf $1 \mathrm{RXS}$ J034231.8+121622 (2MASS J03423180+1216225) was discovered by Bowler et al. (2015a) as part of the Planets Around Low-Mass Stars (PALMS) survey. Bowler et al. (2015b) determine a spectral type of L0 from near-infrared spectroscopy and identified significant orbital motion. For our orbit analysis we adopt a host mass of $0.20 \pm 0.05 M_{\odot}$ (Bowler et al. 2015b), a total system mass of $0.23 \pm 0.05 M_{\odot}$, and parallax of $30.308 \pm 0.067$ mas from Gaia DR2.

Our new epoch was obtained in 2018, significantly extending the orbital coverage since the last published epoch acquired in 2013. 1RXS0342+1216 B continues to approach its host star

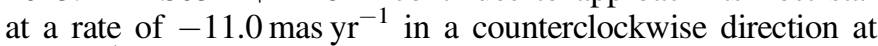
$0.2 \mathrm{yr}^{-1}$. We find that a jitter term of $\sigma_{\mathrm{jit}, \theta}=0.23$ is needed to inflate the P.A. uncertainties. Our orbit fit is shown in Figure 10 and Appendix B; we find a semimajor axis of $37_{-14}^{+8}$ au and an orbital period of $470_{-240}^{+150} \mathrm{yr}(2 \sigma$ credible interval: $160-1040 \mathrm{yr}$ ). At present, the orbital eccentricity is effectively unconstrained.

\subsection{HD $23514 B$}

This substellar companion to the unusually dusty Pleiad HD 23514 was first identified by Rodriguez et al. (2012).
Bowler et al. (2015b) determined a spectral type of M8 for HD 23514 B from $H$ - and $K$-band spectroscopy. We adopt a host star mass of $1.42 \pm 0.15$ from Huber et al. (2016), a total system mass of $1.48 \pm 0.15 M_{\odot}$, and parallax of $7.210 \pm$ 0.054 mas from Gaia DR2.

When combined with published astrometry by Rodriguez et al. (2012) and Yamamoto et al. (2013), our new astrometry from 2018 establishes the first signs of orbital motion for HD $23514 \mathrm{~B}$ : the separation is increasing by $0.7 \mathrm{mas} \mathrm{yr}^{-1}$ and the P.A. is evolving by $-0.06 \mathrm{yr}^{-1}$ in the clockwise direction. Over the $\approx 11$ year baseline since the first detection of this system in 2006, this amounts to a change of about 8 mas and 0 .7. No jitter is necessary for the separation measurements, and only a slight amount $(0.05)$ is needed for the P.A.s. Results from the orbit fit are shown in Figure 10 and Appendix B. Despite the limited orbit coverage, eccentricities above $\approx 0.8$ can be ruled out, and current astrometry favors modest values near 0.3 . The semimajor axis is $420_{-160}^{+100}$ au with a long and highly uncertain orbital period of $7000_{-3900}^{+2500} \mathrm{yr}(2 \sigma$ credible interval: $2100-27,400 \mathrm{yr}$ ).

\subsection{DH Tau B}

Itoh et al. (2005) discovered this brown dwarf companion to DH Tau, a young ( $\approx 2$ Myr) M1 star in Taurus. Bonnefoy et al. (2014a) 
Table 2

Linear Evolution of Separation and P.A.

\begin{tabular}{|c|c|c|c|c|c|c|c|c|c|c|}
\hline $\begin{array}{l}\text { Target } \\
\text { Name }\end{array}$ & $\begin{array}{l}\text { Sep. } a_{0} \\
\text { (mas) }\end{array}$ & $\begin{array}{c}\text { Sep. } a_{1} \\
\left(\operatorname{mas~yr}^{-1}\right)\end{array}$ & $\begin{array}{c}\text { Sep. rms } \\
\text { (mas) }\end{array}$ & $\begin{array}{l}\sigma_{\mathrm{jit}, \rho} \\
\text { (mas) }\end{array}$ & $\begin{array}{c}\text { Sep. } \\
\chi_{\nu}^{2}\end{array}$ & $\begin{array}{l}\text { P.A. } b_{0} \\
\left(^{\circ}\right)\end{array}$ & $\begin{array}{l}\text { P.A. } b_{1} \\
\left({ }^{\circ} \mathrm{yr}^{-1}\right)\end{array}$ & $\begin{array}{l}\text { P.A. rms } \\
\left({ }^{\circ}\right)\end{array}$ & $\begin{array}{c}\sigma_{\mathrm{jit}, \theta} \\
\left(^{\circ}\right)\end{array}$ & $\begin{array}{l}\text { P.A. } \\
\chi_{\nu}^{2}\end{array}$ \\
\hline HD $984 \mathrm{~B}$ & $-24198.3242 \pm 6652.9297$ & $12.1124 \pm 3.3012$ & 13.1 & 3.2 & 0.99 & $17555.8809 \pm 867.8156$ & $-8.6683 \pm 0.4306$ & 0.85 & 0.00 & 0.49 \\
\hline HD $1160 \mathrm{~B}$ & $-2723.1067 \pm 1785.6223$ & $1.7393 \pm 0.8863$ & 9.7 & 0.0 & 0.10 & $261.4736 \pm 72.9082$ & $-0.0084 \pm 0.0362$ & 0.71 & 0.24 & 0.99 \\
\hline HD 19467 B & $12941.7510 \pm 2004.5902$ & $-5.6069 \pm 0.9957$ & 5.6 & 1.8 & 0.99 & $1304.3900 \pm 107.5698$ & $-0.5277 \pm 0.0534$ & 0.21 & 0.06 & 0.99 \\
\hline 1RXS0342+1216 B & $22939.0391 \pm 327.6723$ & $-10.9844 \pm 0.1632$ & 5.3 & 0.0 & 0.94 & $-378.7789 \pm 63.8557$ & $0.1975 \pm 0.0317$ & 0.45 & 0.23 & 0.96 \\
\hline 51 Eri b & $6720.5132 \pm 2516.3901$ & $-3.1086 \pm 1.2477$ & 3.8 & 0.0 & 0.31 & $11322.8174 \pm 343.0927$ & $-5.5345 \pm 0.1701$ & 0.39 & 0.00 & 0.26 \\
\hline CD-35 2722 B & $57221.8008 \pm 1387.3359$ & $-26.9053 \pm 0.6888$ & 4.3 & 3.5 & 0.98 & $849.6935 \pm 127.4335$ & $-0.3016 \pm 0.0633$ & 0.35 & 0.40 & 1.00 \\
\hline HD 49197 B & $13782.7002 \pm 843.4277$ & $-6.4062 \pm 0.4196$ & 14.4 & 4.6 & 0.98 & $327.0609 \pm 44.3513$ & $-0.1244 \pm 0.0220$ & 0.35 & 0.00 & 0.81 \\
\hline HR 2562 B & $-50161.6797 \pm 2145.8904$ & $25.1873 \pm 1.0638$ & 1.2 & 0.0 & 0.28 & $-202.7896 \pm 257.1518$ & $0.2482 \pm 0.1275$ & 0.17 & 0.00 & 0.44 \\
\hline HR 3549 B & $17045.1328 \pm 9505.5195$ & $-8.0338 \pm 4.7161$ & 0.9 & 0.0 & 0.02 & $1324.7207 \pm 498.3914$ & $-0.5798 \pm 0.2473$ & 0.29 & 0.00 & 0.20 \\
\hline HD 95086 b & $-1867.0281 \pm 1941.0756$ & $1.2346 \pm 0.9629$ & 5.2 & 0.0 & 0.31 & $2351.5354 \pm 193.0089$ & $-1.0930 \pm 0.0957$ & 0.32 & 0.00 & 0.32 \\
\hline GJ 504 B & $-1710.9081 \pm 1831.3406$ & $2.0867 \pm 0.9085$ & 10.4 & 0.6 & 0.99 & $2354.0681 \pm 51.8511$ & $-1.0076 \pm 0.0257$ & 0.39 & 0.03 & 1.00 \\
\hline HIP 65426 b & $6849.5601 \pm 2470.6326$ & $-2.9850 \pm 1.2246$ & 8.0 & 0.0 & 0.82 & $511.8435 \pm 213.0702$ & $-0.1794 \pm 0.1056$ & 0.55 & 0.12 & 0.98 \\
\hline PDS $70 \mathrm{~b}$ & $2706.3250 \pm 3142.1650$ & $-1.2465 \pm 1.5588$ & 6.2 & 0.0 & 0.45 & $5107.3291 \pm 817.6153$ & $-2.4576 \pm 0.4055$ & 1.35 & 0.00 & 0.28 \\
\hline PZ Tel B ${ }^{a}$ & $-58956.1328 \pm 835.7415$ & $29.5029 \pm 0.4153$ & 6.0 & 3.8 & 0.98 & $326.9745 \pm 62.2090$ & $-0.1328 \pm 0.0309$ & 0.47 & 0.16 & 0.98 \\
\hline HD 206893 B & $16907.2207 \pm 2168.6890$ & $-8.2524 \pm 1.0749$ & 2.4 & 0.0 & 0.81 & $18744.5137 \pm 537.0878$ & $-9.2639 \pm 0.2663$ & 0.64 & 0.29 & 0.98 \\
\hline$\kappa$ And $\mathrm{B}$ & $50347.6562 \pm 3829.7366$ & $-24.4935 \pm 1.9010$ & 10.6 & 5.2 & 0.99 & $2160.1741 \pm 167.6283$ & $-1.0457 \pm 0.0832$ & 0.38 & 0.00 & 0.63 \\
\hline HD 23514 B & $1142.6167 \pm 531.7578$ & $0.7459 \pm 0.2644$ & 6.7 & 0.0 & 0.39 & $346.3043 \pm 28.8196$ & $-0.0591 \pm 0.0143$ & 0.33 & 0.05 & 0.88 \\
\hline DH Tau B & $1396.2018 \pm 757.6589$ & $0.4703 \pm 0.3766$ & 5.7 & 4.9 & 0.98 & $136.6982 \pm 81.7527$ & $0.0012 \pm 0.0407$ & 0.69 & 0.74 & 0.99 \\
\hline 2M1559+4403 B & $12130.2617 \pm 2763.0696$ & $-3.2307 \pm 1.3737$ & 25.6 & 10.5 & 1.00 & $359.0865 \pm 37.8433$ & $-0.0371 \pm 0.0188$ & 0.24 & 0.00 & 0.82 \\
\hline TWA 5 B & $12507.5928 \pm 623.8889$ & $-5.2820 \pm 0.3101$ & 9.2 & 2.3 & 0.99 & $1161.5425 \pm 68.8053$ & $-0.4009 \pm 0.0342$ & 0.63 & 0.58 & 1.00 \\
\hline 1RXS2351+3127 B & $788.8538 \pm 609.4363$ & $0.7955 \pm 0.3026$ & 1.6 & 0.3 & 0.99 & $352.0048 \pm 30.3868$ & $-0.1293 \pm 0.0151$ & 0.07 & 0.05 & 0.90 \\
\hline
\end{tabular}

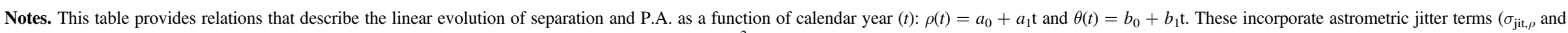
$\left.\sigma_{\mathrm{jit}, \theta}\right)$, which are added in quadrature with the quoted astrometric errors to ensure that the reduced $\chi^{2}$ values are $\leqslant 1.0$.

${ }^{a}$ Note that PZ Tel B shows signs of slight curvature, which is not reflected in this linear fit. 
Table 3

Results from Orbit Fits

\begin{tabular}{|c|c|c|c|c|c|c|c|}
\hline Name & $\begin{array}{c}P \\
(\mathrm{yr})\end{array}$ & $\begin{array}{c}a \\
(\mathrm{au})\end{array}$ & $e$ & $\begin{array}{c}i \\
\left.{ }^{\circ}\right)\end{array}$ & $\begin{array}{l}\omega^{\mathrm{a}} \\
\left({ }^{\circ}\right)\end{array}$ & $\begin{array}{l}\Omega^{\mathrm{a}} \\
\left({ }^{\circ}\right)\end{array}$ & $\overline{\tau^{\mathrm{b}}}$ \\
\hline HD $984 \mathrm{~B}$ & $67.5_{-43}^{+23}(14.6-260)$ & $17.6_{-8.1}^{+4.3}(6.98-43.9)$ & $0.23_{-0.23}^{+0.11}(0.0-0.63)$ & $120_{-7.3}^{+6.1}(108-137)$ & $111_{-41}^{+69}$ & $26.5_{-16}^{+11}$ & $0.504_{-0.36}^{+0.32}$ \\
\hline HD $1160 \mathrm{~B}$ & $523_{-270}^{+200}(228-3700)$ & $81.9_{-31}^{+20}(48.7-302)$ & $0.78_{-0.23}^{+0.22}(0.076-1.0)$ & $92.0_{-9.3}^{+8.7}(61.6-137)$ & $48.1_{-48}^{+20}$ & $64.6_{-3.6}^{+4.8}$ & $0.114_{-0.11}^{+0.070}$ \\
\hline $\begin{array}{r}1 \mathrm{RXS} 0342 \\
+1216 \mathrm{~B}\end{array}$ & $470_{-240}^{+150}(157-1040)$ & $36.5_{-14}^{+8.1}(18.8-61.4)$ & $0.34_{-0.34}^{+0.25}(0.026-0.98)$ & $80.8_{-4.0}^{+4.7}(38.8-87.0)$ & $116_{-42}^{+64}$ & $12.9_{-5.0}^{+3.0}$ & $0.469_{-0.26}^{+0.22}$ \\
\hline 51 Eri b & $34.5_{-15}^{+9.6}(17.1-117)$ & $12.8_{-3.8}^{+2.4}(8.14-28.9)$ & $0.50_{-0.083}^{+0.11}(0.15-0.68)$ & $132_{-10}^{+9.1}(115-156)$ & $85.7_{-27}^{+25}$ & $72.6_{-73}^{+34}$ & $0.503_{-0.24}^{+0.44}$ \\
\hline HD 49197 B & $146_{-67}^{+53}(75.0-449)$ & $29.1_{-9.7}^{+6.7}(19.1-61.8)$ & $0.73_{-0.22}^{+0.27}(0.072-1.0)$ & $96.9_{-4.1}^{+4.1}(91.9-133)$ & $138_{-18}^{+42}$ & $75.6_{-2.5}^{+2.7}$ & $0.503_{-0.50}^{+0.039}$ \\
\hline HR 2562 B & $114_{-78}^{+52}(35.6-630)$ & $\begin{array}{c}26.3_{-14}^{+7.5} \\
(12.2-82.3)\end{array}$ & $0.45_{-0.45}^{+0.28}(0.037-1.0)$ & $86.3_{-2.3}^{+3.2}(59.3-91.7)$ & $125_{-63}^{+55}$ & $301_{-3.3}^{+3.7}$ & $0.439_{-0.44}^{+0.20}$ \\
\hline HR 3549 B & $599_{-380}^{+260}(168-3030)$ & $94.3_{-43}^{+28}(42.1-278)$ & $0.43_{-0.41}^{+0.17}(0.0-0.88)$ & $132_{-21}^{+21}(97.5-172)$ & $87.3_{-87}^{+34}$ & $264_{-46}^{+96}$ & $0.436_{-0.43}^{+0.20}$ \\
\hline HD 95086 b & $350_{-120}^{+90}(175-1030)$ & $59.2_{-13}^{+10}(38.6-122)$ & $0.14_{-0.14}^{+0.067}(0.0-0.48)$ & $150_{-13}^{+12}(127-174)$ & $105_{-38}^{+74}$ & $88.1_{-39}^{+50}$ & $0.271_{-0.21}^{+0.11}$ \\
\hline GJ 504 B & $242_{-84}^{+37}(138-468)$ & $\begin{array}{c}41.3_{-10}^{+4.0} \\
(29.0-64.1)\end{array}$ & $0.22_{-0.17}^{+0.11}(0.0-0.44)$ & $141_{-8.7}^{+8.0}(128-169)$ & $61.0_{-61}^{+37}$ & $153_{-12}^{+9.5}$ & $0.357_{-0.078}^{+0.097}$ \\
\hline PZ Tel B & $108_{-35}^{+39}(61.3-210)$ & $\begin{array}{l}24.8_{-5.5}^{+5.3} \\
(17.6-38.6)\end{array}$ & $0.89_{-0.048}^{+0.10}(0.74-1.0)$ & $93.4_{-1.7}^{+1.2}(91.0-100)$ & $168_{-4.7}^{+12}$ & $57.1_{-0.88}^{+1.2}$ & $0.595_{-0.18}^{+0.40}$ \\
\hline HD 206893 B & $28.1_{-9.2}^{+4.4}(17.4-55.6)$ & $10.2_{-2.3}^{+1.1}(7.43-16.1)$ & $0.25_{-0.14}^{+0.17}(0.0-0.44)$ & $143_{-10}^{+9.7}(128-170)$ & $74.3_{-74}^{+33}$ & $255_{-37}^{+31}$ & $0.522_{-0.20}^{+0.47}$ \\
\hline$\kappa$ And B & $417_{-210}^{+150}(174-1300)$ & $78.9_{-28}^{+18}(44.8-169)$ & $0.74_{-0.075}^{+0.098}(0.53-0.88)$ & $139_{-18}^{+13}(115-170)$ & $134_{-24}^{+35}$ & $72.0_{-16}^{+21}$ & $0.449_{-0.22}^{+0.20}$ \\
\hline HD 23514 B & $\begin{array}{r}7010_{-3900}^{+2500} \\
(2120-27400)\end{array}$ & $\begin{array}{r}417_{-160}^{+100} \\
(201-1040)\end{array}$ & $0.29_{-0.28}^{+0.11}(0.0-0.68)$ & $140_{-16}^{+17}(111-172)$ & $106_{-37}^{+74}$ & $259_{-43}^{+100}$ & $0.740_{-0.10}^{+0.26}$ \\
\hline DH Tau B & $\begin{array}{r}7460_{-4800}^{+3200} \\
(2360-59800)\end{array}$ & $330_{-160}^{+89}(158-1320)$ & $0.49_{-0.49}^{+0.19}(0.0023-0.96)$ & $79.9_{-40}^{+38}(15.5-154)$ & $99.0_{-46}^{+65}$ & $129_{-38}^{+51}$ & $0.623_{-0.18}^{+0.31}$ \\
\hline $2 \mathrm{M} 1559+4403 \mathrm{~B}$ & $\begin{array}{r}6510_{-4300}^{+2800} \\
(1830-33500)\end{array}$ & $289_{-140}^{+80}(131-859)$ & $0.47_{-0.34}^{+0.28}(0.0-0.90)$ & $122_{-21}^{+17}(91.4-165)$ & $79.2_{-79}^{+40}$ & $122_{-21}^{+33}$ & $0.297_{-0.25}^{+0.13}$ \\
\hline TWA $5 \mathrm{~B}$ & $1810_{-840}^{+540}(737-4420)$ & $146_{-45}^{+30}(85.5-265)$ & $0.43_{-0.10}^{+0.099}(0.23-0.67)$ & $151_{-13}^{+12}(129-175)$ & $123_{-31}^{+41}$ & $48.0_{-23}^{+24}$ & $0.159_{-0.095}^{+0.066}$ \\
\hline Ross $458 \mathrm{~B}$ & $\begin{array}{c}13.528_{-0.014}^{+0.02} \\
(13.49-13.56)\end{array}$ & $\begin{aligned} 4.95_{-0.0095}^{+0.0096} \\
(4.93-4.97)\end{aligned}$ & $\begin{array}{l}0.2419_{-0.001}^{+0.001} \\
(0.240-0.244)\end{array}$ & $\begin{array}{r}130.0_{-0.13}^{+0.14} \\
(129.7-130.3)\end{array}$ & $156.5_{-0.39}^{+0.40}$ & $56.8_{-0.12}^{+0.13}$ & $0.985_{-0.012}^{+0.015}$ \\
\hline
\end{tabular}

Notes. Values represent the median and $68.3 \%$ credible interval of each marginalized posterior. $95.4 \%$ credible intervals are listed in parentheses.

${ }^{a}$ Here $\omega$ amd $\Omega$ are defined on the interval $[0,2 \pi)$.

$\mathrm{b}$ Time of periastron passage is expressed as a fraction of the orbital period after MJD $=0$.

determine a spectral type of M9.25 and infer a mass of 8-21 $M_{\text {Jup. }}$ Ginski et al. (2014) examined astrometry for this system, but did not find signs of orbital motion at that time. For our orbit fit we adopt a host mass of $0.64 \pm 0.04 M_{\odot}$ from Kraus \& Hillenbrand (2009), a total system mass of $0.65 \pm 0.04 M_{\odot}$, and a parallax of $7.388 \pm 0.069$ mas from Gaia DR2.

Our new observations from 2018 add to extensive published astrometry for this system, dating back to 1999 . However, these published values are highly discrepant-especially among the P.A. measurements-most likely indicating significant instrument-to-instrument calibration errors or underestimated uncertainties. This is evidenced by the excess astrometric jitter needed to bring the $\chi_{\nu}^{2}$ value for a linear fit to unity: 4.9 mas for the separations and 0.74 for the P.A.s. We find modest evidence for slight motion away from the host star at a rate of $0.5 \mathrm{mas} \mathrm{yr}^{-1}$, but no signs of any change in P.A. over time. Nevertheless, these data offer some broad constraints on the orbital properties of DH Tau B (Figure 10 and Appendix B). The semimajor axis is $330_{-160}^{+90}$ au and the orbital period is $7500_{-4800}^{+3200}$ yr $(2 \sigma$ credible interval: $2400-60,000 \mathrm{yr})$. The eccentricity is unconstrained.

\subsection{0. $2 M 1559+4403 B$}

2MASS J15594729+4403595 B is a widely separated (5". 6; $250 \mathrm{au}$ ) brown dwarf companion to a young M1.5 star and was first identified by Janson et al. (2012). Bowler et al. (2015b) measured strong lithium absorption in the optical spectrum of the companion and found a spectral type of M7.5. The system age $(\lesssim 200 \mathrm{Myr})$ and luminosity of $2 \mathrm{M} 1559+4403$ B imply a mass of $43 \pm 9 M_{\text {Jup. The host is likely a single-lined }}$ spectroscopic binary (Bowler et al. 2015b), which is bolstered by the strong astrometric excess noise (1.3 mas) reported from the Gaia DR2 astrometric fit. For this work we adopt a host mass of $0.54 \pm 0.10 M_{\odot}$ from Muirhead et al. (2018), a total system mass of $0.58 \pm 0.10 M_{\odot}$, and the Gaia DR2 parallax of the host $(22.340 \pm 0.218$ mas) for our orbit analysis.

Janson et al. (2012), Bowler et al. (2015b), Janson et al. (2014), and Bowler et al. (2015b) presented astrometry for this companion spanning 2008 to 2014, which did not reveal signs of orbital motion. We find evidence for orbital motion toward the host star in separation with our new epoch from 2018 at a

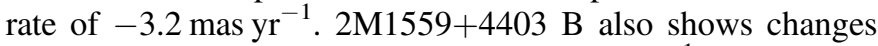
in P.A. at the $2 \sigma$ level with a rate of $-0.04 \mathrm{yr}^{-1}$. Astrometric 


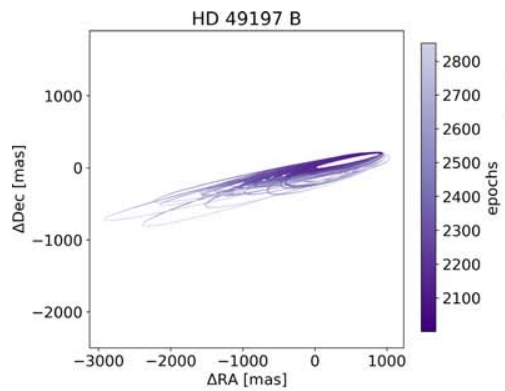

(a) HD 49197 B

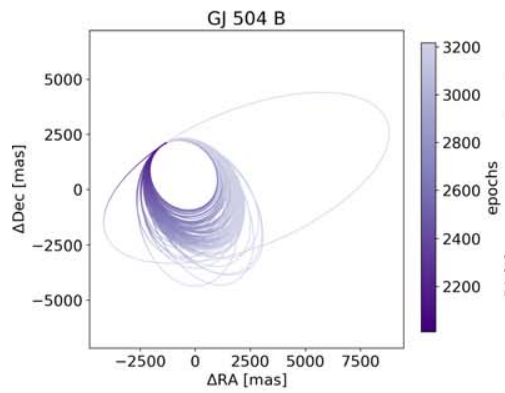

(c) GJ 504 B
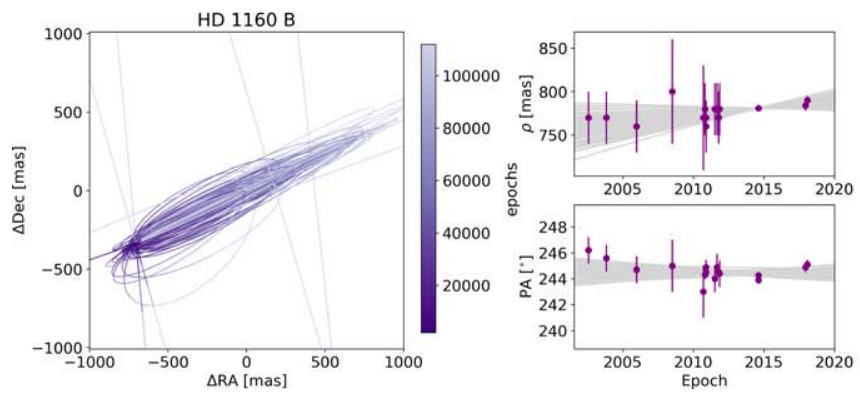

(e) HD $1160 \mathrm{~B}$
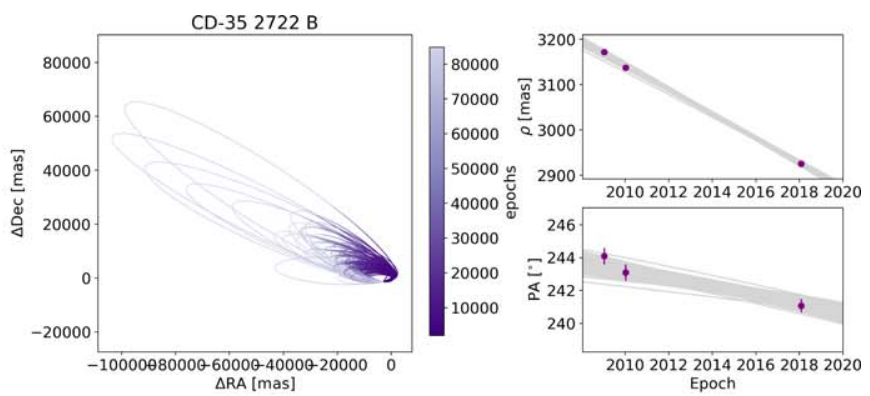

(b) CD-35 $2722 \mathrm{~B}$
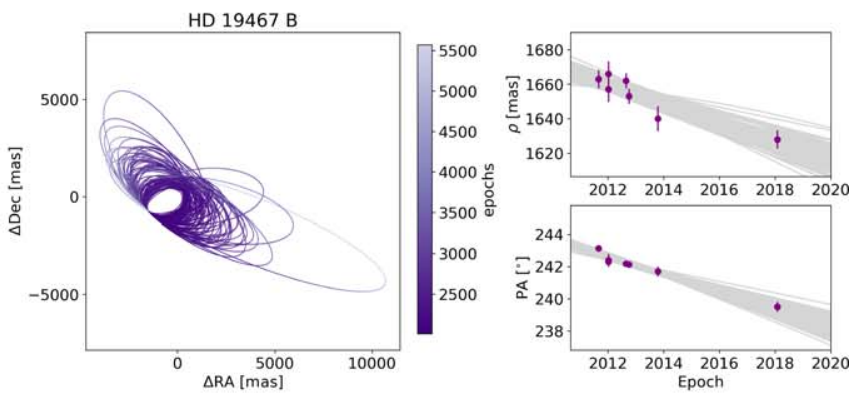

(d) HD 19467 B
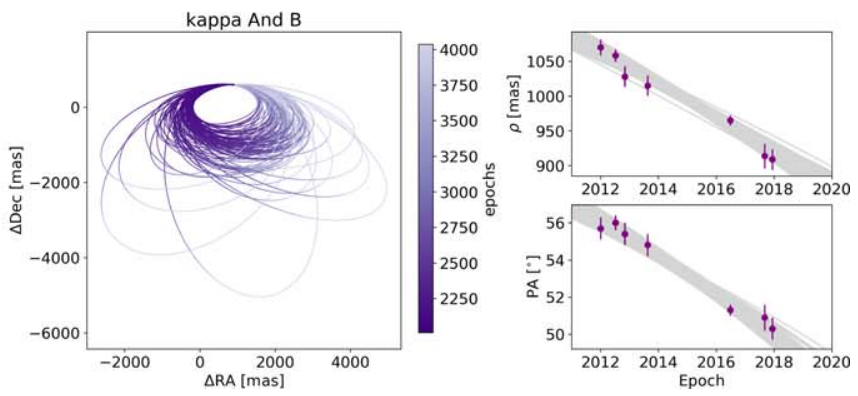

(f) $\kappa$ And B

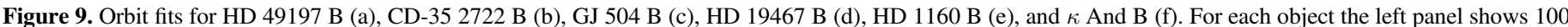

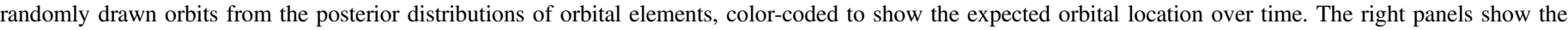

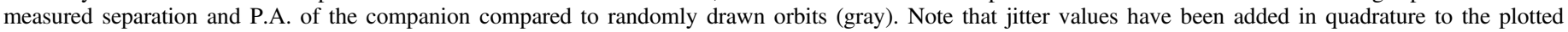
uncertainties.

jitter is required for the separation measurement uncertainties $\left(\sigma_{\mathrm{jit}, \rho}=10.5 \mathrm{mas}\right)$, but not for the P.A. errors. Our orbit constraints for this system are presented in Figure 10 and Appendix B. 2M1559+4403 B has a semimajor axis of $290_{-140}^{+80}$ au and an orbital period of $6500_{-4300}^{+2800} \mathrm{yr}(2 \sigma$ credible interval: $1800-33500 \mathrm{yr}$ ). Its eccentricity is poorly constrained, but values above $\sim 0.9$ are disfavored.

\subsection{TWA 5 B}

TWA 5 is a young $(\approx 10 \mathrm{Myr})$ triple system comprising a close $(\approx 3 \mathrm{au})$ stellar binary, TWA 5 Aab (Macintosh et al. 2001 ), and a wider brown dwarf companion located at $\approx 80 \mathrm{au}$ (Lowrance et al. 1999; Webb et al. 1999). The $6 \mathrm{yr}$ orbit of TWA 5 Aab has been well mapped over the past two decades (Konopacky et al. 2007; Köhler et al. 2013), but no orbit determination has been made for TWA 5 B. TWA 5 Aab is unresolved in our observations in 2018, but these data combined with published astrometry dating back to 1998 provide a 20 -year baseline to assess the orbit of TWA $5 \mathrm{~B}^{10}$ For our orbit analysis we adopt the dynamical mass of $0.9 \pm 0.1 M_{\odot}$ from Köhler et al. (2013) for the inner binary TWA $5 \mathrm{Aab}$, a total system mass of $0.92 \pm 0.10 M_{\odot}$, and a parallax of $20.252 \pm 0.059$ mas from Gaia DR2.

TWA $5 \mathrm{~B}$ is approaching the host pair at a rate of $-5.3 \mathrm{mas} \mathrm{yr}^{-1}$ and we measure evolution in P.A. at a rate of $-0.4 \mathrm{yr}^{-1}$ in the clockwise direction, which takes into account astrometric jitter levels of $\sigma_{\mathrm{jit}, \rho}=2.3 \mathrm{mas}$ and $\sigma_{\mathrm{jit}, \theta}=0.58$. The orbit of TWA $5 \mathrm{~B}$ about Aab is shown in Figure 10 and Appendix B, and the orbital elements are summarized in Table 3. We find a semimajor axis of $150_{-50}^{+30}$ au, a moderate eccentricity

\footnotetext{
${ }^{10}$ Note that we have assumed that the published relative astrometry for TWA $5 \mathrm{~B}$ is given with respect to the photocenter of TWA Aab. This is explicitly stated for astrometry from Köhler et al. (2013) and implied when Aab is unresolved.
} 

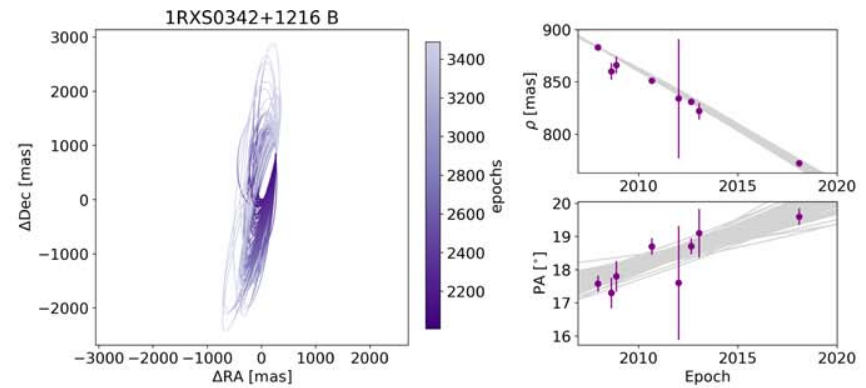

(a) $1 \mathrm{RXS} 0342+1216 \mathrm{~B}$
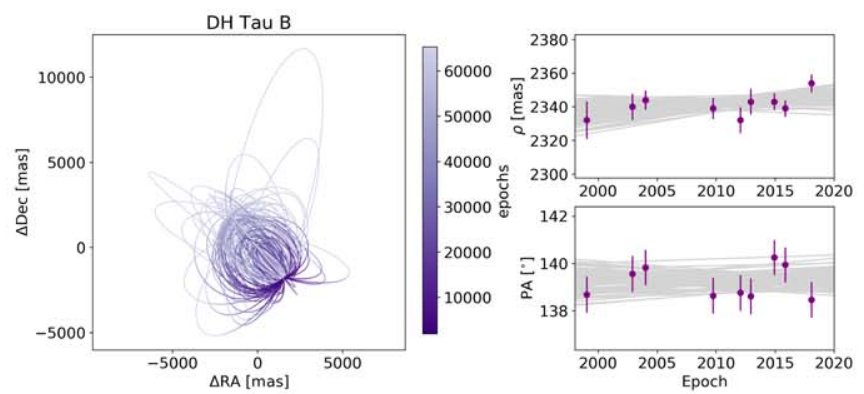

(c) DH Tau B

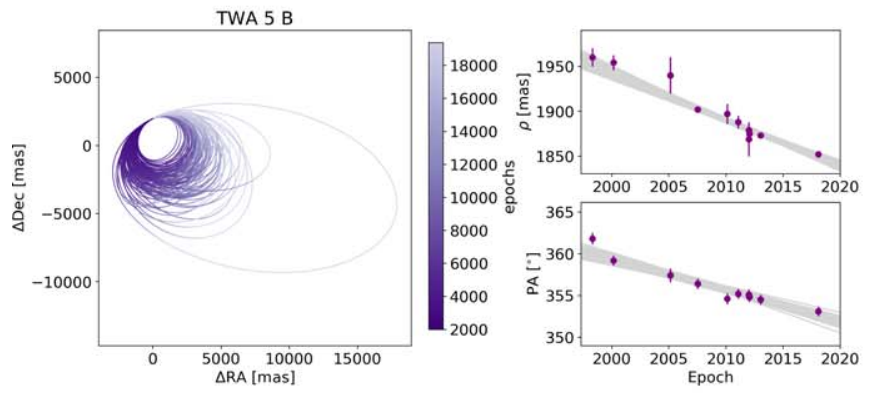

(e) TWA 5 B
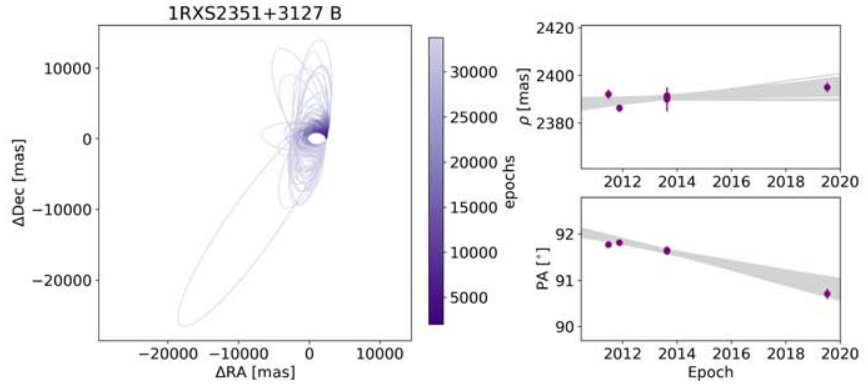

(g) 1RXS2351+3127 B

Figure 10. Orbit fits for 1RXS0342+1216 B (a), HD 23514 B (b), DH Tau B (c), 2M1559+4403 B (d), TWA 5 B (e), Ross 458 B (f), and 1RXS2351+3127 B (g) See Figure 9 for details.

of $0.43_{-0.10}^{+0.10}$, and an orbital period of $1810_{-840}^{+540} \mathrm{yr}(2 \sigma$ credible interval: $740-4400 \mathrm{yr}$ ). The orbital inclination of TWA 5 B about Aab is $151_{-13}^{+12} \circ$, which is misaligned with the measured orbital inclination of TWA 5 Aab $\left(97^{\circ} .5 \pm 0.1\right)$ from Köhler et al. (2013) at the $4 \sigma$ level. It therefore appears that the orbits of TWA 5 Aab and TWA 5 B about Aab are not coplanar.

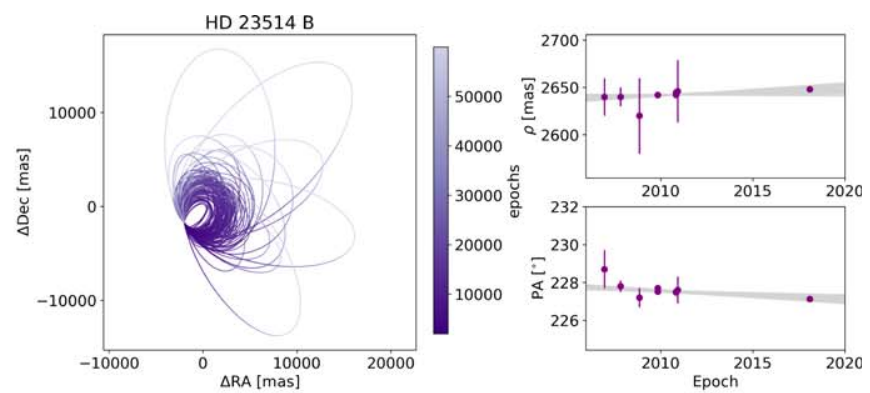

(b) HD 23514 B

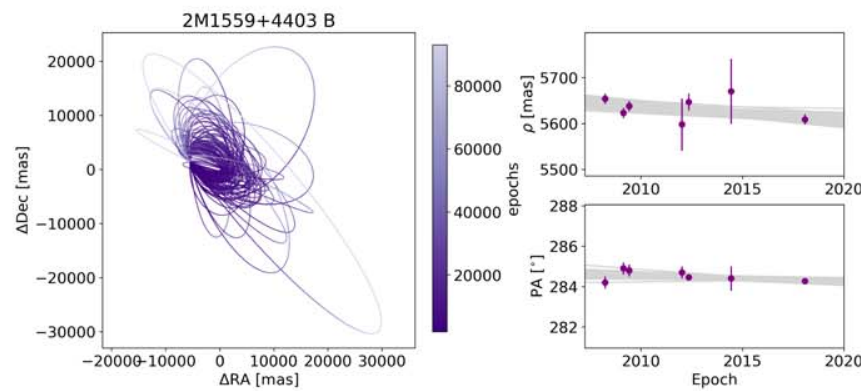

(d) $2 \mathrm{M} 1559+4403 \mathrm{~B}$
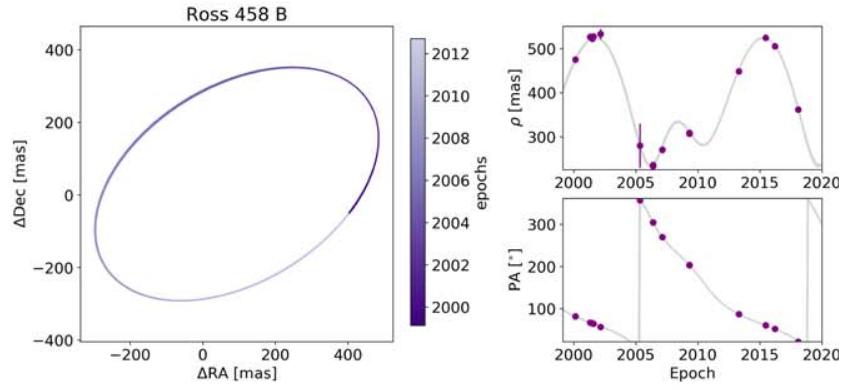

(f) Ross 458 B 
absolute magnitude of $M_{K} \approx 9.7$ (Beuzit et al. 2004), Ross 458 B resides below the hydrogen-burning limit based on the Baraffe et al. (2015) evolutionary models if its age is younger than about $300 \mathrm{Myr}$. If the age is closer to $800 \mathrm{Myr}$, then its implied mass is about $0.1 M_{\odot}$.

Heintz (1990) first detected unresolved astrometric perturbations of Ross 458 with an orbital period of $13.5 \mathrm{yr}$. This system has since been monitored with $\mathrm{AO}$ for more than a complete orbital cycle. We adopt a host star mass of $0.61 \pm 0.03 M_{\odot}$ from Neves et al. (2013), a total system mass of $0.68 \pm 0.03 M_{\odot}$, and a parallax of $86.857 \pm 0.152$ mas from Gaia DR2. Our observations in 2016 and 2018 supplement astrometry from Mann et al. (2019) and Ward-Duong et al. (2015), which together extend the astrometric coverage back to 2000. The orbit for Ross $458 \mathrm{~B}$ is therefore very well determined (see Figure 10 and Appendix B); we measure an orbital period of $13.528_{-0.014}^{+0.02}$ yr ( $2 \sigma$ credible interval: $13.49-13.56 \mathrm{yr}$ ), a semimajor axis of $4.95 \pm 0.01 \mathrm{au}$, and an orbital eccentricity of $0.242 \pm 0.001$.

\subsection{3. $1 R X S 2351+3127 B$}

1RXS J235133.3+312720 B is an early-L type substellar companion discovered as part of the PALMS survey (Bowler et al. 2012). The M2 host is a member of the $\approx 120$ Myr AB Dor moving group (Shkolnik et al. 2012; Schlieder et al. 2012; Malo et al. 2013); at this age, the inferred mass of 1RXS2351+3127 B

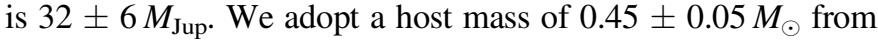
Bowler et al. (2012), a total system mass of $0.48 \pm 0.05 M_{\odot}$, and parallax of $23.218 \pm 0.052$ mas from Gaia DR2.

Bowler et al. (2015b) detected hints of orbital motion based on observations spanning 2011 to 2013. Our epoch from 2019 clearly establishes evolution in the astrometry (Figure 10). $1 \mathrm{RXS} 2351+3127 \mathrm{~B}$ is moving away from its host star at a rate of 0.8 mas yr$^{-1}$ in a clockwise direction at $-0^{\circ} 13 \mathrm{yr}^{-1}$. We find jitter terms of $\sigma_{\mathrm{jit}, \rho}=0.3 \mathrm{mas}$ and $\sigma_{\mathrm{jit}, \theta}=0.05$. 1RXS2351 $+3127 \mathrm{~B}$ has a semimajor axis of $100_{-40}^{+30}$ au and an orbital period of $1490_{-870}^{+630} \mathrm{yr}(2 \sigma$ credible interval: $570-10100 \mathrm{yr})$. Eccentricities above $\sim 0.8$ are disfavored (Appendix B).

\section{Population-level Eccentricities of Brown Dwarfs and Giant Planets}

The goal of this study is to assess whether imaged planets and brown dwarf companions on wide orbits form or dynamically evolve in similar ways by comparing their population-level eccentricity distributions. Over 100 substellar companions to stars have been discovered over the past quarter century, spanning 2-75 $M_{\text {Jup }}$. The majority of these objects are located at wide separations beyond 100 au and were largely found in seeing-limited infrared surveys such as 2MASS, PanSTARRS, and WISE. Companions located at smaller angular separations and generally within 200 au were predominantly discovered with the aid of AO. Most of these brown dwarfs and giant planets have been continuously observed since their discoveries and typically exhibit orbital motion after a few years of astrometric monitoring (Figure 11).

In this section we make use of companions undergoing orbital motion to assess the underlying eccentricity distributions of brown dwarf companions and giant planets at the population levels. We first describe our sample selection and experimental design for this analysis. For systems with small fractional orbital coverage, we perform new orbit fits in a uniform manner with orbitize! using published astrometry.

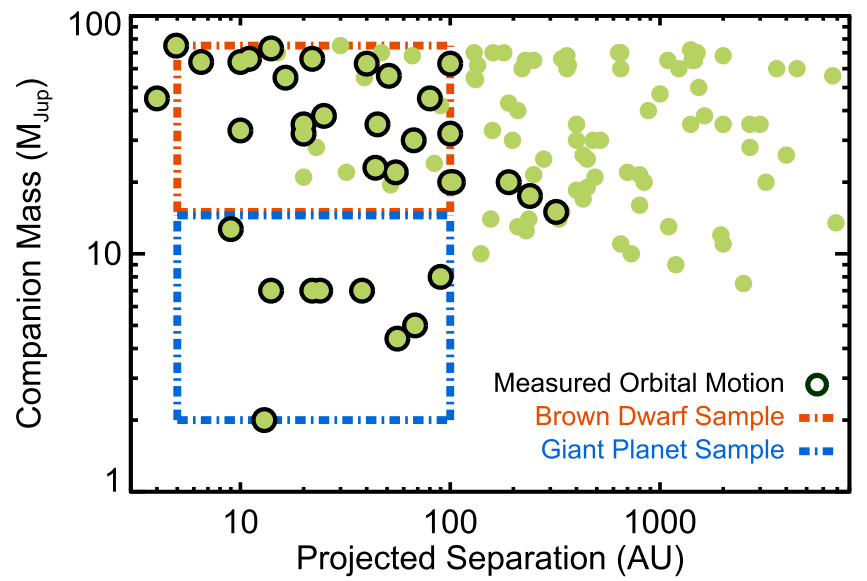

Figure 11. Sample selection for this study based on a literature compilation of 125 known companions with mass estimates below the hydrogen-burning boundary and orbiting stars. Most systems with projected separations beyond $\sim 200$ au were found in seeing-limited red-optical and infrared surveys such as 2MASS, WISE, and Pan-STARRS, whereas those closer in have typically been found with the aid of AO. Companions with measured orbital motion are highlighted in black. Among these, we isolate a sample of 27 systems with masses between 2 and $75 M_{\text {Jup }}$, separations between 5-100 au, and whose host stars appear to be single to study the underlying population-level eccentricity distribution of substellar companions. This is further divided into subsamples of 18 brown dwarfs (15-75 $\left.M_{\text {Jup }}\right)$ and 9 giant planets $\left(2-15 M_{\text {Jup }}\right)$ as shown in the red and blue boxes, respectively.

Finally, the underlying eccentricity distributions for giant planets and brown dwarfs are inferred using hierarchical Bayesian inference and compared with the eccentricity distributions of warm Jupiters and binary stars.

\subsection{Defining the Sample}

We have constructed a large (and to our knowledge complete) sample of 125 imaged substellar companions to stars. This list draws from previous catalogs of low-mass companions from Zuckerman \& Song (2009), Faherty et al. (2010), Deacon et al. (2014), and Bowler (2016), together with additional discoveries compiled from the literature. Orbital motion is detected for 36 companions based on multi-epoch astrometry, both from the literature and from our new observations in this work (Figure 11).

The following criteria are used to define our samples of brown dwarfs and giant planets, with the goal of identifying targets that are most representative of the initial dynamical conditions of this population and least likely to have been influenced by significant orbital migration as a result of dynamical encounters with a third body. Companions must have projected separations between 5 and 100 au at the time of discovery and have measured orbital motion from multi-epoch astrometry. The hosts must be stars $\left(>75 M_{\text {Jup }}\right)$, rather than brown dwarfs, to prevent biasing the samples toward binary star-like mass ratio distributions. Because the eccentricities of substellar companions can also be influenced by post-formation interactions in hierarchical triple systems (e.g., Fabrycky \& Tremaine 2007; Allen et al. 2012; Reipurth \& Mikkola 2015), we require the host stars not be known binaries. We similarly limit the sample to companions that are themselves single. Companions whose existence or characteristics are a matter of ongoing debate-in particular, LkCa15 bcd (Kraus \& Ireland 2012; Ireland \& Kraus 2014; Sallum et al. 2015; Thalmann et al. 2016; Currie et al. 2019) and HD 100546 bc 
(e.g., Quanz et al. 2013; Currie et al. 2015; Rameau et al. 2017) -are excluded from this analysis. This amounts to 27 systems that represent our full sample of directly imaged substellar companions undergoing orbital motion. Finally, we isolate two main subsamples within 5-100 au based on their masses as inferred from hot-start evolutionary models: 18 brown dwarfs spanning 15-75 $M_{\text {Jup }}$, and 9 giant planets between 2 and $15 M_{\text {Jup }}$. Characteristics of the host stars and companions are summarized Table 4.

\subsection{Uniform Orbits with Literature Astrometry}

We compiled all available astrometry of targets in our full sample with small fractional orbit coverage (see Appendix A) to reassess their orbits in a uniform manner instead of relying on orbit determinations in the literature, which can be influenced by different priors and approaches to fitting short orbital arcs. For the remaining targets with well-constrained (or moderately wellconstrained) orbits we adopt eccentricity posteriors from the literature. This includes the HR 8799 planets (bcde), for which we use the "unconstrained" eccentricity posteriors from Wang et al. (2018), Gl 229 B from Brandt et al. (2019b), and approximately Gaussian-shaped eccentricity distributions for the following five systems: $\beta$ Pic b $(e=0.24 \pm 0.06$; Dupuy et al. 2019), HD $4113 \mathrm{C}(e=0.38 \pm 0.06$; Cheetham et al. 2018), HD $4747 \mathrm{~B}(e=0.735 \pm 0.003$; Brandt et al. 2019a), Gl 758 B $(e=0.40 \pm 0.09$; Brandt et al. 2019a), and HR $7672(e=$ $0.542 \pm 0.018$; Brandt et al. 2019a). Note that because the orbits of these systems are well constrained, the choice of priors does not meaningfully influence the posteriors. Figure 12 shows the distribution of fractional orbital coverage for our sample. Most systems have traced out $<10 \%$ of their orbits. Only 11 have been imaged for over $5 \%$ of their orbital periods.

For the remaining nine systems that were not already discussed in Section 3, we apply the same Bayesian priors and fitting approach as previously discussed. Astrometric jitter for each system is assessed and implemented by identifying the amplitude of excess noise, which, when added in quadrature to quoted errors in separation and separately for P.A., results in a linear fit with a reduced $\chi^{2}$ value of 1.0 (Table 2). No additional uncertainty is added if $\chi_{\nu}^{2}$ is lower than 1.0 using the raw unadjusted errors.

Results from the orbit fits are shown in Figures 13 and 14 and summarized in Table 3. Our constraints are generally consistent with published orbits that make use of the same astrometry. Below we compare our fits with those in the literature with a particular focus on the eccentricity posteriors.

HD 984 B. This substellar companion was found by Meshkat et al. (2015) and was further characterized by Johnson-Groh et al. (2017) with Gemini/GPI as part of the GPIES survey. We adopt a stellar mass of $1.15 \pm 0.06 M_{\odot}$ from Mints \& Hekker (2017), a total system mass of $1.21 \pm 0.06 M_{\odot}$, and a parallax of $21.781 \pm 0.056$ mas from Gaia DR2 for our orbit fit. Our orbital constraints are similar to those of Johnson-Groh et al. using the same five epochs from 2012 to 2016 (Figure 13 and Appdendix B). We include a jitter term of 3.2 mas in separation, but this does not significantly alter the results. Our eccentricity posterior peaks near 0.0 with a long tail out to $e \approx 0.8$. Although this constraint is broad, circular orbits are clearly favored for this system.

51 Eri $b$. This $\approx 2 M_{\text {Jup }}$ planet was discovered by Macintosh et al. (2015) and has been reimaged several times with GPI and SPHERE since then. The M+M binary GJ 3305 AB orbits 51
Eri Ab at $\approx 2000$ au (Feigelson et al. 2006; Montet et al. 2015). A total system mass of $1.75 \pm 0.05 M_{\odot}$ (Macintosh et al. 2015) and parallax of $33.577 \pm 0.135$ mas from Gaia DR2 are fixed for our orbit fit. We make use of 11 epochs for our analysis-five from De Rosa et al. (2015), four of which were originally presented in Macintosh et al. (2015), and six from Maire et al. (2019). No additional jitter is included for this system.

Maire et al. find hints of curvature in the orbit and note a small $(\approx 1 \sigma)$ offset in P.A. between GPI and SPHERE for observations taken at similar epochs. They proceeded to add a 1.0 offset to the GPI data to reduce this difference and also removed the epoch from 2015 January from consideration because the separation is somewhat larger than for other epochs. For this study we take the astrometry at face value; no recalibrations are applied, and we consider all published astrometry. Any slight differences in astrometry for individual epochs are at the $\approx 1 \sigma$ level and are well accounted for with larger uncertainties. Indeed, linear fits to separation and P.A. over time yield $\chi_{\nu}^{2}$ values below unity (Table 2 ), suggesting that reported uncertainties may even be slightly overestimated for this system. Nevertheless, our results are very similar to those of Maire et al. (Figure 13 and Appendix B); we find a significant eccentricity for 51 Eri b of $e=0.50_{-0.08}^{+0.11}$, in good agreement with their median value of $e=0.45$ and a $68 \%$ credible interval of $e=0.30-0.55$.

$H R 2562$ B. Konopacky et al. (2016) presented four epochs of HR 2562 B with GPI in their discovery paper. They confirmed that the companion shares a common proper motion with the host star and found that its P.A. and the orientation of the disk are consistent with a nested orbit inside the disk. Maire et al. (2018) added six additional measurements over three epochs in 2016 and 2017 with SPHERE. They found orbital motion and confirmed that the orbital plane of HR 2562 B may be aligned with the debris disk. They also identified a wide range of eccentricities when all the observations were used, but values of $e \lesssim 0.3$ were preferred when a prior imposed by the debris disk was included.

Our orbit fit using a stellar mass of $1.37 \pm 0.02 M_{\odot}$ (Mesa et al. 2018), a total system mass of $1.40 \pm 0.02 M_{\odot}$, and Gaia DR2 parallax of $29.377 \pm 0.041$ mas is broadly consistent with the results from Maire et al. All eccentricities are allowed (the $2 \sigma$ credible interval spans $e=0.037-1.0$; see Figure 13 and Appendix B). We also find that HR $2562 \mathrm{~B}$ is on a nearly edgeon orbit, which is apparent from the large radial motion of HR 2562 B with respect to its host star, whereas the P.A. is nearly unchanging. Note that no additional jitter is included in our orbit fit for this system. We do not incorporate additional constraints on orbital solutions from the debris disk to avoid having to make assumptions about disk-planet coplanarity for HR 2562 and other systems with disks in this study.

$H R 3549$ B. Limited astrometry has been published for the substellar companion HR 3549 B. Mawet et al. (2015) obtained two epochs with VLT/NaCo from 2013 and 2015, and Mesa et al. (2016) acquired two astrometric measurements taken at the same epoch in 2015 with SPHERE. Mesa et al. examined orbital constraints for HR 3549 B with and without constraints from the disk. They found that high eccentricities $(e \gtrsim 0.3)$ are preferred for uninformed priors, and orbits informed by the presence of the inner disk suggest even higher values $(e \gtrsim 0.5)$.

For this system we use a stellar mass of $2.3 \pm 0.15 M_{\odot}$ from Mawet et al. (2015), a total system mass of $2.34 \pm 0.15 M_{\odot}$, 
Table 4

Sample of Substellar Companions between 2 and $75 M_{\text {Jup }}$ and 5-100 au Exhibiting Orbital Motion

\begin{tabular}{|c|c|c|c|c|c|c|c|c|c|c|c|c|c|c|}
\hline $\begin{array}{l}\text { Target } \\
\text { Name }\end{array}$ & $\begin{array}{c}\alpha \\
(\mathrm{J} 2000.0)\end{array}$ & $\begin{array}{c}\delta \\
(\mathrm{J} 2000.0)\end{array}$ & $\begin{array}{l}\text { Host } \\
\text { SpT }\end{array}$ & $\begin{array}{l}\text { Age } \\
\text { (Myr) }\end{array}$ & $\begin{array}{c}\pi^{\mathrm{a}} \\
(\mathrm{mas})\end{array}$ & $\begin{array}{c}M_{*} \\
\left(M_{\odot}\right)\end{array}$ & $\begin{array}{l}M_{\text {comp }}{ }^{\mathrm{b}} \\
\left(M_{\text {Jup }}\right)\end{array}$ & $\begin{array}{l}M_{2} / M_{1} \\
\left(\times 10^{-2}\right)\end{array}$ & $\begin{array}{l}\text { Comp. } \\
\text { SpT }\end{array}$ & $\begin{array}{c}\text { Sep. } \\
\left({ }^{\prime \prime}\right)\end{array}$ & $\begin{array}{l}\text { Sep. } \\
\text { (au) }\end{array}$ & IWA $/ a^{\mathrm{c}}$ & $\Delta t / P^{\mathrm{d}}$ & Ref. \\
\hline HD 984 B & 001410.25 & -071156.8 & F7 & $30-200$ & $21.7806 \pm 0.0564$ & $1.15 \pm 0.06$ & 34-94 & $5.3_{-1.4}^{+2.0}$ & $\mathrm{M} 6.5 \pm 1.5$ & 0.2 & 10 & 0.26 & 0.05 & $1,2,3$ \\
\hline HD 1160 B & 001557.30 & +04 1504.0 & $\mathrm{~A} 0$ & $80-125$ & $7.9417 \pm 0.0764$ & $1.95 \pm 0.10$ & $35-90$ & $3.1_{-0.8}^{+1.0}$ & $M 5.5_{-0.5}^{+1.0}$ & 0.8 & 100 & 1.18 & 0.03 & $4,5,6,7$ \\
\hline HD $4113 \mathrm{C}$ & 004312.60 & -375857.47 & G5 & $5000_{-1700}^{+1300}$ & $23.8531 \pm 0.0536$ & $1.05 \pm 0.03$ & $66 \pm 5$ & $6.0 \pm 0.5$ & $\mathrm{~T} 9 \pm 1$ & 0.5 & 22 & 0.23 & 0.008 & 8 \\
\hline HD 4747 B & 004926.76 & -231244.9 & G9 & $3300_{-1900}^{+2300}$ & $53.1836 \pm 0.1264$ & $0.82 \pm 0.08$ & $66 \pm 3$ & $7.7 \pm 0.9$ & L9-T1 & 0.6 & 11 & 0.56 & 0.27 & $9,10,11$ \\
\hline HD 19467 B & 030718.57 & -134542.4 & G3 & $4600-10000$ & $31.2255 \pm 0.0410$ & $0.95 \pm 0.02$ & $57_{-7}^{+5}$ & $5.7 \pm 0.6$ & $\mathrm{~T} 5.5 \pm 1$ & 1.6 & 51 & 0.47 & 0.02 & 12,13 \\
\hline $1 \mathrm{RXS} 0342+1216 \mathrm{~B}$ & 034231.80 & +121622.5 & M4 & $60-300$ & $30.3075 \pm 0.0668$ & $0.20 \pm 0.05$ & $35 \pm 8$ & $17 \pm 5$ & $\mathrm{~L} 0 \pm 1$ & 0.8 & 19 & 0.12 & 0.02 & 14,15 \\
\hline 51 Eri b & 043736.13 & -022824.7 & F0 & $23 \pm 3$ & $33.5770 \pm 0.1354$ & $1.75 \pm 0.05$ & $2 \pm 1$ & $0.11 \pm 0.05$ & T4-T8 & 0.45 & 13 & 0.71 & 0.11 & $16,17,18,19$ \\
\hline$\beta$ Pic b & 054717.09 & -510359.4 & A6 & $23 \pm 3$ & $50.6231 \pm 0.3339$ & $1.84 \pm 0.05$ & $13 \pm 3$ & $0.7 \pm 0.16$ & $\mathrm{~L} 1 \pm 1$ & 0.4 & 9 & 0.33 & 0.53 & $19,20,21,22$ \\
\hline CD-35 2722 B & 060919.21 & -354931.1 & M1 & $133_{-20}^{+15}$ & $44.6346 \pm 0.0262$ & $0.40 \pm 0.05$ & $31 \pm 8$ & $7 \pm 2$ & $\mathrm{~L} 4 \pm 1$ & 3.1 & 67 & 0.08 & 0.002 & 23,24 \\
\hline Gl 229 B & 061034.62 & -215152.7 & M1 & $2000-6000$ & $173.6955 \pm 0.0457$ & $0.54 \pm 0.04$ & $72 \pm 5$ & $12.7 \pm 1.3$ & $\mathrm{~T} 7 \mathrm{p} \pm 0.5$ & 6.8 & 39 & 0.79 & 0.05 & $25,26,27,28$ \\
\hline HD 49197 B & 064921.33 & +434532.8 & F5 & 260-790 & $23.9903 \pm 0.0486$ & $1.11 \pm 0.06$ & $63_{-21}^{+13}$ & $5.4 \pm 1.4$ & $\mathrm{~L} 4 \pm 1$ & 1 & 40 & 1.6 & 0.11 & $29,30,3$ \\
\hline HR 2562 B & 065001.01 & -601456.9 & F5 & $200-750$ & $29.3767 \pm 0.0411$ & $1.37 \pm 0.02$ & $32 \pm 14$ & $2.2 \pm 1.0$ & T2-T3 & 0.6 & 20 & 0.37 & 0.01 & 31,32 \\
\hline HR 3549 B & 085303.78 & -563858.1 & $\mathrm{~A} 0$ & $100-150$ & $10.4864 \pm 0.0898$ & $2.3_{-0.1}^{+0.2}$ & $40-50$ & $1.9 \pm 0.2$ & M9-L0 & 0.9 & 80 & 0.46 & 0.005 & 33,34 \\
\hline $\begin{array}{l}\text { HD } 95086 \mathrm{~b} \\
\text { GJ } 504 \mathrm{~B}^{\mathrm{f}}\end{array}$ & $\begin{array}{l}105703.01 \\
131646.52\end{array}$ & $\begin{array}{l}-684002.4 \\
+092526.9\end{array}$ & $\begin{array}{l}\text { A8 } \\
\text { G00 }\end{array}$ & $\begin{array}{c}17 \pm 4 \\
4000 \pm 1800\end{array}$ & $\begin{array}{l}11.5684 \pm 0.0325 \\
57.0186 \pm 0.2524\end{array}$ & $\begin{array}{l}1.7 \pm 0.1^{\mathrm{e}} \\
1.10-1.25\end{array}$ & $\begin{array}{c}4.4 \pm 0.8 \\
23^{+10}\end{array}$ & $\begin{array}{c}0.25 \pm 0.05 \\
1.8 \pm 0.8\end{array}$ & $\begin{array}{c}\text { L1-T3 } \\
\text { T8-T9.5 }\end{array}$ & $\begin{array}{l}0.6 \\
2.5\end{array}$ & $\begin{array}{l}52 \\
44\end{array}$ & $\begin{array}{l}0.79 \\
0.79\end{array}$ & $\begin{array}{l}0.02 \\
0.03\end{array}$ & $\begin{array}{c}35,36,37 \\
38,39\end{array}$ \\
\hline HIP 65426 b & 132436.09 & -513016.0 & $\mathrm{~A} 2$ & $14 \pm 4$ & $\begin{array}{r}5.0100 \pm 0.2524 \\
9.1566 \pm 0.0626\end{array}$ & $\begin{array}{c}1.10-1.25 \\
1.96 \pm 0.04\end{array}$ & $\begin{array}{l}8 \\
8 \pm 1\end{array}$ & $0.39 \pm 0.05$ & $\begin{array}{c}10-19.5 \\
\text { mid-L }\end{array}$ & 0.8 & 90 & 0.36 & $\begin{array}{l}0.03 \\
0.003\end{array}$ & $\begin{array}{l}30,59 \\
40,41\end{array}$ \\
\hline PDS $70 \mathrm{~b}$ & 140810.15 & -412352.57 & $\mathrm{~K} 7$ & $5 \pm 1$ & $8.8159 \pm 0.0405$ & $0.76 \pm 0.02$ & $4-10$ & $0.9 \pm 0.3$ & $\mathrm{~L}:$ & 0.2 & 22 & 0.76 & 0.05 & 42,43 \\
\hline PZ Tel B & 185305.88 & -501049.9 & K0 & $23 \pm 3$ & $21.2186 \pm 0.0602$ & $1.25_{-0.20}^{+0.05}$ & $38-72$ & $4.2_{-0.8}^{+1.0}$ & $\mathrm{M} 7 \pm 1$ & 0.3 & 17 & 0.52 & 0.07 & $44,45,19,5$ \\
\hline Gl 758 B & 192334.01 & +331319.1 & G8 & $6000-10000$ & $64.0623 \pm 0.0218$ & $0.83 \pm 0.11$ & $38.1_{-1.5}^{+1.7}$ & $4.4 \pm 0.6$ & T7-T8 & 1.8 & 25 & 0.26 & 0.04 & $\begin{array}{c}46,47,48 \\
49,11\end{array}$ \\
\hline HR 7672 B & 200406.22 & +170412.6 & G0 & $3000-5000$ & $56.4256 \pm 0.0690$ & $0.96 \pm 0.05$ & $72.7 \pm 0.8$ & $7.2 \pm 0.4$ & $\mathrm{~L} 4.5 \pm 1.5$ & 0.8 & 14 & 0.63 & 0.11 & $50,51,11$ \\
\hline HR 8799 b & 230728.72 & +210803.3 & A5 & $40 \pm 5$ & $24.2175 \pm 0.0881$ & $1.48 \pm 0.05$ & $5 \pm 1$ & $0.32 \pm 0.07$ & $\mathrm{~L} / \mathrm{Tpec}$ & 1.7 & 68 & 0.48 & 0.03 & $52,53,54,55$ \\
\hline HR 8799c & 230728.72 & +210803.3 & A5 & $40 \pm 5$ & $24.2175 \pm 0.0881$ & $1.48 \pm 0.05$ & $7 \pm 1$ & $0.45 \pm 0.07$ & $\mathrm{~L} / \mathrm{Tpec}$ & 0.95 & 38 & 0.90 & 0.08 & $52,53,54,55$ \\
\hline HR $8799 \mathrm{~d}$ & 230728.72 & +210803.3 & A5 & $40 \pm 5$ & $24.2175 \pm 0.0881$ & $1.48 \pm 0.05$ & $7 \pm 1$ & $0.45 \pm 0.07$ & L6-L8pec & 0.6 & 24 & 0.75 & 0.16 & $52,53,54,55$ \\
\hline HR 8799 e & 230728.72 & +210803.3 & A5 & $40 \pm 5$ & $24.2175 \pm 0.0881$ & $1.48 \pm 0.05$ & $7 \pm 1$ & $0.45 \pm 0.07$ & L6-L8pec & 0.4 & 14 & 0.54 & 0.13 & $\begin{array}{c}52,53,54 \\
55,56\end{array}$ \\
\hline HD 206893 B & 214521.90 & -124700.1 & F5 & $250_{-200}^{+450}$ & $24.5062 \pm 0.0639$ & $1.32 \pm 0.02$ & $15-50$ & $2.3 \pm 0.9$ & L3-L5pec & 0.3 & 10 & 0.72 & 0.10 & 57,58 \\
\hline$\kappa$ And B & 234024.51 & +442002.2 & B9 & $50_{-40}^{+30}$ & $19.9751 \pm 0.3418$ & $2.8 \pm 0.1$ & $22 \pm 9$ & $0.8 \pm 0.3$ & L0-L1 & 1.1 & 55 & 0.41 & 0.01 & $59,60,61$ \\
\hline 1RXS2351+3127 B & 235133.66 & +312722.9 & M2 & $133_{-20}^{+15}$ & $23.2183 \pm 0.0524$ & $0.45 \pm 0.05$ & $32 \pm 6$ & $6.8 \pm 1.5$ & $\mathrm{~L} 0 \pm 1$ & 2.4 & 100 & 0.26 & 0.005 & 62,24 \\
\hline
\end{tabular}

Notes.

${ }^{\text {a }}$ Parallaxes from Gaia DR2

${ }^{\mathrm{b}}$ Assumes hot-start cooling history.

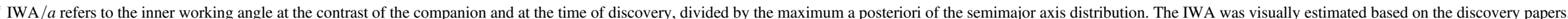

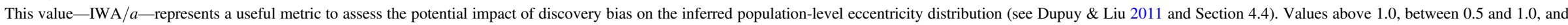
below 0.5 indicate strong, moderate, and minimal discovery bias, respectively.

${ }^{\mathrm{d}}$ Fractional orbital coverage, computed from the time baseline over which this companion has been imaged $(\Delta t)$ and the best-fit orbital period $(P)$.

${ }^{\mathrm{e}}$ Uncertainty in the host mass is approximated from multiple assessments in the literature (Meshkat et al. 2013; Chen et al. 2014).

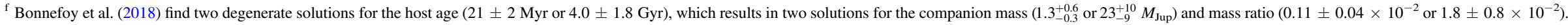
For this study we adopt the older age and correspondingly higher companion mass.

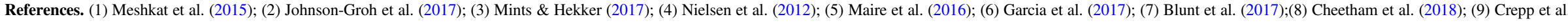

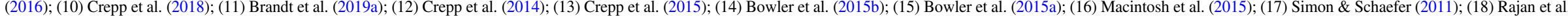

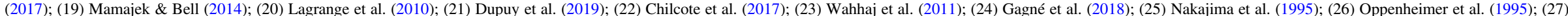

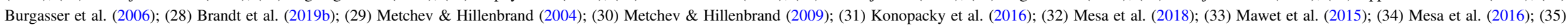

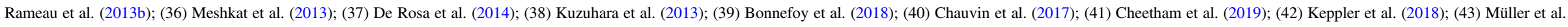

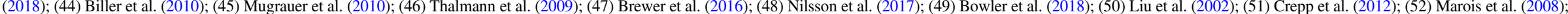

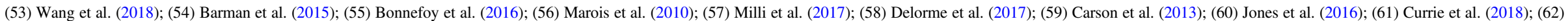
Bowler et al. (2012) 


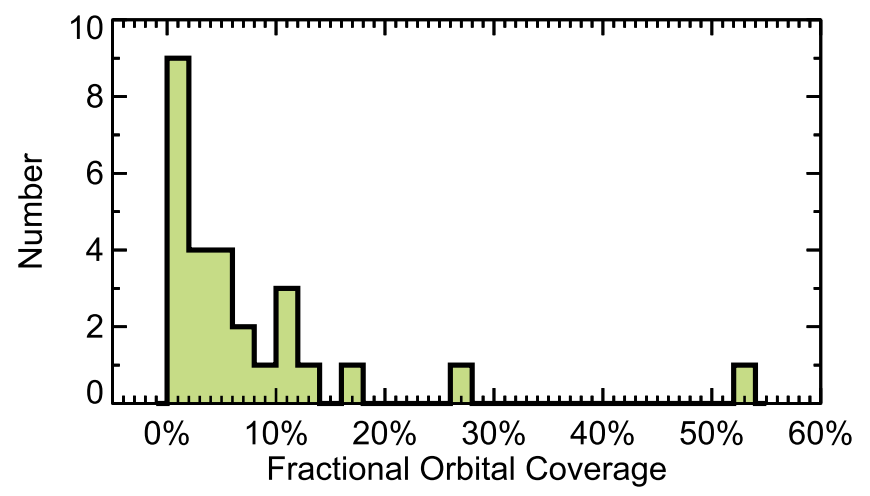

Figure 12. Fractional orbital coverage for the 27 systems in our final sample. These are determined using the first and latest published epochs of astrometry along with periods from this paper, when available, or the most recent orbit fit in literature. Most systems have only completed a few percent of their orbits from the time they were discovered to the latest observation. $\beta$ Pic $\mathrm{b}$ has the highest fractional coverage at $53 \%$, followed by HD $4747 \mathrm{~B}$ at $27 \%$.

and a parallax of $10.486 \pm 0.090$ mas from Gaia DR2. No additional jitter is added to the published astrometry. Our orbit fit is shown in Figure 13 and Appendix B. Our results disagree with the eccentricity distribution from Mesa et al.; we find that all eccentricities are possible, and that the highest eccentricities above $e \approx 0.9$ are least preferred (the $2 \sigma$ credible interval is $0.0-0.88)$. The origin of this discrepancy is not immediately obvious, but may arise from the difference between the Bayesian orbit fitting of orbitize! compared to the leastsquares Monte Carlo technique.

HD $95086 b$. HD $95086 \mathrm{~b}$ was identified by Rameau et al. (2013b) and confirmed by Rameau et al. (2013a). This companion has been continuously monitored with GPI (Rameau et al. 2016) and SPHERE (Chauvin et al. 2018) since its discovery. Both of these studies found that the companion's modest orbital motion points to lower eccentricities $(e \lesssim 0.5)$, with the most likely values being near zero.

We adopt a stellar (and total system) mass of $1.7 \pm$ $0.1 M_{\odot}$ (see Table 4) and a parallax of $11.568 \pm 0.033$ mas from Gaia DR2. No additional jitter is needed for this companion. Our results using all 20 epochs of published astrometry (Figure 13 and Appendix B) are in good agreement with those of Rameau et al. and Chauvin et al.; our $2 \sigma$ credible interval for the eccentricity is $0.0-0.48$, with low values near zero being preferred.

HIP 65426 b. Chauvin et al. (2017) discovered this longperiod planet with SPHERE and demonstrated that this object shares a common proper motion with its host star, but no orbital motion was detected. Cheetham et al. (2019) presented follow-up multi-epoch astrometry with VLT/NaCo and SPHERE that enabled them to carry out the first orbital analysis for this system. They found that the eccentricity is essentially unconstrained and is dominated by the choice in prior rather than the likelihood (the data).

Our results are shown in Figure 14 and Appendix B. We adopt a stellar mass of $1.96 \pm 0.04 M_{\odot}$ (Chauvin et al. 2017), a total system mass of $1.97 \pm 0.04 M_{\odot}$, and parallax of $9.157 \pm 0.063$ mas from Gaia DR2. We find similar results as Cheetham et al. using a modest addition of 0.12 of jitter in P.A. (but no adjustment to the uncertainties in separation): the eccentricity distribution is essentially unconstrained, and our $2 \sigma$ credible spans 0.03 to 0.97 as a result of the sparse orbital coverage for this system.
PDS $70 \mathrm{~b}$. This young planet was found by Keppler et al. (2018) nested in the transition disk of its host star using NICI, $\mathrm{NaCo}$, and SPHERE as part of the SHINE survey. Müller et al. (2018) presented additional observations with SPHERE and carried out preliminary orbit constraints, finding an eccentricity posterior that peaks near $e=0.0$ with a tail to a value of about 0.6. Additional astrometry was published by Wagner et al. (2018) using Magellan/MagAO, Christiaens et al. (2019) using VLT/SINFONI, and Haffert et al. (2019) using VLT/MUSE.

We adopt a stellar host mass of $0.76 \pm 0.02 M_{\odot}$ from Müller et al. (2018), a total system mass of $0.77 \pm 0.02 M_{\odot}$, and a parallax of $8.816 \pm 0.041$ mas from Gaia DR2. Our orbit fit using all 14 published epochs is shown in Figure 14 and Appendix B, and is summarized in Table 3. No jitter is required. Our $2 \sigma$ credible interval spans $e=0.0-0.59$, with lower values being preferred.

$P Z$ Tel B. This brown dwarf companion was independently found by Biller et al. (2010) with NICI and Mugrauer et al. (2010) with NaCo. Despite having only two epochs available, Biller et al. were able to establish a high eccentricity of $>0.6$ for this system. This has been bolstered by additional astrometry and progressively more refined orbit constraints over the past decade (Mugrauer et al. 2012; Ginski et al. 2014; Beust et al. 2016; Maire et al. 2016).

We adopt a stellar host mass of $1.25 \pm 0.10 M_{\odot}$ from Biller et al. (2010), a total system mass of $1.3 \pm 0.1 M_{\odot}$, and a parallax of $21.219 \pm 0.060$ mas from Gaia DR2 for our orbit analysis. All 26 published epochs are used for our orbit fit. There is some indication that on average, the reported uncertainties are underestimated; 3.8 mas and 0.16 of astrometric jitter are required to bring the separation and P.A. measurements in statistical agreement with a linear fit. Note that we find significant curvature in both separation and P.A. (Figure 14), suggesting we may be slightly overestimating the value of the jitter for this star. This curvature was first noted by Ginski et al. (2014), Mugrauer et al. (2012), and Maire et al. (2016). With this additional adjustment, we find that high values of eccentricity above 0.6 are strongly preferred, with the $2 \sigma$ credible interval spanning 0.74-1.0. Results for PZ Tel B can be found in Figure 14 and Appendix B.

$H D 206893$ B. Five astrometric epochs are available for this companion: two with SPHERE and $\mathrm{NaCo}$ as part of the discovery observations by Milli et al. (2017), and three additional observations with SPHERE by Delorme et al. (2017) and Grandjean et al. (2019). Delorme et al. determined initial constraints on the orbital elements for this companion and found that all eccentricities are allowed with a preference against the highest values above about 0.9 . When we consider only orbits that are coplanar with the debris disk, this collapses to a range of about $e=0.0-0.4$. More recently, Grandjean et al. (2019) combine relative astrometry, a radial acceleration, and astrometric acceleration measured between Hipparcos and Gaia to constrain the orbit and dynamical mass of HD 206893 B. They find a low eccentricity $(e \lesssim 0.4)$ and potential evidence of a second companion based on the larger-thanexpected radial acceleration.

For this study we fix the host mass at $1.32 \pm 0.02 M_{\odot}$ from Delorme et al. (2017), adopt a total system mass of $1.35 \pm 0.02 M_{\odot}$, and use a parallax of $24.506 \pm 0.064$ mas from Gaia DR2. No jitter is required in separation, but modest jitter of 0.29 is inferred for the P.A. measurements. Results from our orbit fits are presented in Figure 14 and Appendix B. 


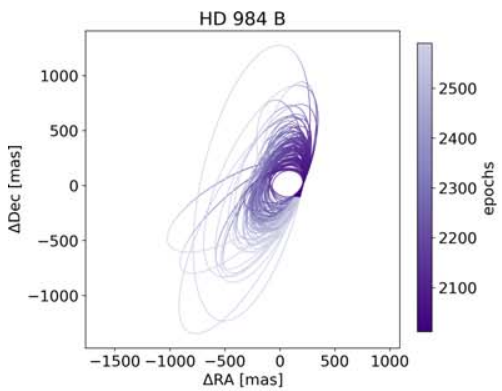

(a) HD 984 B
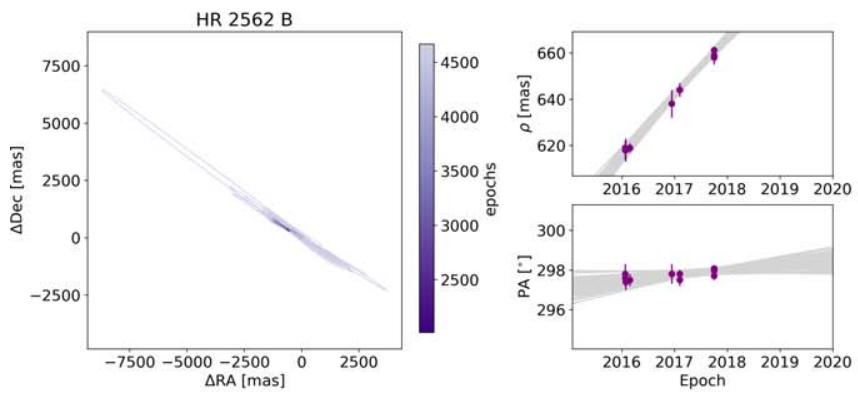

(c) HR 2562 B

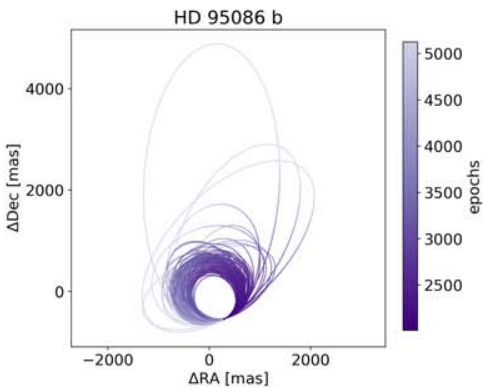

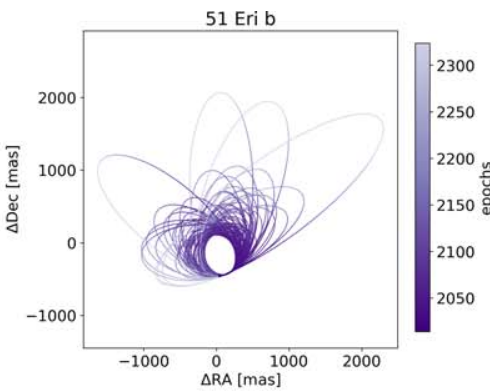

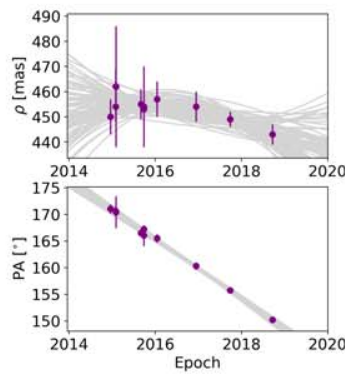

(b) 51 Eri b
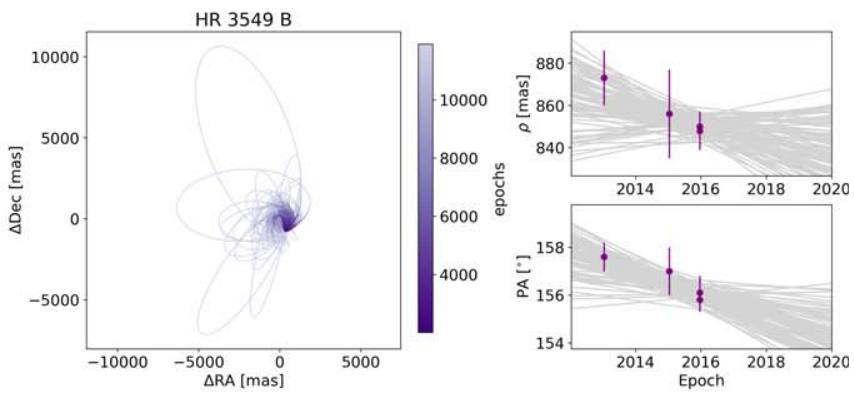

(d) HR 3549 B

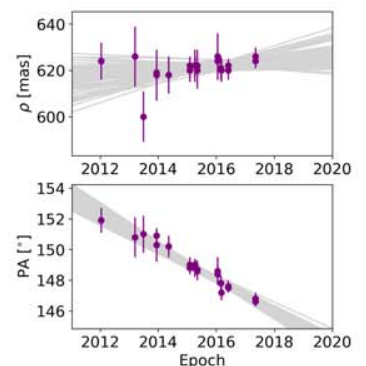

(e) HD $95086 \mathrm{~b}$

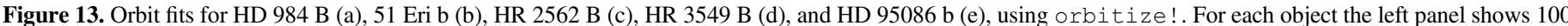

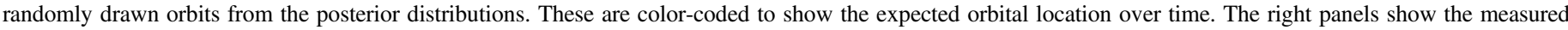
separation (top) and P.A. (bottom) of the companion compared to randomly drawn orbits from the posterior distributions.

We determine an eccentricity of $e=0.25_{-0.14}^{+0.17}$ with a $2 \sigma$ credible interval of $0.0-0.44$. Our constraints are narrower than the general unrestricted fit from Delorme et al. and are similar to the recent results from Grandjean et al. (2019), who also included relative astromety, radial velocities, and absolute astrometry of the host star from Hipparcos and Gaia in their orbit fit.

\subsection{Eccentricity Distributions with Hierarchical Bayesian Modeling}

Our goal is to determine the underlying behavior of the substellar eccentricity distribution at the population level given a sample of measured eccentricity posteriors for individual systems-some precisely determined, others broadly constrained, and most of which are asymmetric and non-Gaussian in shape (Figure 15 and Table 5). Incorporating the structure of the posterior distribution in these constraints is especially important in this study because the number of systems under consideration is relatively modest: 9 long-period planets and 18 brown dwarfs, totaling 27 substellar companions altogether.

Hierarchical Bayesian modeling offers a natural framework to incorporate this type of probabilistic information at multiple (individual and population) levels. This tool is gaining popularity in astronomy (see, e.g., Loredo 2013) and particularly within the field of exoplanets; for example, it has been used to determine exoplanet eccentricity distributions (Hogg et al. 2010; Shabram et al. 2016; Van Eylen et al. 2019), the value of $\eta_{\oplus}$ (Foreman-Mackey et al. 2014), the planet mass-radius relationship (Rogers 2015; Wolfgang et al. 2016), and host star obliquities (Morton \& Winn 2014; Campante et al. 2016).

Hierarchical Bayesian modeling enables simultaneous inference for parameters of individual systems $(\boldsymbol{\theta})$ and hyperparameters governing the underlying behavior of the population $(\boldsymbol{\Lambda})$, given the data $\boldsymbol{d}$. Here variables in bold denote vectors of multiple values, parameters, or data sets. Bayes' theorem for 


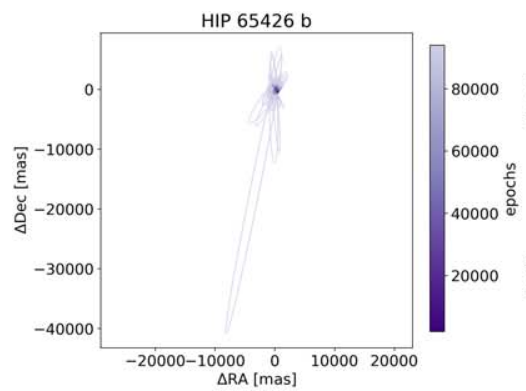

(a) HIP $65426 \mathrm{~b}$
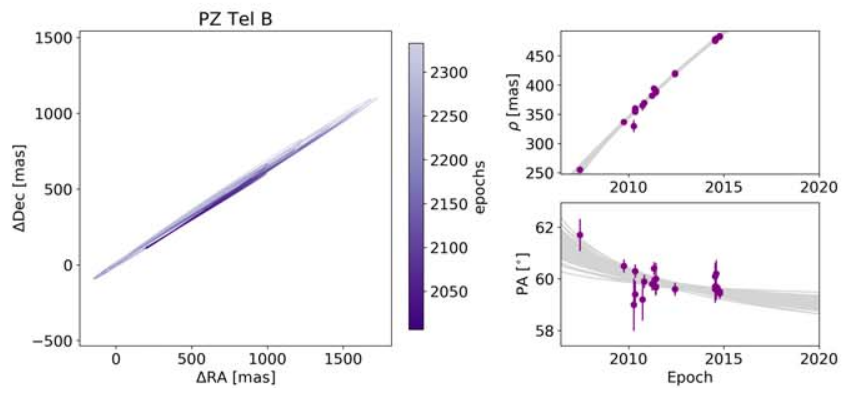

(d) PZ Tel B
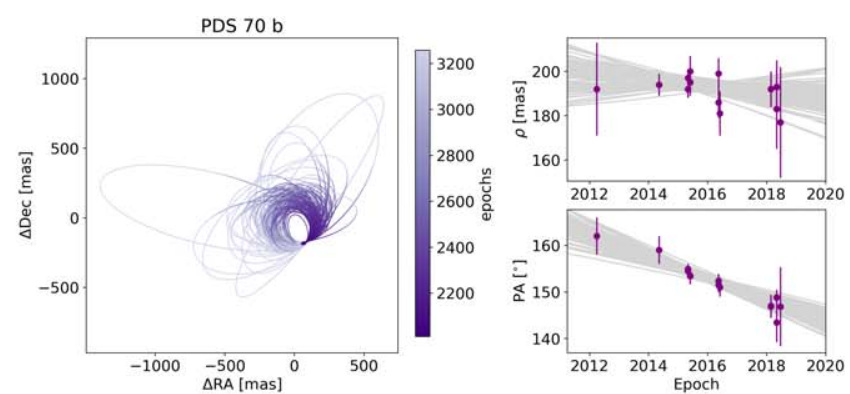

(c) PDS $70 \mathrm{~b}$
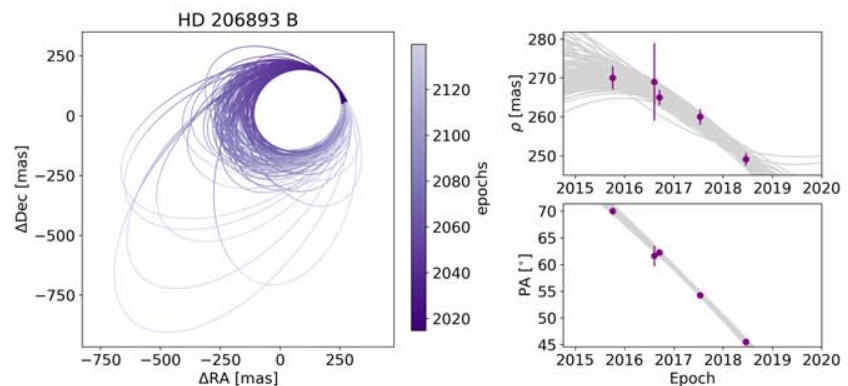

(e) HD 206893 B

Figure 14. Orbit fits for HIP 65426 b (a), PDS 70 b (b), PZ Tel B (c), and HD 206893 B (d) using orbitize!. See Figure 13 for details.

this multi-level modeling becomes

$$
p(\boldsymbol{\theta}, \boldsymbol{\Lambda} \mid \boldsymbol{d})=\frac{p(\boldsymbol{d} \mid \boldsymbol{\theta}) p(\boldsymbol{\theta} \mid \boldsymbol{\Lambda}) p(\boldsymbol{\Lambda})}{p(\boldsymbol{d})},
$$

where $p(\boldsymbol{\theta}, \boldsymbol{\Lambda} \mid \boldsymbol{d})$ is the joint posterior distribution for the individual and population parameters, $p(\boldsymbol{d} \mid \boldsymbol{\theta})$ is the likelihood function of the data, $p(\boldsymbol{\theta} \mid \boldsymbol{\Lambda})$ is the prior on the individual systems conditioned on the set of population-level hyperparameters, $p(\boldsymbol{\Lambda})$ is the hyper-prior-the prior distribution on the set of population-level parameters $\boldsymbol{\Lambda}$-and $p(\boldsymbol{d})$ is the marginalized likelihood.

For this problem, the orbit fits are carried out separately, and the individual posteriors for the eccentricity distributions (and all other orbital elements) are available. We therefore seek to constrain the hyperparameters of a parameterized model for the underlying eccentricity distribution based on observations of $N$ systems. The posterior probability distribution of $\boldsymbol{\Lambda}$ is simply

$$
p(\boldsymbol{\Lambda} \mid \boldsymbol{d}) \propto \mathcal{L}(\boldsymbol{d} \mid \boldsymbol{\Lambda}) \pi(\boldsymbol{\Lambda}) .
$$

Here $\mathcal{L}(\boldsymbol{d} \mid \boldsymbol{\Lambda})$ is the likelihood function and $\pi(\boldsymbol{\Lambda})$ is the set of priors on the hyperparameters.

Hogg et al. (2010) describe an importance sampling approach to hierarchical Bayesian modeling with a specific application to exoplanet eccentricities. We follow their method by making use of the eccentricity posterior distributions of individual systems from our orbit fits to inform the populationlevel likelihood function for parameters in the underlying eccentricity distribution, $f(e \mid \boldsymbol{\Lambda})$. In this case, the sampling approximation to the likelihood function in Equation (2) is

$$
\mathcal{L}(\boldsymbol{d} \mid \boldsymbol{\Lambda}) \approx \prod_{n=1}^{N} \frac{1}{K} \sum_{k=1}^{K} \frac{f\left(e_{n k} \mid \boldsymbol{\Lambda}\right)}{\pi\left(e_{n k}\right)},
$$

where $N$ is the number of substellar companions being considered, $K$ is the number of samples from the posterior eccentricity distribution, $e_{n k}$ is the $k$ th random draw for each eccentricity distribution $n, f\left(e_{n k} \mid \boldsymbol{\Lambda}\right)$ is the probability density of the population-level eccentricity distribution evaluated at $e_{n k}$ and conditioned on the hyperparameters $\Lambda$, and $\pi\left(e_{n k}\right)$ is the probability density of the prior probability distribution evaluated at $e_{n k}$.

We follow the approach of Hogg et al. (2010), Kipping (2013), and Van Eylen et al. (2019), by adopting a standard Beta distribution for our population-level eccentricity distribution, $f(e \mid \boldsymbol{\Lambda})$. The advantage of the standard Beta distribution functional form is that it is flexible, spans a range of $[0,1]$, and is described by only two shape parameters, $\Lambda \equiv(\alpha, \beta)$, both of which take on values $>0$ :

$$
f(e \mid \alpha, \beta)=\frac{\Gamma(\alpha+\beta)}{\Gamma(\alpha) \Gamma(\beta)} e^{\alpha-1}(1-e)^{\beta-1} .
$$

Here $\Gamma$ represents the Gamma function. The Beta distribution can capture a wide range of shapes, including uniform $(\alpha=1$, $\beta=1) ; \mathcal{U}$-shaped with a single anti-mode; unimodal with positive, negative, or no skew; $\mathcal{J}$-shaped or reverse $\mathcal{J}$-shaped; bell-shaped; and triangular. $\alpha$ governs the function's behavior at low eccentricities and $\beta$ influences its shape at high eccentricities. Low values of $\alpha$ and $\beta$ correspond to high probability densities near $e=0$ and $e=1$, while high values of these parameters correspond to low probability densities near zero. $^{11}$

\footnotetext{
${ }^{11}$ For high values of $\alpha$ and $\beta(>100)$, we use a normal approximation to the Beta distribution for computational efficiency: $B(\alpha, \beta) \approx \mathcal{N}(\mu=$ $\alpha /(\alpha+\beta), \sigma=\sqrt{(} \alpha \beta /\left((\alpha+\beta)^{2}(\alpha+\beta+1)\right)$.
} 
This simple parameterization is especially convenient for this study because it can qualitatively reproduce a wide range of potential physical outcomes of the planet formation and migration process: outward scattering, which is expected to excite eccentricities (Scharf \& Menou 2009; Veras et al. 2009); cloud fragmentation, which should result in a broad range of eccentricities; dynamically relaxed systems that follow the thermal eccentricity distribution $(f(e) \sim 2 e$; Ambartsumian 1937); and formation in a disk, in which case companions should retain nearly circular orbits if they are dynamically undisturbed.

Our primary goal is therefore to constrain the hyperparameters $\alpha$ and $\beta$ of the Beta distribution for our sample of imaged substellar companions undergoing measured orbital motion. We also examine the eccentricity distribution of other subsamples: a division of giant planets and brown dwarfs based on companion mass, a subdivision by mass ratio, imaged planets including and excluding the HR 8799 system, and divisions based on orbital separation and age. These are discussed separately in more detail below.

For each of these cases we use the Metropolis-Hastings MCMC algorithm (Metropolis et al. 1953; Hastings 1970) to sample the posterior distributions of the model parameters $\alpha$ and $\beta$. Linearly uniform hyperpriors are chosen for $\alpha$ from 0 to $1000, \beta$ from 0 to 1000 , and $\pi(e)$ from 0 to 1 . With only two parameters for the parent model and uncomplicated covariance, we use a single chain typically comprising $10^{6}$ links and $K=1000$ samples from each individual system-level posterior to explore the population-level eccentricity posterior. ${ }^{2}$ Convergence is monitored using the Gelman-Rubin (GR) statistic (Gelman \& Rubin 1992), which compares the variance within chains to the variance between chains (here our single chain divided into subchains). In all cases the GR statistic is lower than 1.1 within $10^{5}$ links, and in most cases it is lower than 1.01 , indicating that the chains are well mixed. No burn-in is warranted because of the low dimensionality of the model, and the starting points are all near the equilibrium point of the posterior distributions. We adopt normal proposal distributions with standard deviations set to avoid acceptance rates that are too high (near 1), in which case step sizes become too small to efficiently map posterior space, and too low (near 0 ) where large jumps mean that only few proposed values are accepted and convergence is slow. Most of our final acceptance rates fall between 0.3 and 0.8 .

\subsubsection{Example: Recovering the Short-period Exoplanet Eccentricity Distribution}

We carried out a series of experiments using mock data sets drawn from the actual eccentricity distribution of warm Jupiters to establish how the individual-level eccentricity measurement precision $\left(\sigma_{e}\right)$ and number of systems under consideration $(N)$ influence the ability to constrain the shape parameters $\alpha$ and $\beta$. We adopt Beta parameters $\alpha=0.867$ and $\beta=3.03$ from Kipping (2013) as underlying "truth" for this exercise and test four samples comprising 5, 10, 20, and 50 systems. For each sample we draw random eccentricity mock measurements from the population-level distribution and assign three sets of Gaussian uncertainties to these data sets, $\sigma_{e}=0.2,0.1$, and

\footnotetext{
12 The two exceptions are the "HR 8799 only" and the "Giant Planets Excluding HR 8799" cases in Section 4.3.6. For these we use $10^{7}$ links to better sample posteriors.
}

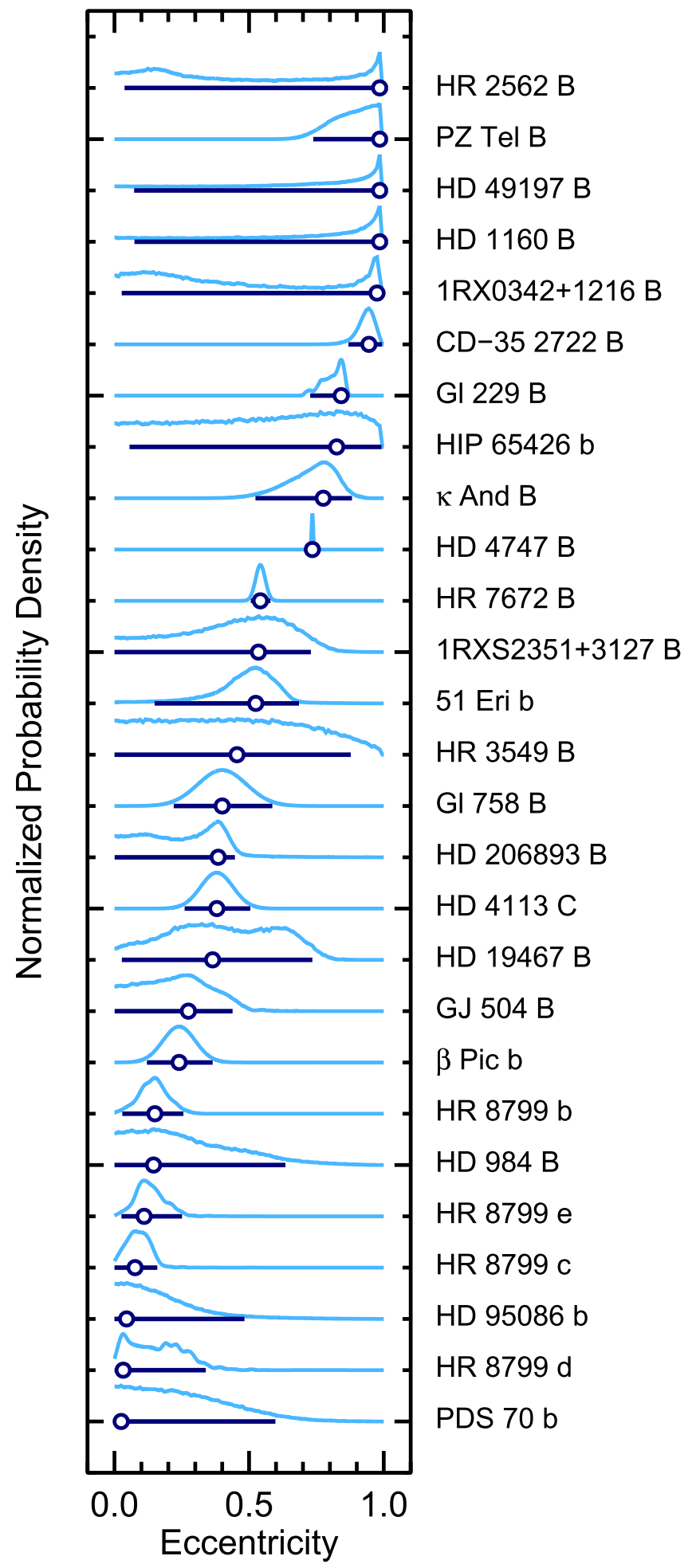

Figure 15. Posterior eccentricity distributions for the full sample of substellar companions. Objects are sorted from highest to modal value of the distribution functions, denoted with an open circle. Dark blue lines indicate $95 \%$ credible intervals. The eccentricity constraints range from well-determined to completely unconstrained, which is especially true for four of the upper five systems in this figure. The companions with the highest reliable eccentricities are PZ Tel B and CD-35 $2722 \mathrm{~B}$, which have $e>0.7$ and $e=0.94 \pm 0.03$, respectively.

0.05. These individual synthetic measurements are meant to mimic random sampling from the parent distribution and are truncated below $e=0$ and above $e=1$. 
Table 5

Summary of Individual Eccentricity Distributions

\begin{tabular}{|c|c|c|c|c|c|c|}
\hline \multirow[t]{2}{*}{ Name } & \multicolumn{4}{|c|}{$e$} & \multirow[t]{2}{*}{ Data $^{\mathrm{b}}$} & \multirow[t]{2}{*}{ Orbit Fit } \\
\hline & Median & MAP $^{\mathrm{a}}$ & $68 \%$ C.I. & 95\% C.I. & & \\
\hline HD 984 B & 0.23 & 0.15 & $0.0-0.33$ & $0.0-0.63$ & DI & This work \\
\hline HD $1160 \mathrm{~B}$ & 0.74 & 0.98 & $0.51-0.99$ & $0.067-0.99$ & DI & This work \\
\hline HD $4113 \mathrm{C}$ & 0.38 & 0.38 & $0.32-0.44$ & $0.26-0.50$ & $\mathrm{DI}+\mathrm{RV}$ & Cheetham et al. (2018) \\
\hline HD $4747 \mathrm{~B}$ & 0.73 & 0.73 & $0.73-0.74$ & $0.73-0.74$ & $\mathrm{DI}+\mathrm{RV}+$ Ast & Brandt et al. (2019a) \\
\hline HD 19467 B & 0.39 & 0.36 & $0.22-0.65$ & $0.032-0.73$ & DI & This work \\
\hline 1RX0342+1216 B & 0.34 & 0.97 & $0.0-0.59$ & $0.022-0.98$ & DI & This work \\
\hline CD-35 2722 B & 0.94 & 0.94 & $0.91-0.97$ & $0.86-0.99$ & DI & This work \\
\hline Gl 229 B & 0.82 & 0.84 & $0.79-0.86$ & $0.72-0.86$ & $\mathrm{DI}+\mathrm{RV}+$ Ast & Brandt et al. (2019b) \\
\hline HD 49197 B & 0.70 & 0.98 & $0.48-0.99$ & $0.067-0.99$ & DI & This work \\
\hline HR 2562 B & 0.42 & 0.98 & $0.0-0.69$ & $0.032-0.99$ & DI & This work \\
\hline HR 3549 B & 0.43 & 0.46 & $0.0-0.58$ & $0.0-0.87$ & DI & This work \\
\hline HD $95086 \mathrm{~b}$ & 0.14 & 0.045 & $0.0-0.21$ & $0.0-0.48$ & DI & This work \\
\hline Gl 758 B & 0.40 & 0.40 & $0.31-0.49$ & $0.22-0.58$ & $\mathrm{DI}+\mathrm{RV}+$ Ast & Brandt et al. (2019a) \\
\hline HR 7672 B & 0.54 & 0.54 & $0.52-0.56$ & $0.50-0.58$ & $\mathrm{DI}+\mathrm{RV}+$ Ast & Brandt et al. (2019a) \\
\hline HR $8799 \mathrm{~b}$ & 0.15 & 0.15 & $0.086-0.19$ & $0.031-0.25$ & DI & Wang et al. (2018) \\
\hline HR $8799 c$ & 0.088 & 0.077 & $0.041-0.13$ & $0.0040-0.16$ & DI & Wang et al. (2018) \\
\hline HR $8799 d$ & 0.15 & 0.033 & $0.011-0.22$ & $0.0-0.34$ & DI & Wang et al. (2018) \\
\hline HR 8799 e & 0.13 & 0.11 & $0.070-0.18$ & $0.024-0.25$ & DI & Wang et al. (2018) \\
\hline HD 206893 B & 0.25 & 0.39 & $0.12-0.42$ & $0.0-0.44$ & DI & This work \\
\hline$\kappa$ And $\mathrm{B}$ & 0.74 & 0.78 & $0.67-0.84$ & $0.53-0.88$ & DI & This work \\
\hline $1 \mathrm{RXS} 2351+3127 \mathrm{~B}$ & 0.46 & 0.53 & $0.28-0.70$ & $0.0010-0.73$ & DI & This work \\
\hline
\end{tabular}

Notes.

${ }^{\mathrm{a}}$ Maximum a posteriori probability.

b "DI" = relative astrometry from direct imaging; "Ast" = absolute astrometry from HGCA (Brandt 2018); "RV" = relative radial velocities.

Results of these 12 experiments are summarized in Table 6, and one example with $N=20$ and $\sigma_{e}=0.05$ is shown in Figure 16. As expected, the fidelity with which the parameters of the true distribution are recovered strongly depends on the number of measurements as well as on the precision of each measurement. As more planets are "observed" and the constraints on the orbital eccentricity improve, the median value of the posteriors for $\alpha$ and $\beta$ approach the true values and the uncertainties tend to decrease.

However, it is also clear from this exercise that randomness in the draws from the underlying eccentricity distribution can occasionally produce results that formally agree with the true parameters at the $2-3 \sigma$ level, but which have the potential to be mis- or overinterpreted. This is especially important for small samples, where stochasticity in the draws have a higher probability of producing results that qualitatively differ from the true underlying distribution. For example, in the first experiment with $N=5$ and $\sigma_{e}=0.2$, we find values of $\alpha=15.9_{-12.5}^{+7.9}$ and $\beta=31.8_{-8.1}^{+18.2}$. The resulting Beta distribution peaks at higher eccentricities than the actual distribution, which would overestimate how dynamically hot the warm Jupiter exoplanet population really is.

Although the actual values of $\alpha$ and $\beta$ are within $3 \sigma$ of the joint constraints for all 12 experiments, we conclude that the results for small sample sizes $(N \lesssim 10)$ should be interpreted as an indication of the qualitative behavior of the population. Larger sample sizes are needed to more precisely uncover the detailed shape and quantitative constraints of the underlying
Table 6

Experiments Recovering the Warm Jupiter Eccentricity Distribution

\begin{tabular}{lccc}
\hline \hline$N^{\mathrm{a}}$ & $\sigma_{e}^{\mathrm{b}}$ & $\alpha^{\mathrm{c}}$ & $\beta^{\mathrm{c}}$ \\
\hline 5 & 0.20 & $15.94_{-12.46}^{+7.86}$ & $31.75_{-8.08}^{+18.24}$ \\
5 & 0.05 & $2.03_{-1.35}^{+0.83}$ & $3.65_{-2.33}^{+1.53}$ \\
5 & 0.01 & $2.23_{-1.27}^{+0.85}$ & $14.01_{-8.35}^{+5.91}$ \\
10 & 0.20 & $8.13_{-5.39}^{+3.60}$ & $35.91_{-6.59}^{+14.05}$ \\
10 & 0.05 & $4.72_{-2.97}^{+1.83}$ & $21.24_{-12.87}^{+8.26}$ \\
10 & 0.01 & $1.97_{-0.86}^{+0.73}$ & $9.43_{-4.73}^{+3.34}$ \\
20 & 0.20 & $8.69_{-5.55}^{+3.99}$ & $31.35_{-9.05}^{+16.97}$ \\
20 & 0.05 & $1.44_{-0.54}^{+0.46}$ & $4.73_{-1.86}^{+1.51}$ \\
20 & 0.01 & $0.80_{-0.36}^{+0.25}$ & $4.89_{-2.32}^{+1.48}$ \\
50 & 0.20 & $8.60_{-3.04}^{+3.04}$ & $37.70_{-6.23}^{+12.29}$ \\
50 & 0.05 & $1.25_{-0.32}^{+0.23}$ & $3.84_{-0.97}^{+0.73}$ \\
50 & 0.01 & $0.66_{-0.15}^{+0.14}$ & $2.22_{-0.59}^{+0.46}$ \\
\hline
\end{tabular}

Notes.

${ }^{\mathrm{a}}$ Number of draws from the underlying Beta distribution $(\alpha=0.867$, $\beta=3.03$ ) from Kipping (2013).

${ }^{\mathrm{b}}$ Measurement errors for each mock realization. $\sigma_{e}$ denotes the standard deviation of the Gaussian probability distribution for the orbital eccentricity of each system.

${ }^{\mathrm{c}}$ Recovered $\alpha$ and $\beta$ shape parameters of the standard Beta distribution from hierarchical Bayesian modeling.

distribution. Even these rely on the assumption that our input model $f(e)$ is correct, however. It is certainly possible that another functional form better emulates the true population- 


\section{Recovering the RV Eccentricity Distribution}
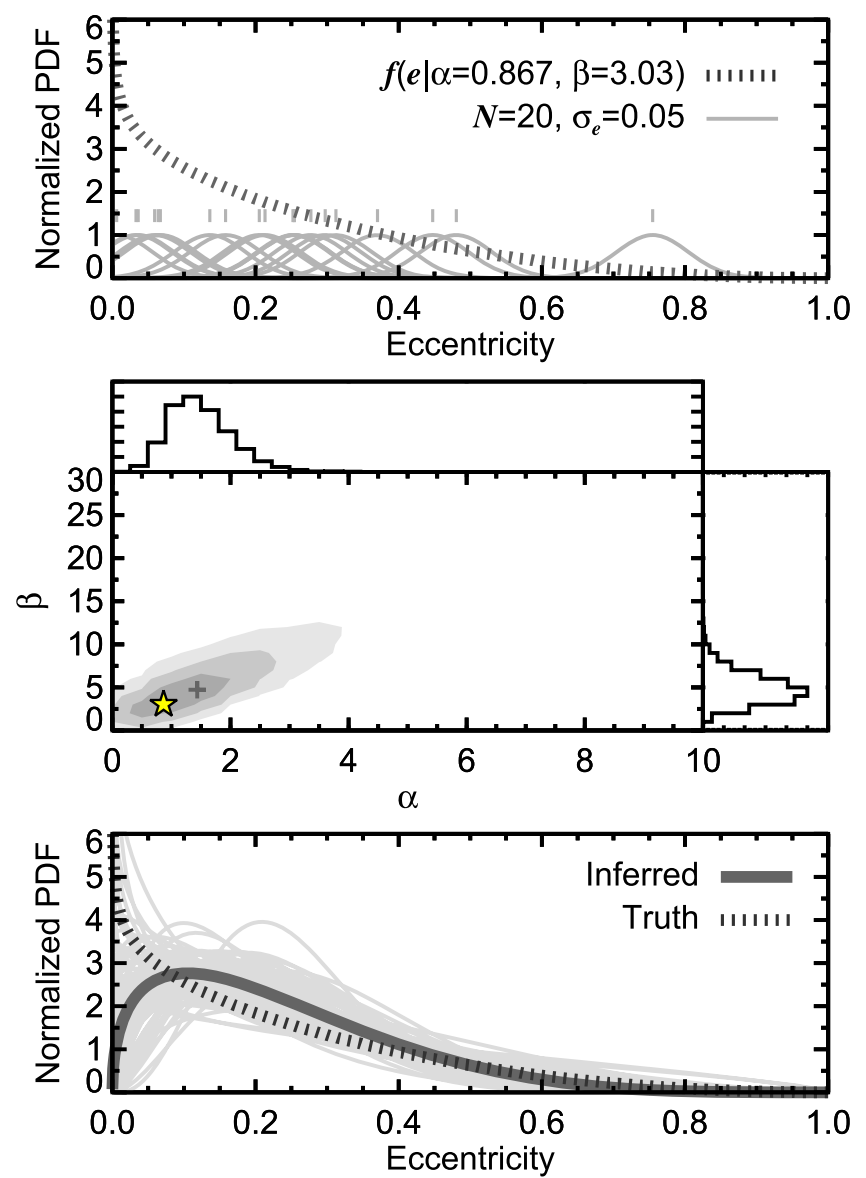

Figure 16. Example of our hierarchical Bayesian modeling approach. Here we apply this method to recover the known warm Jupiter eccentricity distribution given $N$ random mock "measurements" with various uncertainties $\left(\sigma_{e}\right)$. Top panel: The true underlying distribution (dotted curve), which is well described by a Beta distribution with $\alpha=0.867$ and $\beta=3.03$ (Kipping 2013). In this example 20 random values are drawn (thin gray curves) from the underlying parent distribution (thick dashed curve); for each of these, we assign a Gaussian uncertainty with a standard deviation of $\sigma_{e}=0.05$ (truncated at $e<0.0$ and $e>1.0)$. Middle panel: Joint distribution from MCMC sampling of the posteriors of $\alpha$ and $\beta$. Marginalized posteriors for each parameter are projected on the $x$ - and $y$-axes. Contours represent regions encompassing $1 \sigma, 2 \sigma$, and $3 \sigma$ fractions of the joint posterior distributions. The star denotes the value of the true underlying distribution-within the $1 \sigma$ contour in this example. Bottom panel: The inferred underlying distribution (thick solid curve) compared to the true distribution. Thin gray lines show 100 distributions randomly sampled from the posteriors of $\alpha$ and $\beta$. In this example the inferred distribution has captured the broad shape of the true distribution, with somewhat less fidelity (but more uncertainty) at small eccentricities. See Table 6 for results from experiments varying $N$ and $\sigma_{e}$.

level eccentricity distribution, but we avoid this type of model comparison in our orbit study because of the limited sample and the broad constraints on the eccentricity of each individual system. More targets and longer-term orbit monitoring will enable this type of model selection in the future.

To assess how readily different distributions can be distinguished from each other, we performed the same experiment for a uniform distribution as well as the warm Jupiter distribution mirrored at high eccentricities (Beta shape parameters $\alpha=3.03$ and $\beta=0.867$ ). Because we are searching for population-level differences between giant planets and brown dwarfs, these results better reflect the goals of this study. Results are summarized in Figure 17. It is clear from these tests that larger samples and better measurement precision more reliably reproduce the underlying distribution, but differences between distributions can readily be inferred from small samples. For example, even in the most pessimistic case $\left(N=5, \sigma_{e}=0.2\right)$, the warm Jupiter and high-e samples are noticeably distinct, although in this case the uniform distribution happens to resemble the warm Jupiter sample. We conclude that it is easier to determine whether two very different parent distributions are distinct from each other than it is to establish the exact shape of the underlying distribution.

\subsubsection{Eccentricity Distribution for the Full Sample}

Our results for the underlying eccentricity distribution of the full sample of 27 substellar companions spanning 5-100 au and 2-75 $M_{\text {Jup }}$ are shown in Figure 18 and summarized in Table 7. The best-fitting values of $\alpha$ and $\beta$ are $0.95_{-0.43}^{+0.41}$ and $1.30_{-0.46}^{+0.61}$, respectively, with positive covariance between the two parameters. This corresponds to an approximately flat distribution across the entire range of eccentricities. Uncertainties in the posterior are larger at the lowest and highest eccentricities and allow for some flexibility at both ends of the distribution, especially near $e=0$.

Four systems have eccentricities that are peaked near 1.0 with substantial power spanning all values: HD $49197 \mathrm{~B}$, HD $1160 \mathrm{~B}$, HR $2562 \mathrm{~B}$, and 1RXS0342+1216 B. The origin of this shape is unclear, although it may be a result of an incorrect stellar mass or perhaps underestimated astrometric uncertainties. To test whether these objects bias the results, we ran the same analysis except with uniform eccentricity distributions for these systems. The results for the full sample are nearly indistinguishable from the original case. This also holds true for all of the additional experiments we carry out in the sections below.

One of the practical implications of this result is that this flat distribution can be reliably used as a Bayesian prior for orbit fits of new substellar companions that are discovered in the future. A uniform eccentricity distribution has generally been adopted for orbit fits in the past as an uninformative prior, but this can now justifiably be used as an informed prior: in the absence of discovery bias (see Section 4.4), a newly identified substellar companion is effectively equally likely to have a low, moderate, or high eccentricity. A somewhat more precise prior would make use of the actual best-fitting Beta distribution we identify, which differs slightly from a uniform distribution.

The clearest implication of a flat posterior is that regardless of the formation or migration processes that produce this population of low-mass companions, they do not appear to imprint a strong preference for high, intermediate, or low eccentricities, at least when marginalized over other parameters such as stellar host mass, substellar mass, and system age. This analysis for the full population also implicitly assumes that the underlying eccentricity distribution of this population does not vary as a function of companion mass or separation. That is, there is no strong gradient in the orbital properties of the sample within our sample spanning 5-100 au and 2-75 $M_{\text {Jup. }}$. Below we test these assumptions by subdividing this full sample based on companion mass, mass ratio, orbital separation, and system age to determine whether there are signs of population-level changes in the eccentricities of these companions. 

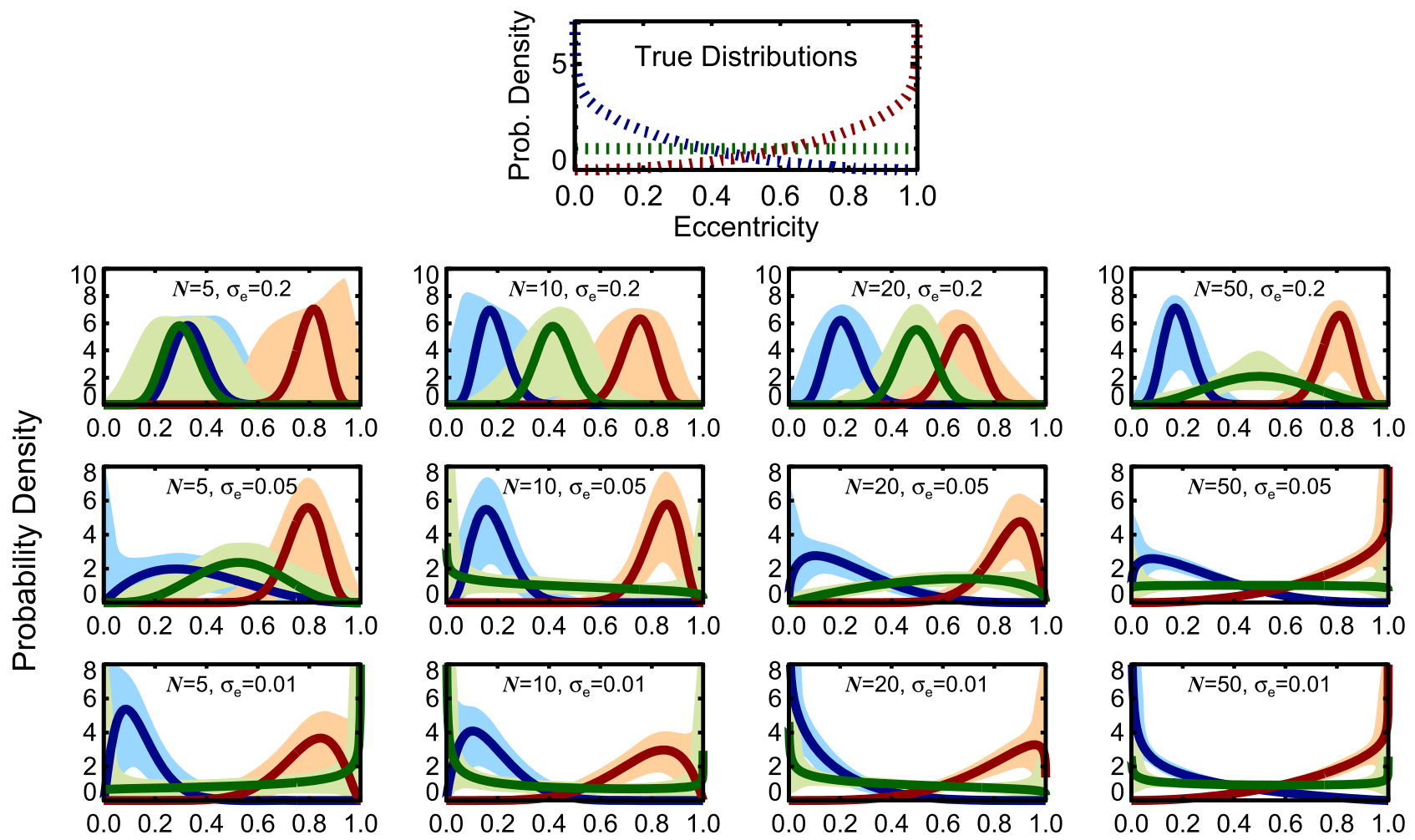

\section{Eccentricity}

Figure 17. Experiments recovering three underlying eccentricity distributions (top panel) as a function of the number of randomly drawn measurements $(N$; increasing from left to right) and Gaussian measurement uncertainty ( $\sigma_{e}$; decreasing from top to bottom). Here we test the warm Jupiter eccentricity distribution (Beta parameters $\alpha=0.867, \beta=3.03$; blue), a uniform distribution $(\alpha=1.0, \beta=1.0$; green), and the warm Jupiter distribution mirrored at high eccentricities $(\alpha=3.03, \beta=0.867$, red). The shape of the true distributions is progressively better inferred with larger samples and smaller errors. However, with precise enough measurements, these distributions can be shown to be qualitatively distinct even with small samples of $N \approx 5$. Shaded regions represent the $2 \sigma$ credible intervals from posterior draws.

Table 7

Population-level Eccentricity Distributions

\begin{tabular}{|c|c|c|c|c|c|c|}
\hline Sample & $\begin{array}{l}\text { Separation } \\
\text { Range }\end{array}$ & $\begin{array}{l}\text { Mass } \\
\text { Range }\end{array}$ & $\begin{array}{c}\text { Other } \\
\text { Constraints }\end{array}$ & $\begin{array}{l}\text { Sample } \\
\text { Size }\end{array}$ & $\begin{array}{c}\alpha \\
(1 \sigma \text { C.I. })\end{array}$ & $\begin{array}{c}\beta \\
(1 \sigma \text { C.I. })\end{array}$ \\
\hline Full Sample & $5-100$ au & $2 M_{\text {Jup }} \leqslant M_{2}<75 M_{\text {Jup }}$ & $\cdots$ & 27 & $0.950(0.520-1.36)$ & $1.30(0.840-1.91)$ \\
\hline Giant Planets & $5-100 \mathrm{au}$ & $2 M_{\text {Jup }} \leqslant M_{2}<15 M_{\text {Jup }}$ & $\cdots$ & 9 & $30.0(58.2-156)$ & $200(542-1000)$ \\
\hline Brown Dwarfs & $5-100 \mathrm{au}$ & $15 M_{\text {Jup }} \leqslant M_{2}<75 M_{\text {Jup }}$ & $\cdots$ & 18 & $2.30(1.02-3.68)$ & $1.65(0.860-2.55)$ \\
\hline High Mass Ratio & $5-100 \mathrm{au}$ & $0.01<M_{2} / M_{1}<0.2$ & $\cdots$ & 17 & $1.85(0.930-3.34)$ & $1.25(0.800-2.44)$ \\
\hline Low Mass Ratio & $5-100 \mathrm{au}$ & $0.001<M_{2} / M_{1}<0.01$ & $\cdots$ & 10 & $1.50(0.500-3.68)$ & $4.50(1.52-12.8)$ \\
\hline Close Separations & $5-30$ au & $2 M_{\text {Jup }} \leqslant M_{2}<75 M_{\text {Jup }}$ & $\ldots$ & 14 & $1.70(0.830-2.87)$ & $2.75(1.52-4.45)$ \\
\hline Wide Separations & $30-100 \mathrm{au}$ & $2 M_{\text {Jup }} \leqslant M_{2}<75 M_{\text {Jup }}$ & $\cdots$ & 13 & $0.650(0.260-1.28)$ & $0.850(0.510-1.56)$ \\
\hline Planets Excluding HR 8799 & $5-100 \mathrm{au}$ & $2 M_{\text {Jup }} \leqslant M_{2}<15 M_{\text {Jup }}$ & No HR 8799 bcde & 5 & $90.0(86.9-311)$ & $300(555-1000)$ \\
\hline Only HR 8799 & $5-100 \mathrm{au}$ & $2 M_{\text {Jup }} \leqslant M_{2}<15 M_{\text {Jup }}$ & Only HR 8799bcde & 4 & $120(43.8-127)$ & $960(570-1000)$ \\
\hline Short-Period Brown Dwarfs & $5-30 \mathrm{au}$ & $15 M_{\mathrm{Jup}} \leqslant M_{2}<75 M_{\mathrm{Jup}}$ & $\cdots$ & 9 & $3.25(1.18-8.05)$ & $3.25(1.37-7.22)$ \\
\hline Long-Period Brown Dwarfs & $30-100 \mathrm{au}$ & $15 M_{\text {Jup }} \leqslant M_{2}<75 M_{\text {Jup }}$ & $\cdots$ & 9 & $3.12(1.11-7.69)$ & $1.75(0.680-3.39)$ \\
\hline Young Brown Dwarfs & $5-100 \mathrm{au}$ & $15 M_{\text {Jup }} \leqslant M_{2}<75 M_{\text {Jup }}$ & $<1 \mathrm{Gyr}$ & 11 & $1.50(0.210-5.61)$ & $1.05(0.410-2.29)$ \\
\hline Old Brown Dwarfs & $5-100 \mathrm{au}$ & $15 M_{\text {Jup }} \leqslant M_{2}<75 M_{\text {Jup }}$ & $>1 \mathrm{Gyr}$ & 7 & $4.70(2.37-9.60)$ & $4.35(2.33-7.87)$ \\
\hline Nearby Brown Dwarfs & $5-100 \mathrm{au}$ & $15 M_{\text {Jup }} \leqslant M_{2}<75 M_{\text {Jup }}$ & $<40 \mathrm{pc}$ & 8 & $4.50(1.69-9.33)$ & $2.25(0.910-4.22)$ \\
\hline Distant Brown Dwarfs & $5-100 \mathrm{au}$ & $15 M_{\text {Jup }} \leqslant M_{2}<75 M_{\text {Jup }}$ & $>40 \mathrm{pc}$ & 10 & $1.20(0.500-7.06)$ & $1.20(0.680-6.91)$ \\
\hline Small IWA & $5-100 \mathrm{au}$ & $2 M_{\text {Jup }} \leqslant M_{2}<75 M_{\text {Jup }}$ & IWA $/ a<0.5$ & 12 & $1.00(0.650-2.37)$ & $1.20(0.880-2.97)$ \\
\hline Large IWA & $5-100 \mathrm{au}$ & $2 M_{\mathrm{Jup}} \leqslant M_{2}<75 M_{\mathrm{Jup}}$ & IWA $/ a>0.5$ & 15 & $0.600(0.430-1.46)$ & $0.800(0.770-2.25)$ \\
\hline
\end{tabular}

\subsubsection{Giant Planets and Brown Dwarf Companions}

We further subdivide our full sample of 27 substellar companions by mass to assess whether there is evidence for a difference between the population-level eccentricities of giant planets and brown dwarf companions. We adopt a dividing line of $15 M_{\text {Jup }}$ in this study, which is motivated by the approximate transition at smaller separations between the planet mass function and that of stellar or substellar companions from radial velocity samples (e.g., Udry \& Santos 2007; Schneider et al. 2011). These subsamples consist of 9 directly imaged longperiod giant planets $\left(2-15 M_{\text {Jup }}\right)$ and 18 brown dwarf companions (15-75 $\left.M_{\text {Jup }}\right)$. 
Fits to the giant planet subsample are presented in Figure 19 and Table 7. The best-fitting values are $\alpha=30$ and $\beta=200$, with $1 \sigma$ credible intervals spanning 58-156 and 540-1000, respectively. Note that the peak of this joint distribution does not fall within the marginalized $1 \sigma$ intervals because of the strong positive covariance between these parameters. Nevertheless, despite this broad range of values, the two components in each $\{\alpha, \beta\}$ pair tend to balance each other to produce a fairly narrow range for the resulting eccentricity distribution function between $e=0.05-0.25$, and with a peak value at $\bar{e}=0.13$.

However, as discussed in Section 4.3.1, we caution that interpretations of the resulting eccentricity posterior for small samples should be made with care. Here our sample size comprises nine systems, but several of the individual eccentricities are so broad that they provide little meaningful constraint at the population level. Moreover, the additional discovery of a single moderate- to high-eccentricity planet has the potential to substantially inflate this distribution. So while the resulting posterior is expected to capture the overall qualitative trend of the underlying eccentricities, our experiments in Section 4.3.1 indicate that small sample sizes can influence the detailed behavior of the reconstructed distribution, especially near the endpoints at $e=0$ or $e=1$. It is therefore unclear whether the lack of power at the lowest eccentricities reflects something genuine about this population or is perhaps just a reflection of small number statistics. New discoveries and continued orbit monitoring of these systems are needed to address this question in the future.

Results for the sample of brown dwarf companions are summarized in Figure 20. The best-fitting hyperparameters are $\alpha=2.3_{-1.3}^{+1.4}$ and $\beta=1.7_{-0.8}^{+0.9}$, with significant positive covariance between the two parameters. The eccentricity distribution for brown dwarfs peaks between $e \approx 0.6-0.9$ with a broad range between $e \approx 0.3-1.0$. If a single Beta distribution adequately describes this population, it appears that nearcircular orbits are rare, and there is an indication that the highest values above 0.9 are disfavored.

The most striking aspect of this underlying distribution is its dissimilarity with results for the giant planets. As a population, widely separated brown dwarf companions are significantly more eccentric and span a wider range of eccentricities than their lower mass counterparts. This tendency is evident in Figure 21, which shows eccentricities for individual systems as a function of companion mass. There is a noticeable dearth of planets at high eccentricities; most of the cumulative power is focused at low values. On the other hand, brown dwarfs span a wide range of eccentricities, with fewer companions on nearcircular orbits. We revisit the implications of these distinct distributions in Section 5.

To quantify the difference between these two inferred eccentricity distributions, we calculate the probability that random variables $p$ drawn from the brown dwarf distribution (BD), are greater than the giant planet (GP) distribution (e.g., Cook 2003; Raineri et al. 2014),

$$
\begin{aligned}
& P\left(p_{\mathrm{BD}}>p_{\mathrm{GP}}\right) \\
& \quad=\int_{0}^{1} f_{\mathrm{BD}}\left(e \mid \alpha_{\mathrm{BD}}, \beta_{\mathrm{BD}}\right) F_{\mathrm{GP}}\left(e \mid \alpha_{\mathrm{GP}}, \beta_{\mathrm{GP}}\right) d e,
\end{aligned}
$$

where $f_{\mathrm{BD}}\left(e \mid \alpha_{\mathrm{BD}}, \beta_{\mathrm{BD}}\right)$ is the probability distribution function for brown dwarfs and $F_{\mathrm{GP}}\left(e \mid \alpha_{\mathrm{GP}}, \beta_{\mathrm{GP}}\right)$ is the cumulative
Full Sample
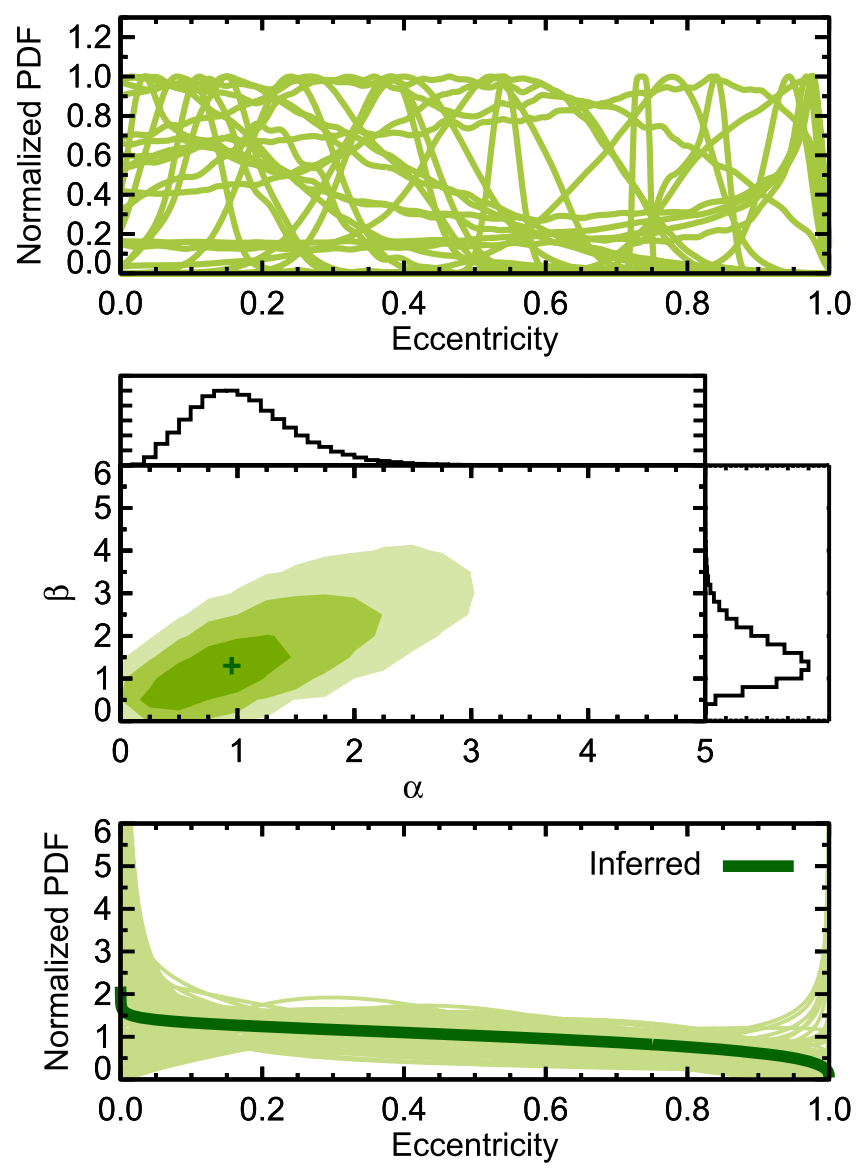

Figure 18. Same as Figure 16, but for the full sample of 27 substellar companions (2-75 $\left.M_{\text {Jup }}, 5-100 \mathrm{au}\right)$. Individual eccentricity posteriors are plotted in the top panel. The middle and bottom panels show the best-fitting $\alpha$ and $\beta$ values of the underlying Beta distribution, and the corresponding population-level posterior distribution for the full sample. The eccentricity distribution of substellar companions is approximately flat from $e=0.0-1.0$ with no significant evidence of a preference for high, intermediate, or low eccentricities.

distribution function (CDF) for giant planets. The parameterized model we adopt for the underlying population-level eccentricities is the Beta distribution. The CDF of the Beta distribution is the regularized incomplete beta function,

$$
F_{\mathrm{GP}}\left(e \mid \alpha_{\mathrm{GP}}, \beta_{\mathrm{GP}}\right)=\frac{\int_{0}^{e} t^{\alpha_{\mathrm{GP}}-1}(1-t)^{\beta_{\mathrm{GP}}-1} d t}{\int_{0}^{1} t^{\alpha_{\mathrm{GP}}-1}(1-t)^{\beta_{\mathrm{GP}}-1} d t} .
$$

Pairs of $\alpha$ and $\beta$ are randomly drawn from the joint posteriors for both the giant planet and brown dwarf MCMC results, and Equation (5) is numerically integrated for each trial. This procedure is repeated $10^{5}$ times to produce a distribution of probabilities. We find that $P\left(p_{\mathrm{BD}}>p_{\mathrm{GP}}\right)=0.979$ with a $2 \sigma$ credible interval of $0.85-1.0$. Two equivalent distributions will produce probabilities of $50 \%$, so this value of $\approx 98 \%$ points to a significant difference between these two populations.

\subsubsection{A Mass Ratio Threshold}

A single companion mass may not be a reliable threshold for distinguishing planets from brown dwarfs. Protoplanetary disk 
Giant Planets
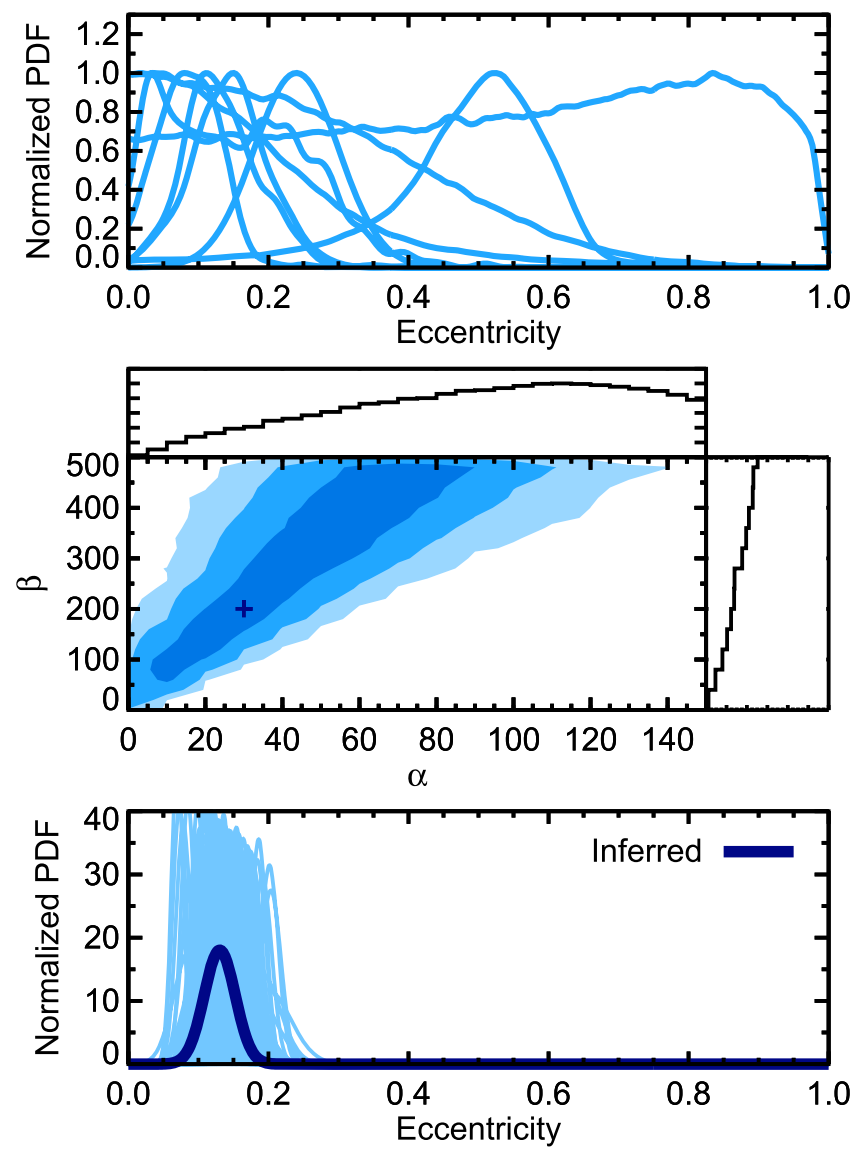

Figure 19. Same as Figure 16, but for the sample of 9 imaged planets (2-15 $\left.M_{\mathrm{Jup}}, 5-100 \mathrm{au}\right)$. The eccentricity distribution of giant planets indicates a preference for low eccentricities $(e \approx 0.05-0.25)$. Following our cautionary results for small samples in Section 4.3.1, we interpret this as a qualitative indication that long-period planets tend to have low eccentricities, not necessarily that circular orbits or moderate eccentricities are strongly disfavored.

masses correlate with host star masses (Andrews et al. 2013), so the maximum mass of a planet can be expected to also scale with stellar mass. Similarly, low-mass companions in high mass ratio systems such as 2 M1207-3932 b indicate that the companion mass is not likely to be the best criterion to distinguish giant planets from brown dwarfs. Using the companion mass ratio rather than the companion mass may be a more appropriate way to divide the full sample.

We carried out the same procedure as described above, except that we used a mass ratio of 0.01 to divide our sample into high mass ratio and low mass ratio bins. This value corresponds to a $10 M_{\text {Jup }}$ companion orbiting a Sun-like star, or a $1 M_{\text {Jup }}$ companion to a $0.1 M_{\odot}$ host star. The difference between this experiment and the prior analysis with a mass cutoff is that a single high- $e$ system- $-\kappa$ And $\mathrm{B}-$ has joined the previous giant planet sample. Results are summarized in Figure 21 and Table 7 . We measure hyperparameter values of $\alpha=1.5_{-1.0}^{+2.2}$ and $\beta=4.5_{-3.0}^{+8.3}$ for the low-mass ratio subsample and $\alpha=1.9_{-0.9}^{+1.5}$ and $\beta=1.3_{-0.5}^{+1.2}$ for the high mass ratio systems. The high mass ratio distribution is statistically indistinguishable from the brown dwarf subsample in

\section{Brown Dwarf Companions}
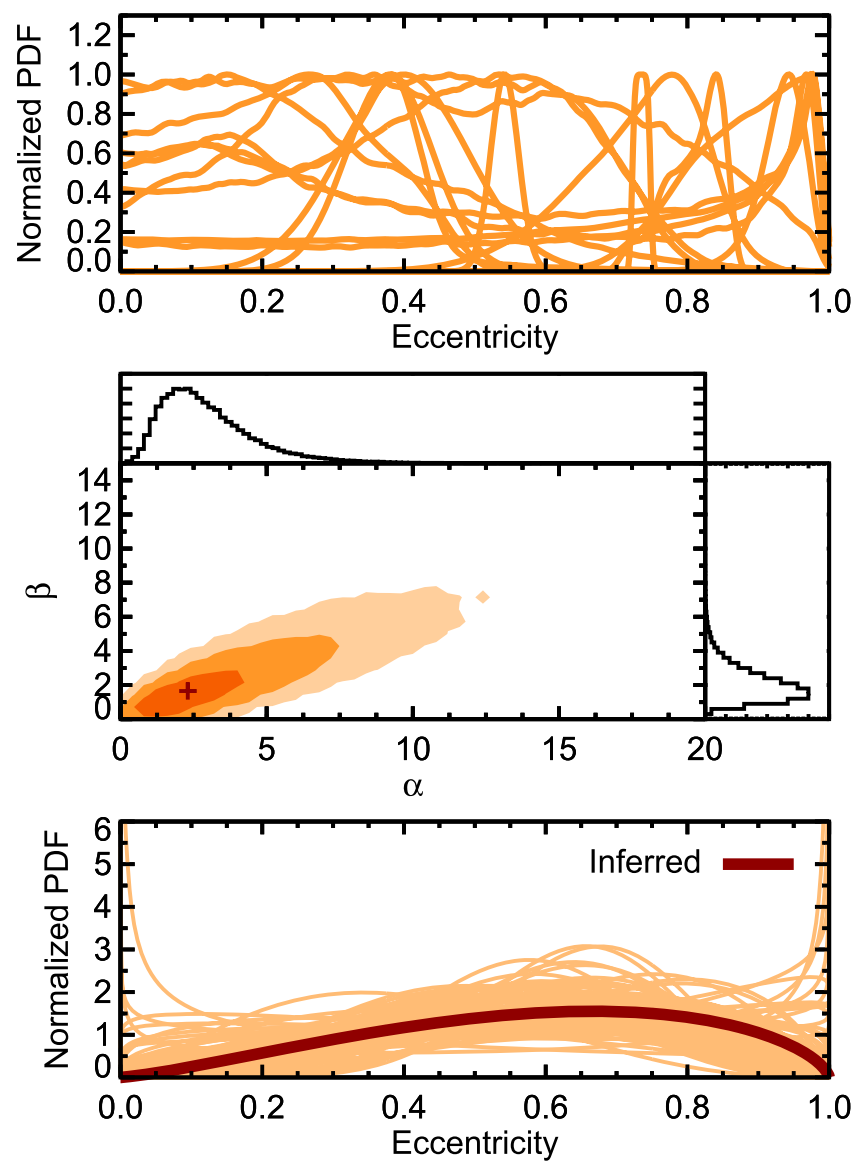

Figure 20. Same as Figure 16, but for the sample of 18 brown dwarf companions (15-75 $\left.M_{\mathrm{Jup}}, 5-100 \mathrm{au}\right)$. The eccentricity distribution of brown dwarfs indicates a broad peak toward high values, which differs substantially from population-level distribution for giant planets in Figure 19.

Section 4.3.3. The low mass ratio results are qualitatively similar to the giant planet subsample in that both generally point to low eccentricities. However, the low mass ratio distribution exhibits a broader peak and a longer tail to modest eccentricities $(e \approx 0.6)$, indicating that a substantial portion of this population is located on distinctly noncircular orbits. Using the same approach as in Section 4.3.3, we find that the high mass ratio distribution results in higher eccentricities $87.0 \%$ of the time $(2 \sigma$ credible interval: $67.8 \%-100 \%)$. This difference is substantial, suggesting dynamically distinct populations, but it is not as strong as the giant planet and brown dwarf subsamples.

This underlying distribution for low mass ratio systems bears a close resemblance to the warm Jupiter eccentricity distribution-both qualitatively, with most of the posterior power located at low eccentricities, and quantitatively with comparable values of $\alpha$ and $\beta$ (which for warm Jupiters is 0.867 and 3.03, respectively; Kipping 2013). Altogether, this indicates that the choice of mass or mass ratio to divide the parent sample does not significantly affect the interpretation of the reconstructed population-level eccentricity distribution: at wide separations, the tail end of the companion mass function appears to have dynamically distinctive properties. 

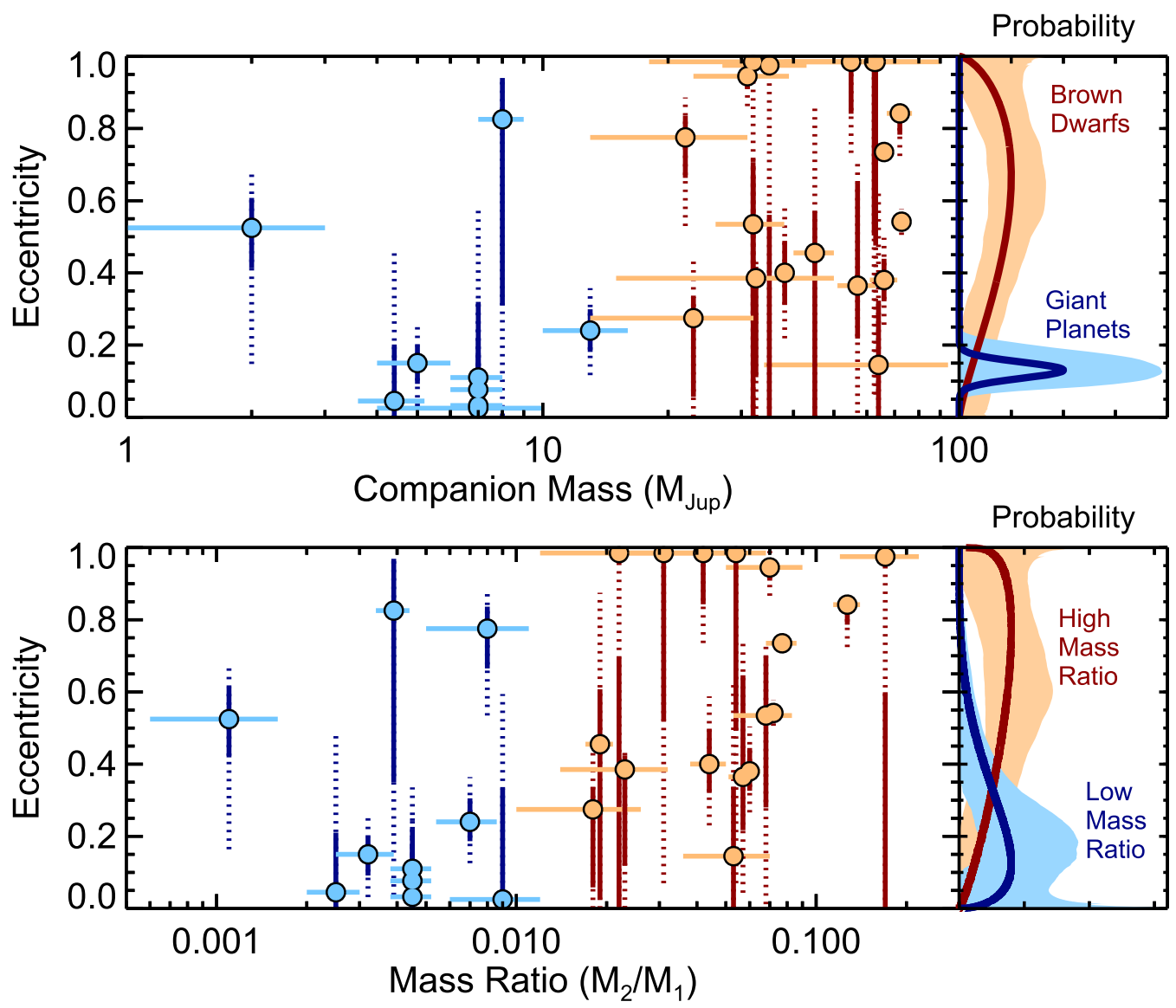

Figure 21. Individual eccentricity distributions for substellar companions between 5 and 100 au as a function of mass (top) and mass ratio (bottom). The inferred population-level eccentricity distributions for subsamples of directly imaged planets and brown dwarf companions are displayed in the panels on the right. Dividing these by a mass threshold of $15 M_{\text {Jup }}$ in the top panel or a mass ratio of 0.01 in the bottom panel does not influence the main conclusion that the eccentricity distribution of directly imaged planets is skewed to low values compared to brown dwarfs. Here blue and orange indicate the subsample divisions we have adopted. The solid and dotted eccentricity uncertainties represent $68 \%$ and $95 \%$ credible intervals, respectively. Shaded regions in the right panels illustrate $2 \sigma$ credible intervals for the eccentricity posteriors.

\subsubsection{A Separation Threshold}

We also examine whether there are differences in the eccentricity distribution as a function of separation, which might be expected if, for example, the inner population of substellar companions predominantly originates within a disk while the outer population largely represents the product of cloud fragmentation. For this experiment, we adopt a threshold of $30 \mathrm{au}$, which is chosen so as to divide the full sample of substellar companions into two approximately equal bins.

Results for the subsample of 14 companions between 5 and 30 au and 13 companions between 30 and 100 au are shown in Figure 22 and are summarized in Table 7 . We find values of $\alpha=1.70_{-0.9}^{+1.2}$ and $\beta=2.8_{-1.2}^{+1.7}$ for the sample at close separations, which corresponds to a broad peak between $e=0.1-0.4$ with significant power from $e=0.0-0.8$. At wide separations, we find $\alpha=0.7_{-0.4}^{+0.6}$ and $\beta=0.9_{-0.3}^{+0.7}$. This corresponds to a roughly flat distribution with somewhat higher power at the bounded endpoints. There is some evidence that the more closely separated population lacks companions at the highest eccentricities, but these two distributions are otherwise broadly similar and are not nearly as distinct as the giant planet and brown dwarf subsamples. The probability that wide companions have higher eccentricities than close companions is $52.3 \%$ ( $2 \sigma$ credible interval: $24.4 \%-81.7 \%$ ).

\subsubsection{Exploring the Influence of HR 8799}

As a system of four giant planets with masses $\gtrsim 5 M_{\text {Jup }}$ and separations between 15 and 70 au, HR 8799 is atypical among directly imaged planetary systems. ${ }^{13}$ Based on the $\approx 1 \%$ occurrence rate of planets at these separations (Bowler 2016; Galicher et al. 2016), the probability of randomly finding four such planets around one star is $\sim 0.01^{4}$, or $10^{-8}$, assuming each planet represents an independent probabilistic event. Only about $10^{3}$ stars have been observed in high-contrast imaging surveys to date, which makes it exceedingly unlikely that these planets are independent of each other. HR 8799 is therefore a special case in which the probability of an additional planet in this system is conditioned on the presence of another one being there. Other planets may of course reside in the apparently single systems at closer separations and lower masses, but HR 8799 appears to be unique in that it has four massive planets detected on wide orbits. The physical underpinning of this is, of

\footnotetext{
${ }^{13}$ HR 8799 has long been the only multi-planet system to be imaged, but it may now be joined by PDS 70 (Haffert et al. 2019), $\beta$ Pic (Lagrange et al. 2019), and LkCa15 (Kraus \& Ireland 2012; Sallum et al. 2015). Note that an additional unknown close-in substellar companion has the potential to bias the astrometry and inferred eccentricities of wider imaged companions (Pearce et al. 2014). Astrometry is calculated with respect to the primary, so a relatively massive inner object can perturb the host star and alter the apparent orbital elements of long-period companions.
} 

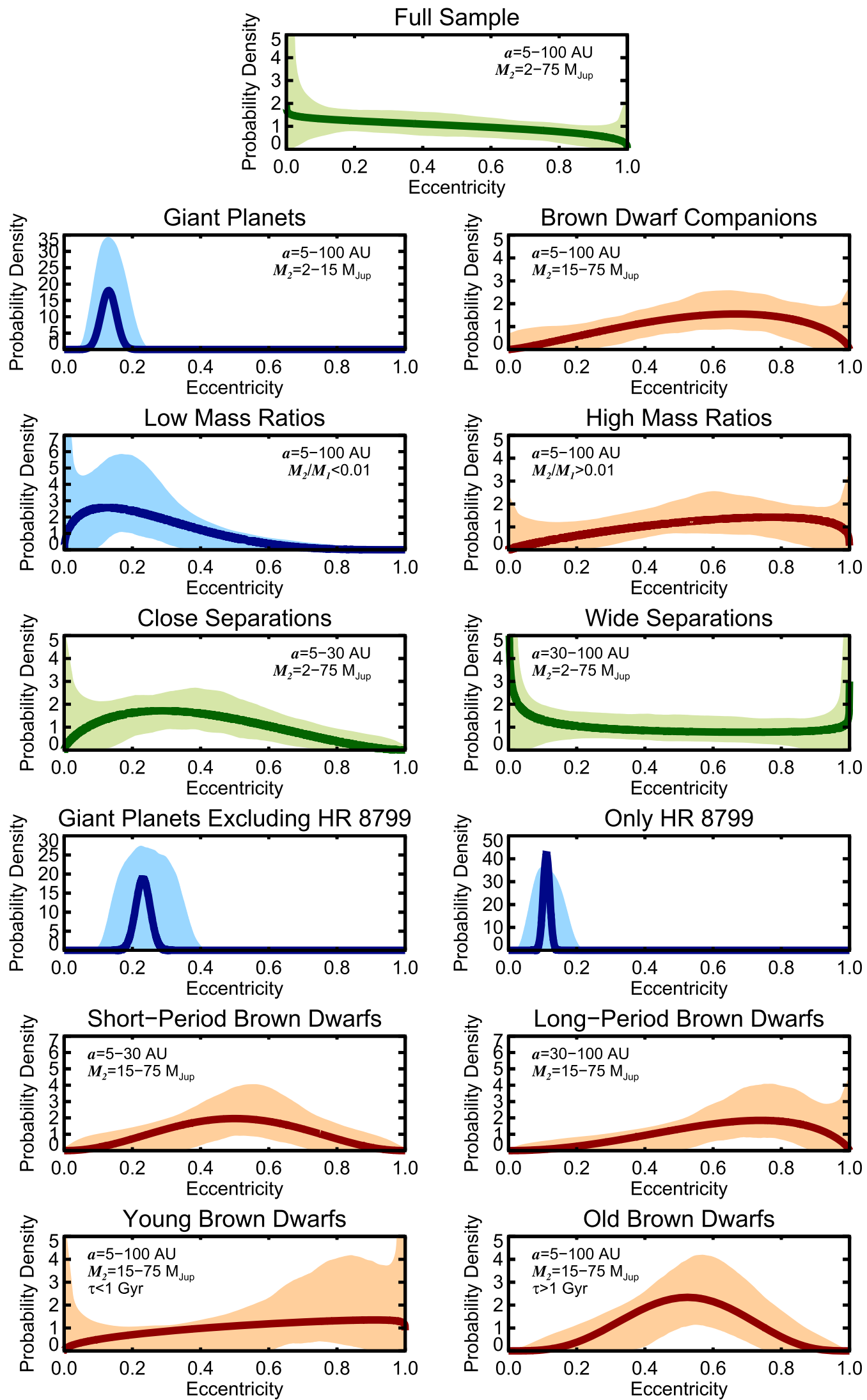

Figure 22. Population-level eccentricity distributions for the full sample of substellar companions between 5 and 100 au (top panel) and various subsamples divided by companion mass, system mass ratio, and orbital separation (second, third, and fourth rows). Results for the planet population excluding HR 8799 and results for HR 8799 alone are displayed in the fifth row. The sixth row shows brown dwarfs at small (5-30 au) and large (30-100 au) orbital separations, and the bottom row displays the recovered eccentricity distributions for young $(<1 \mathrm{Gyr})$ and old $(>1 \mathrm{Gyr})$ brown dwarfs. The thick curve shows the best-fitting Beta distribution for each sample. Shaded regions illustrate the $2 \sigma$ credible intervals for posteriors at each eccentricity. 
course, likely to have been an unusually massive and physically large protoplanetary disk.

The fact that the HR 8799 planets are in an apparently stable, near-circular, approximately coplanar orbital arrangement could bias the results of our eccentricity analysis for the giant planet subsample. Higher eccentricities for any of these planets have the potential to destabilize the system, so we may be imposing an unintentional anthropic bias by including this system in our analysis. For example, if the eccentricities had been high, the planets may not have formed, migrated, or persisted at their present locations and therefore may not have been discovered. It is not clear if the high masses and separations of the HR 8799 planets are what make this system unusual, or perhaps the close dynamical packing of the planets relative to other systems. Nevertheless, because of this unusual status, we carry out two additional tests: one for the giant planet subsample excluding HR 8799 bcde, and one that considers the HR 8799 planets alone to assess constraints on the underlying eccentricity distribution for this planetary system alone.

Results of these experiments are shown in Figure 22, and the hyperparameter values are listed in Table 7 . When we exclude HR 8799 and only consider five planets, we find hyperparameters of $\alpha=90$ ( $1 \sigma$ credible interval: $87-311)$ and $\beta=300$ ( $1 \sigma$ credible interval: 555-1000). Compared to the full sample of giant planets, the corresponding underlying eccentricity distribution broadens and shifts to slightly higher values between $e=0.1-0.4$ with a peak at $\bar{e}=0.23$. The hyperparameters for HR 8799 bcde alone are $\alpha=120$ ( $1 \sigma$ credible interval: 44-127) and $\beta=960$ ( $1 \sigma$ credible interval: 570-1000). This yields a tighter distribution between $e=0.0-0.2$ with a peak at $\bar{e}=0.11$. The full sample of giant planets in Section 4.3.3 reflects intermediate eccentricities between HR 8799 and the rest of the population. Based on these reconstructions, the probability that the HR 8799 planets have lower eccentricities than the other systems is $99.9 \%$ ( $2 \sigma$ credible interval: $78.5 \%-100 \%$ ).

The HR 8799 planets are therefore on somewhat more circular orbits than the other apparently single imaged planets, which is similar to the eccentricity dichotomy between multiplanet and single systems at small separations (Xie et al. 2016). However, as with "single" planets at small separations, we note that single directly imaged systems may harbor additional planets below the detection threshold.

\subsubsection{Short- and Long-period Brown Dwarfs}

Tokovinin \& Kiyaeva (2015) presented the first observational results demonstrating that the eccentricities of wide stellar binaries well outside of the eccentricity-period tidal circularization envelope increase with longer orbital period from a mean value of $e \approx 0.4$ for $10^{2-3}$ days to $e \approx 0.6$ for $10^{5-6}$ days. This may be a reflection of the dissipative interaction of closer binaries with circumstellar disk or envelope material during the formation of the pair (e.g., Bate 2009). If brown dwarf companions have a shared origin with stellar companions, then they may have a similar period dependence on the mean eccentricity.

To test this, we divide our brown dwarf sample into a subsample of nine short-period companions between 5 and $30 \mathrm{au}$ and nine long-period companions spanning 30-100 au. Results are presented in Figure 22 and Table 7. We find values of $\left\{\alpha=3.25_{-2.1}^{+4.8}, \beta=3.25_{-1.9}^{+4.0}\right\}$ for the short-period subsample, which corresponds to an approximately normally distributed function with a peak eccentricity at $\bar{e}=0.50$ and a broad width from $e \approx 0.1-0.9$. The long-period subsample yields $\left\{\alpha=3.1_{-2.0}^{+4.6}, \beta=1.75_{-1.1}^{+1.6}\right\}$, which gives a strongly leftskewed distribution with a peak at $\bar{e}=0.74$ and a broad range from $e \approx 0.2-1.0$. The probability that long-period brown dwarfs have higher eccentricities than their short-period counterparts is $70.0 \%$ ( $2 \sigma$ credible interval: $43.5 \%-98.4 \%$ ). This increasing mean eccentricity with orbital period appears to follow the same trend as is observed with stellar binaries and supports a common formation channel ${ }^{14}$

\subsubsection{Young and Old Brown Dwarfs}

The architectures of planetary systems can evolve through three-body Kozai-Lidov oscillations or dynamical scattering events, both of which can influence the observed eccentricities of giant planets. Indeed, several of the systems in our sample have wide stellar companions (HD 1160, 51 Eri, and HD 4113) that could perturb the eccentricities of inner objects over long timescales. Our planet sample comprises too few objects to explore age effects for this population, but our brown dwarf sample includes systems spanning a wide age range from ₹23 Myr (for PZ Tel) to 6-10 Gyr (for Gl 758).

Here we explore whether there is evidence for a difference in the eccentricity distributions of young and old brown dwarfs. To produce similarly sized bins, we adopt an age of $1 \mathrm{Gyr}$ as a threshold for each subsample. Altogether, there are 11 young brown dwarf companions $(<1 \mathrm{Gyr})$ and 7 old systems $(>1 \mathrm{Gyr})$. Results from our hierarchical Bayesian modeling are shown in Figure 22 and Table 7 . For our young subsample we find hyperparameter values of $\alpha=1.50_{-1.29}^{+4.11}$ and $\beta=1.05_{-0.64}^{+1.24}$, which correspond to a broad eccentricity distribution with a general preference for high values. The older sample yields $\alpha=4.70_{-2.33}^{+4.90}$ and $\beta=4.35_{-2.02}^{+3.52}$, which is approximately Gaussian-shaped and centered at $\bar{e}=0.52$, with the highest power between $e \approx 0.2-0.8$. The uncertainties are relatively large on these inferred shapes, which is reflected in the probability that young brown dwarfs have higher eccentricities than old brown dwarfs: $59.7 \%$ with a $2 \sigma$ credible interval of $35.2 \%-99.7 \%$. We therefore do not find significant evidence for distinct distributions among brown dwarf companions when they are subdivided by system age.

\subsection{Discovery Bias}

A direct imaging survey will preferentially find close-in companions with higher eccentricities compared to companions that have the same semimajor axes but are on circular orbits (e.g., Dupuy \& Liu 2011; Kane 2013). This is because more eccentric orbits will reach wider apastron distances and therefore companions will spend more time at large separations compared to those with lower eccentricities. This preference produces a bias that can skew the apparent eccentricity distribution of discoveries toward high eccentricities. Discovery bias is strongest when the semimajor axis is much smaller than the IWA (at the same contrast as the companion), and it

\footnotetext{
${ }^{14}$ Note that brown dwarfs at wider separations have longer orbital periods and are thus more susceptible to the orbit fits being influenced by systematic errors in the astrometry. However, they are also easier to discover earlier than shortperiod brown dwarfs, so more time has generally elapsed to monitor their orbits, which partly makes up for this potential bias (albeit only slightly). Altogether, we expect significantly better constraints and more reliable fits for the shorter period companions. Ultimately, larger samples and continued astrometric monitoring of known systems are needed to confirm this trend of increasing eccentricity with separation.
} 
asymptotically disappears for companions with semimajor axes well beyond the IWA. This metric-IWA $/ a$-is therefore a useful tool to assess whether a given sample is skewed to higher eccentricities from discovery bias.

Our sample of substellar companions draws from an assortment of AO imaging surveys carried out over the past two decades that had a wide range of sensitivities, inner working angles, and resulting contrast curves. Moreover, targets in our sample span a broad range of distances from $5.8 \mathrm{pc}$ (Gl 229) to $126 \mathrm{pc}$ (HD 1160); for a given contrast curve, more distant systems may be more heavily biased in favor of higher eccentricities for the same reasons shorter period companions are preferentially selected against.

If our sample is strongly influenced by discovery bias, then we would expect the eccentricities of our targets to exhibit several trends that are characteristic of this preferential selection. Below we discuss five such correlations in detail to assess the potential impact of this bias on our results.

1. The IWA at the time of discovery should be comparable to or larger than the semimajor axes of companions in our sample. High values of IWA/ $a$ would suggest that a strong bias is likely present, while low values below unity would indicate minimal bias.

The IWA here should be at the same contrast as the companion and must be determined at the time the companion was first imaged. We therefore revisited the original discovery papers for each system in our sample and visually estimated the IWA from the discovery images. These are then converted into physical units using the distance to the system and divided by the maximum a posteriori of the semimajor axis posterior from Table 3 (or values from the literature for systems that we did not refit in this study).

The distribution of IWA/ $a$ for our sample is shown in Figure 23, and individual values are listed in Table 4. Thirteen systems from our full sample have IWA/ $a<0.5,12$ systems have $0.5 \leqslant$ IWA $/ a<1.0$, and 2 systems (HD 1160 B and HD 49197 B) have IWA/ $a \geqslant 1.0$. Dupuy \& Liu (2011) simulated the impact of discovery bias as a function of both IWA $/ a$ and eccentricity (see their Figure 1) and found that there is minimal impact on the relative fraction of detected systems across all eccentricities for values of IWA/ $a \lesssim 0.5$. For IWA $/ a$ values between $\approx 0.5-1$, there is a modest preferential suppression of low-eccentricity orbits. For values above IWA $/ a \approx 1.0$, this suppression becomes severe and only the highest eccentricities are detectable. We expect that about half of our sample suffers from minimal discovery bias and about half is moderately influenced by this effect.

2. There should be a correlation between IWA $/ a$ and eccentricity. Objects with semimajor axes well outside the IWA should be minimally biased, whereas those at or inside the IWA should preferentially be more eccentric.

The lower panel of Figure 23 shows the eccentricity of each system as a function of IWA $/ a$. There is a broad range of eccentricities for IWA $/ a$ values between 0.0-1.0. The two systems with the highest values of IWA/a (HD 1160 B and HD 49197 B) have poorly constrained eccentricities, but these distributions favor higher values of $e$. This suggests that these two
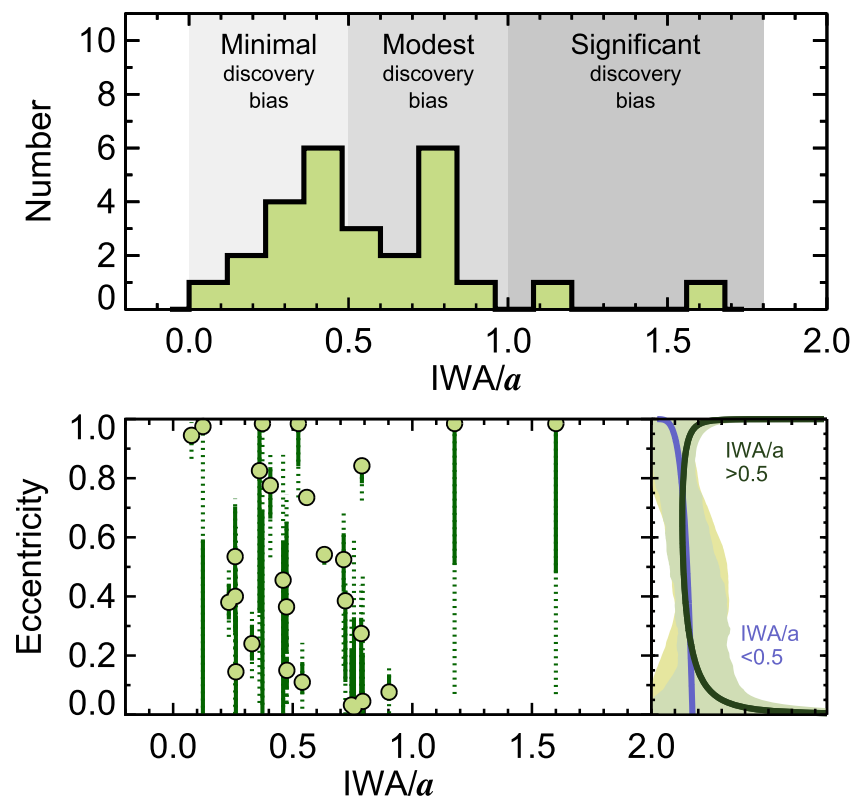

Figure 23. Top: distribution of IWA/ $a$ values for brown dwarf companions and giant planets in our sample at the time of their discovery. Discovery bias is expected to preferentially suppress low eccentricities for high values of IWA $/ a$ $(\gtrsim 1)$. Values of IWA $/ a$ between 0.5 and 1.0 are modestly affected by discovery bias, and values below 0.5 are minimally influenced (Dupuy \& Liu 2011). Bottom: eccentricity as a function of IWA/ $a$ for targets in our sample. Discovery bias is expected to imprint higher eccentricities for higher values of IWA $/ a$. There is some evidence for this for the two systems with IWA/ $a$ values above 1.0, whose eccentricities are poorly constrained but favor high values, but the inferred eccentricity distribution for IWA $/ a<0.5$ and IWA $/ a>0.5$ does not reveal any noticeable trend (bottom right). This suggests that the observed population-level eccentricity distribution is not substantially shaped by discovery bias.

companions are probably influenced by this bias, but there is no obvious trend for the rest of the sample. This is reflected in the reconstructed eccentricity distributions for systems with IWA $/ a<0.5$ and IWA $/ a>0.5$, which both have approximately flat shapes spanning $e=0-1$.

3. More distant systems should have higher eccentricities, on average, as IWA $/ a$ becomes larger and this bias becomes stronger. A positive correlation between the population-level eccentricity distribution and the distance to the system would be another indicator that discovery bias may be important.

To test whether our results for brown dwarf companions in Section 4 may be skewed to high values because of discovery bias, we reassess the populationlevel eccentricities for nearby $(<40 \mathrm{pc})$ and distant $(>40 \mathrm{pc})$ subsamples. Results from this exercise are shown in Figure 24 and Table 7. The recovered eccentricity distribution for nearby brown dwarfs peaks at high values $(\bar{e} \approx 0.7)$. The best-fitting distribution for distant systems is flat across all eccentricities, with an overdensity of posterior values at modest eccentricities near $e=0.5$. This apparent tendency for closer systems to have higher eccentricities is opposite to the trend we would expect if discovery bias played a strong role in shaping the population-level eccentricity distribution. With only eight objects in the nearby sample and nine in the distant sample, we expect that the slight differences we observe are caused by small number statistics. 


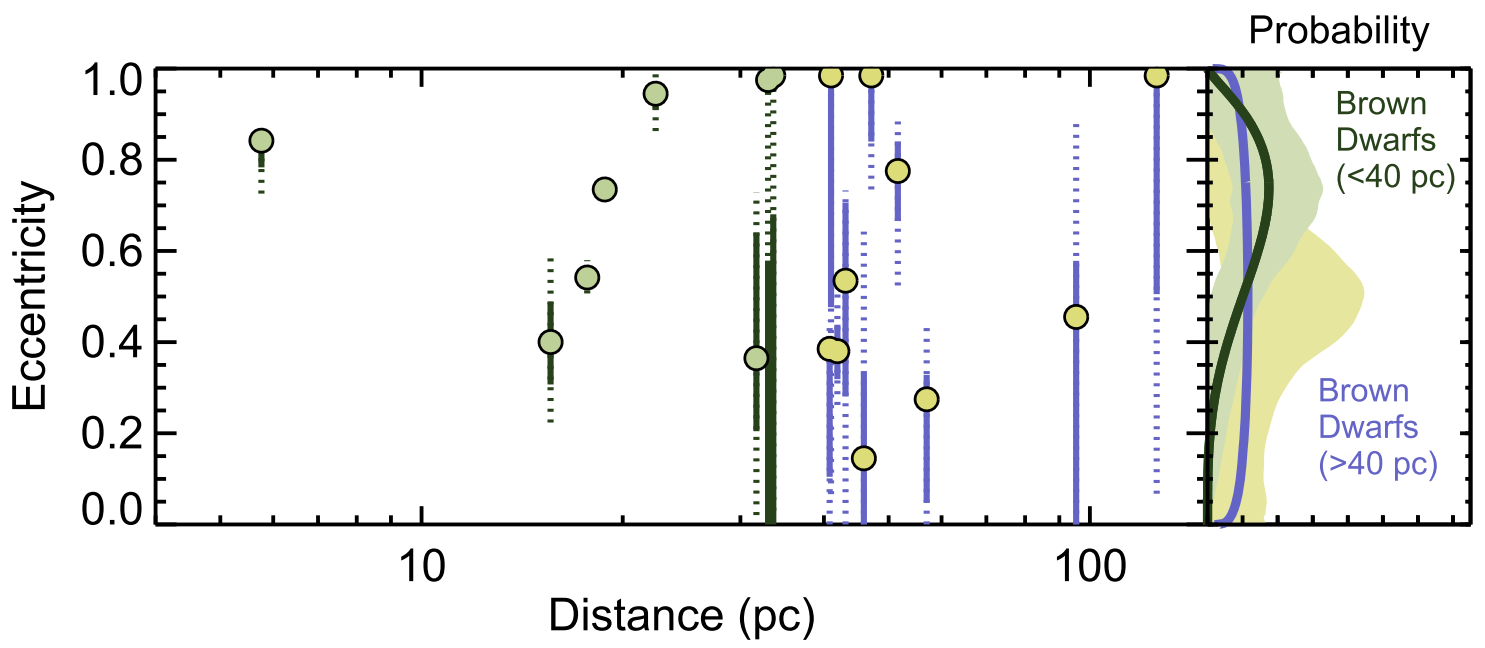

Figure 24. Eccentricities of brown dwarf companions as a function of distance. For a given contrast curve, discovery bias is expected to more strongly select for higher eccentricities at larger distances. We find the opposite trend for the recovered population-level eccentricity distributions for the nearby $(<40 \mathrm{pc})$ and distant ( $>40 \mathrm{pc}$ ) subsamples (right panel): the nearby sample is skewed to higher eccentricities, while the best-fitting Beta distribution is flat for the more distant systems. Shaded regions represent $2 \sigma$ credible intervals for each eccentricity. Note that the peak in the uncertainty profile for distant brown dwarfs at $e \approx 0.5$ results from a pile-up of tighter posteriors in this region, even though the best fit is approximately uniform.

4. Lower mass companions with higher contrasts relative to their host stars should be more strongly biased toward high eccentricities compared to higher mass (lower contrast) companions. This is because contrast curves are not constant, but typically curve to larger angular separations at higher contrasts. That is, the effective IWA increases at lower companion masses.

In Section 4 we showed that the inferred eccentricity distribution of giant planets in our sample is more circular than the distribution for brown dwarf companions. This is opposite of what we would expect if discovery bias played a significant role in shaping these observed distributions.

5. Companions with shorter orbital periods should be more eccentric than their counterparts at wider separations.

In Section 4 we derived the underlying eccentricity distributions for substellar companions at separations between 5-30 au and 30-100 au. The close-separation subsample exhibited a broad peak at $\bar{e} \approx 0.3$ with a paucity of power at the highest eccentricities. The wideseparation subsample was essentially flat across all eccentricities. Once again, these trends are opposite of what we would expect if discovery bias played a dominant role in shaping these distributions.

Altogether, these series of tests argue against discovery bias playing a major role in shaping the population-level eccentricity distribution of substellar companions in our sample. It is possible (and likely) that some bias is present in this sample, but the eccentricity distributions we inferred in Section 4 appear to predominantly reflect the intrinsic properties of substellar companions. We conclude that our primary finding that brown dwarf companions are more eccentric than giant planets is robust against discovery bias.

\section{Discussion}

One of the overarching motivations for large high-contrast imaging surveys is to determine the dominant pathway(s) through which giant planets and brown dwarfs form and subsequently evolve. This is an especially challenging task at wide separations where occurrence rates are low and there are orders of magnitude fewer discoveries compared to shortperiod planetary systems. There have been many proposed mechanisms to form and preserve substellar companions at separations of tens to hundreds of au: pebble accretion (Johansen \& Lambrechts 2017), disk fragmentation (e.g., Dodson-Robinson et al. 2009; Kratter et al. 2010), outward scattering processes (e.g., Boss 2006; Veras et al. 2009; Bromley \& Kenyon 2014), disrupted inward migration (e.g., Nayakshin 2017), direct cloud fragmentation (Bate et al. 2002), outward scattering plus stellar flybys to generate "Oort planets" (Bailey \& Fabrycky 2019), and dynamical recapture of freefloating planets (Perets \& Kouwenhoven 2012).

Several observational signatures of these formation pathways are expected to be imprinted on the orbital properties and atmospheric compositions of planets and brown dwarfs at wide separations, including their orbital architectures (e.g., Boley 2009), abundance ratios and metallicities (e.g., Fortney et al. 2008; Oberg et al. 2011; Spiegel \& Burrows 2012), luminosities and entropy (e.g., Marley et al. 2007; Marleau \& Cumming 2014), and companion mass function (Reggiani et al. 2016). However, because the occurrence rate of giant planets and brown dwarf companions is so low-about $1 \%$ for the former and about $2 \%-$ $4 \%$ for the latter (Bowler \& Nielsen 2018) - most individual surveys typically find only a small handful of substellar companions (e.g., Metchev \& Hillenbrand 2009; Bowler et al. 2015a; Chauvin et al. 2015; Galicher et al. 2016; Stone et al. 2018; Nielsen et al. 2019). Alone, these are not sufficient for robust assessments of formation scenarios, but as an ensemble, they provide clues about the physical processes, timescales, efficiency, and evolution of planet formation at wide separations.

In this work we have aimed to test whether brown dwarfs and giant planets form in the same fashion based on orbital expectations from two scenarios that most closely resemble the planet and star formation processes: formation within a disk, and fragmenting collapse of a molecular cloud core. Our analysis of the population-level eccentricity distributions for substellar companions between 5 and 100 au shows a clear 
difference between the orbital properties of planetary-mass companions and those in the brown dwarf mass regime. The low-mass companions $\left(<15 M_{\text {Jup }}\right)$ and low mass ratio systems $\left(M_{2} / M_{1}<0.01\right)$ preferentially have lower eccentricities, similar to the population of warm Jupiters at small separations. The brown dwarf companions (15-75 $\left.M_{\text {Jup }}\right)$ and higher mass ratio systems $\left(M_{2} / M_{1}=0.01-0.2\right)$ exhibit higher eccentricities, and there is evidence for a period dependence on the eccentricity distribution analogous to results from Tokovinin \& Kiyaeva (2015) for stellar binaries. This is especially pronounced when compared with the roughly flat eccentricity distribution for brown dwarfs at small separations found by $\mathrm{Ma}$ \& Ge (2014). Moreover, this difference in underlying eccentricity distributions based on mass and mass ratio are the clearest of any other subdivision we tested. The simplest explanation is that these populations predominantly form in distinct manners: the planetary-mass companions originate in disks, while brown dwarf companions represent the low mass ratio end of binary star formation. This complements the conclusions reached by Chabrier et al. (2014) that free-floating brown dwarfs most likely form as the low-mass end of the star formation process based on a range of kinematic, environmental, and statistical characteristics in common with stars.

There are more subtle details about the giant planet eccentricity distribution that may provide further insight into the formation and dynamical histories of these objects. It is interesting to note that like warm Jupiters, imaged planets and binaries with a low mass ratio have an extended, dynamically hot tail to modest eccentricities. 51 Eri $b$ is a notable example: it is the directly imaged planet with the lowest mass and has an unusually high eccentricity $\left(e=0.50_{-0.08}^{+0.11}\right.$ ) compared to the rest of the imaged planets in our sample with decent orbital constraints. At small separations the shape of the warm Jupiter eccentricity distribution is generally interpreted as evidence for gravitational planet scattering and secular three-body interactions (e.g., Ford \& Rasio 2008; Jurić \& Tremaine 2008; Petrovich \& Tremaine 2016). The same may be true for imaged planets: they may comprise a mix of low-eccentricity systems and companions that have been dynamically perturbed to modest eccentricities.

Another feature of close-in planets is that they exhibit an eccentricity dichotomy in which multi-planet systems have lower eccentricities, on average, compared to systems with a single (known) planet (Wright et al. 2009; Limbach \& Turner 2015; Xie et al. 2016; Van Eylen et al. 2019). ${ }^{15} \mathrm{We}$ find the first evidence of a similar phenomenon at wide separations: the eccentricity distribution of the four HR 8799 planets peaks at $\bar{e}=0.11$, whereas the distribution for single imaged planets shifts to $\bar{e}=0.23$ and broadens when HR 8799 is excluded. If confirmed with additional systems such as PDS $70 \mathrm{bc}$ and $\beta$ Pic bc in the future (Haffert et al. 2019; Lagrange et al. 2019), this would suggest that long-period multi-planet systems have probably not experienced strong scattering in their past, whereas single imaged systems were probably excited at some point, perhaps by another planet. Together, this could indicate that most systems with jovian planets have, or once harbored, multiple long-period planets.

\footnotetext{
${ }^{15}$ Note that Bryan et al. (2016b) found that that this trend tends to reverse when long-term accelerations are considered, which are sensitive to planetary companions out to about $10 \mathrm{au}$. When this is taken into account, two-planet systems tend to have higher eccentricities than single-planet systems, perhaps a result of dynamical interactions.
}

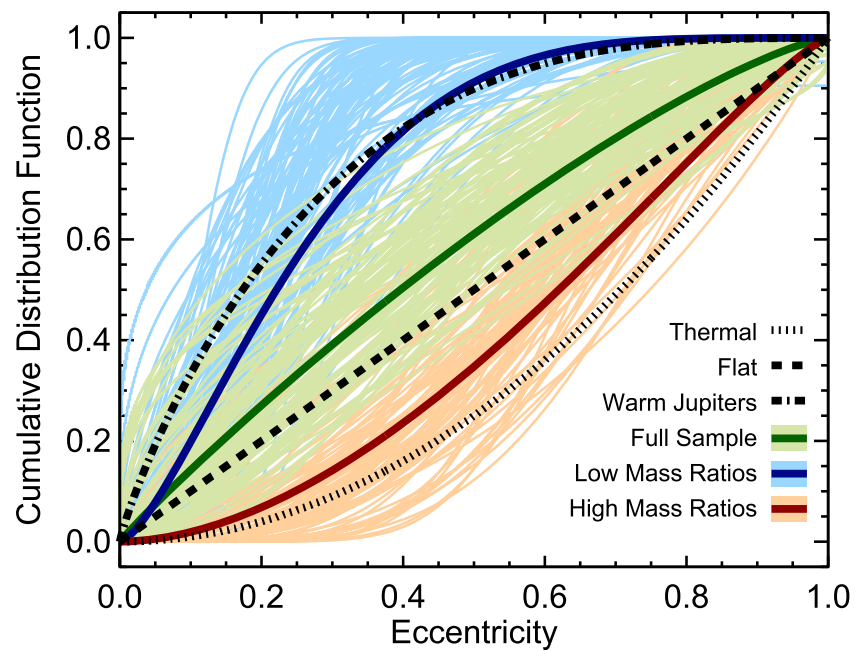

Figure 25. Cumulative distribution functions for our full sample of substellar companions (green), the subset of low mass ratio systems (blue), and the subset of high mass ratio systems (red). The low mass ratio subsample resembles the warm Jupiter eccentricity distribution (dot-dashed curve) from Kipping (2013), while the high mass ratio systems lie between a flat distribution $(f(e)=$ const.; dashed curve) and thermal distribution $(f(e)=2 e$; dotted curve). Non-tidally circularized stellar binaries (not plotted) reside between the exoplanet and flat CDFs (Duchêne \& Kraus 2013). One hundred randomly drawn CDFs are displayed for each subsample.

In Figure 25 we compare eccentricity CDFs for our low mass ratio subsample, high mass ratio subsample, and full sample of substellar companions to the eccentricity distribution of warm Jupiters from Kipping (2013), a flat distribution, and a thermal distribution (Ambartsumian 1937). The best-fitting distribution of low mass ratio companions between 5 and $100 \mathrm{au}$ is remarkably similar to that of close-in giant planets, which suggests that this functional form does not appear to strongly vary from $\approx 0.1$ au out to $100 \mathrm{au}$. Note, however, that these populations are quite different: the warm Jupiters have lower masses $\left(\approx 0.1-10 M_{\text {Jup }}\right.$ with a bottom-heavy functional form) and older ages (typically several Gyr), whereas the planets in our sample have high masses $\left(2-15 M_{\text {Jup }}\right)$ and young ages ( $\lesssim 50 \mathrm{Myr}$ ). Nevertheless, the resemblance is noteworthy and may reflect something fundamental about the formation and early dynamical interactions of giant planets.

The eccentricity distribution of solar-type visual and spectroscopic binary stars outside the tidal circularization radius (12 days $\lesssim P \lesssim 10^{6}$ days; $a \approx 0.1-200 \mathrm{au}$ ) is approximately flat, perhaps with a slight peak at modest eccentricities, and falls between the CDFs for warm Jupiters and flat distributions (Raghavan et al. 2010; Duchêne \& Kraus 2013). Tokovinin \& Kiyaeva (2015) showed that the eccentricities of solar-type stellar binaries progressively increase with increasing orbital period from $10^{2}$ days to $10^{6}$ days $(a \approx 0.4$ au to $200 \mathrm{au})$ toward the thermal distribution curve $(f(e) \sim 2 e)$. We found that the eccentricity distribution for all substellar companions is approximately uniform, which is consistent with (but slightly flatter than) that of close-in stellar binaries. The brown dwarf and high mass ratio eccentricity CDFs fall below that of the uniform distribution and more closely follow a thermal distribution. Most of the targets in our analysis are young $(\lesssim 200 \mathrm{Myr})$, so these results largely probe the dynamical conditions of these systems soon after formation and generally before several gigayears of potential evolution. As suggested by Geller et al. (2019), these distributions are 
therefore likely to reflect the intrinsic properties of brown dwarf companions rather than long-term dynamical processing.

This work joins other recent studies that have found evidence for population-level differences between brown dwarfs and imaged planets on wide orbits. Nielsen et al. (2019) presented statistical results from the first 300 stars in the GPIES survey. They found a positive correlation between the frequency of giant planets and stellar host mass, a bottomheavy planet mass distribution, and generally shorter orbital periods for the planetary companions. The brown dwarfs in their sample exhibited the opposite trends. Nielsen et al. interpret these observations as evidence in favor of core/pebble accretion for giant planets and gravitational fragmentation of disks for brown dwarfs. Wagner et al. (2019) analyzed the underlying relative mass distribution of substellar companions using survival analysis and found a bottom-heavy mass function that resembles that of close-in planets. This similarity suggests formation from core accretion for planets below $\approx 10-20 M_{\text {Jup }}$. Our results that lower mass companions have more circular orbits bolsters these conclusions. A positive correlation with stellar mass, a bottom-heavy mass function, and low-eccentricity orbits all point to bottom-up formation in disks.

Our relatively modest sample of 27 giant planets and brown dwarf companions has limited our assessment to broad trends in the shape of the eccentricity distribution function. This has left open a number of questions related to the formation and evolution of long-period substellar companions:

1. Where is the dynamical distinction between giant planets and brown dwarfs? We have adopted thresholds of $15 M_{\text {Jup }}$ and $M_{2} / M_{1}=0.01$ for this study, but we do not have the statistical leverage to test the mass or mass ratio at which this difference arises, or whether it is a smooth transition toward more circular orbits at lower masses.

2. Do the population-level eccentricities of substellar companions evolve over time, or are they established at young ages? Most direct imaging surveys have preferentially focused on young stars. This bias is reflected in the young ages of most targets in our sample. Substellar companions spanning a wide range of ages (1 Myr to $10 \mathrm{Gyr}$ ) will provide a probe of the dynamical evolution of these systems over time.

3. Does the observed period-eccentricity trend continue at wider separations? The current astrometric precision from AO observations at large telescopes is $\approx 1$ mas. Orbital motion of wider companions out to several hundred au can readily be detected with this precision after a few years of monitoring. An expanded analysis to longer periods will establish whether the eccentricity distribution of brown dwarfs continues to peak at higher values at wider separations.

4. Is the eccentricity dichotomy at wide separations real? Our analysis of the HR 8799 multi-planet system and the rest of the imaged planet population within $100 \mathrm{au}$ suggests that single systems appear to be dynamically hot. More discoveries are needed to establish whether this is a universal property of single versus multiple planets on wide orbits, or if it reflects peculiarities in the orbits of HR 8799 bcde. In addition, higher contrast observations at smaller angular separations will establish whether these apparently single systems have closer-in companions.
5. How do these eccentricity distributions depend on stellar multiplicity, mass, and metallicity? We restricted our study to mostly include single stars to avoid the dynamical influence of stellar binaries at close and wide separations. We also largely ignored the effect of host mass or composition in this work. All of these parameters influence the properties of planets at close separations, so it is natural to consider assessing them at wide separations. Broader samples that include a diversity of host star properties are needed to explore how these characteristics influence long-period substellar companions.

These questions can be addressed through continued orbit monitoring of known systems and larger samples of imaged planets and brown dwarf companions. With few exceptions, these targets have largely been found in blind AO imaging surveys. Transitioning toward "informed targets" with dynamical evidence of a long-period low-mass companion is a promising approach to improve the efficiency of these discoveries. In particular, the final Gaia data release is expected to deliver tens of thousands of giant planets within $10 \mathrm{au}$ (e.g., Perryman et al. 2014). Astrometric accelerations from Gaia will eventually point the way to wider substellar companions.

In the near term, the Hipparcos-Gaia Catalog of Accelerations-a cross-calibrated catalog of astrometry from Hipparcos and Gaia developed by Brandt (2018)_offers an especially promising pathway to identify long-period substellar companions over the next few years (see also Kervella et al. 2019). This can be facilitated with the complementary nature of extreme AO systems on large telescopes (Jovanovic et al. 2015; Macintosh et al. 2014; Beuzit et al. 2019) and autonomous instruments such as Robo-AO (Baranec et al. 2014) on smaller telescopes to systematically and efficiently survey these new candidates. Furthermore, in the future, the next generation of $30 \mathrm{~m}$ class telescopes will substantially increase this landscape by probing lower planet masses, closer separations, and older ages.

\section{Summary and Conclusions}

In this study we have carried out the first population-level analysis of the eccentricities of directly imaged planets and brown dwarf companions. We first presented new AO observations of 13 substellar companions from Keck/NIRC2 and Subaru/HiCIAO along with updated orbit fits to these systems using orbitize!, which is optimized for systems with small fractional orbit coverage (Sections 2 and 3). We identified a sample of 27 companions between 5-100 au with masses under $75 M_{\text {Jup }}$ that are undergoing orbital motion; for nine systems we assembled astrometry from the literature and uniformly refit their orbits (Section 4.2 and Table 3).

Assuming a Beta distribution as a flexible model for the underlying population-level eccentricity distribution, we determined the overall behavior of substellar orbital eccentricities within the framework of hierarchical Bayesian inference. Following the importance sampling approach from Hogg et al. (2010), individual posterior eccentricities from our orbit fits are used to approximate the likelihood function, then hyperparameter posteriors are sampled with MCMC. This procedure was carried out for the full sample of substellar companions as well as various subdivisions by companion mass, system mass ratio, separation, system architecture, and 
age. Finally, we assessed the potential role of discovery bias in shaping our results (Section 4.4). Below is a summary of our main conclusions.

1. The primary result from this study is that the underlying eccentricity distributions for directly imaged planets (2-15 $\left.M_{\text {Jup }}\right)$ and brown dwarf companions (15-75 $\left.M_{\text {Jup }}\right)$ between 5 and 100 au are significantly different. Giant planets have low orbital eccentricities with a peak at $\bar{e}=0.13$, while brown dwarfs exhibit a broad distribution with a preference for higher eccentricities and a peak in the distribution function between $\bar{e}=0.6-0.9$. The corresponding Beta distributions have shape parameters of $\{\alpha=30, \beta=200\}$ for imaged planets and $\{\alpha=2.30, \beta=1.65\}$ for brown dwarf companions. The eccentricity trends hold whether subdivided by mass $\left(15 M_{\text {Jup }}\right)$ or mass ratio $\left(M_{2} / M_{1}=0.01\right)$. We interpret this as evidence for formation within a disk for the imaged planets and from cloud fragmentation for brown dwarfs. These differences in dynamical properties based on mass or mass ratio are the clearest of any other subdivision we tested.

2. The underlying eccentricity distribution function for the low mass ratio subsample bears a close resemblance to the distribution of warm Jupiters outside of the tidal circularization radius from radial velocity surveys. Lowmass ratio companions do not reside on circular orbits but have a dynamically hot tail out to modest eccentricities of $\approx 0.6$. This may indicate that dynamical heating from scattering events plays a role in shaping the orbital properties of directly imaged planets.

3. We find evidence that the eccentricity dichotomy between single and multi-planet systems also exists at wide separations. The HR 8799 planets have more circular orbits $(\bar{e}=0.11)$ with a narrower range of eccentricities compared to systems with single long-period giant planets $(\bar{e}=0.23)$.

4. There is evidence that brown dwarfs have higher eccentricities at longer orbital periods. Brown dwarf companions on closer orbits (5-30 au) have a "softer" eccentricity distribution with a peak at $\bar{e}=0.50$ compared to those at wider separations (30-100 au), which peak at $\bar{e}=0.74$. This is similar to results for wide $(>50 \mathrm{au})$ stellar binaries from Tokovinin \& Kiyaeva (2015), suggesting a shared formation channel for these populations.

5. The full substellar population from 2 to $75 M_{\text {Jup }}$ and 5-100 au is approximately flat across all eccentricities. This represents a balance between the more circular giant planets and more eccentric brown dwarfs. The best-fitting Beta shape parameters are $\{\alpha=0.95 ; \beta=1.30\}$.

6. Our sample appears to be robust against discovery bias, which tends to preferentially select high eccentricities for systems with semimajor axes comparable to or below the IWA. The distribution of IWA/ $a$ for our sample at the time of discovery is largely below unity, and we find no correlations with eccentricity, distance to the system, orbital distance, or companion mass in the way that would be expected if discovery bias played a major role in shaping the inferred population-level eccentricity distributions.
7. The linear evolution of separation and position angle over time has been uniformly measured for 21 systems with substellar companions using all available astrometry to date. These fits may be helpful for future orbit-monitoring purposes and spectroscopic characterization, for example, with the James Webb Space Telescope or new fiber injection units. Linear relations are presented in Table 2 for convenience.

We thank the anonymous referee for constructive suggestions that improved the quality of this manuscript; Jason Wang, Erik Petigura, Michael Liu, and Quang Tran for helpful feedback on this study; and Eugene Chiang for discussions about long-period planet formation. The eccentricity posteriors for Gl 229 B and the HR 8799 planets were shared by Tim Brandt and Jason Wang. Brian Mason provided astrometry in the WDS catalog for Ross 458.

B.P.B. acknowledges support from the National Science Foundation grant AST-1909209. S.B. is supported by the NSF Graduate Research Fellowship, grant No. DGE 1745303. This work was supported by a NASA Keck PI Data Award, administered by the NASA Exoplanet Science Institute. Data presented herein were obtained at the W. M. Keck Observatory from telescope time allocated to the National Aeronautics and Space Administration through the agency's scientific partnership with the California Institute of Technology and the University of California. The Observatory was made possible by the generous financial support of the W. M. Keck Foundation.

This publication makes use of data products from the Two Micron All Sky Survey, which is a joint project of the University of Massachusetts and the Infrared Processing and Analysis Center/California Institute of Technology, funded by the National Aeronautics and Space Administration and the National Science Foundation This work has made use of data from the European Space Agency (ESA) mission Gaia (https://www. cosmos.esa.int/gaia), processed by the Gaia Data Processing and Analysis Consortium (DPAC, https://www.cosmos.esa.int/ web/gaia/dpac/consortium). Funding for the DPAC has been provided by national institutions, in particular the institutions participating in the Gaia Multilateral Agreement. NASA's Astrophysics Data System Bibliographic Services together with the VizieR catalog access tool and SIMBAD database operated at CDS, Strasbourg, France, were invaluable resources for this work. The authors wish to recognize and acknowledge the very significant cultural role and reverence that the summit of Maunakea has always had within the indigenous Hawaiian community. We are most fortunate to have the opportunity to conduct observations from this mountain.

Facilities: Keck:II (NIRC2), Subaru (HiCIAO).

\section{Appendix A Literature Astrometry}

Published astrometry used in this analysis (Table 8). When previously published observations were re-reduced and presented in subsequent studies, we generally adopted the more recent measurements for this work. 
Table 8

Literature Astrometry

\begin{tabular}{|c|c|c|c|c|}
\hline Name & $\begin{array}{l}\text { Epoch } \\
\text { (UT) }\end{array}$ & $\begin{array}{l}\text { Separation } \\
\text { (mas) }\end{array}$ & $\begin{array}{l}\text { P.A. } \\
\text { (deg) }\end{array}$ & Reference \\
\hline HD 984 B & 2012.545 & $190 \pm 20$ & $109 \pm 3$ & Meshkat et al. (2015) \\
\hline HD $984 \mathrm{~B}$ & 2012.550 & $208 \pm 23$ & $109 \pm 3$ & Meshkat et al. (2015) \\
\hline HD 984 B & 2014.687 & $201.6 \pm 0.4$ & $92.2 \pm 0.5$ & Meshkat et al. (2015) \\
\hline HD 984 B & 2015.657 & $216.3 \pm 1.0$ & $83.3 \pm 0.3$ & Johnson-Groh et al. (2017) \\
\hline HD 984 B & 2015.657 & $217.9 \pm 0.7$ & $83.6 \pm 0.2$ & Johnson-Groh et al. (2017) \\
\hline HD 1160 B & 2002.570 & $770 \pm 30$ & $246.2 \pm 1.0$ & Nielsen et al. (2012) \\
\hline HD 1160 B & 2003.838 & $770 \pm 30$ & $245.6 \pm 1.0$ & Nielsen et al. (2012) \\
\hline HD $1160 \mathrm{~B}$ & 2005.975 & $760 \pm 30$ & $244.7 \pm 1.0$ & Nielsen et al. (2012) \\
\hline HD $1160 \mathrm{~B}$ & 2008.503 & $800 \pm 60$ & $245 \pm 2$ & Nielsen et al. (2012) \\
\hline HD $1160 \mathrm{~B}$ & 2010.710 & $770 \pm 60$ & $243 \pm 2$ & Nielsen et al. (2012) \\
\hline HD $1160 \mathrm{~B}$ & 2010.830 & $780 \pm 30$ & $244.3 \pm 0.2$ & Nielsen et al. (2012) \\
\hline HD 1160 B & 2010.890 & $760 \pm 30$ & $244.5 \pm 0.2$ & Nielsen et al. (2012) \\
\hline HD $1160 \mathrm{~B}$ & 2010.904 & $770 \pm 20$ & $244.9 \pm 0.5$ & Nielsen et al. (2012) \\
\hline HD 1160 B & 2011.523 & $780 \pm 30$ & $244.0 \pm 1.0$ & Nielsen et al. (2012) \\
\hline HD $1160 \mathrm{~B}$ & 2011.669 & $780 \pm 30$ & $244.9 \pm 1.0$ & Nielsen et al. (2012) \\
\hline HD 1160 B & 2011.803 & $770 \pm 30$ & $244.5 \pm 0.2$ & Nielsen et al. (2012) \\
\hline HD $1160 \mathrm{~B}$ & 2011.852 & $780 \pm 30$ & $244.4 \pm 1.0$ & Nielsen et al. (2012) \\
\hline HD 1160 B & 2014.613 & $780.9 \pm 1.1$ & $244.25 \pm 0.13$ & Maire et al. (2016) \\
\hline HD $1160 \mathrm{~B}$ & 2014.613 & $781.0 \pm 0.5$ & $243.9 \pm 0.2$ & Maire et al. (2016) \\
\hline HD $1160 \mathrm{~B}$ & 2017.936 & $784 \pm 6$ & $244.9 \pm 0.3$ & Currie et al. (2018) \\
\hline HD 19467 B & 2011.660 & $1663 \pm 5$ & $243.14 \pm 0.19$ & Crepp et al. (2014) \\
\hline HD 19467 B & 2012.016 & $1666 \pm 7$ & $242.3 \pm 0.3$ & Crepp et al. (2014) \\
\hline HD 19467 B & 2012.016 & $1657 \pm 7$ & $242.4 \pm 0.4$ & Crepp et al. (2014) \\
\hline HD 19467 B & 2012.652 & $1662 \pm 4$ & $242.19 \pm 0.15$ & Crepp et al. (2014) \\
\hline HD 19467 B & 2012.758 & $1653 \pm 4$ & $242.13 \pm 0.14$ & Crepp et al. (2014) \\
\hline HD 19467 B & 2013.791 & $1640 \pm 7$ & $241.7 \pm 0.3$ & Crepp et al. (2015) \\
\hline 1RXS0342+1216 B & 2007.951 & $883.0 \pm 0.2$ & $17.58 \pm 0.09$ & Bowler et al. (2015b) \\
\hline $1 \mathrm{RXS} 0342+1216 \mathrm{~B}$ & 2008.63 & $860 \pm 8$ & $17.3 \pm 0.4$ & Janson et al. (2012) \\
\hline 1RXS0342+1216 B & 2008.87 & $866 \pm 8$ & $17.8 \pm 0.4$ & Janson et al. (2012) \\
\hline 1RXS0342+1216 B & 2010.659 & $851 \pm 3$ & $18.7 \pm 0.1$ & Bowler et al. (2015b) \\
\hline 1RXS0342+1216 B & 2012.02 & $834 \pm 57$ & $17.6 \pm 1.7$ & Janson et al. (2014) \\
\hline 1RXS0342+1216 B & 2012.645 & $831 \pm 2$ & $18.71 \pm 0.07$ & Bowler et al. (2015a) \\
\hline 1RXS0342+1216 B & 2013.044 & $822 \pm 8$ & $19.1 \pm 0.7$ & Bowler et al. (2015a) \\
\hline 51 Eri b & 2014.961 & $450 \pm 7$ & $171.0 \pm 0.9$ & De Rosa et al. (2015) \\
\hline 51 Eri b & 2015.079 & $454 \pm 6$ & $170.6 \pm 1.0$ & De Rosa et al. (2015) \\
\hline 51 Eri b & 2015.082 & $462 \pm 7$ & $170.5 \pm 0.9$ & De Rosa et al. (2015) \\
\hline 51 Eri b & 2015.085 & $462 \pm 24$ & $170.4 \pm 3$ & De Rosa et al. (2015) \\
\hline 51 Eri b & 2015.665 & $455 \pm 6$ & $166.5 \pm 0.6$ & De Rosa et al. (2015) \\
\hline 51 Eri b & 2015.74 & $453 \pm 5$ & $167.2 \pm 0.6$ & Maire et al. (2019) \\
\hline 51 Eri b & 2015.74 & $454 \pm 16$ & $166 \pm 2$ & Maire et al. (2019) \\
\hline 51 Eri b & 2016.04 & $457 \pm 7$ & $165.5 \pm 0.8$ & Maire et al. (2019) \\
\hline 51 Eri b & 2016.95 & $454 \pm 6$ & $160.3 \pm 0.7$ & Maire et al. (2019) \\
\hline 51 Eri b & 2017.74 & $449 \pm 3$ & $155.7 \pm 0.4$ & Maire et al. (2019) \\
\hline 51 Eri b & 2018.72 & $443 \pm 4$ & $150.2 \pm 0.6$ & Maire et al. (2019) \\
\hline CD-35 2722 B & 2009.041 & $3172 \pm 5$ & $244.1 \pm 0.3$ & Wahhaj et al. (2011) \\
\hline CD-35 2722 B & 2010.025 & $3137 \pm 5$ & $243.1 \pm 0.3$ & Wahhaj et al. (2011) \\
\hline HD 49197 B & 2002.158 & $950 \pm 5$ & $78.3 \pm 0.4$ & Metchev \& Hillenbrand (2004) \\
\hline HD 49197 B & 2003.854 & $948 \pm 2$ & $77.6 \pm 0.3$ & Metchev \& Hillenbrand (2004) \\
\hline HD 49197 B & 2006.690 & $960 \pm 100$ & $77 \pm 2$ & Serabyn et al. (2009) \\
\hline HD 49197 B & 2015.890 & $862 \pm 25$ & $76.6 \pm 1.8$ & Bottom et al. (2017) \\
\hline HR 2562 B & 2016.066 & $619 \pm 3$ & $297.6 \pm 0.4$ & Konopacky et al. (2016) \\
\hline HR 2562 B & 2016.066 & $618 \pm 4$ & $297.8 \pm 0.5$ & Konopacky et al. (2016) \\
\hline HR 2562 B & 2016.074 & $618 \pm 5$ & $297.4 \pm 0.4$ & Konopacky et al. (2016) \\
\hline HR 2562 B & 2016.151 & $619 \pm 2$ & $297.5 \pm 0.3$ & Konopacky et al. (2016) \\
\hline HR 2562 B & 2016.95 & $638 \pm 6$ & $297.8 \pm 0.5$ & Maire et al. (2018) \\
\hline HR 2562 B & 2017.10 & $644 \pm 2$ & $297.8 \pm 0.2$ & Maire et al. (2018) \\
\hline HR 2562 B & 2017.10 & $644 \pm 3$ & $297.5 \pm 0.3$ & Maire et al. (2018) \\
\hline HR 2562 B & 2017.75 & $661.2 \pm 1.3$ & $297.97 \pm 0.16$ & Maire et al. (2018) \\
\hline HR 2562 B & 2017.75 & $658.9 \pm 1.6$ & $298.08 \pm 0.17$ & Maire et al. (2018) \\
\hline HR 2562 B & 2017.75 & $658 \pm 3$ & $297.7 \pm 0.2$ & Maire et al. (2018) \\
\hline HR 3549 B & 2013.033 & $873 \pm 13$ & $157.6 \pm 0.6$ & Mawet et al. (2015) \\
\hline HR 3549 B & 2015.034 & $856 \pm 21$ & $157.0 \pm 1.0$ & Mawet et al. (2015) \\
\hline HR 3549 B & 2015.964 & $850 \pm 6$ & $155.8 \pm 0.5$ & Mesa et al. (2016) \\
\hline HR 3549 B & 2015.964 & $848 \pm 9$ & $156.1 \pm 0.7$ & Mesa et al. (2016) \\
\hline HD 95086 b & 2012.030 & $624 \pm 8$ & $151.9 \pm 0.8$ & Chauvin et al. (2018) \\
\hline HD 95086 b & 2013.197 & $626 \pm 13$ & $150.8 \pm 1.3$ & Chauvin et al. (2018) \\
\hline HD 95086 b & 2013.485 & $600 \pm 11$ & $151.0 \pm 1.2$ & Chauvin et al. (2018) \\
\hline HD 95086 b & 2013.939 & $619 \pm 5$ & $150.9 \pm 0.5$ & Rameau et al. (2016) \\
\hline HD 95086 b & 2013.942 & $618 \pm 11$ & $150.3 \pm 1.1$ & Rameau et al. (2016) \\
\hline HD 95086 b & 2014.361 & $618 \pm 8$ & $150.2 \pm 0.7$ & Rameau et al. (2016) \\
\hline HD 95086 b & 2015.090 & $622 \pm 4$ & $148.8 \pm 0.4$ & Chauvin et al. (2018) \\
\hline
\end{tabular}


Table 8

(Continued)

\begin{tabular}{|c|c|c|c|c|}
\hline Name & $\begin{array}{l}\text { Epoch } \\
\text { (UT) }\end{array}$ & $\begin{array}{l}\text { Separation } \\
\text { (mas) }\end{array}$ & $\begin{array}{l}\text { P.A. } \\
\text { (deg) }\end{array}$ & Reference \\
\hline HD 95086 b & 2015.090 & $620 \pm 5$ & $149.0 \pm 0.5$ & Chauvin et al. (2018) \\
\hline HD 95086 b & 2015.260 & $622 \pm 7$ & $148.8 \pm 0.6$ & Rameau et al. (2016) \\
\hline HD 95086 b & 2015.266 & $622 \pm 4$ & $149.0 \pm 0.4$ & Rameau et al. (2016) \\
\hline HD 95086 b & 2015.340 & $622 \pm 7$ & $148.6 \pm 0.6$ & Chauvin et al. (2018) \\
\hline HD 95086 b & 2015.340 & $620 \pm 8$ & $148.7 \pm 0.6$ & Chauvin et al. (2018) \\
\hline HD 95086 b & 2016.047 & $624 \pm 8$ & $148.4 \pm 0.7$ & Chauvin et al. (2018) \\
\hline HD 95086 b & 2016.047 & $626 \pm 10$ & $148.6 \pm 0.9$ & Chauvin et al. (2018) \\
\hline HD 95086 b & 2016.162 & $621 \pm 5$ & $147.8 \pm 0.5$ & Rameau et al. (2016) \\
\hline HD 95086 b & 2016.178 & $620 \pm 5$ & $147.2 \pm 0.5$ & Rameau et al. (2016) \\
\hline HD $95086 \mathrm{~b}$ & 2016.413 & $622 \pm 3$ & $147.5 \pm 0.3$ & Chauvin et al. (2018) \\
\hline HD 95086 b & 2016.413 & $620 \pm 4$ & $147.6 \pm 0.4$ & Chauvin et al. (2018) \\
\hline HD 95086 b & 2017.353 & $624 \pm 3$ & $146.6 \pm 0.3$ & Chauvin et al. (2018) \\
\hline HD 95086 b & 2017.353 & $626 \pm 4$ & $146.8 \pm 0.4$ & Chauvin et al. (2018) \\
\hline GJ $504 \mathrm{~B}$ & 2011.230 & $2479 \pm 16$ & $327.9 \pm 0.4$ & Kuzuhara et al. (2013) \\
\hline GJ $504 \mathrm{~B}$ & 2011.386 & $2483 \pm 8$ & $327.5 \pm 0.2$ & Kuzuhara et al. (2013) \\
\hline GJ $504 \mathrm{~B}$ & 2011.611 & $2481 \pm 33$ & $326.8 \pm 0.9$ & Kuzuhara et al. (2013) \\
\hline GJ 504 B & 2011.619 & $2448 \pm 24$ & $325.8 \pm 0.7$ & Kuzuhara et al. (2013) \\
\hline GJ $504 \mathrm{~B}$ & 2012.159 & $2483 \pm 15$ & $326.5 \pm 0.4$ & Kuzuhara et al. (2013) \\
\hline GJ 504 B & 2012.279 & $2487 \pm 8$ & $326.5 \pm 0.2$ & Kuzuhara et al. (2013) \\
\hline GJ 504 B & 2012.397 & $2499 \pm 26$ & $326.1 \pm 0.6$ & Kuzuhara et al. (2013) \\
\hline GJ 504 B & 2015.340 & $2491 \pm 3$ & $323.46 \pm 0.07$ & Bonnefoy et al. (2018) \\
\hline GJ $504 \mathrm{~B}$ & 2015.419 & $2496 \pm 3$ & $323.50 \pm 0.07$ & Bonnefoy et al. (2018) \\
\hline GJ 504 B & 2015.424 & $2497 \pm 4$ & $323.60 \pm 0.10$ & Bonnefoy et al. (2018) \\
\hline GJ $504 \mathrm{~B}$ & 2015.427 & $2495 \pm 5$ & $323.50 \pm 0.14$ & Bonnefoy et al. (2018) \\
\hline GJ 504 B & 2015.427 & $2501 \pm 3$ & $323.49 \pm 0.07$ & Bonnefoy et al. (2018) \\
\hline GJ 504 B & 2015.430 & $2499 \pm 6$ & $323.40 \pm 0.14$ & Bonnefoy et al. (2018) \\
\hline GJ 504 B & 2016.241 & $2495 \pm 2$ & $322.48 \pm 0.05$ & Bonnefoy et al. (2018) \\
\hline GJ 504 B & 2016.241 & $2493 \pm 12$ & $322.8 \pm 0.3$ & Bonnefoy et al. (2018) \\
\hline GJ 504 B & 2017.110 & $2493 \pm 3$ & $321.74 \pm 0.08$ & Bonnefoy et al. (2018) \\
\hline HIP 65426 b & 2016.411 & $830 \pm 5$ & $150.3 \pm 0.2$ & Chauvin et al. (2017) \\
\hline HIP 65426 b & 2016.485 & $830 \pm 3$ & $150.14 \pm 0.17$ & Chauvin et al. (2017) \\
\hline HIP 65426 b & 2017.101 & $827.6 \pm 1.5$ & $150.11 \pm 0.15$ & Chauvin et al. (2017) \\
\hline HIP 65426 b & 2017.107 & $828.8 \pm 1.5$ & $150.05 \pm 0.16$ & Chauvin et al. (2017) \\
\hline HIP 65426 b & 2017.375 & $832 \pm 3$ & $149.52 \pm 0.19$ & Cheetham et al. (2019) \\
\hline HIP 65426 b & 2017.378 & $850 \pm 20$ & $148.5 \pm 1.6$ & Cheetham et al. (2019) \\
\hline HIP 65426 b & 2018.359 & $823 \pm 2$ & $149.85 \pm 0.15$ & Cheetham et al. (2019) \\
\hline HIP 65426 b & 2018.359 & $826 \pm 2$ & $149.89 \pm 0.16$ & Cheetham et al. (2019) \\
\hline PDS $70 \mathrm{~b}$ & 2012.246 & $192 \pm 21$ & $162 \pm 4$ & Keppler et al. (2018) \\
\hline PDS $70 \mathrm{~b}$ & 2014.353 & $194 \pm 5$ & $159 \pm 3$ & Christiaens et al. (2019) \\
\hline PDS $70 \mathrm{~b}$ & 2015.334 & $192 \pm 4$ & $154.5 \pm 1.2$ & Keppler et al. (2018) \\
\hline PDS $70 \mathrm{~b}$ & 2015.334 & $197 \pm 4$ & $154.9 \pm 1.1$ & Keppler et al. (2018) \\
\hline PDS $70 \mathrm{~b}$ & 2015.411 & $200 \pm 7$ & $153.4 \pm 1.8$ & Keppler et al. (2018) \\
\hline PDS $70 \mathrm{~b}$ & 2015.411 & $195 \pm 6$ & $153.5 \pm 1.8$ & Keppler et al. (2018) \\
\hline PDS $70 \mathrm{~b}$ & 2016.367 & $186 \pm 7$ & $152.4 \pm 1.5$ & Haffert et al. (2019) \\
\hline PDS $70 \mathrm{~b}$ & 2016.367 & $199 \pm 7$ & $151.5 \pm 1.6$ & Keppler et al. (2018) \\
\hline PDS $70 \mathrm{~b}$ & 2016.416 & $181 \pm 10$ & $151 \pm 2$ & Haffert et al. (2019) \\
\hline PDS $70 \mathrm{~b}$ & 2018.148 & $192 \pm 8$ & $147.0 \pm 2.4$ & Müller et al. (2018) \\
\hline PDS $70 \mathrm{~b}$ & 2018.148 & $192 \pm 8$ & $146.8 \pm 2.4$ & Müller et al. (2018) \\
\hline PDS $70 \mathrm{~b}$ & 2018.334 & $183 \pm 18$ & $148.8 \pm 1.7$ & Wagner et al. (2018) \\
\hline PDS $70 \mathrm{~b}$ & 2018.337 & $193 \pm 12$ & $143.4 \pm 4.2$ & Wagner et al. (2018) \\
\hline PDS $70 \mathrm{~b}$ & 2018.465 & $177 \pm 25$ & $146.8 \pm 8.5$ & Haffert et al. (2019) \\
\hline PZ Tel B & 2007.446 & $255 \pm 3$ & $61.7 \pm 0.6$ & Mugrauer et al. (2012) \\
\hline PZ Tel B & 2009.739 & $336.6 \pm 1.2$ & $60.5 \pm 0.2$ & Mugrauer et al. (2012) \\
\hline PZ Tel B & 2010.274 & $330 \pm 10$ & $59.0 \pm 1.0$ & Biller et al. (2010) \\
\hline PZ Tel B & 2010.340 & $356.4 \pm 1.1$ & $60.3 \pm 0.2$ & Mugrauer et al. (2012) \\
\hline PZ Tel B & 2010.345 & $354.7 \pm 1.2$ & $60.3 \pm 0.2$ & Mugrauer et al. (2012) \\
\hline PZ Tel B & 2010.350 & $360 \pm 3$ & $59.4 \pm 0.5$ & Biller et al. (2010) \\
\hline PZ Tel B & 2010.734 & $365 \pm 8$ & $59.2 \pm 0.8$ & Beust et al. (2016) \\
\hline PZ Tel B & 2010.821 & $369.3 \pm 1.1$ & $59.9 \pm 0.2$ & Mugrauer et al. (2012) \\
\hline PZ Tel B & 2011.227 & $382.2 \pm 1.0$ & $59.8 \pm 0.2$ & Mugrauer et al. (2012) \\
\hline PZ Tel B & 2011.334 & $394 \pm 2$ & $60.4 \pm 0.2$ & Beust et al. (2016) \\
\hline PZ Tel B & 2011.419 & $387.8 \pm 1.2$ & $59.7 \pm 0.2$ & Mugrauer et al. (2012) \\
\hline PZ Tel B & 2011.422 & $388.5 \pm 0.8$ & $59.66 \pm 0.16$ & Mugrauer et al. (2012) \\
\hline PZ Tel B & 2011.424 & $387.1 \pm 1.4$ & $59.7 \pm 0.3$ & Mugrauer et al. (2012) \\
\hline PZ Tel B & 2011.427 & $389.0 \pm 1.0$ & $59.7 \pm 0.2$ & Mugrauer et al. (2012) \\
\hline PZ Tel B & 2011.430 & $390 \pm 5$ & $60.0 \pm 0.6$ & Beust et al. (2016) \\
\hline PZ Tel B & 2012.435 & $420.1 \pm 1.3$ & $59.6 \pm 0.2$ & Ginski et al. (2014) \\
\hline PZ Tel B & 2012.435 & $418.8 \pm 1.4$ & $59.6 \pm 0.2$ & Ginski et al. (2014) \\
\hline PZ Tel B & 2014.53 & $478.2 \pm 0.7$ & $59.71 \pm 0.19$ & Maire et al. (2016) \\
\hline PZ Tel B & 2014.53 & $478 \pm 2$ & $59.6 \pm 0.5$ & Maire et al. (2016) \\
\hline PZ Tel B & 2014.53 & $476 \pm 2$ & $60.1 \pm 0.5$ & Maire et al. (2016) \\
\hline
\end{tabular}


Table 8

(Continued)

\begin{tabular}{|c|c|c|c|c|}
\hline Name & $\begin{array}{l}\text { Epoch } \\
\text { (UT) }\end{array}$ & $\begin{array}{l}\text { Separation } \\
\quad \text { (mas) }\end{array}$ & $\begin{array}{l}\text { P.A. } \\
\text { (deg) }\end{array}$ & Reference \\
\hline$\overline{\text { PZ Tel B }}$ & 2014.60 & $479.5 \pm 0.7$ & $59.62 \pm 0.14$ & Maire et al. (2016) \\
\hline PZ Tel B & 2014.60 & $479.7 \pm 0.3$ & $59.7 \pm 0.5$ & Maire et al. (2016) \\
\hline PZ Tel B & 2014.60 & $479.6 \pm 0.3$ & $60.2 \pm 0.5$ & Maire et al. (2016) \\
\hline PZ Tel B & 2014.78 & $482.6 \pm 0.9$ & $59.44 \pm 0.15$ & Maire et al. (2016) \\
\hline PZ Tel B & 2014.78 & $483.9 \pm 0.3$ & $59.49 \pm 0.16$ & Maire et al. (2016) \\
\hline PZ Tel B & 2014.78 & $483.9 \pm 0.3$ & $59.51 \pm 0.16$ & Maire et al. (2016) \\
\hline HD 206893 B & 2015.758 & $270 \pm 3$ & $70.0 \pm 0.6$ & Milli et al. (2017) \\
\hline HD 206893 В & 2016.602 & $269 \pm 10$ & $61.6 \pm 1.9$ & Milli et al. (2017) \\
\hline HD 206893 B & 2016.709 & $265 \pm 2$ & $62.25 \pm 0.11$ & Delorme et al. (2017) \\
\hline HD 206893 в & 2017.531 & $260 \pm 2$ & $54.2 \pm 0.4$ & Grandjean et al. (2019) \\
\hline HD 206893 B & 2018.465 & $249.1 \pm 1.6$ & $45.5 \pm 0.4$ & Grandjean et al. (2019) \\
\hline$\kappa$ And $\mathrm{B}$ & 2012.000 & $1070 \pm 10$ & $55.7 \pm 0.6$ & Carson et al. (2013) \\
\hline$\kappa$ And B & 2012.518 & $1058 \pm 7$ & $56.0 \pm 0.4$ & Carson et al. (2013) \\
\hline$\kappa$ And B & 2012.841 & $1028 \pm 14$ & $55.4 \pm 0.6$ & Currie et al. (2018) \\
\hline$\kappa$ And B & 2013.627 & $1015 \pm 14$ & $54.8 \pm 0.6$ & Currie et al. (2018) \\
\hline$\kappa$ And B & 2017.676 & $914 \pm 17$ & $50.9 \pm 0.7$ & Currie et al. (2018) \\
\hline$\kappa$ And B & 2017.936 & $909 \pm 14$ & $50.3 \pm 0.6$ & Currie et al. (2018) \\
\hline HD 23514 B & 2006.939 & $2640 \pm 20$ & $228.7 \pm 1.0$ & Rodriguez et al. (2012) \\
\hline HD 23514 B & 2007.813 & $2640 \pm 10$ & $227.8 \pm 0.3$ & Rodriguez et al. (2012) \\
\hline HD 23514 B & 2008.843 & $2620 \pm 40$ & $227.2 \pm 0.5$ & Rodriguez et al. (2012) \\
\hline HD 23514 B & 2009.832 & $2642 \pm 3$ & $227.51 \pm 0.04$ & Rodriguez et al. (2012) \\
\hline HD 23514 B & 2009.835 & $2642 \pm 1$ & $227.7 \pm 0.03$ & Rodriguez et al. (2012) \\
\hline HD 23514 B & 2010.827 & $2644 \pm 4$ & $227.5 \pm 0.1$ & Rodriguez et al. (2012) \\
\hline HD 23514 B & 2010.827 & $2644 \pm 2$ & $227.48 \pm 0.05$ & Rodriguez et al. (2012) \\
\hline HD 23514 B & 2010.827 & $2642 \pm 0.5$ & $227.47 \pm 0.09$ & Rodriguez et al. (2012) \\
\hline HD 23514 B & 2010.827 & $2645 \pm 2$ & $227.52 \pm 0.02$ & Rodriguez et al. (2012) \\
\hline HD 23514 B & 2010.914 & $2646 \pm 33$ & $227.6 \pm 0.7$ & Yamamoto et al. (2013) \\
\hline DH Tau B & 1999.044 & $2332 \pm 10$ & $138.68 \pm 0.19$ & Ginski et al. (2014) \\
\hline DH Tau B & 2002.893 & $2340 \pm 6$ & $139.56 \pm 0.17$ & Itoh et al. (2005) \\
\hline DH Tau B & 2004.019 & $2344 \pm 3$ & $139.83 \pm 0.06$ & Itoh et al. (2005) \\
\hline DH Tau B & 2009.747 & $2339 \pm 4$ & $138.63 \pm 0.14$ & Ginski et al. (2014) \\
\hline DH Tau B & 2012.058 & $2332 \pm 6$ & $138.76 \pm 0.16$ & Ginski et al. (2014) \\
\hline DH Tau B & 2012.928 & $2343 \pm 6$ & $138.61 \pm 0.15$ & Ginski et al. (2014) \\
\hline DH Tau B & 2014.934 & $2343 \pm 1$ & $140.25 \pm 0.02$ & Bryan et al. (2016a) \\
\hline DH Tau B & 2015.844 & $2339 \pm 1$ & $139.94 \pm 0.02$ & Bryan et al. (2016a) \\
\hline Ross 458 B & 2000.134 & $475.1 \pm 7.1$ & $81.4 \pm 2.8$ & Mann et al. (2019) \\
\hline Ross 458 B & 2001.337 & $526.3 \pm 8.2$ & $66.9 \pm 2.6$ & Mann et al. (2019) \\
\hline Ross 458 B & 2001.515 & $522.0 \pm 6.2$ & $65.3 \pm 2.5$ & Mann et al. (2019) \\
\hline Ross 458 B & 2001.591 & $527.6 \pm 5.0$ & $64.1 \pm 2.6$ & Mann et al. (2019) \\
\hline Ross $458 \mathrm{~B}$ & 2002.170 & $533 \pm 12$ & $56.2 \pm 1.2$ & Mann et al. (2019) \\
\hline Ross 458 B & 2005.329 & $280 \pm 50$ & $357 \pm 1$ & Ward-Duong et al. (2015) \\
\hline Ross 458 B & 2006.389 & $236.2 \pm 3.9$ & $304.62 \pm 0.96$ & Mann et al. (2019) \\
\hline Ross 458 B & 2006.389 & $233.6 \pm 5.7$ & $304.3 \pm 1.5$ & Mann et al. (2019) \\
\hline Ross 458 B & 2007.142 & $270.6 \pm 8.2$ & $269.3 \pm 1.1$ & Mann et al. (2019) \\
\hline Ross 458 B & 2009.323 & $309.38 \pm 0.67$ & $203.182 \pm 0.069$ & Mann et al. (2019) \\
\hline Ross 458 B & 2009.323 & $307.5 \pm 1.1$ & $202.950 \pm 0.049$ & Mann et al. (2019) \\
\hline Ross 458 B & 2009.323 & $308.2 \pm 1.8$ & $203.22 \pm 0.10$ & Mann et al. (2019) \\
\hline Ross 458 B & 2013.301 & $448.30 \pm 0.66$ & $86.777 \pm 0.041$ & Mann et al. (2019) \\
\hline Ross 458 B & 2015.471 & $524.49 \pm 0.27$ & $60.087 \pm 0.016$ & Mann et al. (2019) \\
\hline $2 \mathrm{M} 1559+4403$ B & 2008.24 & $5654 \pm 4$ & $284.2 \pm 0.3$ & Janson et al. (2012) \\
\hline $2 \mathrm{M} 1559+4403$ B & 2009.13 & $5623 \pm 4$ & $284.9 \pm 0.3$ & Janson et al. (2012) \\
\hline $2 \mathrm{M} 1559+4403 \mathrm{~B}$ & 2009.42 & $5638 \pm 4$ & $284.8 \pm 0.3$ & Janson et al. (2012) \\
\hline $2 \mathrm{M} 1559+4403$ B & 2012.02 & $5598 \pm 56$ & $284.7 \pm 0.3$ & Janson et al. (2014) \\
\hline 2M1559+4403 B & 2012.357 & $5647 \pm 15$ & $284.46 \pm 0.10$ & Bowler et al. (2015a) \\
\hline $2 \mathrm{M} 1559+4403$ B & 2014.446 & $5670 \pm 70$ & $284.4 \pm 0.6$ & Bowler et al. (2015b) \\
\hline TWA 5 B & 1998.312 & $1960 \pm 10$ & $1.8 \pm 0.4$ & Lowrance et al. (1999) \\
\hline TWA 5 B & 2000.15 & $1954 \pm 8$ & $359.16 \pm 0.08$ & Brandeker et al. (2003) \\
\hline TWA 5 B & 2005.126 & $1940 \pm 20$ & $357.4 \pm 0.6$ & Galicher et al. (2016) \\
\hline TWA 5 B & 2007.518 & $1902 \pm 2$ & $356.4 \pm 0.2$ & Köhler et al. (2013) \\
\hline TWA 5 B & 2010.11 & $1897 \pm 11$ & $354.6 \pm 0.3$ & Janson et al. (2012) \\
\hline TWA 5 B & 2011.071 & $1888 \pm 7$ & $355.2 \pm 0.2$ & Köhler et al. (2013) \\
\hline TWA 5 B & 2012.003 & $1879 \pm 2$ & $355.0 \pm 0.1$ & Köhler et al. (2013) \\
\hline TWA 5 B & 2012.01 & $1869 \pm 19$ & $355.1 \pm 0.3$ & Janson et al. (2014) \\
\hline TWA 5 B & 2012.049 & $1875 \pm 3$ & $354.8 \pm 0.1$ & Köhler et al. (2013) \\
\hline TWA 5 B & 2013.044 & $1873 \pm 2$ & $354.5 \pm 0.1$ & Köhler et al. (2013) \\
\hline 1RXS2351+3127 B & 2011.470 & $2392.2 \pm 2.0$ & $91.77 \pm 0.05$ & Bowler et al. (2012) \\
\hline 1RXS2351+3127 B & 2011.871 & $2386.3 \pm 1.5$ & $91.81 \pm 0.04$ & Bowler et al. (2012) \\
\hline 1RXS2351+3127 B & 2013.626 & $2391 \pm 4$ & $91.63 \pm 0.02$ & Bowler et al. (2015a) \\
\hline 1RXS2351+3127 B & 2013.626 & $2391 \pm 3$ & $91.647 \pm 0.015$ & Bowler et al. (2015a) \\
\hline 1RXS2351+3127 B & 2013.626 & $2390.7 \pm 1.1$ & $91.65 \pm 0.01$ & Bowler et al. (2015a) \\
\hline 1RXS2351+3127 B & 2013.626 & $2391.2 \pm 1.1$ & $91.63 \pm 0.03$ & Bowler et al. (2015a) \\
\hline
\end{tabular}


Table 8

(Continued)

\begin{tabular}{|c|c|c|c|c|}
\hline Name & $\begin{array}{l}\text { Epoch } \\
\text { (UT) }\end{array}$ & $\begin{array}{l}\text { Separation } \\
\text { (mas) }\end{array}$ & $\begin{array}{l}\text { P.A. } \\
\text { (deg) }\end{array}$ & Reference \\
\hline 1RXS2351+3127 B & 2013.626 & $2391.6 \pm 1.7$ & $91.643 \pm 0.014$ & Bowler et al. (2015a) \\
\hline $1 \mathrm{RXS} 2351+3127 \mathrm{~B}$ & 2013.626 & $2390 \pm 5$ & $91.64 \pm 0.08$ & Bowler et al. (2015a) \\
\hline
\end{tabular}

\section{Appendix B}

\section{Corner Plots}

In this section we report corner plots displaying the joint and marginalized distributions of orbital elements from our orbit fits using orbitize! (Figures 26-47). These are also summarized in Table 3.

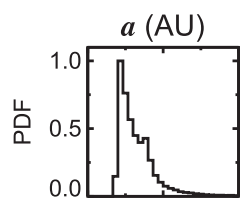

\section{HD 49197 B}
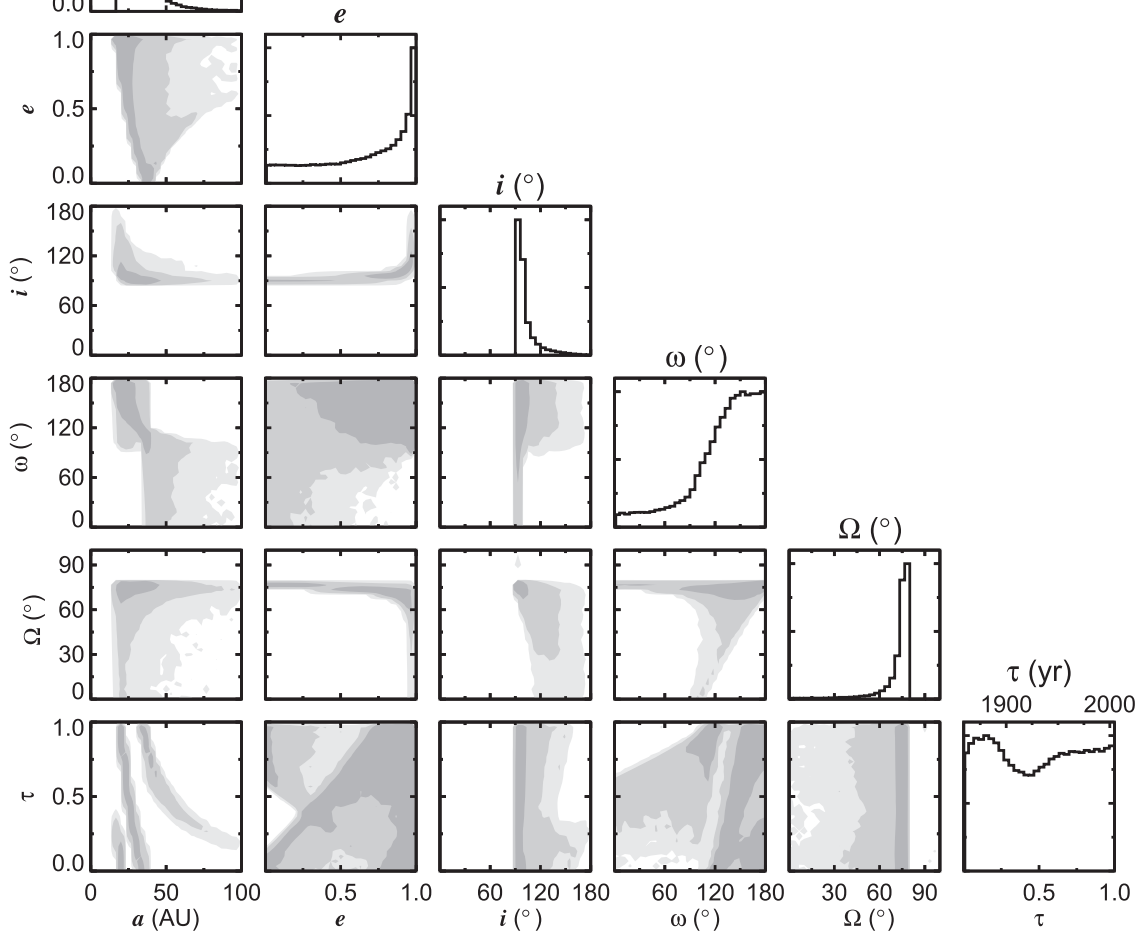

Figure 26. Corner plot for HD 49197 B. One-dimensional marginalized distributions are shown along the diagonal. Inclination $(i)$, argument of periastron $(\omega)$, and longitude of ascending node $(\Omega)$ are expressed in degrees. Units for the time of periastron passage, $\tau$, are fraction of the orbital period past MJD $=0$. Gray contours show the 1,2 , and $3 \sigma$ regions encompassing the two-dimensional joint posterior distributions. 


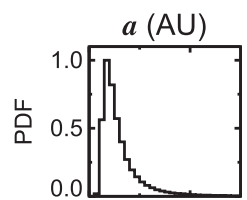

CD 35-2722 B
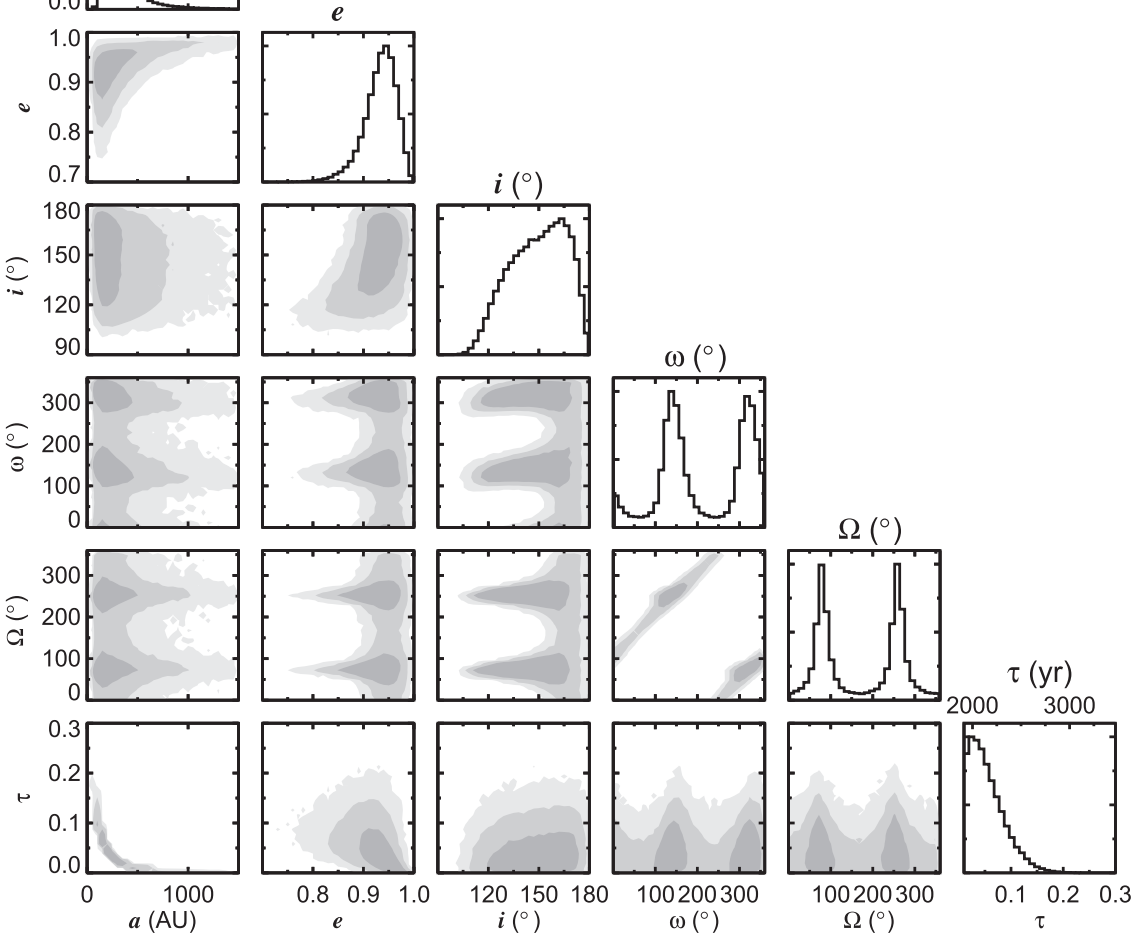

Figure 27. Corner plot for CD-35 2722 B. See Figure 26 for details.

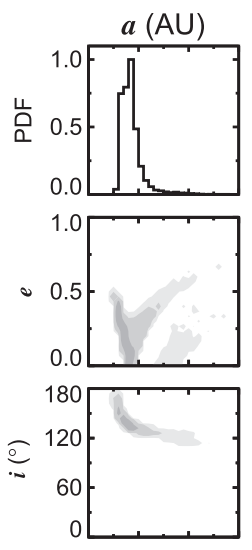

GJ 504 B
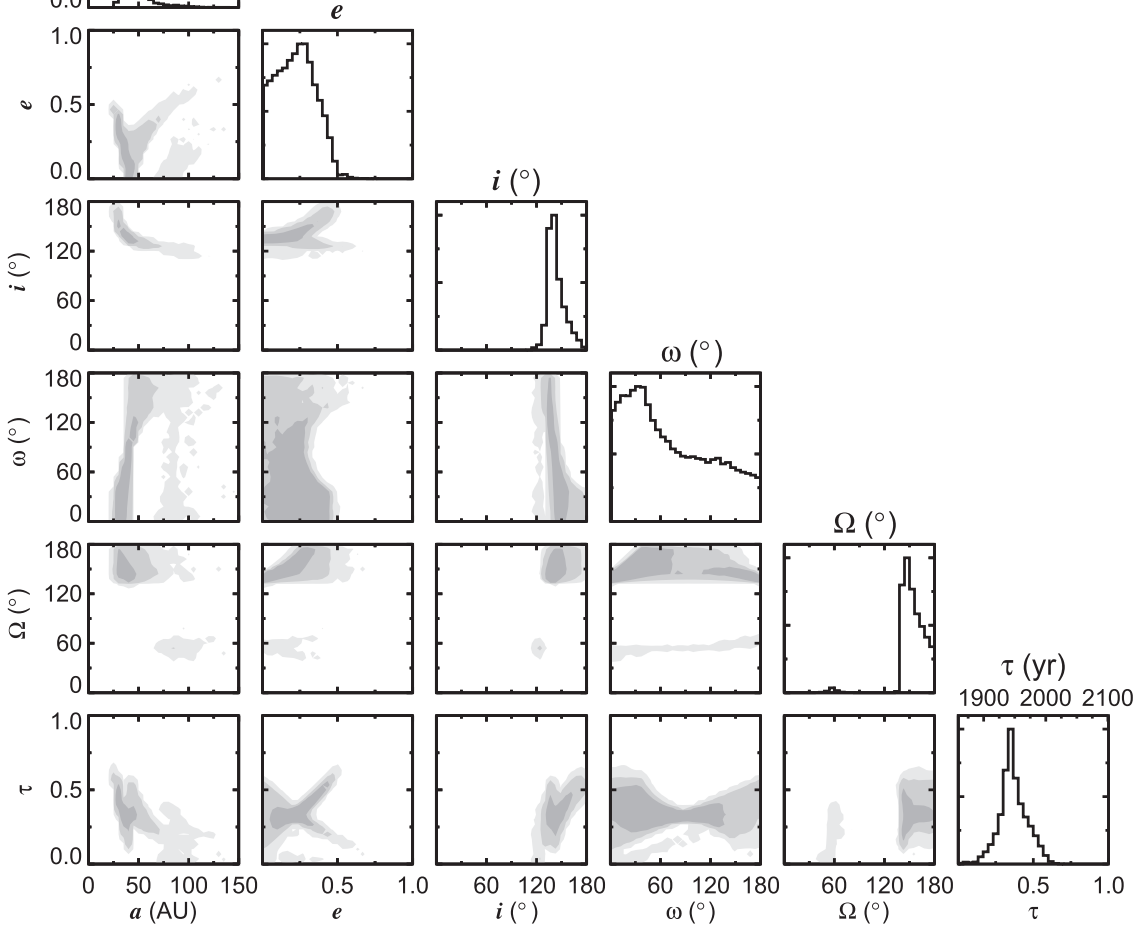

Figure 28. Corner plot for GJ 504 B. See Figure 26 for details. 


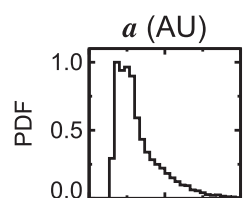

HD 19467 B
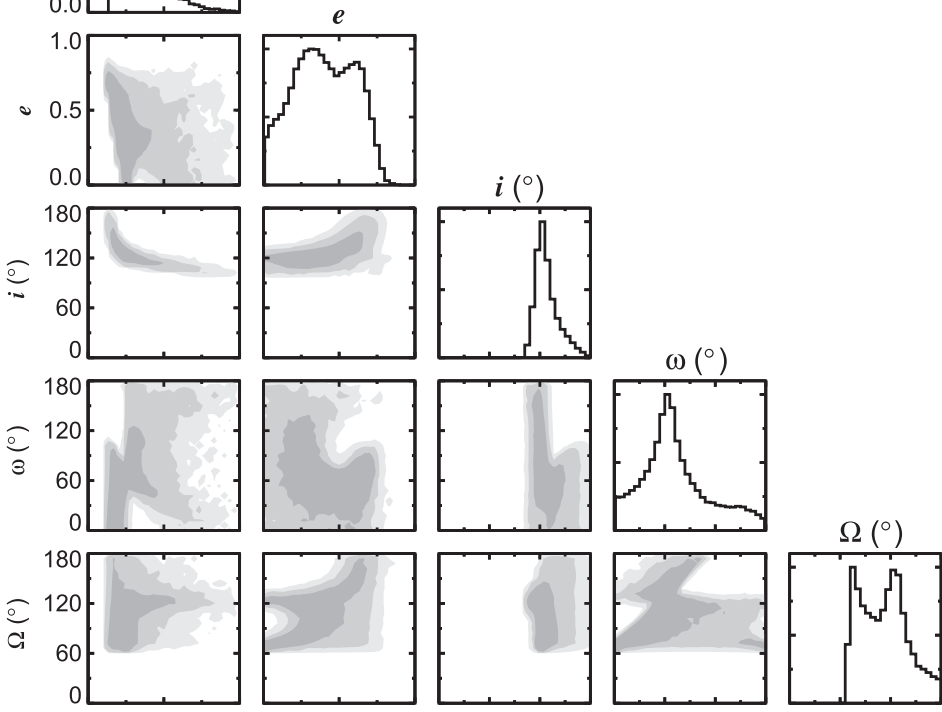

$\tau(\mathrm{yr})$
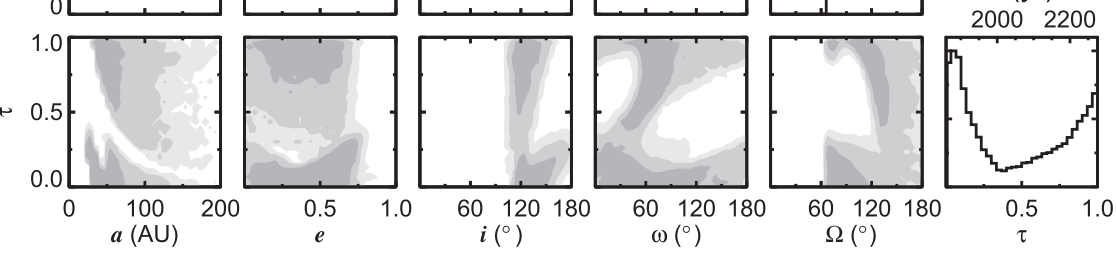

Figure 29. Corner plot for HD 19467 B. See Figure 26 for details.

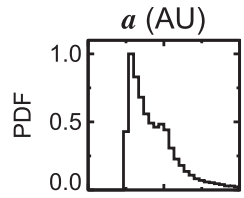

HD $1160 \mathrm{~B}$
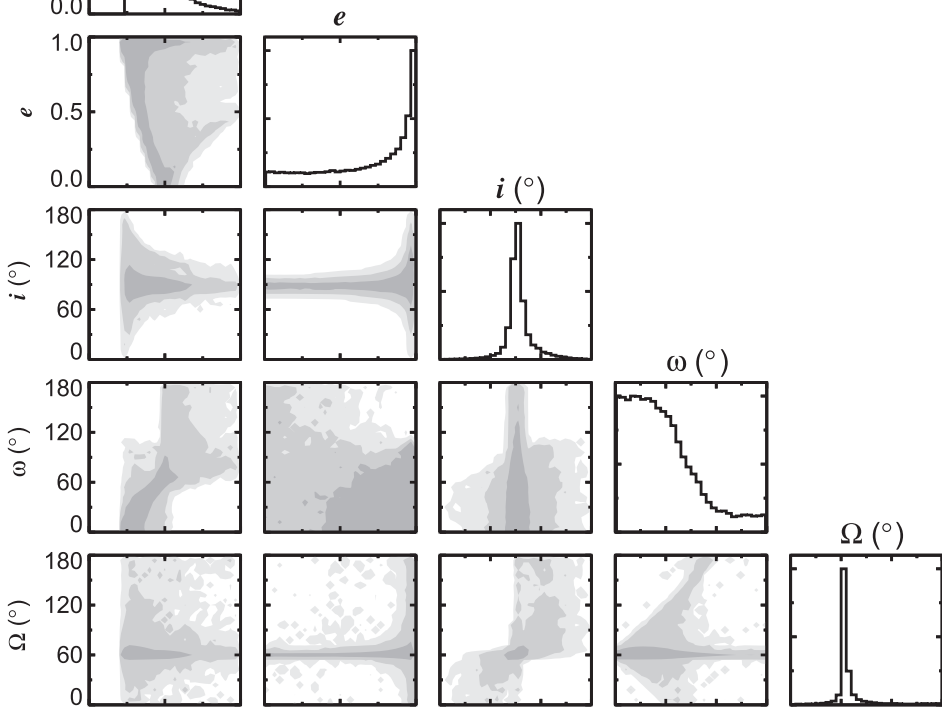

$\tau(\mathrm{yr})$
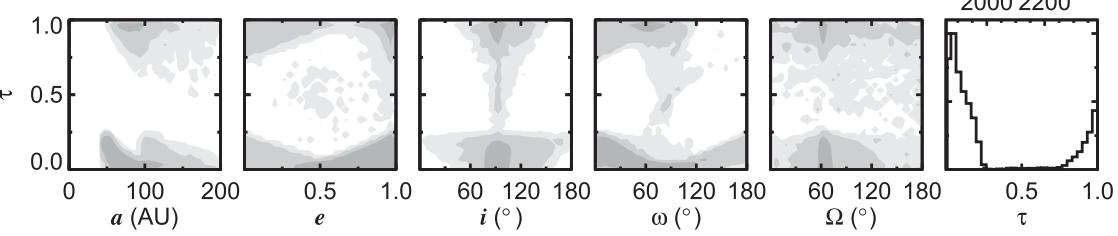

Figure 30. Corner plot for HD 1160 B. See Figure 26 for details. 


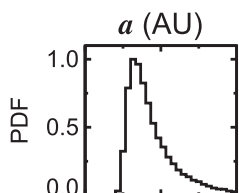

\section{$\kappa$ And $B$}
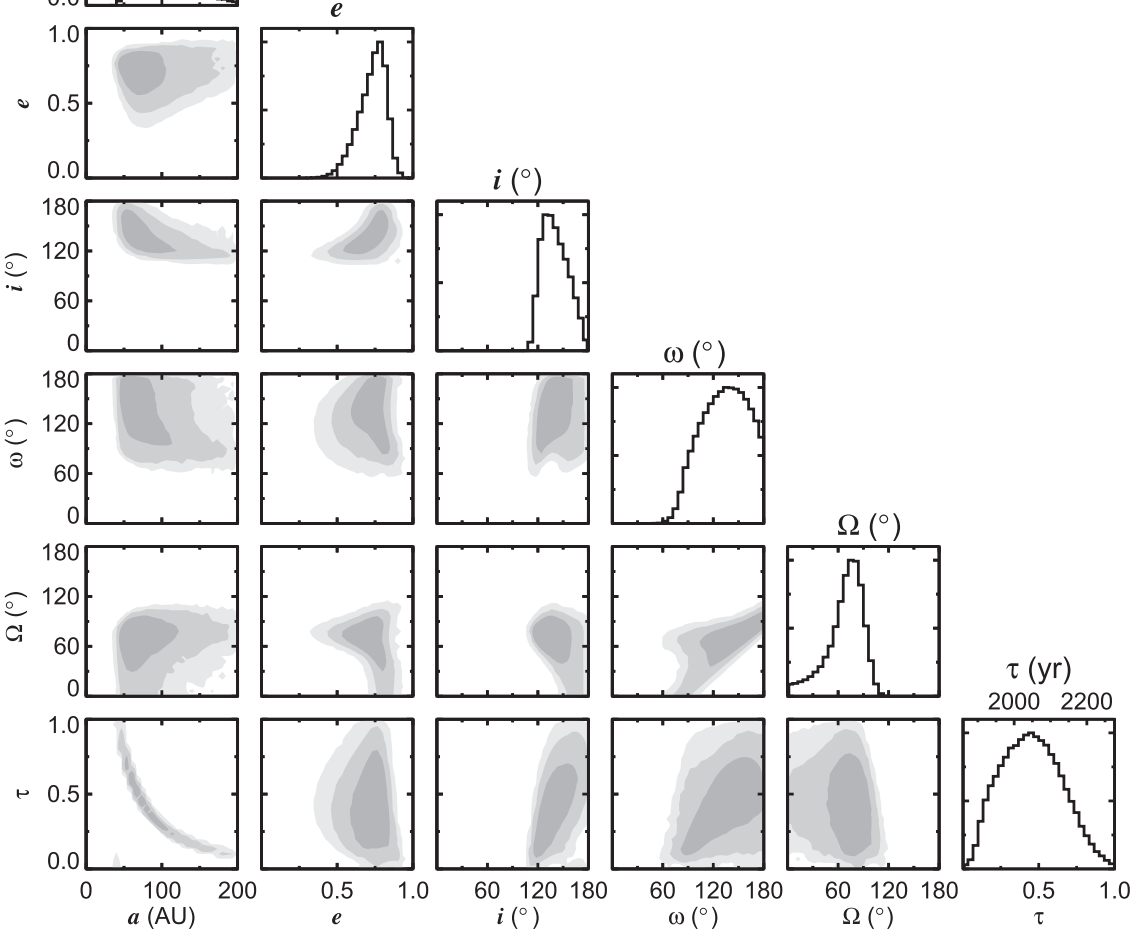

Figure 31. Corner plot for $\kappa$ And B. See Figure 26 for details.

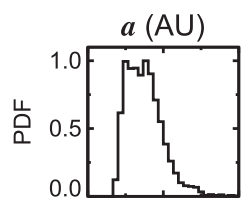

1RXS0342+1216 B
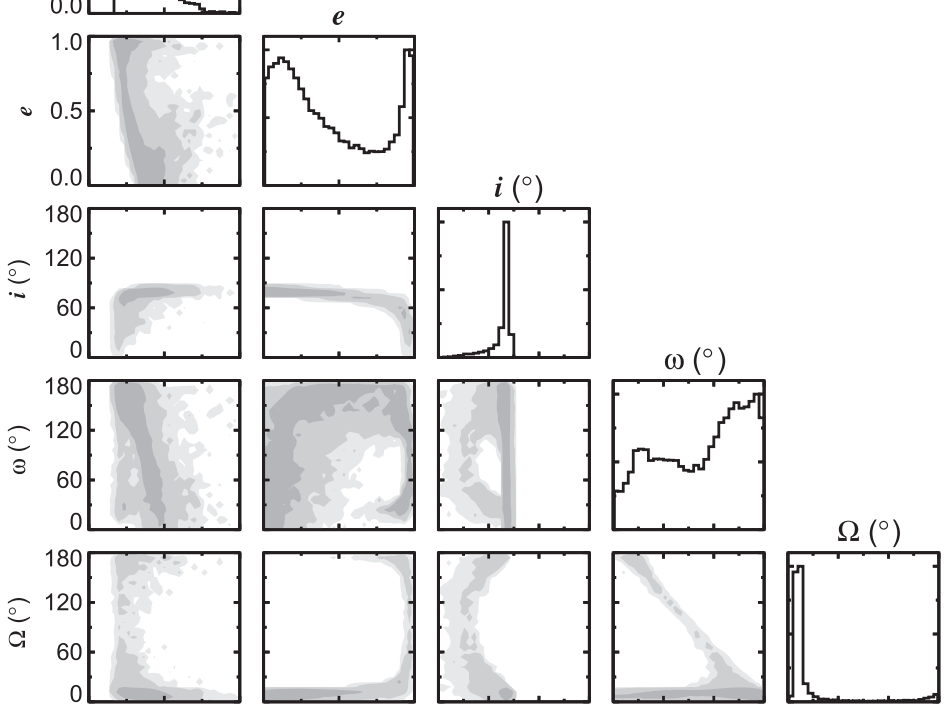

$\tau(\mathrm{yr})$
20002200
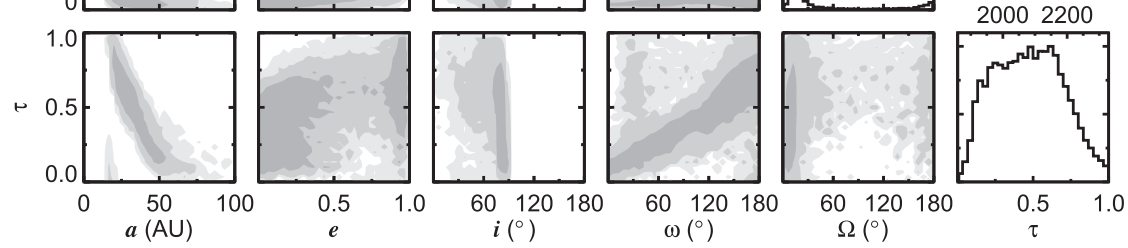

Figure 32. Corner plot for 1 RXS $0342+1216$ B. See Figure 26 for details. 


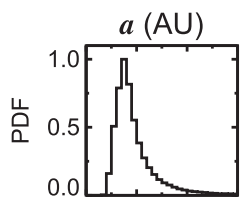

\section{HD 23514 B}
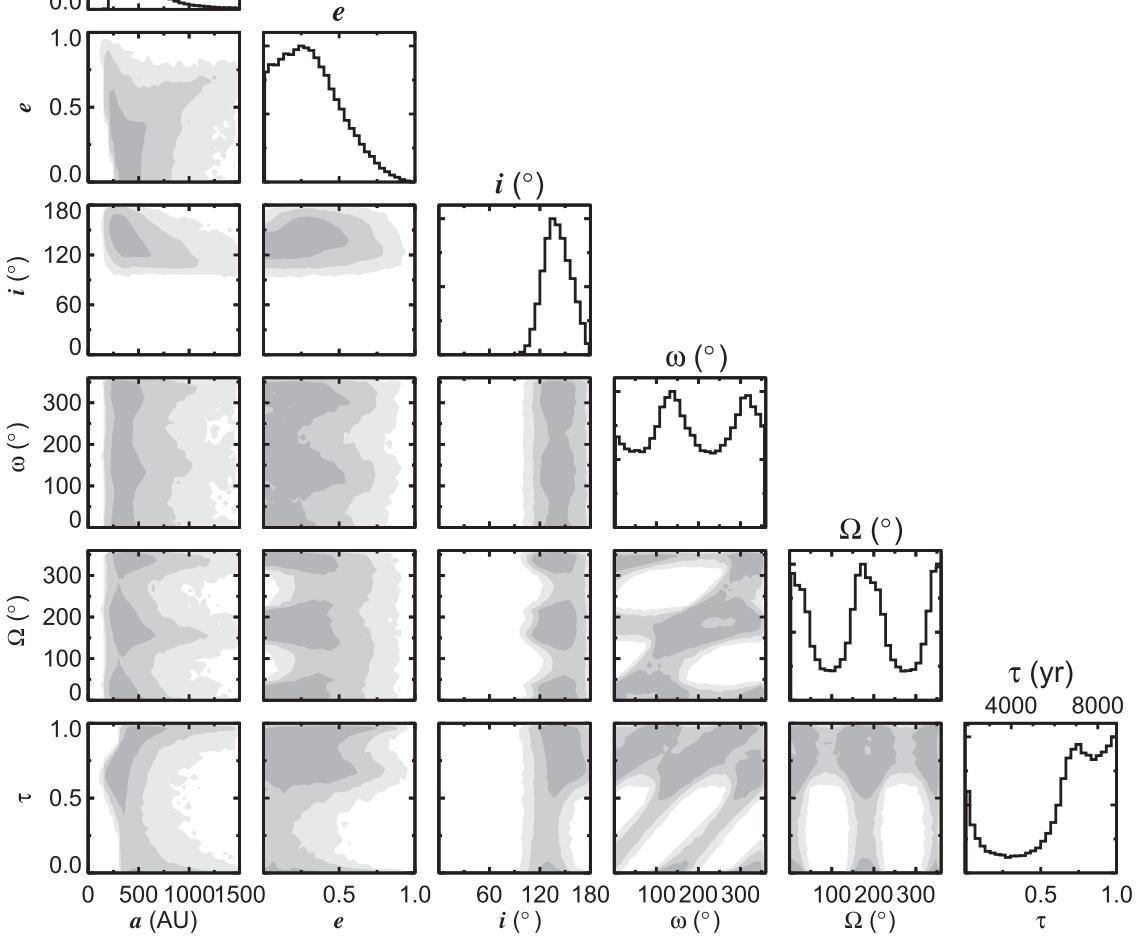

Figure 33. Corner plot for HD 23514 B. See Figure 26 for details.

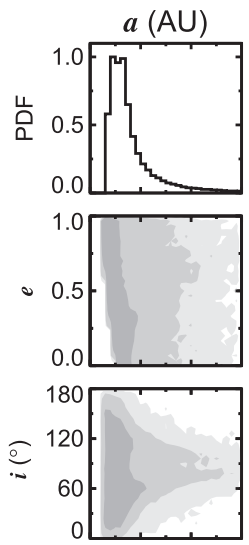

DH Tau B
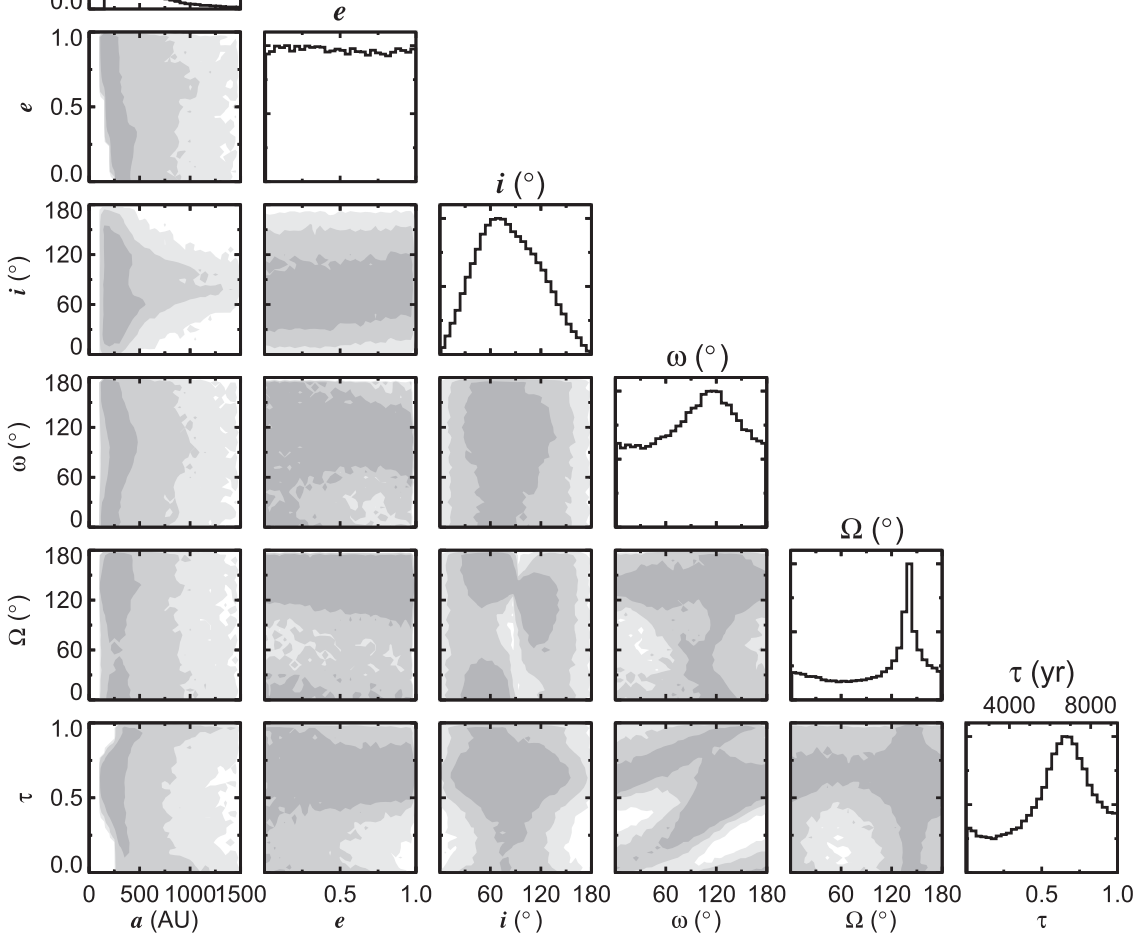

Figure 34. Corner plot for DH Tau B. See Figure 26 for details. 


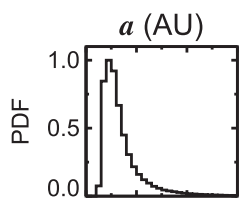

$2 \mathrm{M} 1559+4403 \mathrm{~B}$
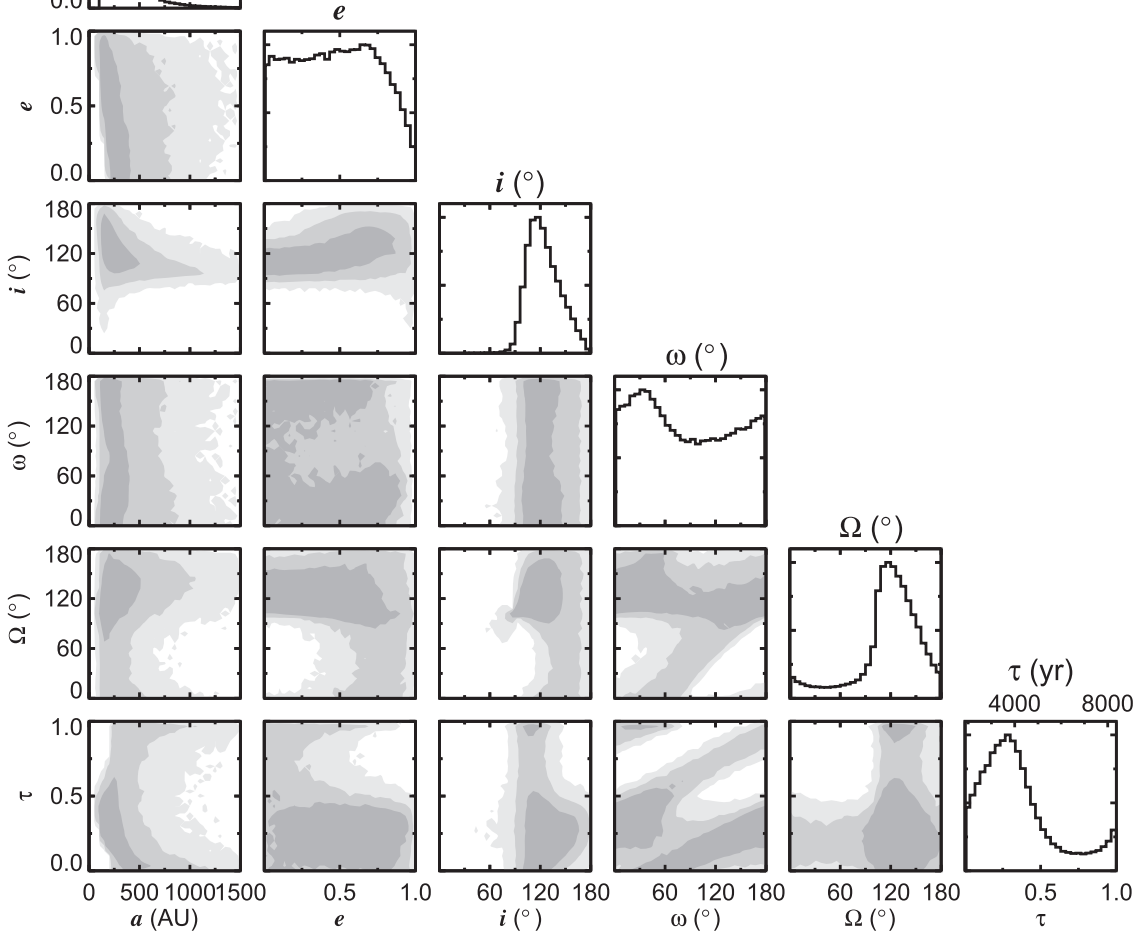

Figure 35. Corner plot for $2 \mathrm{M} 1559+4403$ B. See Figure 26 for details.
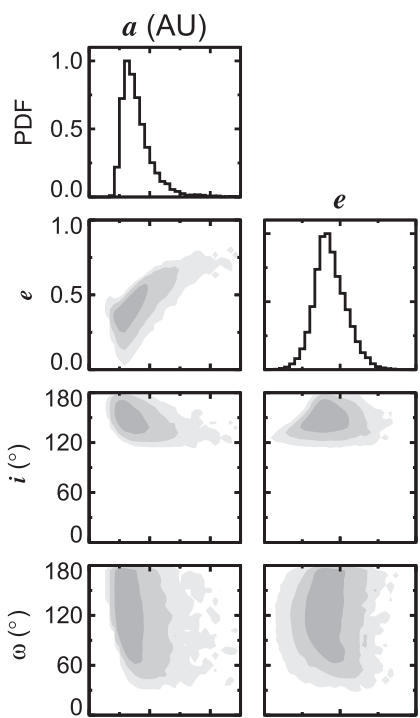

TWA 5 B
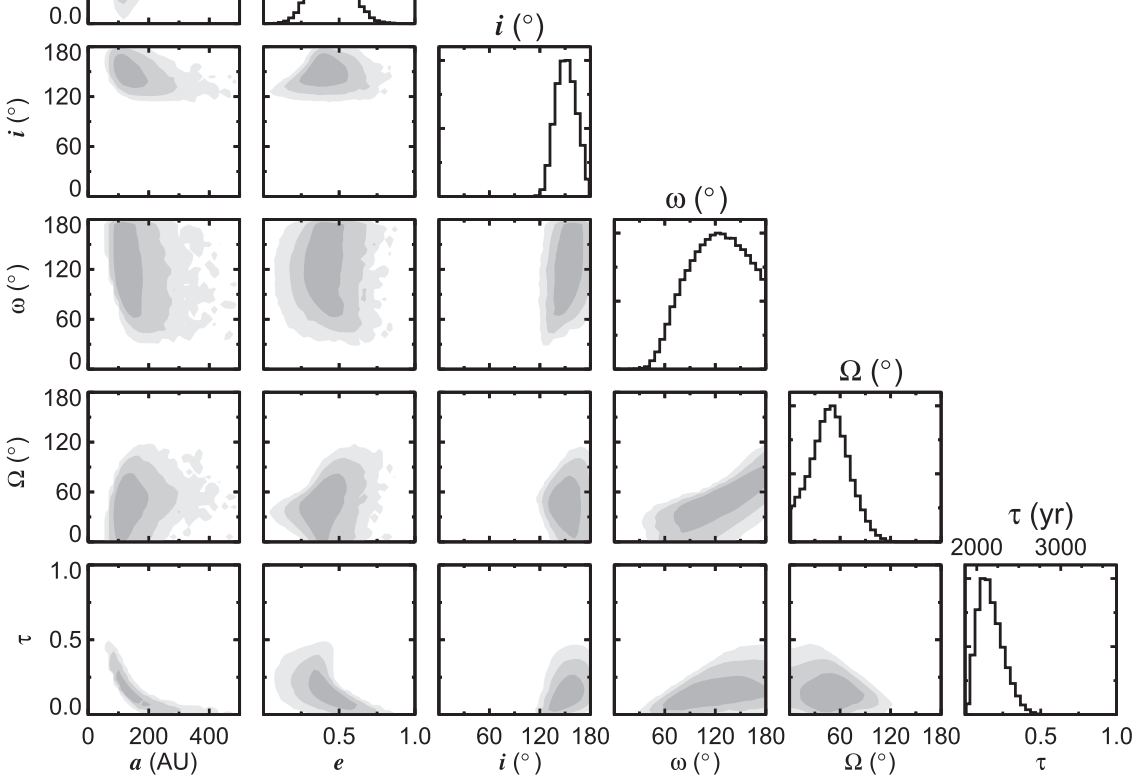

Figure 36. Corner plot for TWA 5 B. See Figure 26 for details. 


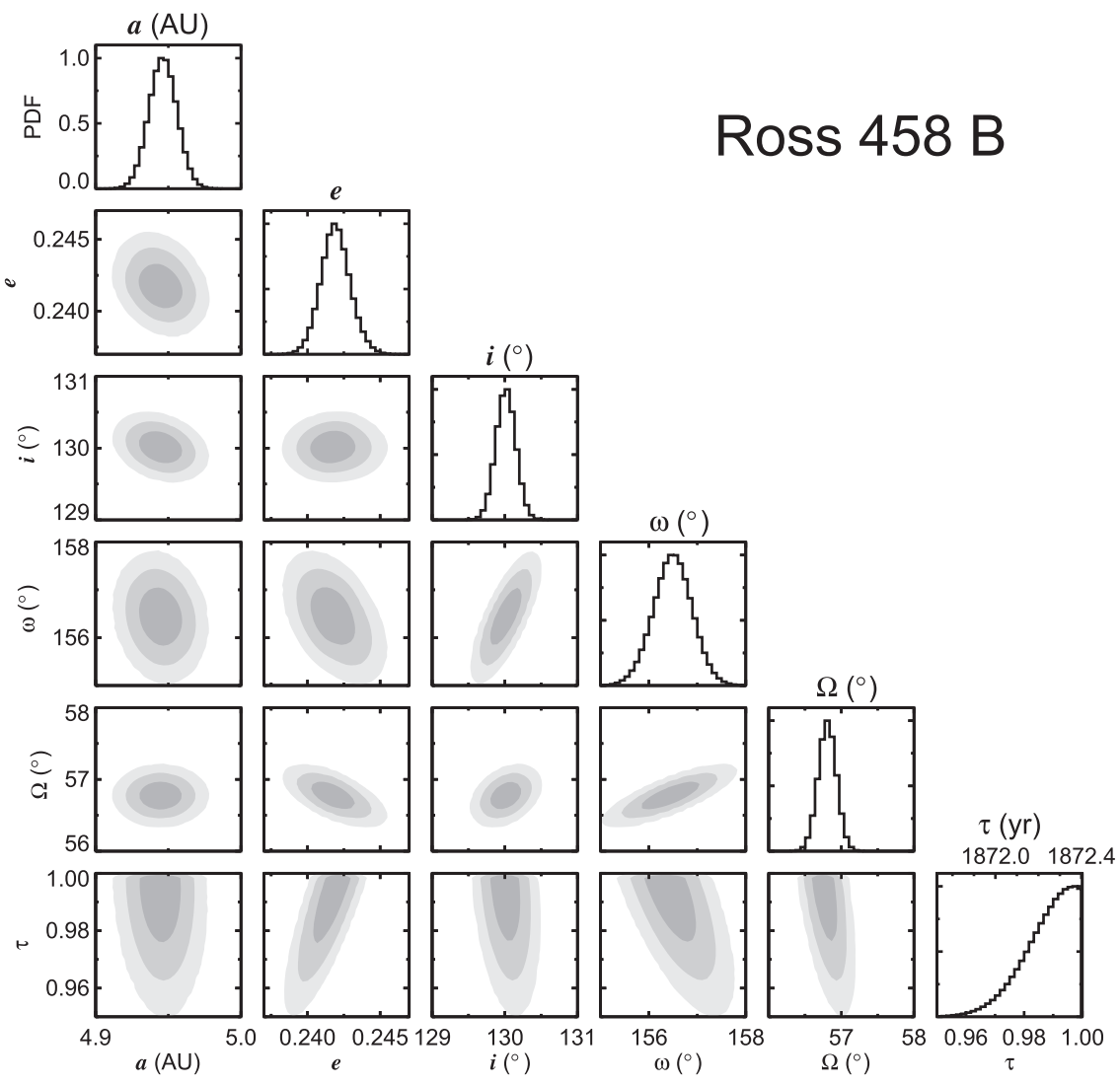

Figure 37. Corner plot for Ross 458 B. See Figure 26 for details.

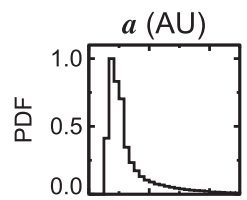

\section{RXS2351+3127 B}
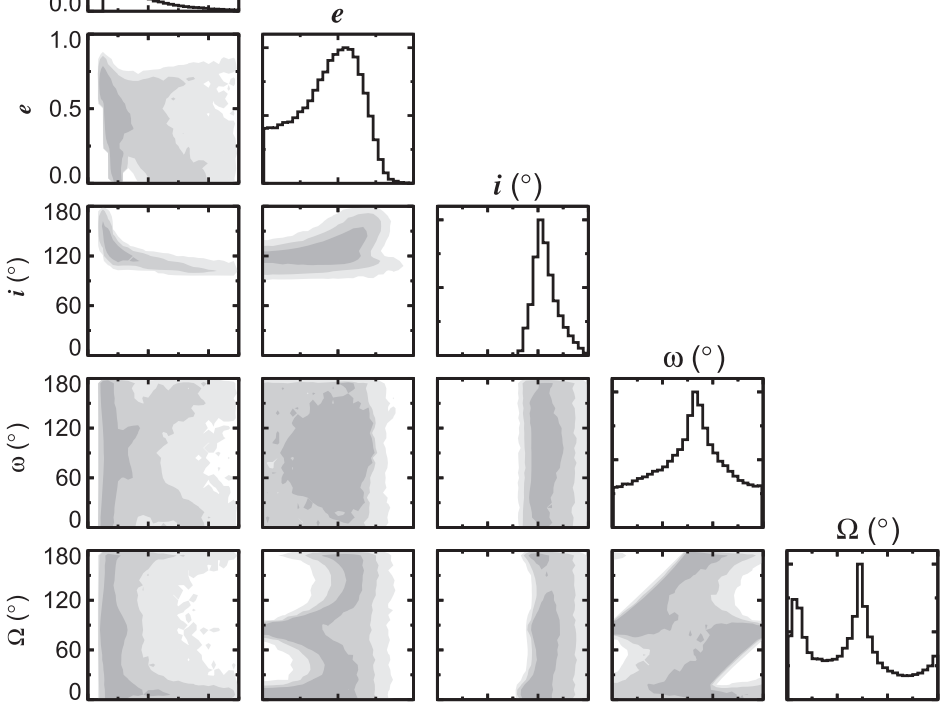

$\tau(\mathrm{yr})$
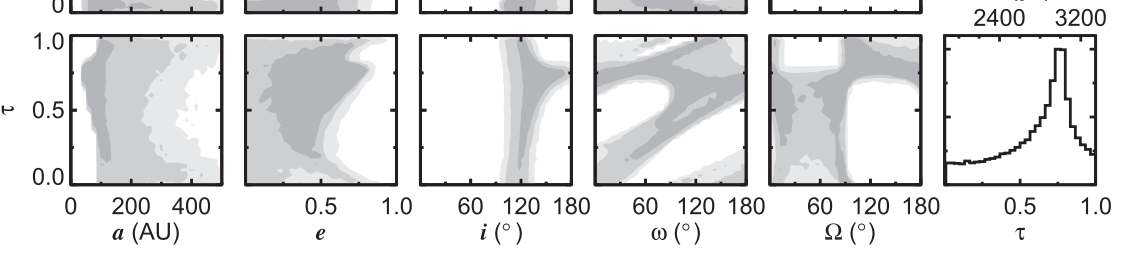

Figure 38. Corner plot for 1 RXS2351+3127 B. See Figure 26 for details. 


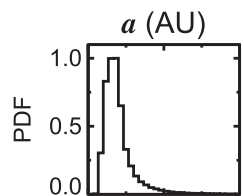

HD 984 B
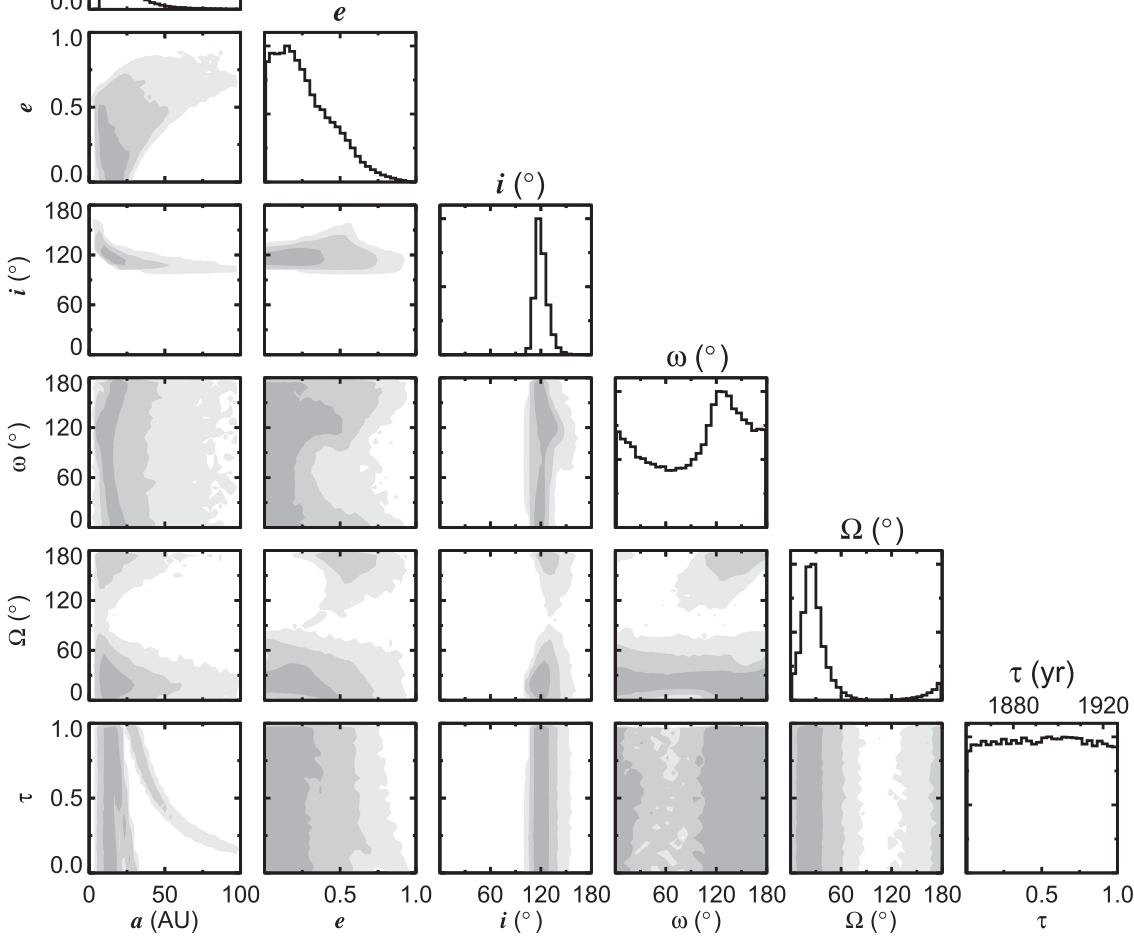

Figure 39. Corner plot for HD 984 B. See Figure 26 for details.

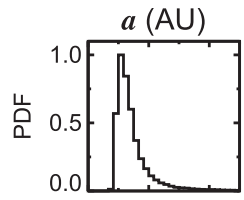

\section{Eri b}
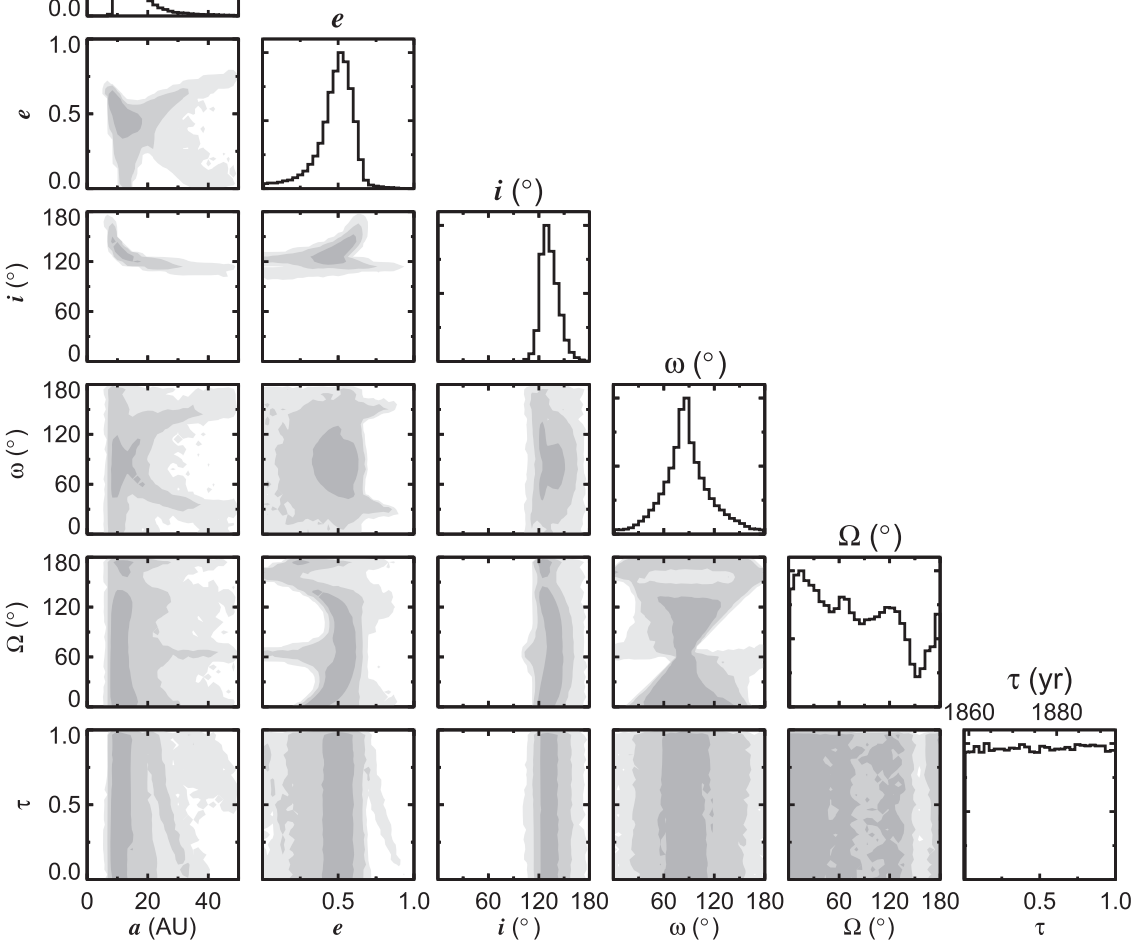

Figure 40. Corner plot for 51 Eri b. See Figure 26 for details. 


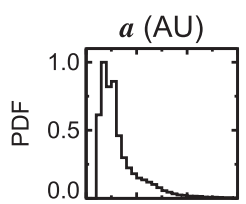

HR 2562 B
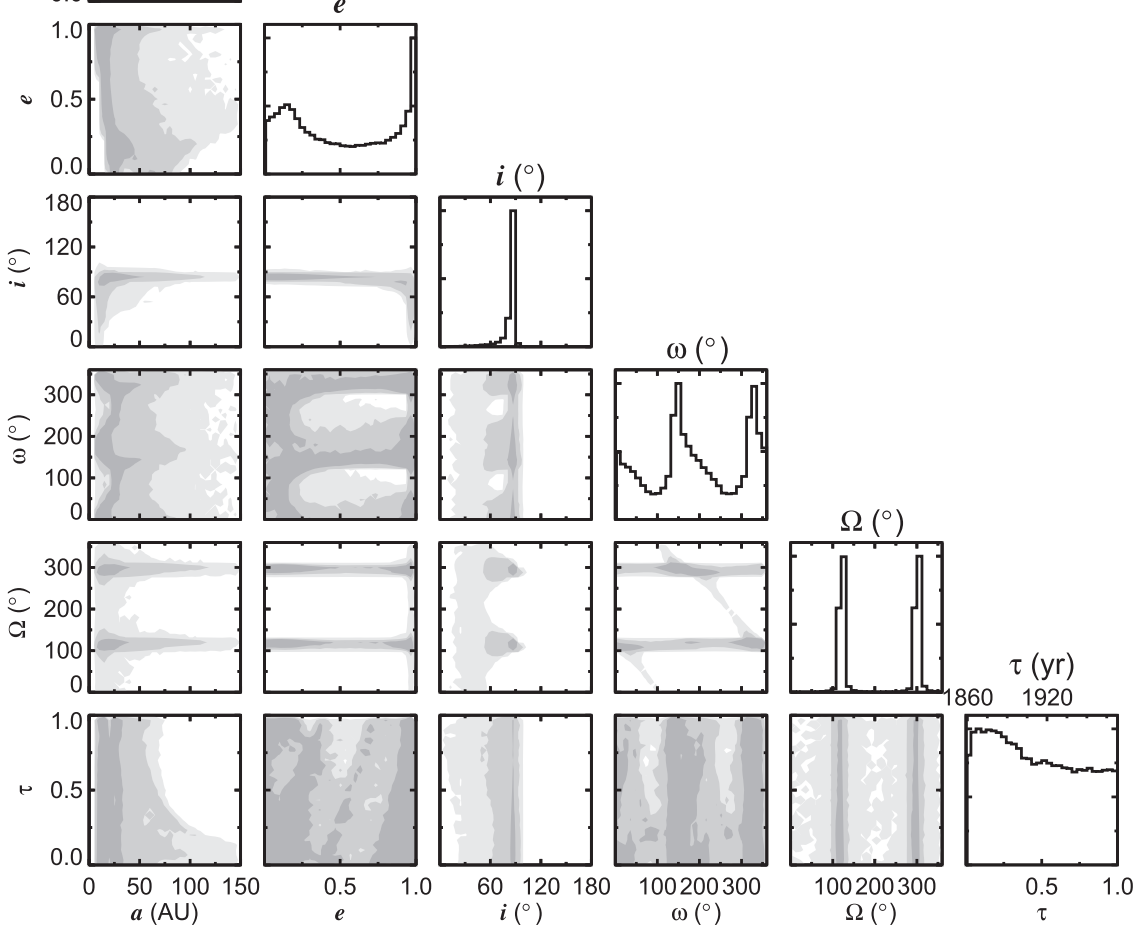

Figure 41. Corner plot for HR 2562 B. See Figure 26 for details.

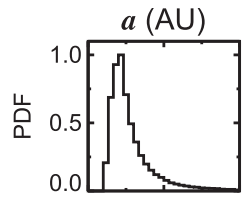

HR 3549 B
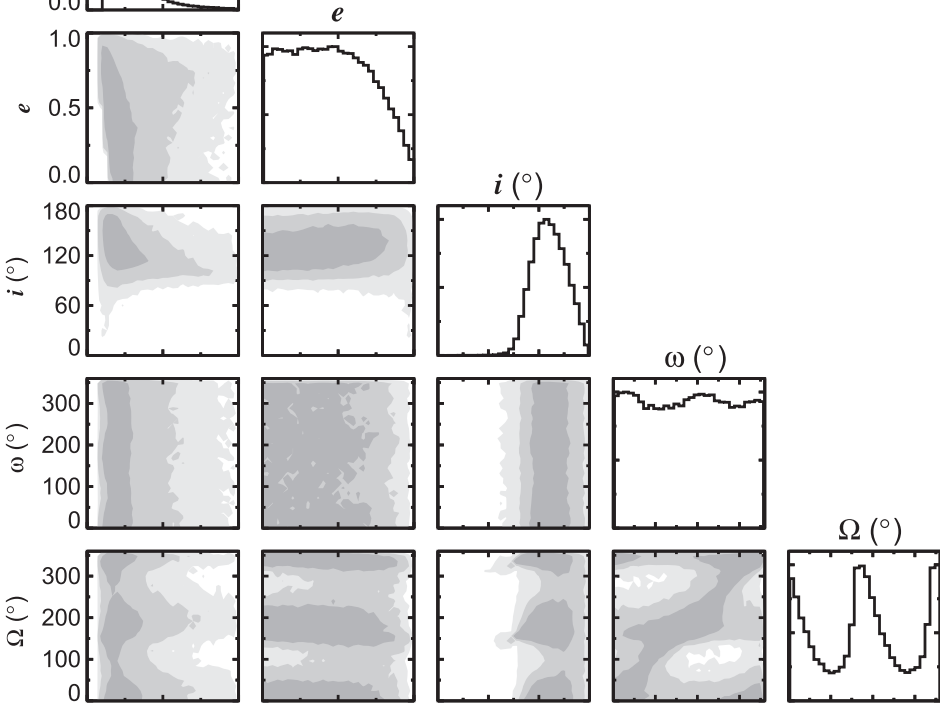

$\tau$ (yr)
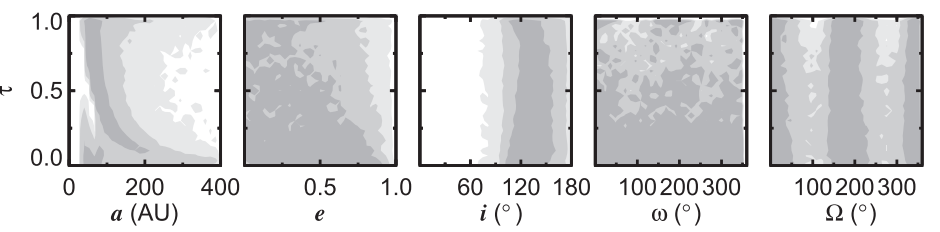

192021602400

Figure 42. Corner plot for HR 3549 B. See Figure 26 for details.

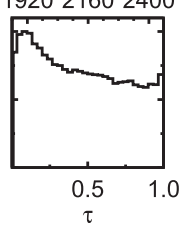




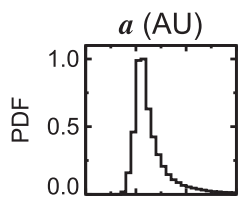

\section{HD 95086 b}
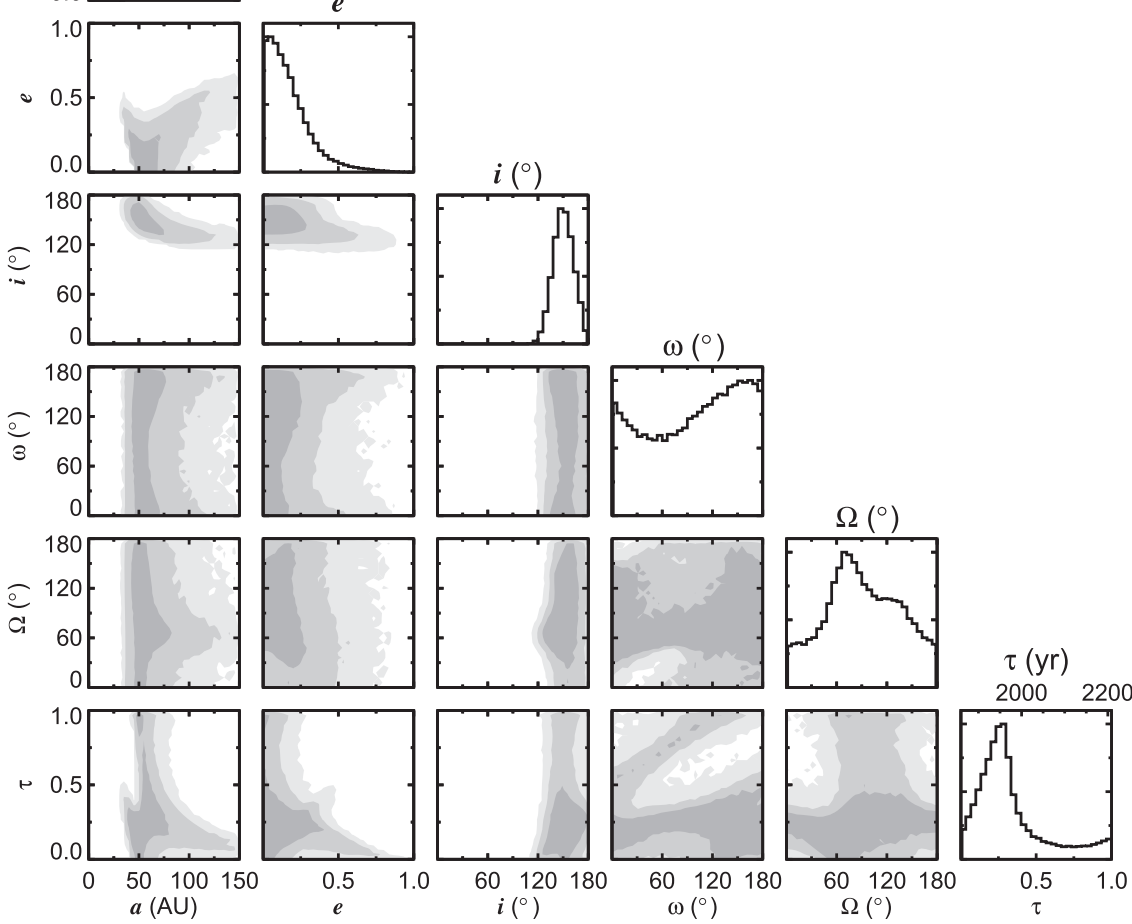

Figure 43. Corner plot for HD 95086 b. See Figure 26 for details.

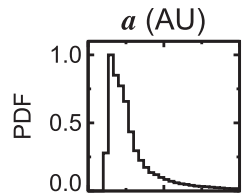

\section{HIP 65426 b}
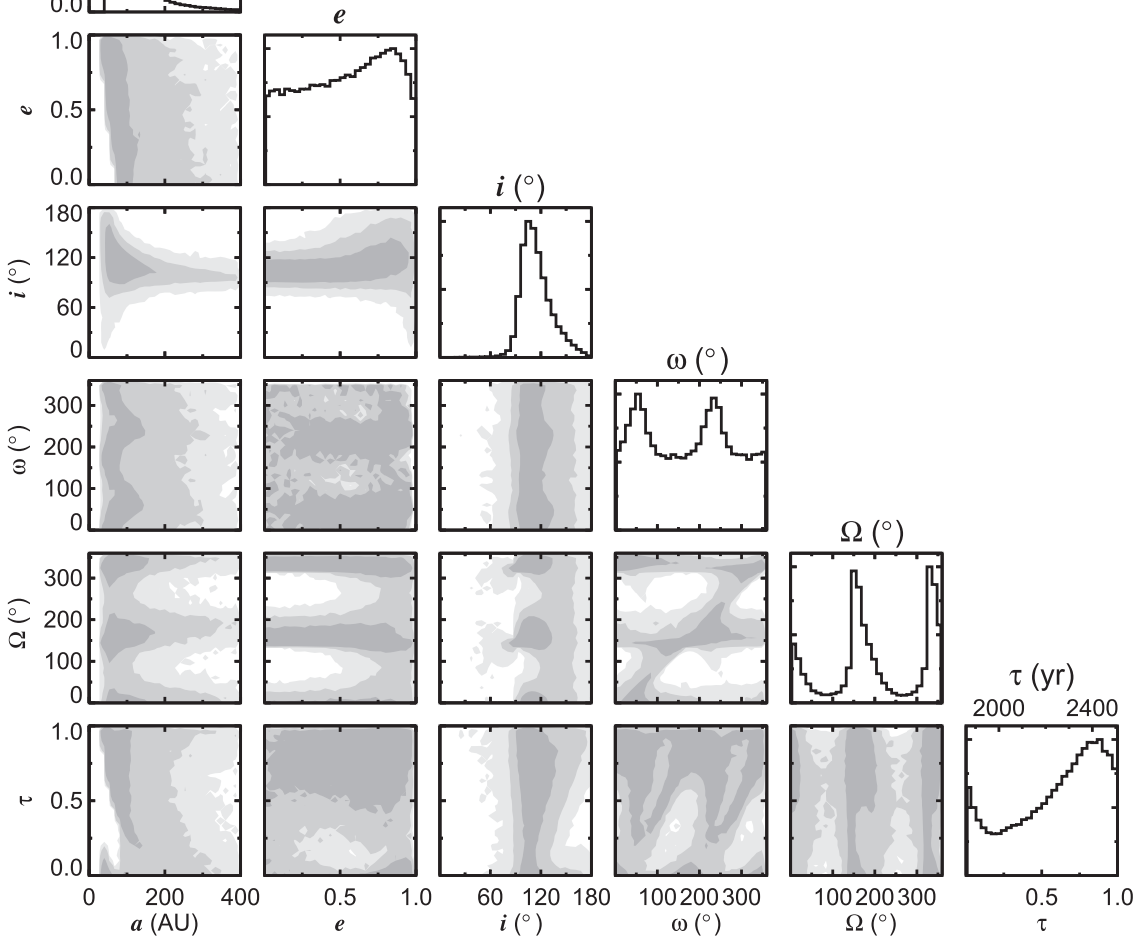

Figure 44. Corner plot for HIP 65426 b. See Figure 26 for details. 


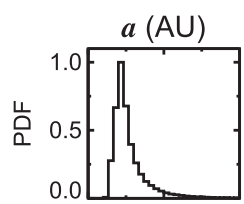

\section{PDS $70 \mathrm{~b}$}
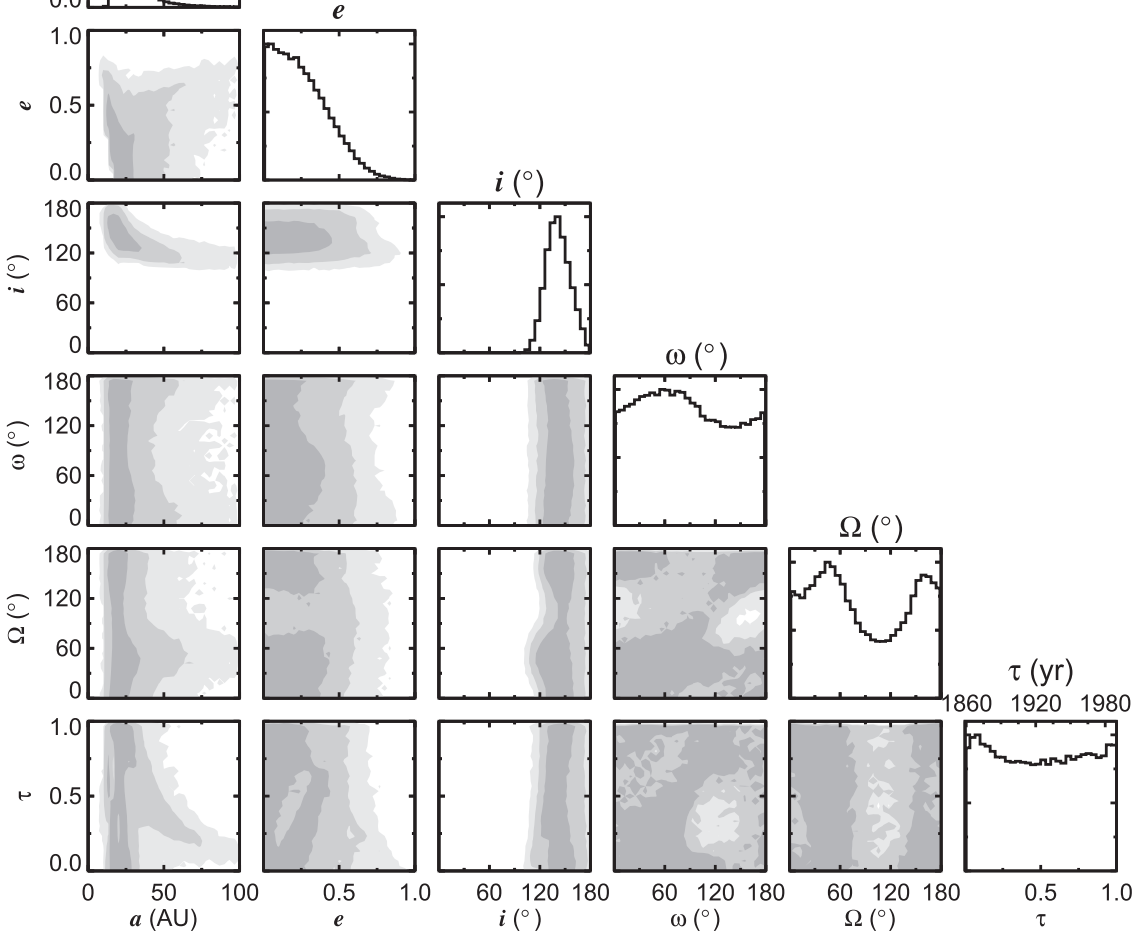

Figure 45. Corner plot for PDS 70 b. See Figure 26 for details.

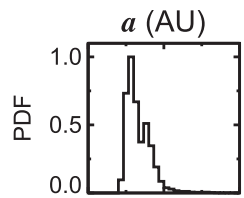

PZ Tel B
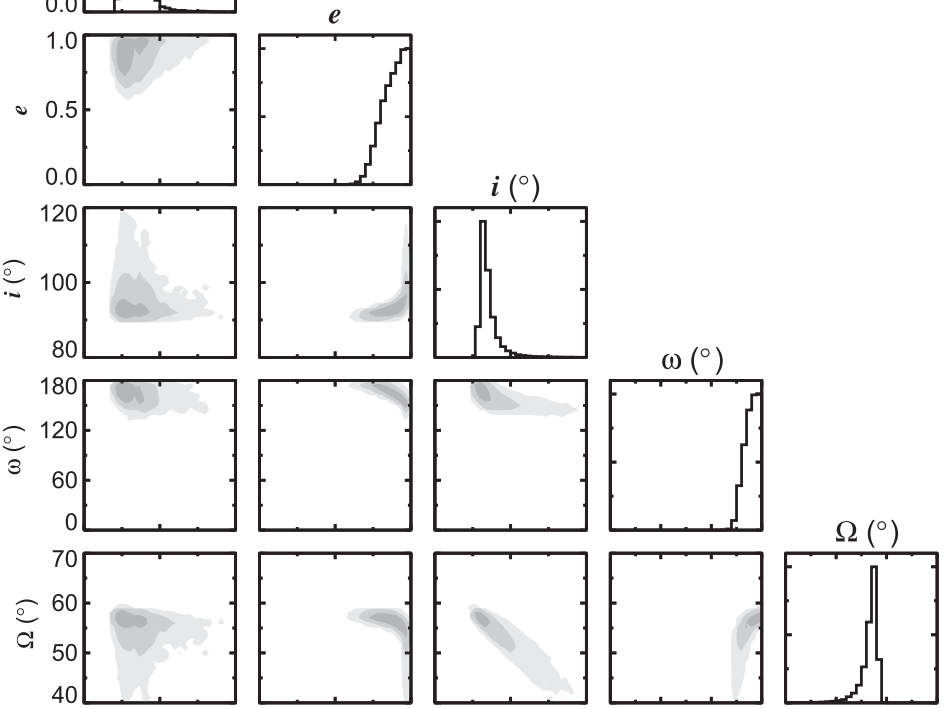

$\tau(\mathrm{yr})$
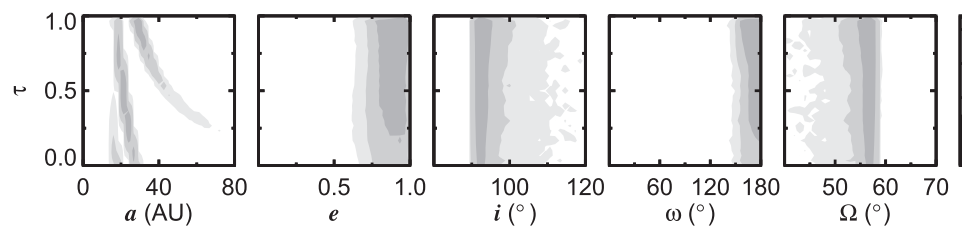

188019201960

Figure 46. Corner plot for PZ Tel B. See Figure 26 for details. 


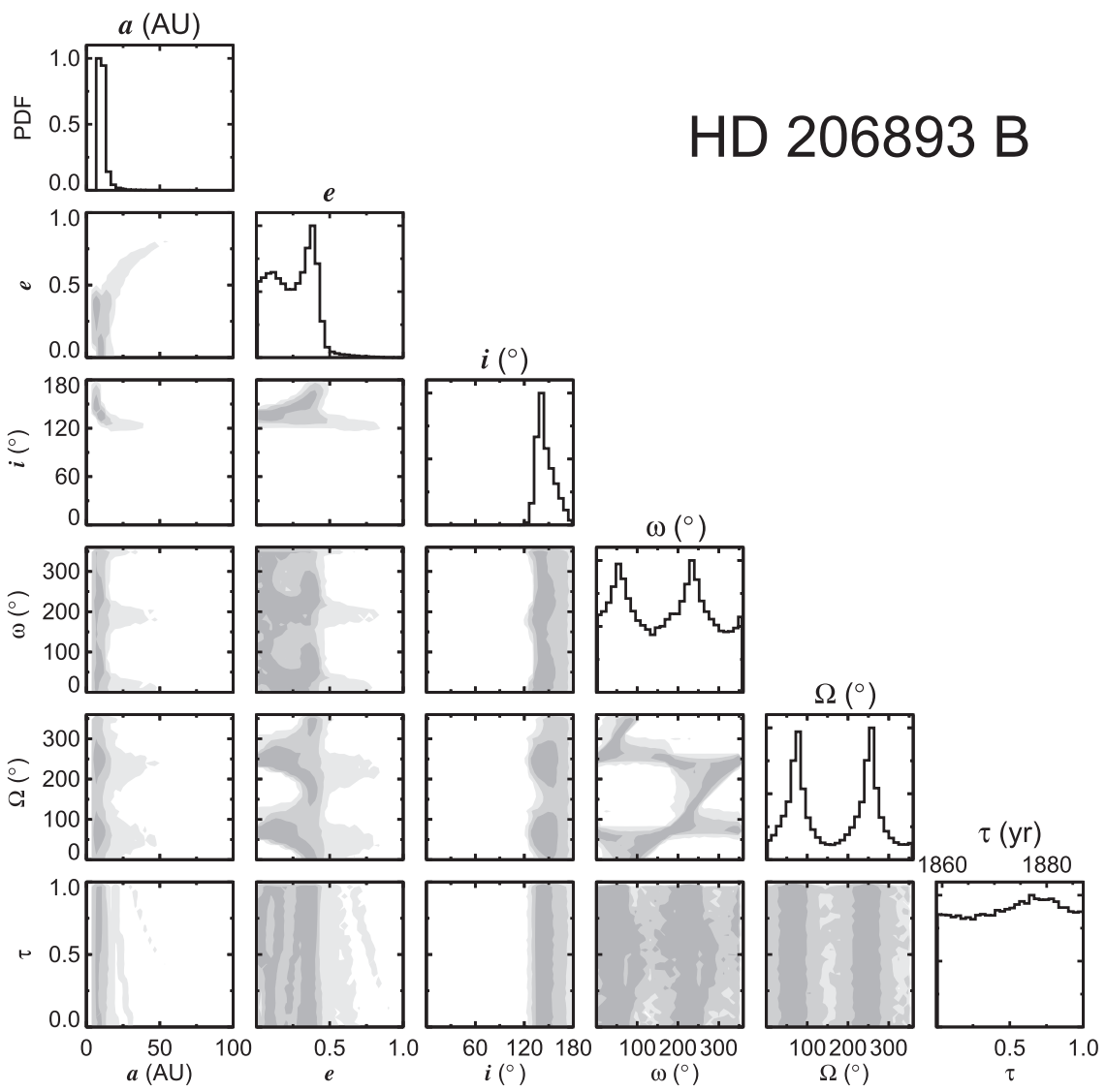

Figure 47. Corner plot for HD 206893 B. See Figure 26 for details.

\section{ORCID iDs}

Brendan P. Bowler (ib https://orcid.org/0000-0003-2649-2288

Sarah C. Blunt (i) https://orcid.org/0000-0002-3199-2888

Eric L. Nielsen (10 https://orcid.org/0000-0001-6975-9056

\section{References}

Alibert, Y., Mordasini, C., Benz, W., \& Winisdoerffer, C. 2005, A\&A, 434, 343

Allen, P. R., Burgasser, A. J., Faherty, J. K., \& Kirkpatrick, J. D. 2012, AJ, 144,62

Ambartsumian, V. A. 1937, AZh, 14, 207

Andrews, S. M., Rosenfeld, K. A., Kraus, A. L., \& Wilner, D. J. 2013, ApJ, 771,129

Bailey, N., \& Fabrycky, D. 2019, AJ, 158, 94

Baraffe, I., Homeier, D., Allard, F., \& Chabrier, G. 2015, A\&A, 577, A42

Baranec, C., Riddle, R., Law, N. M., et al. 2014, ApJL, 790, L8

Barman, T. S., Konopacky, Q. M., Macintosh, B., \& Marois, C. 2015, ApJ, 804,61

Bate, M. R. 2009, MNRAS, 392, 590

Bate, M. R. 2012, MNRAS, 419, 3115

Bate, M. R., Bonnell, I. A., \& Bromm, V. 2002, MNRAS, 332, L65

Best, W. M. J., Liu, M. C., Dupuy, T. J., \& Magnier, E. A. 2017, ApJL, 843, L4

Beust, H., Bonnefoy, M., Maire, A. L., et al. 2016, A\&A, 587, A89 Beuzit, J.-L., Ségransan, D., Forveille, T., et al. 2004, A\&A, 425, 997 Beuzit, J.-L., Vigan, A., Mouillet, D., et al. 2019, A\&A, 631, A155 Biller, B. A., Liu, M. C., Wahhaj, Z., et al. 2010, ApJL, 720, L82 Blunt, S., Nielsen, E. L., De Rosa, R. J., et al. 2017, AJ, 153, 229 Blunt, S., Wang, J., Angelo, I., et al. 2019, arXiv:1910.01756v1 Boley, A. C. 2009, ApJL, 695, L53

Bonnefoy, M., Chauvin, G., Lagrange, A.-M., et al. 2014a, A\&A, 562, A127 Bonnefoy, M., Currie, T., Marleau, G. D., et al. 2014b, A\&A, 562, A111

Bonnefoy, M., Perraut, K., Lagrange, A.-M., et al. 2018, A\&A, 618, A63 Bonnefoy, M., Zurlo, A., Baudino, J. L., et al. 2016, A\&A, 587, A58
Boss, A. P. 1997, Sci, 276, 1836

Boss, A. P. 2001, ApJL, 551, L167

Boss, A. P. 2006, ApJL, 637, L137

Bottom, M., Wallace, J. K., Bartos, R. D., Shelton, J. C., \& Serabyn, E. 2017, MNRAS, 464, 2937

Bowler, B. P. 2016, PASP, 128, 102001

Bowler, B. P., Dupuy, T. J., Endl, M., et al. 2018, AJ, 155, 159

Bowler, B. P., \& Hillenbrand, L. A. 2015, ApJL, 811, L30

Bowler, B. P., Liu, M. C., Shkolnik, E. L., et al. 2012, ApJ, 753, 142

Bowler, B. P., Liu, M. C., Shkolnik, E. L., \& Tamura, M. 2015a, ApJS, 216, 7

Bowler, B. P., \& Nielsen, E. L. 2018, in Handbook of Exoplanets, ed. H. J. Deeg \& J. A. Belmonte (Cham: Springer), 155

Bowler, B. P., Shkolnik, E. L., Liu, M. C., et al. 2015b, ApJ, 806, 62

Brandeker, A., Jayawardhana, R., \& Najita, J. 2003, AJ, 126, 2009

Brandt, T. D. 2018, ApJS, 239, 31

Brandt, T. D., Dupuy, T. J., \& Bowler, B. P. 2019a, AJ, 158, 140

Brandt, T. D., Dupuy, T. J., Bowler, B. P., et al. 2019b, arXiv:1910.01652v1

Brewer, J. M., Fischer, D. A., Valenti, J. A., \& Piskunov, N. 2016, ApJS, 225,32

Bromley, B. C., \& Kenyon, S. J. 2014, ApJ, 796, 141

Bryan, M. L., Bowler, B. P., Knutson, H. A., et al. 2016a, ApJ, 827, 100

Bryan, M. L., Knutson, H. A., Howard, A. W., et al. 2016b, ApJ, 821, 89

Burgasser, A. J., Geballe, T. R., Leggett, S. K., Kirkpatrick, J. D., \& Golimowski, D. A. 2006, ApJ, 637, 1067

Burgasser, A. J., Simcoe, R. A., Bochanski, J. J., et al. 2010, ApJ, 725, 1405 Campante, T. L., Lund, M. N., Kuszlewicz, J. S., et al. 2016, ApJ, 819, 85

Carson, J., Thalmann, C., Janson, M., et al. 2013, ApJL, 763, L32

Chabrier, G., Johansen, A., Janson, M., \& Rafikov, R. 2014, in Protostars and Planets VI, ed. H. Beuther et al. (Tucson: Univ. Arizona Press), 619

Chatterjee, S., Ford, E. B., Matsumura, S., \& Rasio, F. A. 2008, ApJ, 686, 580 Chauvin, G. 2018, Proc. SPIE, 10703, 1070305

Chauvin, G., Desidera, S., Lagrange, A.-M., et al. 2017, A\&A, 605, L9

Chauvin, G., Gratton, R., Bonnefoy, M., et al. 2018, A\&A, 617, A76

Chauvin, G., Lagrange, A.-M., Dumas, C., et al. 2004, A\&A, 425, L29

Chauvin, G., Vigan, A., Bonnefoy, M., et al. 2015, A\&A, 573, A127

Cheetham, A., Ségransan, D., Peretti, S., et al. 2018, A\&A, 614, A16

Cheetham, A. C., Samland, M., Brems, S. S., et al. 2019, A\&A, 622, A80 
Chen, C. H., Mittal, T., Kuchner, M., et al. 2014, ApJS, 211, 25 Chilcote, J., Pueyo, L., De Rosa, R. J., et al. 2017, AJ, 153, 182 Christiaens, V., Cantalloube, F., Casassus, S., et al. 2019, ApJL, 877, L33 Cook, J. D. 2003, Numerical Computation of Stochastic Inequality Probabilities, Tech. Rep. 46, UT MD Anderson Cancer Center Department of Biostatistics

Crepp, J. R., Gonzales, E. J., Bechter, E. B., et al. 2016, ApJ, 831, 136

Crepp, J. R., Johnson, J. A., Fischer, D. A., et al. 2012, ApJ, 751, 97

Crepp, J. R., Johnson, J. A., Howard, A. W., et al. 2014, ApJ, 781, 29

Crepp, J. R., Principe, D. A., Wolff, S., et al. 2018, ApJ, 853, 192

Crepp, J. R., Rice, E. L., Veicht, A., et al. 2015, ApJL, 798, L43

Currie, T., Brandt, T. D., Uyama, T., et al. 2018, AJ, 156, 291

Currie, T., Cloutier, R., Brittain, S., et al. 2015, ApJL, 814, L27

Currie, T., Marois, C., Cieza, L., et al. 2019, ApJL, 877, L3

Curtis, J. L., Agüeros, M. A., Mamajek, E. E., Wright, J. T., \& Cummings, J. D. 2019, AJ, 158, 77

Dawson, R. I., \& Murray-Clay, R. A. 2013, ApJL, 767, L24

De Rosa, R. J., Nielsen, E. L., Blunt, S. C., et al. 2015, ApJL, 814, L3

De Rosa, R. J., Patience, J., Ward-Duong, K., et al. 2014, MNRAS, 445, 3694

Deacon, N. R., Liu, M. C., Magnier, E. A., et al. 2014, ApJ, 792, 119

Delorme, P., Schmidt, T., Bonnefoy, M., et al. 2017, A\&A, 608, A79

Dodson-Robinson, S. E., Veras, D., Ford, E. B., \& Beichman, C. A. 2009, ApJ, 707, 79

Duchêne, G., \& Kraus, A. 2013, ARA\&A, 51, 269

Duffell, P. C., \& Chiang, E. 2015, ApJ, 812, 94

Dupuy, T. J., Brandt, T. D., Kratter, K. M., \& Bowler, B. P. 2019, ApJL, 871, L4

Dupuy, T. J., \& Liu, M. C. 2011, ApJ, 733, 122

Durisen, R. H., Boss, A. P., Mayer, L., et al. 2007, in Protostars and Planets V, ed. B. Reipurth, D. Jewitt, \& K. Keil (Tucson, AZ: Univ. Arizona Press), 607

Fabrycky, D., \& Tremaine, S. 2007, ApJ, 669, 1298

Faherty, J. K., Burgasser, A. J., West, A. A., et al. 2010, AJ, 139, 176

Feigelson, E. D., Lawson, W. A., Stark, M., Townsley, L., \& Garmire, G. P. 2006, AJ, 131, 1730

Ford, E. B., \& Rasio, F. A. 2008, ApJ, 686, 621

Foreman-Mackey, D., Hogg, D. W., Lang, D., \& Goodman, J. 2013, PASP, 125,306

Foreman-Mackey, D., Hogg, D. W., \& Morton, T. D. 2014, ApJ, 795, 64

Forgan, D., Parker, R. J., \& Rice, K. 2014, MNRAS, 447, 836

Fortney, J. J., Marley, M. S., Saumon, D., \& Lodders, K. 2008, ApJ, 683, 1104

Gagné, J., Fontaine, G., Simon, A., \& Faherty, J. K. 2018, ApJL, 861, L13

Galicher, R., Marois, C., Macintosh, B., et al. 2016, A\&A, 594, A63

Gaia Collaboration, Brown, A. G. A., Vallenari, A., et al. 2018, A\&A, 616, 1

Garcia, E. V., Currie, T., Guyon, O., et al. 2017, ApJ, 834, 162

Geller, A. M., Leigh, N. W. C., Giersz, M., Kremer, K., \& Rasio, F. A. 2019, ApJ, 872, 165

Gelman, A., \& Rubin, D. 1992, StaSc, 7, 457

Ginski, C., Schmidt, T. O. B., Mugrauer, M., et al. 2014, MNRAS, 444, 2280

Goldman, B., Marsat, S., Henning, T., Clemens, C., \& Greiner, J. 2010, MNRAS, 405, 1140

Goldreich, P., \& Sari, R. 2003, ApJ, 585, 1024

Grandjean, A., Lagrange, A.-M., Beust, H., et al. 2019, A\&A, 627, L9

Haffert, S. Y., Bohn, A. J., Boer, J., et al. 2019, NatAs, 3, 749

Hastings, W. 1970, Biometrika, 57, 97

Hayano, Y., Takami, H., Oya, S., et al. 2010, Proc. SPIE, 7736, 21

Heintz, W. D. 1990, AJ, 99, 420

Hinkley, S., Pueyo, L., Faherty, J. K., et al. 2013, ApJ, 779, 153

Hodapp, K. W., Suzuki, R., Tamura, M., et al. 2008, Proc. SPIE, 7014, 701419

Hogg, D. W., Myers, A. D., \& Bovy, J. 2010, ApJ, 725, 2166

Huber, D., Bryson, S. T., Haas, M. R., et al. 2016, ApJS, 224, 2

Ireland, M. J., \& Kraus, A. L. 2014, in IAU Symp., Exploring the Formation and Evolution of Planetary Systems, ed. M. Booth, B. C. Matthews, \&

J. R. Graham (Cambridge: Cambridge Univ. Press), 199

Itoh, Y., Hayashi, M., Tamura, M., et al. 2005, ApJ, 620, 984

Janson, M., Bergfors, C., Brandner, W., et al. 2014, ApJS, 214, 17

Janson, M., Hormuth, F., Bergfors, C., et al. 2012, ApJ, 754, 44

Johansen, A., \& Lambrechts, M. 2017, AREPS, 45, 359

Johnson-Groh, M., Marois, C., De Rosa, R. J., et al. 2017, AJ, 153, 190

Jones, J., White, R. J., Quinn, S., et al. 2016, ApJL, 822, L1

Jovanovic, N., Guyon, O., Martinache, F., et al. 2015, PASP, 127, 890

Jurić, M., \& Tremaine, S. 2008, ApJ, 686, 603

Kane, S. R. 2013, ApJ, 766, 10

Keppler, M., Benisty, M., Müller, A., et al. 2018, A\&A, 617, A44

Kervella, P., Arenou, F., Mignard, F., \& Thévenin, F. 2019, A\&A, 623, A72

Kipping, D. M. 2013, MNRAS, 434, L51
Köhler, R., Ratzka, T., Petr-Gotzens, M. G., \& Correia, S. 2013, A\&A, 558, A80

Konopacky, Q. M., Ghez, A. M., Duchêne, G., McCabe, C., \& Macintosh, B. A. 2007, AJ, 133, 2008

Konopacky, Q. M., Rameau, J., Duchene, G., et al. 2016, ApJL, 829, L4

Kratter, K. M., Murray-Clay, R. A., \& Youdin, A. N. 2010, ApJ, 710, 1375

Kraus, A. L., \& Hillenbrand, L. A. 2009, ApJ, 703, 1511

Kraus, A. L., \& Ireland, M. J. 2012, ApJ, 745, 5

Kuzuhara, M., Tamura, M., Kudo, T., et al. 2013, ApJ, 774, 11

Lafrenière, D., Marois, C., Doyon, R., Nadeau, D., \& Artigau, É. 2007, ApJ, 660,770

Lagrange, A.-M., Bonnefoy, M., Chauvin, G., et al. 2010, Sci, 329, 57

Lagrange, A.-M., Meunier, N., Rubini, P., et al. 2019, NatAs, 3, 1135

Lambrechts, M., \& Johansen, A. 2012, A\&A, 544, A32

Lambrechts, M., \& Johansen, A. 2014, A\&A, 572, A107

Limbach, M. A., \& Turner, E. L. 2015, PNAS, 112, 20

Liu, M. C. 2004, Sci, 305, 1442

Liu, M. C., Fischer, D. A., Graham, J. R., et al. 2002, ApJ, 571, 519

Liu, M. C., Wahhaj, Z., Biller, B. A., et al. 2010, Proc. SPIE, 7736, 77361K

Loredo, T. J. 2013, in Astrostatistical Challenges for the New Astronomy, ed. J. M. Hilbe (New York: Springer), 15

Lowrance, P. J., McCarthy, C., Becklin, E. E., et al. 1999, ApJL, 512, L69

Ma, B., \& Ge, J. 2014, MNRAS, 439, 2781

Macintosh, B., Graham, J. R., Barman, T., et al. 2015, Sci, 350, 64

Macintosh, B., Graham, J. R., Ingraham, P., et al. 2014, PNAS, 111, 12661

Macintosh, B. A., Max, C., Zuckerman, B., et al. 2001, in ASP Conf. Ser. 244,

Young Stars Near Earth: Progress and Prospects, ed. R. Jayawardhana \& T. Greene (San Fransisco, CA: ASP), 309

Maire, A. L., Bonnefoy, M., Ginski, C., et al. 2016, A\&A, 587, A56

Maire, A. L., Rodet, L., Cantalloube, F., et al. 2019, A\&A, 624, A118

Maire, A. L., Rodet, L., Lazzoni, C., et al. 2018, A\&A, 615, A177

Malo, L., Doyon, R., Lafrenière, D., et al. 2013, ApJ, 762, 88

Mamajek, E. E., \& Bell, C. P. M. 2014, MNRAS, 445, 2169

Mann, A. W., Dupuy, T., Kraus, A. L., et al. 2019, ApJ, 871, 63

Marleau, G. D., \& Cumming, A. 2014, MNRAS, 437, 1378

Marley, M. S., Fortney, J. J., Hubickyj, O., Bodenheimer, P., \& Lissauer, J. J. 2007, ApJ, 655, 541

Marois, C., Lafrenière, D., Doyon, R., Macintosh, B., \& Nadeau, D. 2006, ApJ, 641, 556

Marois, C., Macintosh, B., Barman, T., et al. 2008, Sci, 322, 1348

Marois, C., Zuckerman, B., Konopacky, Q. M., Macintosh, B., \& Barman, T. 2010, Natur, 468, 1080

Mawet, D., David, T., Bottom, M., et al. 2015, ApJ, 811, 103

Mesa, D., Baudino, J. L., Charnay, B., et al. 2018, A\&A, 612, A92

Mesa, D., Vigan, A., D’Orazi, V., et al. 2016, A\&A, 593, A119

Meshkat, T., Bailey, V., Rameau, J., et al. 2013, ApJL, 775, L40

Meshkat, T., Bonnefoy, M., Mamajek, E. E., et al. 2015, MNRAS, 453, 2379

Metchev, S. A., \& Hillenbrand, L. A. 2004, ApJ, 617, 1330

Metchev, S. A., \& Hillenbrand, L. A. 2009, ApJS, 181, 62

Metropolis, N., Rosenbluth, A., Rosenbluth, M., Teller, A., \& Teller, E. 1953, JChPh, 21, 1087

Milli, J., Hibon, P., Christiaens, V., et al. 2017, A\&A, 597, L2

Mints, A., \& Hekker, S. 2017, A\&A, 604, A108

Montet, B. T., Bowler, B. P., Shkolnik, E. L., et al. 2015, ApJL, 813, 1

Morbidelli, A. 2018, in Handbook of Exoplanets, ed. H. J. Deeg \& J. A. Belmonte (Cham: Springer), 145

Morton, T. D., \& Winn, J. N. 2014, ApJ, 796, 47

Mugrauer, M., Röll, T., Ginski, C., et al. 2012, MNRAS, 424, 1714

Mugrauer, M., Vogt, N., Neuhäuser, R., \& Schmidt, T. O. B. 2010, A\&A, 523, L1

Muirhead, P. S., Dressing, C. D., Mann, A. W., et al. 2018, AJ, 155, 180

Müller, A., Keppler, M., Henning, T., et al. 2018, A\&A, 617, L2

Mustill, A. J., Davies, M. B., \& Johansen, A. 2017, MNRAS, 468, 3000

Nakajima, T., Oppenheimer, B. R., Kulkarni, S. R., et al. 1995, Natur, 378, 463

Naoz, S. 2016, ARA\&A, 54, 441

Nayakshin, S. 2017, MNRAS, 470, 2387

Neves, V., Bonfils, X., Santos, N. C., et al. 2013, A\&A, 551, A36

Nielsen, E. L., De Rosa, R. J., Macintosh, B., et al. 2019, AJ, 158, 13

Nielsen, E. L., Liu, M. C., Wahhaj, Z., et al. 2012, ApJ, 750, 53

Nilsson, R., Veicht, A., Godfrey, P. A. G., et al. 2017, ApJ, 838, 64

Oberg, K. I., Murray-Clay, R., \& Bergin, E. A. 2011, ApJL, 743, L16

Ogilvie, G. I. 2014, ARA\&A, 52, 171

Oppenheimer, B. R., Kulkarni, S. R., Matthews, K., \& Nakajima, T. 1995, Sci, 270,1478

Pearce, L. A., Kraus, A. L., Dupuy, T. J., et al. 2019, AJ, 157, 71

Pearce, T. D., Wyatt, M. C., \& Kennedy, G. M. 2014, MNRAS, 437, 2686 
Perets, H. B., \& Kouwenhoven, M. B. N. 2012, ApJ, 750, 83

Perryman, M., Hartman, J., Bakos, G. Á., \& Lindegren, L. 2014, ApJ, 797, 14 Petrovich, C., \& Tremaine, S. 2016, ApJ, 829, 132

Pollack, J. B., Hubickyj, O., Bodenheimer, P., et al. 1996, Icar, 124, 62 Quanz, S. P., Amara, A., Meyer, M. R., et al. 2013, ApJL, 766, L1 Raghavan, D., McAlister, H. A., Henry, T. J., et al. 2010, ApJS, 190, 1 Raineri, E., Dabad, M., \& Heath, S. 2014, PLoSO, 9, e97349

Rajan, A., Rameau, J., De Rosa, R. J., et al. 2017, AJ, 154, 10

Rameau, J., Chauvin, G., Lagrange, A.-M., et al. 2013a, ApJL, 779, L26

Rameau, J., Chauvin, G., Lagrange, A.-M., et al. 2013b, ApJL, 772, L15

Rameau, J., Follette, K. B., Pueyo, L., et al. 2017, AJ, 153, 244

Rameau, J., Nielsen, E. L., De Rosa, R. J., et al. 2016, ApJL, 822, L29

Rasio, F. A., \& Ford, E. B. 1996, Sci, 274, 954

Reggiani, M., Meyer, M. R., Chauvin, G., et al. 2016, A\&A, 586, A147

Reipurth, B., \& Mikkola, S. 2015, AJ, 149, 145

Rodriguez, D. R., Marois, C., Zuckerman, B., Macintosh, B., \& Melis, C. 2012, ApJ, 748, 30

Rogers, L. A. 2015, ApJ, 801, 41

Sallum, S., Follette, K. B., Eisner, J. A., et al. 2015, Natur, 527, 342

Scharf, C., \& Menou, K. 2009, ApJL, 693, L113

Schlieder, J. E., Lépine, S., \& Simon, M. 2012, AJ, 144, 109

Schneider, J., Dedieu, C., Le Sidaner, P., Savalle, R., \& Zolotukhin, I. 2011, A\&A, 532, A79

Scholz, R.-D. 2010, A\&A, 515, A92

Serabyn, E., Mawet, D., Bloemhof, E., et al. 2009, ApJ, 696, 40

Service, M., Lu, J. R., Campbell, R., et al. 2016, PASP, 128, 095004

Shabram, M., Demory, B.-O., Cisewski, J., Ford, E. B., \& Rogers, L. 2016, ApJ, 820, 93

Shkolnik, E. L., Anglada-Escudé, G., Liu, M. C., et al. 2012, ApJ, 758, 56

Simon, M., \& Schaefer, G. H. 2011, ApJ, 743, 158
Spiegel, D. S., \& Burrows, A. 2012, ApJ, 745, 174

Stone, J. M., Skemer, A. J., Hinz, P. M., et al. 2018, AJ, 156, 286

Suzuki, R., Kudo, T., Hashimoto, J., et al. 2010, Proc. SPIE, 7735, 773530

Tamura, M. 2016, PJAB, 92, 45

Thalmann, C., Carson, J., Janson, M., et al. 2009, ApJL, 707, L123

Thalmann, C., Janson, M., Garufi, A., et al. 2016, ApJL, 828, L17

Todorov, K., Luhman, K. L., \& Mcleod, K. K. 2010, ApJL, 714, L84

Tokovinin, A., \& Kiyaeva, O. 2015, MNRAS, 456, 2070

Udry, S., \& Santos, N. C. 2007, ARA\&A, 45, 397

Van Eylen, V., Albrecht, S., Huang, X., et al. 2019, AJ, 157, 61

Veras, D., Crepp, J. R., \& Ford, E. B. 2009, ApJ, 696, 1600

Vorobyov, E. I. 2013, A\&A, 552, A129

Vousden, W. D., Farr, W. M., \& Mandel, I. 2015, MNRAS, 455, 1919

Wagner, K., Apai, D., \& Kratter, K. M. 2019, ApJ, 877, 46

Wagner, K., Follete, K. B., Close, L. M., et al. 2018, ApJL, 863, L8

Wahhaj, Z., Liu, M. C., Biller, B. A., et al. 2011, ApJ, 729, 139

Wang, J. J., Graham, J. R., Dawson, R., et al. 2018, AJ, 156, 192

Ward-Duong, K., Patience, J., De Rosa, R. J., et al. 2015, MNRAS, 449, 2618

Webb, R. A., Zuckerman, B., Platais, I., et al. 1999, ApJL, 512, L63

Weidenschilling, S. J., \& Marzari, F. 1996, Natur, 384, 619

Winn, J. N., \& Fabrycky, D. C. 2015, ARA\&A, 53, 409

Wizinowich, P., Acton, D. S., Shelton, C., et al. 2000, PASP, 112, 315

Wolfgang, A., Rogers, L. A., \& Ford, E. B. 2016, ApJ, 825, 19

Wright, J. T., Upadhyay, S., Marcy, G. W., et al. 2009, ApJ, 693, 1084

Wu, Y.-L., Sheehan, P. D., Males, J. R., et al. 2017, ApJ, 836, 223

Xie, J.- W., Dong, S., Zhu, Z., et al. 2016, PNAS, 113, 11431

Yamamoto, K., Matsuo, T., Shibai, H., et al. 2013, PASJ, 65, 90

Yelda, S., Lu, J. R., Ghez, A. M., et al. 2010, ApJ, 725, 331

Zuckerman, B., \& Song, I. 2009, A\&A, 493, 1149 